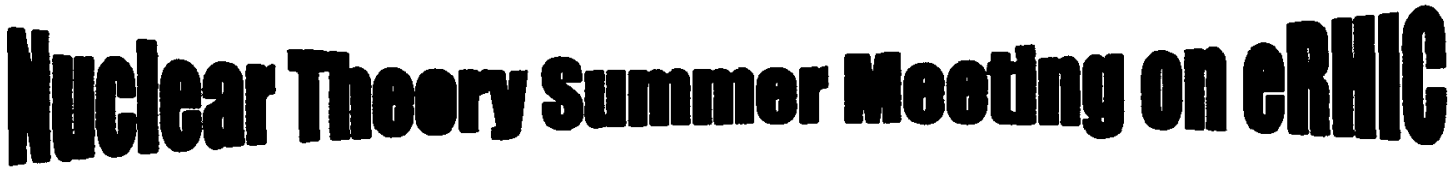

June 26 to July 14, 2000

\title{
Workshop Proceedings
}

November 11, 2000 
BNL-52606

Formal Report

\section{Nuclear Theory Summer Meeting on eRHIC Brookhaven National Laboratory}

June 26 to July 14, 2000

\section{$\underline{\text { Organizers }}$}

L. McLerran and R. Venugopalan

Brookhaven National Laboratory

Brookhaven National Laboratory

Brookhaven Science Associates

Upton, Long Island, New York 11973

Under Contract No. DE-AC02-98CH10886

UNITED STATES DEPARTMENT OF ENERGY 


\title{
DISCLAIMER
}

This report was prepared as an account of work sponsored by an agency of the United States Government. Neither the United States Government nor any agency thereof, nor any of their employees, not any of their contractors, subcontractors, or their employees, makes any warranty, expressed or implied, or assumes any legal liability or responsibility for the accuracy, completeness, or usefulness of any information, apparatus, product or process disclosed, or represents that its use would not infringe privately owned rights. Reference herein to any specific commercial product, process, or service by trade name, trademark, manufacturer, manufacturer, or otherwise, does not necessarily constitute or imply it endorsement, recommendation, or favoring by the United States Government or any agency, contractor, or subcontractor thereof. The views and opinions of authors expressed herein do not necessarily state of reflect those of the United States Government or any agency, contractor or subcontractor thereof.

\author{
Printed in the United States of America \\ Available from \\ National Technical Information Service \\ U.S. Department of Commerce \\ 5285 Port Royal Road \\ Springfield, VA 22161
}




\section{Preface}

The eRHIC BNL summer meeting was held at BNL from June 26 to July 14, 2000. The meeting was very informal with only two talks a day and with ample time for discussions and collaborations. Several of the theory talks focused on the issue of saturation of parton distributions at small $\mathrm{x}$ - whether screening effects have already been seen at HERA, the relation of saturation to shadowing, and on the various signatures of a proposed novel state of matter - the Colored Glass Condensate - that may be observed at eRHIC. A related topic that was addressed was that of quantifying twist four effects, and on the relevance of these for studies of energy loss. Other issues addressed were coherence effects in vector meson production, anti-quark distributions in nuclei, and the relevance of saturation for heavy ion collisions. There were, also, talks on the Pomeron the relevance of instantons and the non-perturbative gluon condensate to constructing a Pomeron.

On the spin physics side, there were talks on predictions for inclusive distributions at small x. There were, also, talks on Skewed Parton Distributions and Deeply Virtual Compton Scattering.

Though most of the talks were theory talks, there were, also, several important experimental contributions. A preliminary detector design for eRHIC was presented. Studies for semi-inclusive measurements at eRHIC were also presented. The current status of pA scattering studies at RHIC was also discussed.

The eRHIC summer meeting provided a vigorous discussion of the current status of eRHIC studies. It is hoped that this document summarizing these discussions will be of use to all those interested in electron nucleus and polarized electron-polarized proton studies.
L. McLerran
R. Venugopalan 


\section{Table of Contents}

High twist contributions to Nuclear Structure Functions

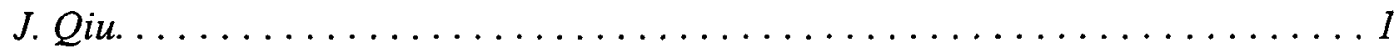

Diffraction and nuclear shadowing

M. Strikman.

Tuesday, June 27, 2000

Small Seminar Room

Spin Physics Possibilities at eRHIC

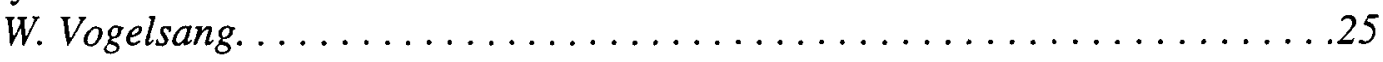

Role of nuclear shadowing and antishadowing in DIS on polarized nuclear targets

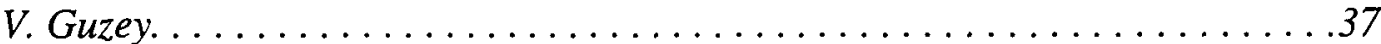

Wednesday, June 28, 2000

Small Seminar Room

A manifestation of gluon saturation in eA DIS -I

E. Levin. . . . . . . . . . . . . . . . . . . . . . . . . . . 47

A manifestation of gluon saturation in eA DIS -II

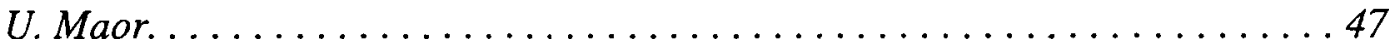

Thursday, June 29, 2000

Small Seminar Room

Recent Work on Renormalization Group for the Colored Glass Condensate

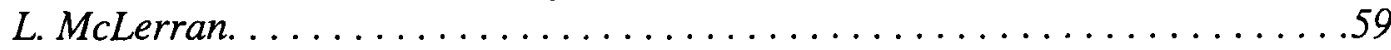

Parton Energy Loss and Modified Fragmentation Functions in Deeply Inelastic eA

Scattering

$X-N$. Wang. . . . . . . . . . . . . . . . 65

Friday, June 30, 2000

Small Seminar Room

$\mathrm{A}^{\propto}$ - Physics at eRHIC

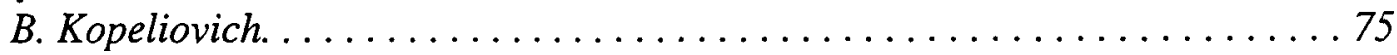

Anti-Quarks in Nucleons and Nuclei-The Role of Virtual Mesons

G. Garvey. . . . . . . . . . . . . . . . . . . . . . . . . .93 
Physics at eRHIC - Selected Topics

W. Krasny.....................................115

Exploring skewed parton distributions with polarized targets

M. Diehl. ...................................123

A QCD analysis of polarized parton densities

D. Indumathi. ......................................

Friday, July 7,2000

Small Seminar Room

Initial Gluon Energy and Multiplicity in Heavy-ion Collisions or Exciting a Colored Glass Condensate

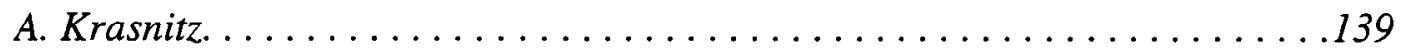

Deeply virtual Compton scattering at small $\mathrm{x}$

I. Balitsky. ................................... 153

Monday, July 10,2000

Small Seminar Room

P-A Physics at RHIC

$J-C$. Peng. .................................. 161

Physics Issues at eRHIC

Y. Kovchegov................................ 173

Tuesday, July 11, 2000

Small Seminar Room

Gluon Shadowing: Perturbative or not?

J. Jalilian-Marian. ............................... 79

Wednesdav, July 12, 2000

Small Seminar Room

Computing the Gluon Structure Functions of Nucleons and Nuclei

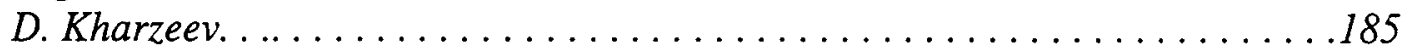

Diffractive Physics at eRHIC

K. Itakura.

Thursday, July 13,2000

Small Seminar Room

Hard Exclusive Processes at eRHIC - Experimental Aspects

A. Sandacz.................................. 193/217 


\title{
High Twist Contributions to Nuclear Structure Functions
}

\author{
Xiaofeng Guo ${ }^{a}$, Jianwei $\mathrm{Qiu}^{b}$ and Wei $\mathrm{Zhu}^{c}$ \\ ${ }^{a}$ Department of Physics and Astronomy, University of Kentucky \\ Lexington, KY 40506, USA \\ ${ }^{b}$ Department of Physics and Astronomy, Iowa State University \\ Ames, Iowa 50011, USA \\ 'Physics Department, China East Normal University \\ Shanghai, P.R. China
}

\begin{abstract}
We investigate the high twist contributions (or power corrections) to the effective nuclear structure functions measured in deeply inelastic lepton-nucleus scattering. For a heavy nuclear target of atomic weight $A$, we show that the $A^{1 / 3}$ enhanced power corrections, $\left(A^{1 / 3} / Q^{2}\right)^{N}$, can be systematically summed to all order in $N$. At leading order in $\alpha_{s}$, the $A^{1 / 3}$ enhanced power corrections, $\left(\alpha_{s} A^{1 / 3} / Q^{2}\right)^{N}$, can be summed into an exponential form when $N \rightarrow \infty$. Our results show a small numerical effect in the shadowing region, and are most relevant to effective nuclear structure functions in the region of old EMC effect.
\end{abstract}

\section{Summary of our results}

It was discovered by European Muon Collaboration (EMC) in early eighties that the nuclear structure functions measured in high energy deeply inelastic scattering (DIS) are very different from a simple sum of individual nucleon structure functions. Many experiments have been carried out since then, and the observed nuclear dependence, known as the EMC effect, have been verified and better measured. Although nuclear binding energy is much smaller than the energy exchange in the collisions, measured nuclear structure functions normalized by the atomic weight can differ from free space nucleon structure functions by as much as 10 or more percents. The ratio of the nuclear and nucleon structure functions, $R_{F}\left(x_{B}, Q^{2}\right)$, has a very nontrivial functional dependence on the Bjorken variable $x_{B}$; which can be either larger or less than one. The full range of $x_{B}$ is often divided into thrce regions: shadowing $\left(x_{B}<0.1\right)$, old EMC $\left(0.1<x_{B}<\right.$ $0.6 \sim 0.7)$, and Fermi motion $\left(x_{B}>0.6 \sim 0.7\right)$. 
If the framework of QCD factorization is valid for deeply inelastic scattering on nuclear target, there are only two possible sources for the observed nuclear dependence: long-distance nuclear matrix elements, and perturbatively calculable partonic parts. When $x_{B}<0.1$, the wave length of the virtual photon in DIS covers more than one nucleon (or the photon can interact with more nucleons inside a large nucleus). It is such coherent interaction that is responsible for the observed nuclear shadowing. Working in the target rest frame, a QCD Glauber formalism was developed to calculate such shadowing effect, and the observed shadowing can be thought as a result of multiple scattering between the hadronic components of the photon and the nucleons. Working in the infinite momentum frame, however, a different physical picture of the shadowing is more relevant: shadowing is a result of coherent interactions between the soft (or wee) partons from different nucleons.

When $x_{B}$ is larger than 0.1 , the probe (the virtual photon) has a wave length smaller than nucleon size, and therefore, we expect that the interactions are localized within individual nucleons inside a large nucleus. Many models, such as pion-cloud model, rescaling model, quark cluster model, and etc., have been developed and used to fit the experimental data in this so-called old EMC region. All these models are concentrated on the changes of nucleon parton distributions once confined inside a large nucleus.

In this talk, we present a QCD calculation which sums up nuclear effects due to final-state interactions, which cover interactions between nuclear matter and the partons struck by the virtual photon. Although such effect are suppressed by the powers of energy scale of the virtual photon $Q^{2}$, it is enhanced by nuclear size $A^{1 / 3}$. For a large nucleus and not too large $Q^{2}$, such nuclear size enhanced effect may be important.

Working in the infinite momentum frame, we systematically calculated the nuclear size enhanced power corrections (or high twist effects) to the effective nuclear structure functions.

- we demonstrated that when the Bjorken variable $x_{B}$ is not too small (not in deep shadowing region), only the final-state multiple scattering between the struck quark and the rest nucleons give the leading power corrections to the measured nuclear structure functions.

- We show that the nuclcar size enhanced power corrections can be summed to all orders in power corrections. At leading order in $\alpha_{s}$, the nuclear size enhanced power corrections, $\left(\alpha_{s} A^{1 / 3} / Q^{2}\right)^{N}$, can be summed into an exponential form when $\lambda \rightarrow \infty$.

- With the cxponcutial form, the net effect of the power corrections to the measured 
nuclear structure functions is equal to a shift in $\log \left(1 / x_{B}\right)$.

- Because of the logarithmic dependence, our results show a small numerical effect in the shadowing region, and are most relevant to effective nuclear structure functions in the region of old EMC effect.

- Combining the Fermi motion effect in the region of large $x_{B}$, our results with resumed power corrections in QCD naturally explain the observed EMC effect.

- Numerical plots will be shown in our full length paper, and a copy of ten transparencies of my talk is attached. 
(

4. Multiple Scattering and Power Corrections.

* For $Q^{2}<1$ GeV, power corrections (o rhigh twist Contributions) become more important!

* Conventional perturbative power series:

$$
\begin{aligned}
& F\left(x_{B}, Q^{2}\right)=\sum_{q}\left(\sum_{m=0}^{\infty} C^{(m)}(x)\left(\frac{\alpha_{s}}{\pi}\right)^{m}\right) \otimes q\left(x \cdot \Delta^{2}\right) \\
& + \text { gluon part }
\end{aligned}
$$

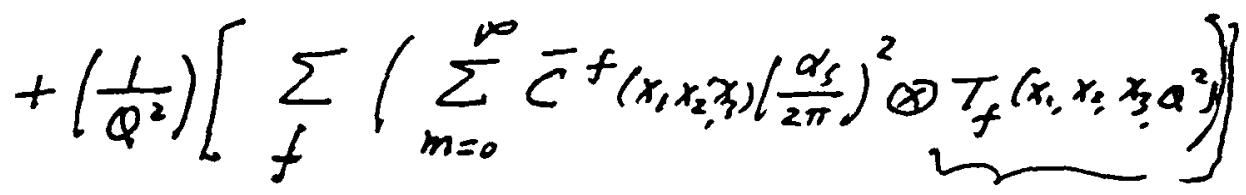

$$
\begin{aligned}
& +\left(\frac{1}{Q^{2}}\right)^{2}[\cdots]+\cdots
\end{aligned}
$$

- How much do we know about the correlation functions? - Nuclear effect is special,

- How many terms of power corrections (on how many scattering) should we include? - depend on nuclear size. 
(4)

* Space-time Picture of DIS:

- Let the nucleus moves along $z$-axis:
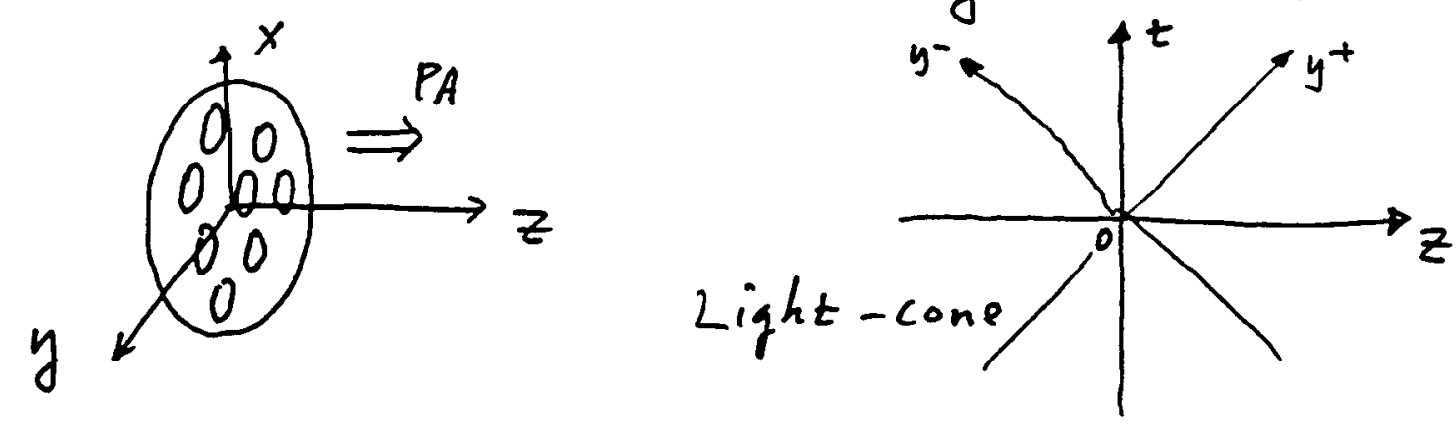

- Nucleons on a transverse plane arrive at $z=0$ at the same time $t$.

- Nucleons along the same impact parameter arrive at $z=0$ at different time $t$.

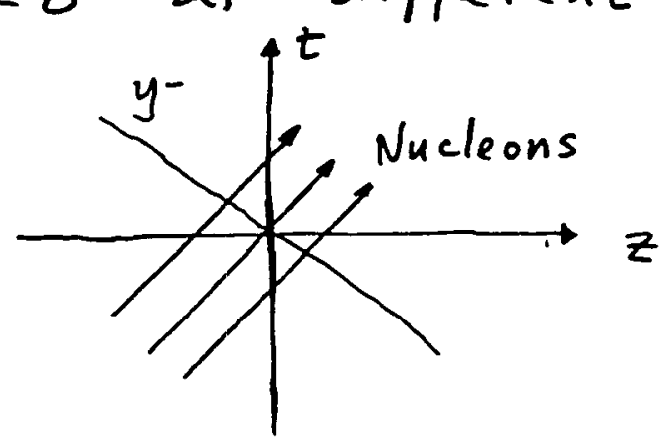

$000) \rightarrow z$

- In DIS, quark moving along " "direction moves along "-" direction, after absorb the virtual photon:
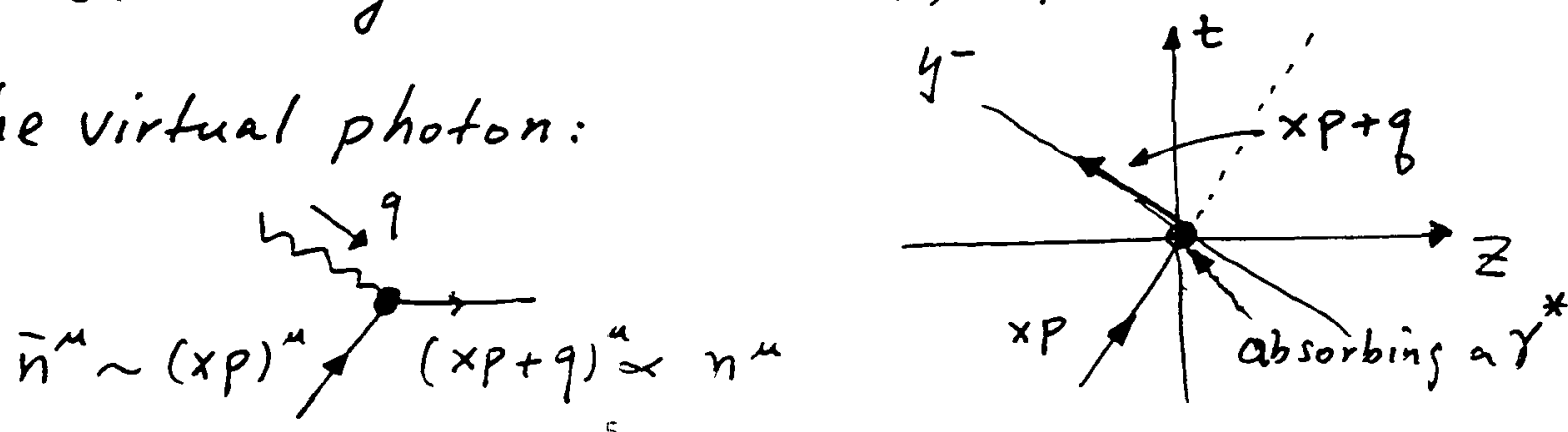
(10)

- The scattered quark can interact with Parton from nucleons along the same impact parameter:
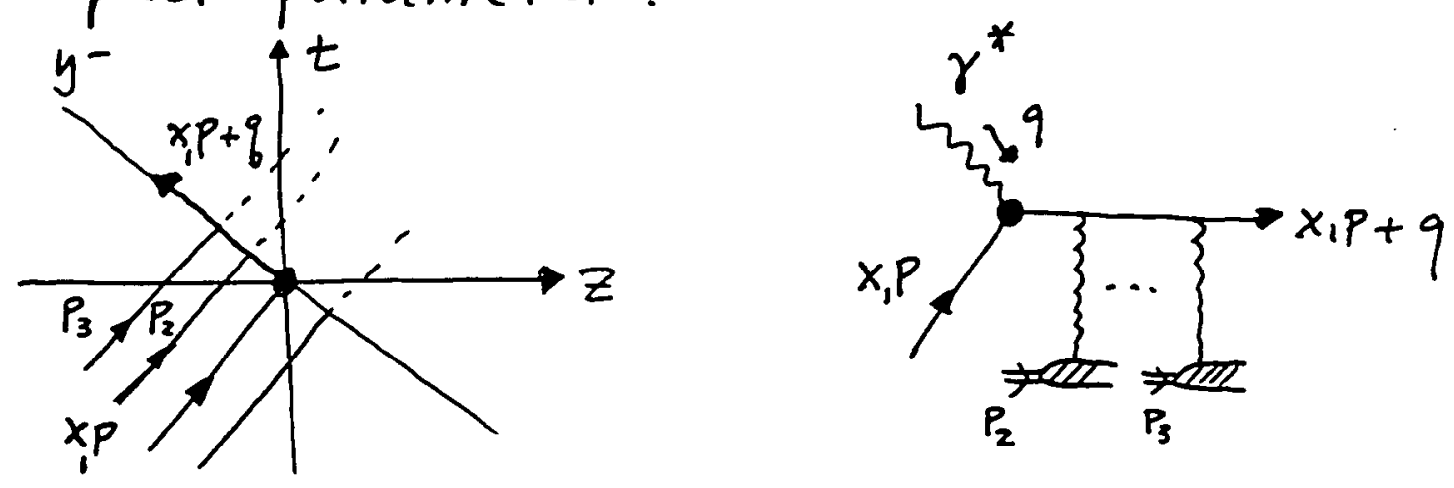

- Only interactions at the same $y^{+}$contribute. Example:

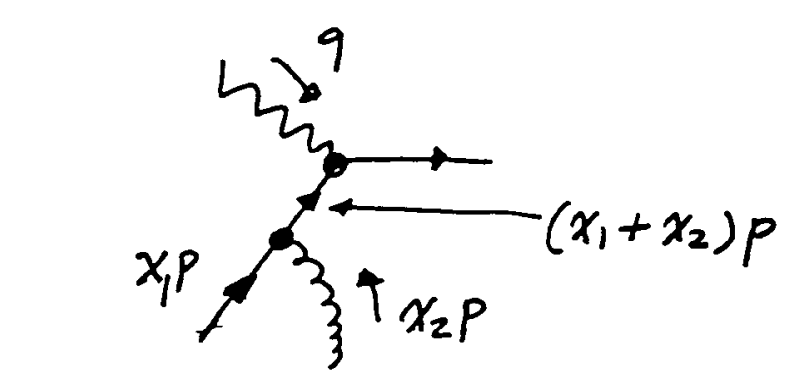
only the special' propagator piece contribute to the perturbative part.

$$
\begin{aligned}
& \stackrel{k}{\longrightarrow}=\frac{r \cdot \hat{k}}{k^{2}}+\frac{\gamma \cdot n}{2 k \cdot n} \text { - special propagator } \\
& \text { [Notation to }
\end{aligned}
$$

- Multiple Scattering is suppressed by $\frac{1}{a^{2}}$, but, it's enhanced by $A^{1 / 3}$ (one direction). 
5. Resume $\left(\frac{\alpha_{s} A^{1 / 3}}{Q^{2}}\right)^{m}$ contributions

(11)

* Subprocesses:

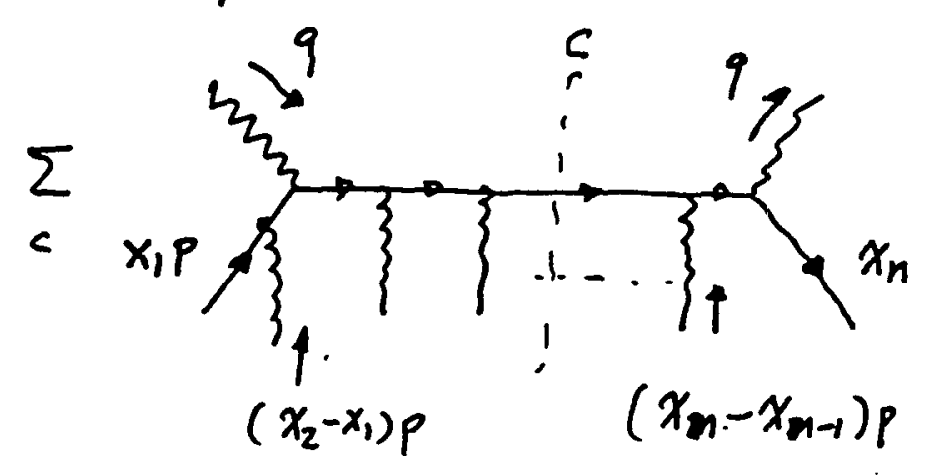

Leading diagrams in infinite momentum frame.

* Nuclear wave functions:

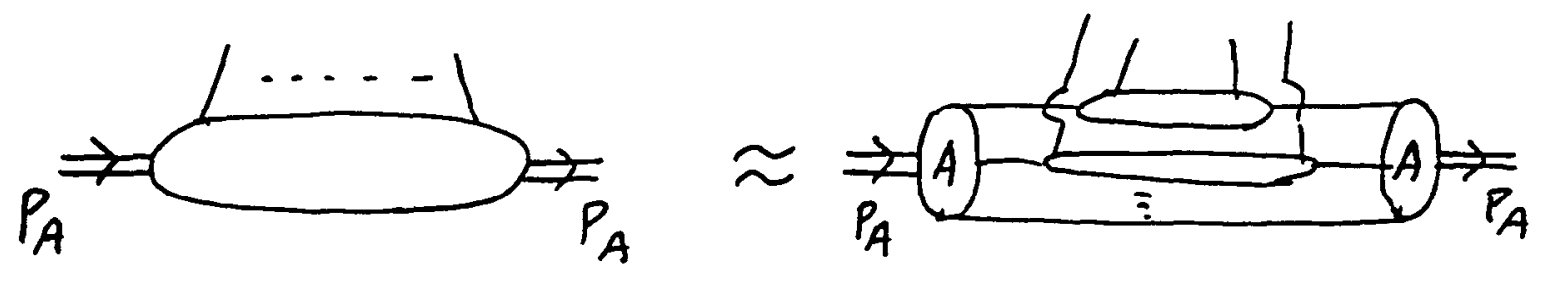

- Partons are paired,

- One pair for one nucleon

- nucleons are color singlet.

- Normalization:

$$
1=\int d^{3} p|p\rangle\langle p| \quad\left\langle p \mid p^{\prime}\right\rangle=\delta^{3}\left(p-p^{\prime}\right)
$$

- Nuclear wave function:

$$
\left\langle p_{A} \mid p_{1}, p_{2}, \ldots p_{A}\right\rangle \equiv \psi\left(p_{A}, p_{1}, p_{2} \ldots p_{A}\right) \delta^{(3)}\left(p_{A}-\sum_{i=1}^{A} p_{i}\right)
$$

7 
(12)

* Nuclear density matrices:

- Matrix: $T_{A} \equiv\left\langle P_{A}\left|\hat{O}\left(y_{1}, y_{2} \ldots\right)\right| P_{A}\right\rangle$

- The $\hat{O}$ involves only one nucleon: $=$

$$
\begin{aligned}
T_{A} & =\int d^{3} p_{1} d^{3} p_{1}^{\prime} \rho\left(p_{1}, p_{1}^{\prime}\right)\left\langle p_{1}\left|\hat{o}\left(y_{1}, y_{2} y_{2} \ldots\right)\right| p_{1}^{\prime}\right\rangle \\
& =\int d^{3} p_{1} \rho\left(p_{1}\right)\left\langle p_{1}\left|\hat{o}\left(y_{1}, y_{2} \ldots\right)\right| p_{1}\right\rangle
\end{aligned}
$$

density matin:

$$
\rho\left(p_{1}\right)=\int\left(\prod_{i=2}^{A} d^{3} p_{i}\right)\left|\psi\left(p_{A_{j}} p_{1}, p_{2} \ldots\right)\right|^{2} \delta^{(3)}\left(p_{1}-\left(p_{A}-\sum_{i=2}^{A} p_{i}\right)\right)
$$

- The $\hat{O}$ involves two nucleons:

$$
\begin{aligned}
& T_{A}=\int d^{3} P_{1} d^{3} P_{2} d^{3} P_{1}^{\prime} d^{3} P_{2}^{\prime} P\left(P_{1}, P_{2}, P_{1}^{\prime}, P_{2}^{\prime}\right) \\
& +\left\langle p_{1}, p_{2}\left|\hat{O}\left(y_{1}, y_{2} \ldots\right)\right| p_{1}^{\prime}, p_{2}^{\prime}\right\rangle \\
& =\int d^{3} p_{1} d^{3} p_{2} d^{3} \delta \rho\left(p_{1}, p_{2}, p_{1}-\delta, p_{2}+\delta\right) \\
& *\left\langle p_{1}, p_{2}\left|\hat{O}\left(y_{1}, y_{2} \ldots\right)\right| p_{1}-\delta, p_{2}+\delta\right\rangle \\
& P\left(P_{1}, P_{2}, P_{1}-\delta, P_{2}+\delta\right)=\int\left(\prod_{i=3}^{A} d^{3} P_{i}\right) \psi\left(P_{A} ; P_{1}, P_{2} \cdots P_{A}\right) \psi\left(P_{A} ; P_{1}-\delta, P_{2}+\delta, P_{3} \cdots P_{A}\right) \\
& +\delta^{3}\left(P_{A}-\sum_{i=1}^{4} p_{i}\right) \\
& \vdots
\end{aligned}
$$

8 
(15)

* Leading contribution in $\left(\frac{\alpha_{S} A^{1 / 3}}{Q^{2}}\right)^{m}$,

- Diagrams:
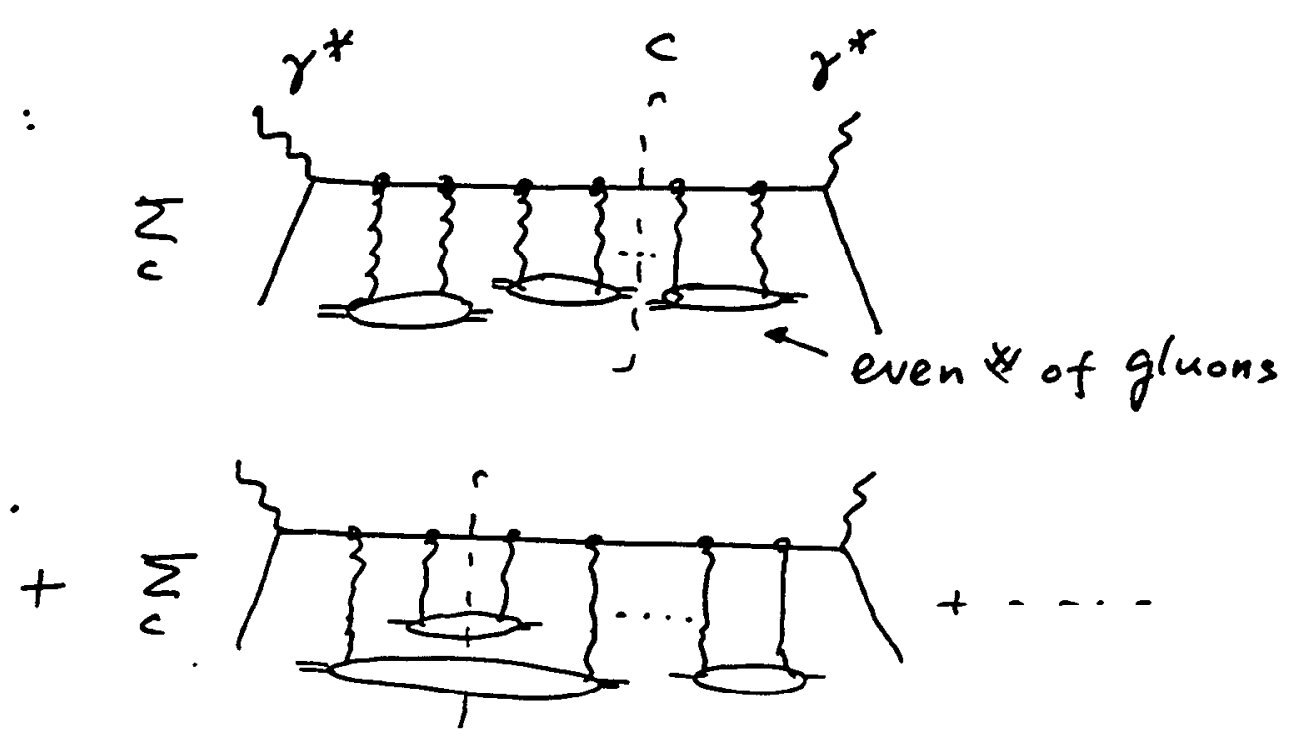

-Three types "effective quark-gluon vertices":

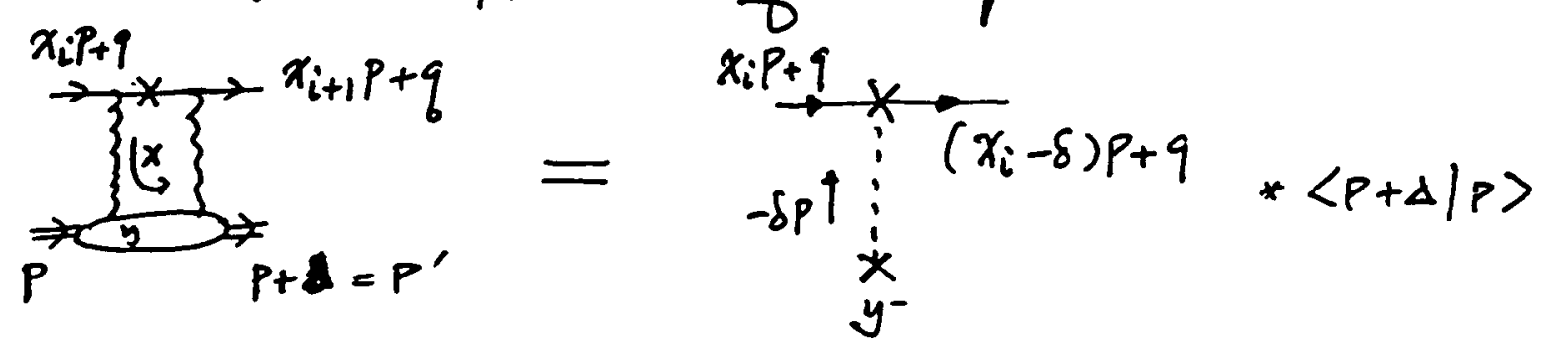

$$
\begin{aligned}
& \overbrace{\left(x_{i}-\delta\right) p+q}^{x_{i} P+q}=(-i)\left(\frac{g^{2}}{2 \pi}\right) r \cdot \bar{n} \operatorname{p} \cdot n\left(\frac{x_{B}}{Q^{2}}\right) \\
& \delta P \downarrow \underset{\substack{\vdots \\
y^{-}}}{\vdots}=(\underbrace{\int p \cdot n d y^{-}}_{\alpha A^{1 / 3}}) K(\delta)\left(\frac{1}{2 N_{c}}\right) \\
& \delta \equiv \frac{\left(P^{\prime}-P\right) \cdot n}{P \cdot n}, K(\delta) \equiv \frac{\left\langle P+\left|A^{\alpha}(0) A^{\beta}(0)\right| P\right\rangle d_{\alpha \beta}}{\langle P+\mid P\rangle}
\end{aligned}
$$

9 
(16)
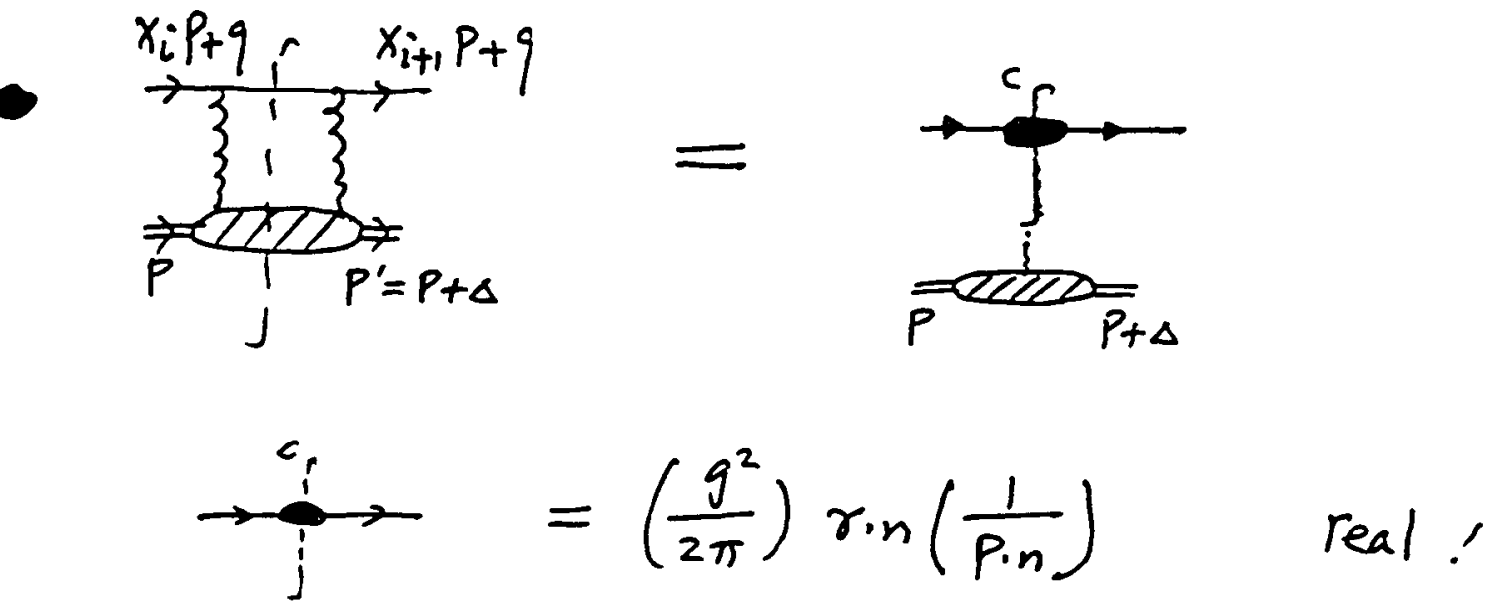

$$
\begin{aligned}
& \frac{: y^{-}}{\sigma_{i}}=(\underbrace{\int p+d y^{-}}_{\alpha A^{1 / 3}})\left[\pi F_{A}\left(x_{B}-\frac{x_{i}+x_{i+1}}{2}, \delta, t\right)\right]\left(\frac{1}{2 N_{c}}\right)
\end{aligned}
$$

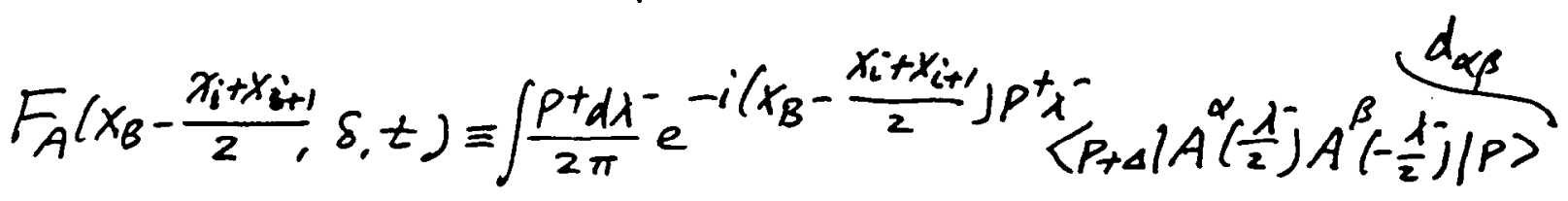

- Skewed parton distribution.

$$
t=P^{\prime}-p=\Delta, \quad \delta \equiv \frac{\left(P^{\prime}-p\right) \cdot n}{p \cdot n}
$$

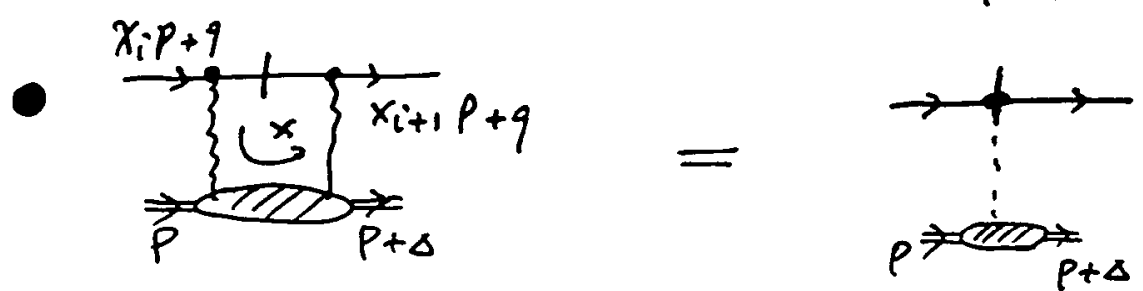

$p+\Delta$

$$
\begin{aligned}
& \rightarrow=\left(\frac{g^{2}}{2 \pi}\right) r \cdot n\left(\frac{1}{p \cdot n}\right)
\end{aligned}
$$

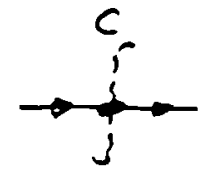

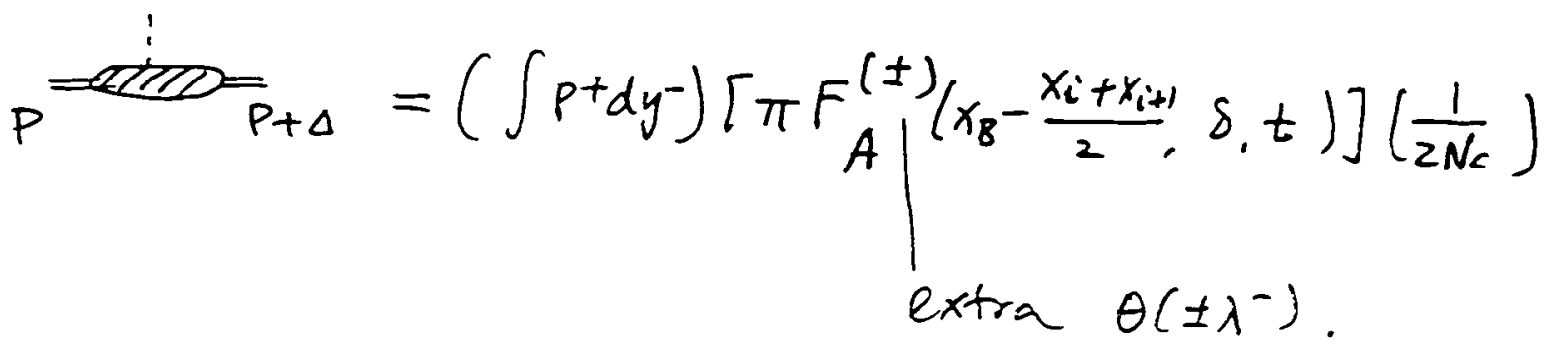

$1 \mathrm{C}$ 
过

* Terms depending on the skewed parton distributions are suppressed.

* Only one-type diagrams contribute to leading $\left(\frac{\alpha_{s} A^{1 / 3}}{Q^{2}}\right)^{m}$ contributions to transverse nuclear structure functions:

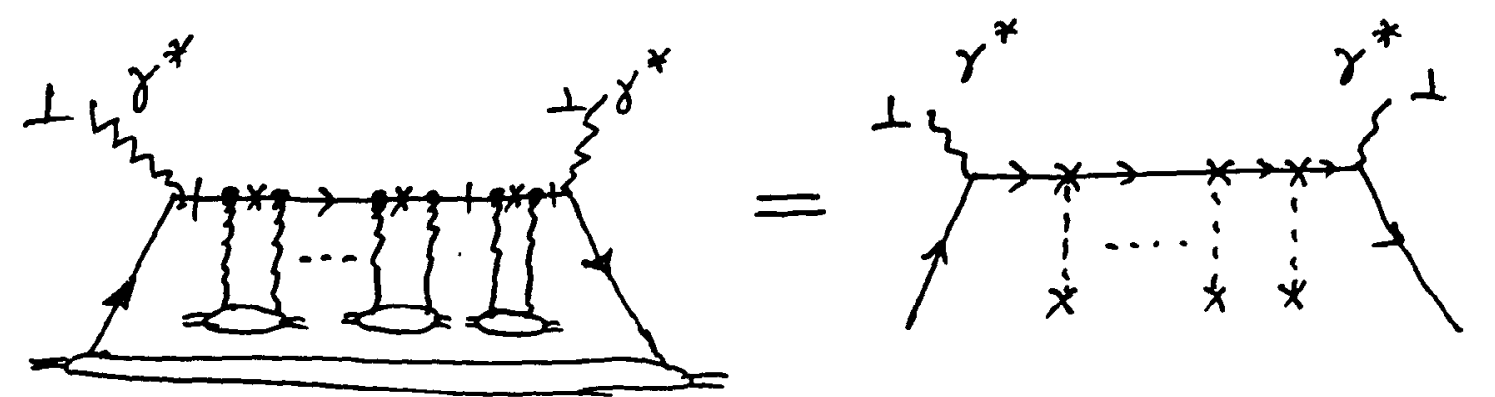

* Leading power and Leading order in $\alpha_{s}:\left(\frac{\alpha_{s} A^{\frac{1}{3}}}{Q^{2}}\right)^{0}$

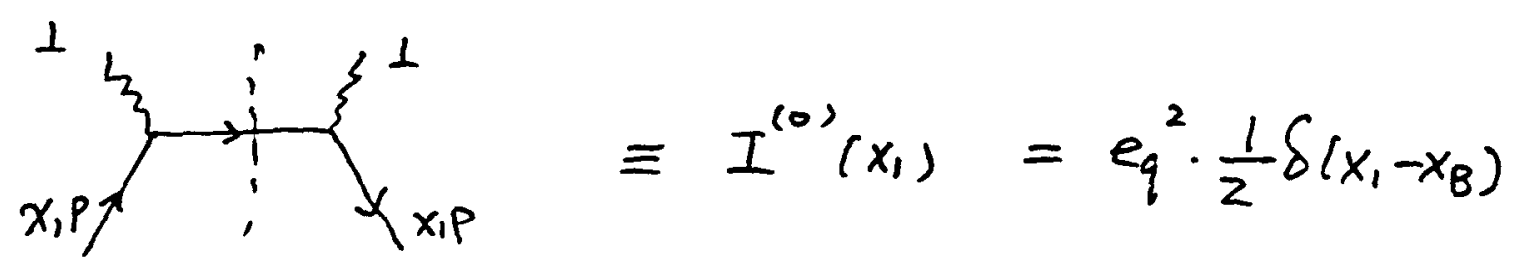

$$
\begin{aligned}
\Rightarrow F_{T}^{A}\left(x_{B}, Q^{2}\right) & =\sum_{q} \frac{1}{2} e_{q}^{2} \int d x_{1} \delta\left(x_{1}-x_{B}\right) q^{A}\left(x_{1}, Q^{2}\right) \\
& =\sum_{q} \frac{1}{2} e_{q}^{2} q^{A}\left(x_{B}, \Phi^{2}\right)
\end{aligned}
$$


(18)

$$
\begin{aligned}
& \text { * NLP: }\left(\frac{\alpha_{s} A^{1 / 3}}{Q^{2}}\right)
\end{aligned}
$$

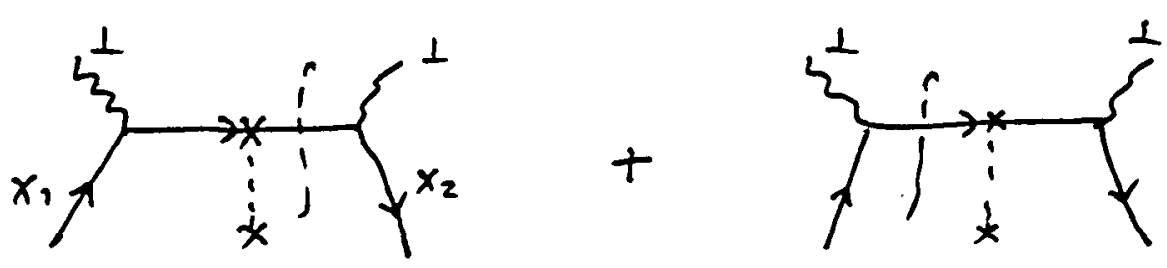

$$
\begin{aligned}
& I^{(\prime)}\left(x_{1}, x_{2}\right)=\left[\frac{I^{(0}\left(x_{1}\right)}{x_{2}-x_{B}}+\frac{I^{(0)}\left(x_{2}\right)}{x_{1}-x_{B}}\right]\left[\frac{g^{2}}{2 \pi} \cdot \frac{x_{B}}{Q^{2}}\right]\left(\int \rho+d y-K(\delta)\right) \\
& +\left(\frac{1}{2 N_{c}}\right) \\
& 1-g^{2} \quad x_{2}=x_{1}-\delta \\
& \Longrightarrow \frac{1}{2} e_{q}^{2}\left[-\delta^{\prime}\left(x_{1}-x_{B}\right)\right]\left[\frac{g^{2}}{2 \pi} \cdot \frac{x_{B}}{Q^{2}}\right]\left(\int p^{+} d y^{-} K\right)\left(\frac{1}{2 N_{C}}\right) \\
& \Rightarrow F_{T}\left(x_{B}, \theta^{2}\right) \mid=\frac{1}{2} \sum_{q} e_{q}^{2} \int d x_{1} q^{A}\left(x_{1}, Q^{2}\right)\left[-\delta^{\prime}\left(x_{1}-x_{B}\right)\right] \\
& \text { * }\left[\frac{g^{2}}{2 \pi} \cdot \frac{X_{B}}{Q^{2}}\right]\left(\int p^{+} d y-K\right)\left(\frac{1}{2 N_{C}}\right) \\
& =\frac{1}{2} \sum_{q} e_{q}^{2} \cdot \Gamma \cdot\left(x_{B} \frac{d}{d x_{B}} q^{A}\left(x_{B}, Q^{2}\right)\right)
\end{aligned}
$$

where:

$$
\begin{aligned}
& \Gamma=2 \frac{\alpha_{s}}{Q^{2}}\left(\frac{1}{2 N_{c}}\right) P\left(\int_{p a t h}^{\left.p p^{+} d y^{-}\right) K} K\right. \\
& \not \equiv \lim _{\delta \rightarrow 0} \frac{\left\langle p+\delta p\left|A^{\alpha}(0) A^{\beta}(0)\right| p\right\rangle}{\langle p+\delta p \mid p\rangle} d_{\alpha \beta}
\end{aligned}
$$

12 
(19)

* NNLP: $\left(\frac{\alpha_{s} A^{1 / 3}}{Q^{2}}\right)^{2}$

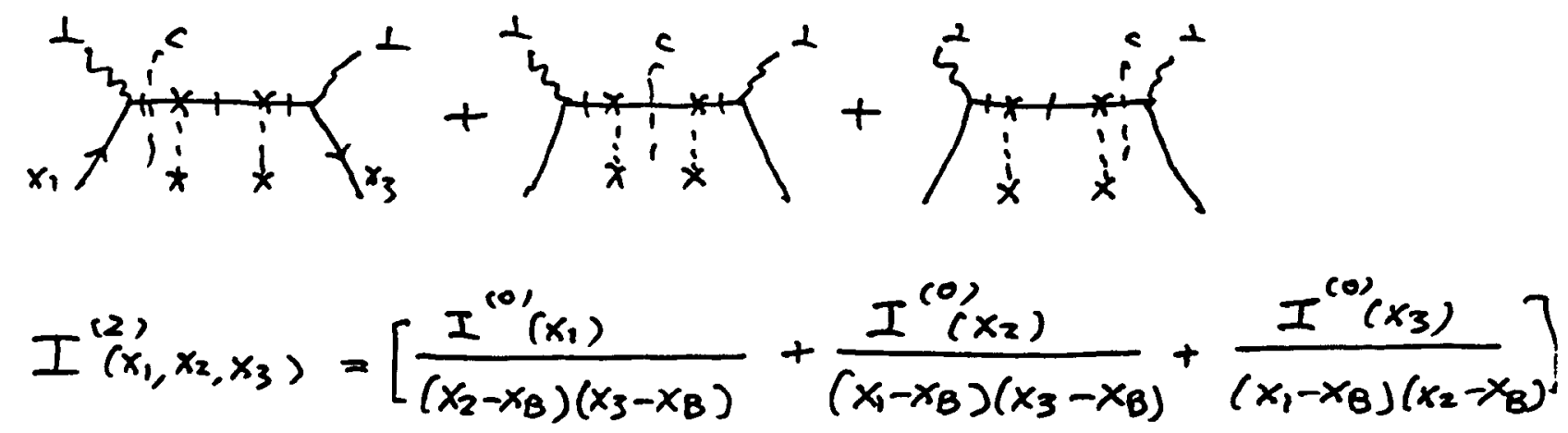

$$
\begin{aligned}
& \text { *P } \left.P \frac{g^{2}}{2 \pi}\left(\frac{x_{B}}{a^{2}}\right) \frac{1}{2 N_{c}}\left(\int p+d y^{-}\right) k(\delta)\right]_{x_{i}=x_{1}-(i-1) \delta}^{2} \\
& \underset{\delta \rightarrow 0}{\Longrightarrow} \frac{1}{2} e_{q}^{2}\left[\frac{(-1)^{2}}{2 !} \delta^{\prime \prime}\left(x_{1}-x_{B}\right)\right] P\left[\frac{g^{2}}{2 \pi}\left(\frac{x_{B}}{Q^{2}}\right)\left(\frac{1}{2 N_{c}}\right) \int P^{+} d j^{-} k\right]^{2} \\
& \Rightarrow F_{T}\left(x_{B}, Q^{2}\right) \mid=\frac{1}{2} \sum_{q} e_{q}^{2} \int d x_{1} q^{A}\left(x_{1}, Q^{2}\right) \frac{\Phi}{2 !}\left[-\left(\frac{g^{2}}{2 \pi}\right)\left(\frac{1}{Q^{2}}\right)\left(\frac{1}{2 N_{C}}\right)\right. \\
& \left.*\left(\int p^{+} d y\right) K\right]^{2} x_{B}^{2} \delta^{\prime \prime}\left(x_{1}-x_{1}\right.
\end{aligned}
$$

* Any given order $\left(\frac{\alpha_{s} A^{1 / 3}}{Q^{2}}\right)^{m}$ :

$$
\begin{aligned}
& I^{(m)}\left(x_{1}, \cdots x_{m+1}\right)=\frac{1}{2} e_{q \rightarrow 0}^{2}\left[\frac{(-1)^{m}}{m !} \delta^{(m)}\left(x_{1}-x_{m}\right)\right] P\left[\frac{g^{2}}{2 \pi}\left(\frac{x_{B}}{Q^{2}}\right)\left(\frac{1}{2 N}\right) \int_{c}^{t} d y K\right]^{m} \\
& \Rightarrow\left[F_{T}^{A}\left(X_{B}, Q^{2}\right)=\frac{1}{2}\right. \\
& \text { *exp }\left\{-2\left(\frac{\alpha_{s}}{Q^{2}}\right)\left(\int P+d y^{-}\right) \frac{k}{2 N_{c}} \cdot x_{B} \frac{d}{d x_{3}}\right\} \delta\left(x_{1}-x_{s} .\right.
\end{aligned}
$$
to $m=\left[A^{1 / 3}\right]$

extra suppression due to the power Corrections. 


\title{
Diffraction and nuclear shadowing
}

\author{
M. Strikman
}

Pennsylvania State University, University Park, PA 16802

\begin{abstract}
A deep connection between diffraction in electron-proton scattering and the nuclear shadowing phenomenon is emphasized. We argue that results of the diffractive studies at HERA allow us to make reliable predictions for shadowing and coherent diffractive cross sections in inclusive $e A$ scattering in a wide range of $x$. Model-independent leading twist expressions for shadowing for the interaction with two nucleons are given. Large shadowing in the gluon channel is predicted based on the current evidence of the dominance of gluons in the "perturbative Pomeron". We demonstrate that so called eikonal models of nuclear shadowing grossly underestimate the amount of shadowing for the interaction of small dipoles, primarily due to the inadequacy of the fixed number of particle approximation. Predictions for the fraction of events in small $x$ electron-nucleus scattering due to coherent and incoherent diffraction are also given.
\end{abstract}

\section{SHADOWING AND DIFFRACTION}

A long time ago V.Gribov [1] investigated the analytic structure of the amplitude for the interaction of highenergy hadrons (photons) with nuclei and established an unambiguous connection between the cross section of small $t$ diffraction of a (virtual) photon or a hadron off the nucleon and the amount of shadowing in the interaction of the projectile with a nucleus in the limit when only two nucleons of the nucleus are involved. For example, for the deuteron of radius $R_{D}$, the effect of nuclear shadowing can be expressed via $\sigma_{\text {shad }}$ as [2] *

$$
\sigma_{\text {shad }}=\frac{\sigma_{t o t}(e D)-2 \sigma_{t o t}(e N)}{\sigma(e N)}=\frac{\left(1-\lambda^{2}\right)}{\left(1+\lambda^{2}\right)} \frac{\frac{d \sigma_{d_{1} / f(e p)}}{d t} \mid t=0}{\sigma_{t o t}(e p)} \frac{1}{8 \pi R_{D}^{2}}
$$

where $\lambda=\operatorname{Re} A / \operatorname{Im} A \approx \frac{\pi}{2} \frac{\partial \ln A}{\partial \ln s}$ for the amplitude of $\gamma^{*} p$ diffractive scattering $A$. For simplicity, we have neglected here the longitudinal form factor which leads to a cutoff of the integral over the diffractive masses, cf. eq. 3 . For small

- In Ref. [1] contribution of the real part was neglected since it was assumed that the total cross section is energy independent. 
$x, \lambda$ may be as large as $0.2-0.3$, leading to $\left(1-\lambda^{2}\right) /\left(1+\lambda^{2}\right) \sim 0.8-0.9$.

It is worth emphasizing that eq. 1 does not require the dominance of the leading twist in diffraction. The only assumption, which is due to a small binding energy of the nucleons, and which is known to work very well in the calculations of hadron-nucleus total and elastic cross sections, is that the nucleus can be described as a multinucleon system ${ }^{\dagger}$. Under these natural assumptions one is essentially not sensitive to any details of the nuclear structure, such as short-range correlations, etc.

\section{INCLUSIVE DIFFRACTION AT HERA AND PREDICTIONS FOR $F_{2 A}$.}

The Gribov approximation has been applied to the description of shadowing in deep inelastic $\gamma^{*} A$ scattering for a long time. First calculations were performed in $[5,6]$ before the advent of HERA and, hence, required modelling of diffraction in DIS. It was performed based on the QCD extension of the Bjorken aligned jet model [5]. These calculations produced a reasonable description of the NMC data. More recently an explicit use of the HERA diffractive data allowed to provide essentially parameter free description of these data [7].

Two important features of the HERA inclusive diffractive data are the approximate Bjorken scaling for the diffractive parton densities and a weak dependence of the total probability of of diffraction $Q^{2}, P_{d i f} \sim 10 \%$ at $Q^{2}>4 \mathrm{GeV}^{2}$.

The first observation is in line with the Collins factorization theorem [8] which states that in the Bjorken limit, the diffractive structure functions $f_{j}^{D}\left(\beta, Q^{2}, x_{\mathbb{P}}, t\right)$ satisfy the DGLAP evolution equations. Here $\beta=x / x_{\mathbb{P}}$.

A relatively small value of the probability of diffraction (as compared to the case of $\pi N$ scattering) indicates that the average strength of the interaction leading to diffraction is relatively small. To characterize this strength it is instructive to treat diffraction in the $S$-channel picture using the eigenstates of the scattering matrix [11], which is complementary to the picture of the factorization theorem. The use of the optical theorem leads to

$$
\sigma_{e f f}^{3}\left(x, Q^{2}\right) \equiv \frac{16 \pi d \sigma^{d i f} / d t}{\sigma_{t o t}\left(x, Q^{2}\right)}
$$

Here the superscript $j$ indicates which hard parton was involved in the elementary hard process of the cross section $\sigma_{\text {tot }}\left(x, Q^{2}\right)$ (i.e. quarks for $F_{2}$ or gluons for $F_{L}$ ). Eq. 2 allows us to extract the average cross section for the

\footnotetext{
†The condition that the matrix element $\left\langle A\left|T\left[J_{\mu}(y), J_{\nu}(0)\right]\right| A\right\rangle$ involves only nucleonic initial and final states 15 not so obvious in the fast frame picture. However, it is implemented in most of the light-cone models [3.4].
} 
configurations which contribute to quark and gluon induced diffraction. It is presented in Fig.1 for $Q^{2}=4 \mathrm{GeV}^{2}$. For larger $Q^{2}, \sigma_{\text {eff }}^{q}$ remains nearly constant, while $\sigma_{\text {eff }}^{g}$ rather rapidly decreases with $Q^{2}$ due to a strong scaling violation for $x g_{N}\left(x, Q^{2}\right)$.

Combining the Gribov theory and the Collins factorization theorem and comparing the QCD diagrams for hard diffraction and for nuclear shadowing due to scattering off two nucleons, one can prove [10] that in the low thickness limit the leading twist nuclear shadowing is unambiguously expressed through the diffractive parton densities $f_{j}^{D}\left(x / x_{\mathbb{P}}, Q^{2}, x_{\mathbb{P}}, t\right)$ of $e p$ scattering:

$$
\begin{array}{r}
f_{j / A}\left(x, Q^{2}\right) / A=f_{j / N}\left(x, Q^{2}\right)-\frac{1}{2}\left(1-\lambda^{2}\right) /\left(1+\lambda^{2}\right) \int d^{2} b \int_{-\infty}^{\infty} d z_{1} \int_{z_{1}}^{\infty} d z_{2} \int_{x}^{x_{0}} d x_{\mathbb{P}} \\
\cdot f_{j / N}^{D}\left(\beta, Q^{2}, x_{\mathbb{P}}, t\right)_{\mid k_{t}^{2}=0} \rho_{A}\left(b, z_{1}\right) \rho_{A}\left(b, z_{2}\right) \cos \left(x_{\mathbb{P}} m_{N}\left(z_{1}-z_{2}\right)\right),
\end{array}
$$

where $f_{j / A}\left(x, Q^{2}\right) a n d f_{j / N}\left(x, Q^{2}\right)$ are the inclusive parton densities; $\rho_{A}(r)$ is the nucleon density in the nucleus. At not too small $x$ one should add a term related to the longitudinal distances comparable to the internucleon distances in nuclei. This additional term can be evaluated using the information on the enhancement of the gluon and valence quark parton densities at $x \sim 0.1$ at the initial $\mathrm{QCD}$ evolution scale $Q_{0}^{2}$. It would slightly diminish nuclear shadowing at higher $Q^{2}$ via the $Q^{2}$ evolution. Thus, for light nuclei for $x \leq 0.01$, the shadowing effect for $1-f_{j / A}\left(x, Q^{2}\right) / A f_{j / N}\left(x, Q^{2}\right) \propto \sigma_{e f f}^{j}\left(x, Q^{2}\right)$.

To evaluate the terms related to the the interactions with $N \geq 3$ nucleons we need to find out what configurations in the photon wave function dominate in diffraction. Two extreme alternatives are the aligned jet model - the dominance of hadron size configurations, and the dominance of configurations of a small size $\sim 1 / Q$. In order to determine which of these extremes is closer to reality we compare the effective cross section characterizing diffraction (eq. 2) with the pQCD result $[12,13]$ for the cross section of the interaction of a small $q \bar{q}$ color singlet dipole of the transverse size $r_{t}{ }^{\ddagger}$ :

$$
\sigma_{q \bar{q}, N}^{i n e l}\left(E_{i n c}\right)=\frac{\pi^{2}}{3} b^{2} \alpha_{s}\left(Q^{2}\right) x G_{N}\left(x, Q^{2} \simeq \frac{\Lambda}{r_{t}^{2}}\right)
$$

where $\Lambda \approx 9$.

A comparison of eqs. 4 and 2 indicates that for the eRHIC range of $Q^{2}, Q^{2} \geq 4 \mathrm{GeV}^{2}$, the large size configurations dominate, while the small dipoles contribute little to the inclusive diffractive cross section. This is consistent with

\footnotetext{
¥The analysis [14] based on eq. 4 which takes into account the taming of the cross section at small $x$ as well as nonperturbative effects at large $r_{\ell} \geq 0.6 \mathrm{fm}$, leads to a reasonable description of the current small- $x$ HERA datia.
} 
the experimental observation that the $t$-slope of the inclusive diffraction $\left(B \sim 7 \mathrm{GeV}^{-2}\right)$ is significantly larger than for the processes where the small size dipole dominate $\left(B \sim 4.5 \mathrm{GeV}^{-2}\right)$. At lower $x \sim 10^{-4}$, the small dipoles may become much more important due to the increase of $\sigma_{q \bar{q}, N}^{i n e l}$ due to the QCD evolution.

It is well known that total, elastic, and inelastic diffractive cross sections for the interactions of hadrons with nuclei are quantitatively described within the the scattering eigenstate approximation which is a generalization of the eikonal approximation. Thus, for $Q^{2}=4 \mathrm{GeV}^{2}$, we considered interactions with $N \geq 3$ nucleons using a generalized eikonal model with $\left\langle\sigma^{2}\right\rangle /\langle\sigma\rangle /=\sigma_{\text {eff }}^{q}$. We found that due to a relatively small value of $\sigma_{\text {eff }}^{q}$, the amount of nuclear shadowing for $F_{2 A}$ is relatively modest and it practically does not depend on the effects of fluctuations of the cross section as soon as $\sigma_{e f f}$ is kept constant [15]. The $Q^{2}$ dependence of shadowing was taken into account via the DGLAP evolution equations. The results of the calculations [10] are presented in Fig.2. Note that since the model of [9] provides a good fit to the HER $\Lambda$ diffractive data for $F_{2}^{D}\left(x, Q^{2}, x_{\mathbb{P}}\right)$, it effectively takes into account higher twist terms, if they are present in the data.

Hence, we conclude that combining the Gribor theory with the factorization theorem for inelastic diffraction and experimental information from HERA provides a reliable prediction for $F_{2 A} / F_{2 D}$ in the eRHIC experimental range.

\section{GLUON SHADOWING}

In perturbative QCD one expects a factor of $9 / 4$ stronger interaction for a color octet dipole than for color triplet dipole $[16,13]$. This leads to a larger probability of perturbative elastic interactions and, hence, to an earlier onset of the regime where unitarity effects may become important. It is straightforward to see based on the unitarity considerations [17] that a growth of $G_{A}\left(x, Q^{2}\right)$ should be tamed already at rather large $x$. Indeed, for the scattering at central impact parameters we find:

$$
\frac{x G_{A}^{\text {cen. zm. par }}\left(x, Q^{2}\right)}{A_{\text {eff }}^{\text {cen. } 2 m \cdot \text { par. }}} \leq 30 A^{-1 / 3}\left(\frac{Q^{2}}{10 G e V^{r^{2}}}\right)=\left(\frac{4 Q^{2}}{10 \mathrm{GeV}^{V^{2}}}\right)_{\mid A=240}
$$

which should be compared, for example, with $x G_{N}\left(x=10^{-3} \cdot Q^{2}=4 G e V^{2}\right) \geq 5$ in the current parton distribution fits. Hence, strong modifications of the gluon field in heary nuclei at the top of the eRHIC kinematics appear unavoidable At the same time, the mechanism of taming is a subject of debates. In principle, taming can emerge in the leading twist via a strong reduction of the nuclear gluon density, or as a combination of the leading twist and higher twist nonlinear effects. 
The leading twist mechanism of taming is connected to the amount of the leading twist diffraction in the gluoninduced reactions. The above mentioned larger strength of the perturbative interaction in the gluon channel as well as stronger nonperturbative interactions in the gluon channel up to the scale $\sim 2 \mathrm{GeV}$ suggest that the gluon-induced diffraction should occur with a large probability.

This is consistent with another interesting feature of the current HERA data (shared by the diffractive data from the proton colliders) - a very important role of the gluons in hard diffraction. In the parlor of the diffractive community, the "perturbative Pomeron" is predominantly built of gluons. To quantify this statement it is convenient to define the probability of diffraction for the action of the hard probe which couples to a parton $j[10]$ :

$$
P_{j}\left(x, Q^{2}\right)=\frac{\int f_{j}^{D}\left(\frac{x}{x_{\mathbb{P}}}, Q^{2}, x_{\mathbb{P}}, t\right) d t d x_{\mathbb{P}}}{f_{j}\left(x, Q^{2}\right)}
$$

A large probability of diffraction in the gluon channel: $P_{g}\left(x \leq 10^{-3}, Q^{2}=4 \mathrm{GeV}^{2}\right) \sim 0.4, P_{g}\left(x \leq 10^{-3}, Q^{2}=\right.$ $10 \mathrm{GeV}^{2} \sim 0.2$ ), leads via eq. 2 to a large $\sigma_{\text {eff }}^{g}$ (Fig.1) and, hence, to the prediction of a large leading twist shadowing for $G_{A}\left(x \leq 10^{-2}, Q^{2}\right)$, see Fig.2. Interactions of small dipoles are more important in the gluon case than in the quark case. Because of this, the question of higher twist effects becomes more complicated for the gluon densities. Also, the gluon induced interactions of a projectile with several nucleons are much more important and more sensitive to details of the interaction dynamics. However, with $\sigma_{\text {eff }}^{g} \sim 40 \mathrm{mb}$, a large leading twist shadowing in the gluon channel appears unavoidable. It seems to be large enough to reduce strongly the gluon densities. However, even this strong reduction does not allow to avoid the violation of unitarity, as given by eq. 5 , for the interaction of color octet systems with heavy nuclei for $Q \sim 2 \mathrm{GeV}$ at $x \leq 5 \cdot 10^{-3}$ [18]. So, for hcavy nuclei some kind of nonlinear dynamics for $Q<2 \mathrm{GeV}$ appears unavoidable.

\section{SHADOWING IN THE INTERACTION OF SMALL DIPOLES WITH NUCLEI}

In several physical situations at small $x$ we can probe directly interactions of a small color dipole with the nucleus. This includes $\sigma_{L}\left(x, Q^{2}\right), \sigma(\gamma+A \rightarrow J / \psi+A)$ and $\sigma\left(\gamma_{L}^{*}+A \rightarrow \rho+A\right)$. One is tempted to calculate shadowing in this case using the eikonal approximation for the fixed transverse size of the dipole, and eq. 4 for the elementary rescattering cross section. In this case, one finds a relatively small shadowing in the eRHIC range both for $\sigma_{L}$ and the vector meson production [17], see Fig.3. However, this approximation is qualitatively inadequate since it violates the QCD factorization theorem [19] which in the case of production of vector mesons at small $x$ leads to [20] 


$$
\frac{\left.\frac{d \sigma}{d t}\left(\gamma^{*} A \rightarrow M A\right)\right|_{t=0}}{\left.\frac{d \sigma}{d t}\left(\gamma^{*} N \rightarrow M N\right)\right|_{t=0}}=\left[\frac{F_{A}^{L}(x, Q)}{F_{N}^{L}(x, Q)}\right]^{2}=\frac{G_{A}^{2}(x, Q)}{G_{N}^{2}(x, Q)} .
$$

To quantify the difference of the strength of shadowing due to the leading twist and due to the eikonal mechanism, we can consider the coherent interaction with two nucleons. The leading twist shadowing via the attachment of two gluons with their subsequent interactions with two nucleons (Fig. 4a) is determined by the strength of the hard gluon-induced diffraction and is governed by the parameter $\sigma_{\text {eff }}^{g}$, see Eq.2. The eikonal screening via the attachment. of four gluons (Fig. $4 \mathrm{~b}$ ) is governed by $\sigma_{q \bar{q} N}$, see Eq.4. We found that for the eRHIC kinematics $\sigma_{e f f}^{g} \gg \sigma_{q \bar{q} N}$, that is, the leading twist shadowing is larger than the eikonal higher twist term by a factor $3 \div 5$. Qualitatively this is due to the QCD evolution which leads to the emission of gluons with virtualities much smaller than $Q^{2}$.

Hence, we predict much larger shadowing for the interaction of small dipoles than suggested within the eikonal models. The cleanest way to observe this effect would be to study coherent $J / \psi$ production. eRHIC would be able to cover the region of the transition from color transparency - the amplitude proportional to $A$, to the regime of perturbative color opacity, see Fig. 5.

\section{TOTAL CROSS SECTION OF COHERENT INCLUSIVE DIFFRACTION}

There is a deep connection between shadowing and phenomenon of diffractive scattering off nuclei. The simplest way to investigate this connection is to apply the AGK cutting rules. Sevcral processes contributc to diffaction on nuclei: (i) Coherent diffraction in which the nucleus remains intact, (ii) Break-up of the nucleus without production of hadrons in the nucleus fragmentation region, (iii) Rapidity gap events with hadron production in the nucleus fragmentation region. In Ref. [2] we found that for $x \leq 3 \cdot 10^{-3}, Q^{2} \geq 4 \mathrm{GeV}^{2}$, the fraction of the DIS events with rapidity gaps reaches the value of about $30-40 \%$ for heavy nuclei, with a fraction of the events of type (iii) rapidly dropping with $A$. Recently, together with M. Zhalov, we investigated the dependence of the fraction of the events due to coherent diffraction and due to the break-up of the nucleus on the strength of the interaction, $\sigma_{\text {eff }}^{J}$. We found

that this fraction increases with $\sigma_{\text {eff }}^{3}$ rather slowly. Thus, it is not sensitive to the fluctuations of $\sigma_{e f f}$. One can see from Fig. 6 that for the quark-induced processes one expects a significantly smaller fraction of coherent events than for the gluon-induced processes. We also found that the ratio of diffraction with the nucleus break-up and with the nucleus remaining intact is small $(10-20 \%)$ in a wide range of nuclei, and weakly depends on $\sigma_{t f f}$. Hence, it would be pretty straightforward to extract coherent diffraction by simply using anti-coincidence with the forward neutron 
detector, especially in the case of heavy nuclei, see discussion in [21].

Other manifestations of the leading twist shadowing include strong $A$-dependence of the fluctuations of the central multiplicity of the produced hadrons [2], and strong modifications of the leading hadron spectrum [10].

\section{CONCLUSIONS}

The nuclear program at eRHIC has a strong potential for the discovery of a number of small $x$ phenomena, including quark and gluon shadowing in the kinematics where average longitudinal distances by far exceed the size of heavy nuclei, hard coherent diffraction off nuclei, color opacity in the production of small color singlets.

[1] V. N. Gribov, Zh. Exp. Teor.Fiz. 571309 (1969) [Sov. Phys. JETP 30709 (1970)].

[2] L.Frankfurt and M.Strikman, Phys.Lett. B382 (1996) 6.

[3] A.H.Mueller and J-W.Qiu, Nucl. Phys. B268 (1986) 427.

[4] L.McLerran and R.Venugopalan, Phys.Rev. D50 (1994) 225.

[5] L. L. Frankfurt and M. I. Strikman, Phys. Rept. 160, 235 (1988).

[6] L. L. Frankfurt and M. I. Strikman, Nucl. Phys. B316, 340 (1989).

[7] A. Capella, A. Kaidalov, C. Merino, D. Pertermann and J. Tran Thanh Van, Eur. Phys. J. C5, 111 (1998)

[8] J.C. Collins, Phys. Rev. D57 (1998) 3051.

[9] L. Alvero, J.C. Collins, and J.J. Whitmore, Phys. Rev. D59, 074022 (1999), hep-ph/9806340, hep-ph/9805268.

[10] L. Frankfurt and M. Strikman, Eur. Phys. J. A5, 293 (1999) [hep-ph/9812322].

[11] E. Feinberg and Ya. Pomeranchuk, Suppl. Nuovo Cimento 111 (1956) 652; M. Good and W. Walker, Phys. Rev. D120 (1960) 1857.

[12] B. Blättel, G. Baym, L.L. Frankfurt and M. Strikman, Phys. Rev. Lett. 71 (1993) 896.

[13] L. Frankfurt, A. Radyushkin, and M. Strikman, Phys. Rev. D55 (1997) 98.

[14] M. McDermott, L. Frankfurt, V. Guzey and M. Strikman, hep-ph/9912547.

[15] L. Alvero, L. L. Frankfurt and M. I. Strikman, Eur. Phys J. A5, 97 (1999) [hep-ph/9810331].

[16] H Abramowicz, L.L. Frankfurt, and M. Strikman DESY 95-047, March 1995; Proceedings of SLAC Summer Inst., 1994, pp. 539-574.

[17] L. Frankfurt, W. Koepf and M. Strikman, Phys. Rev. D54. 3194 (1996) [hep-ph/9509311]. 
[18] L. Frankfurt, V. Guzey, and M. Strikman, in preparation.

[19] J. C. Collins, L. Frankfurt and M. Strikman, Phys. Rev. D56, 2982 (1997) [hep-ph/9611433].

[20] S. J. Brodsky, L. Frankfurt, J. F. Gunion, A. H. Mueller and M. Strikman, Phys. Rev. D50, 3134 (1994) [hep-ph/9402283].

[21] M. Strikman, M. G. Tverskoii and M. B. Zhalov, Phys. Lett. B459, 37 (1999) [nucl-th/9806099].

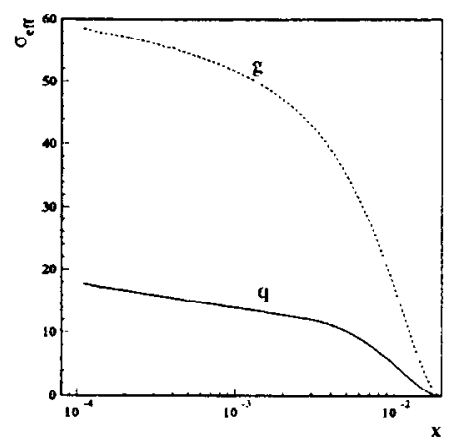

FIG. 1. The $x$-dependence of $\sigma_{e f f}$ for quark- and gluon-induced diffraction for $Q^{2}=4 \mathrm{GeV}^{2}$.
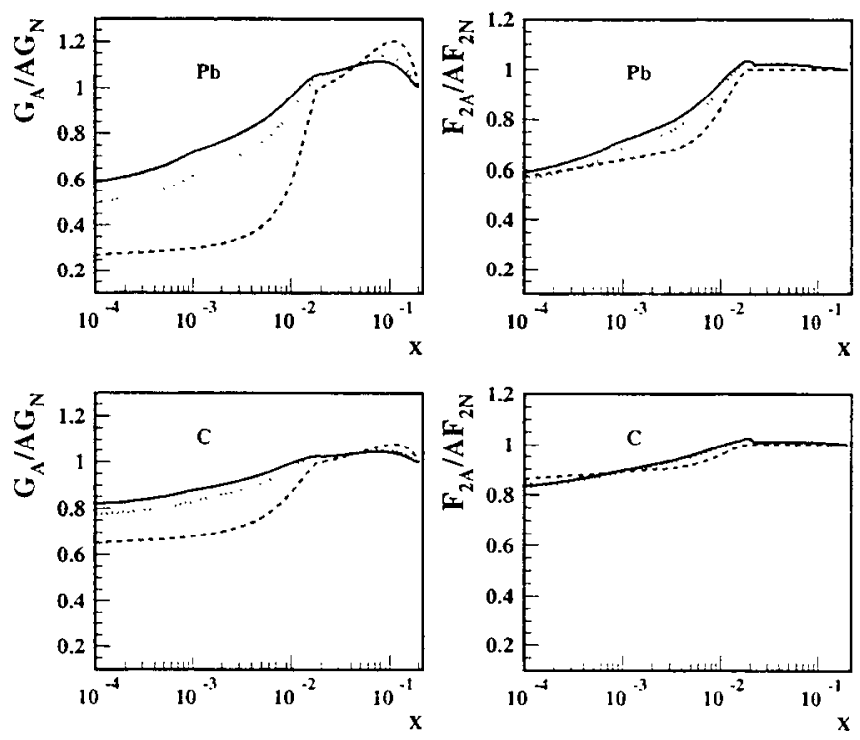

FIG. 2. The dependence of $G_{A} / A G_{N}$ and $F_{2 A} / A F_{2 N}$ on $x$ for $Q=2$ (dashed curve), 5 (dotted curve), and 10 GeV (solid curve) calculated in the quasieikonal model using the diffractive parton densities of [9]. 


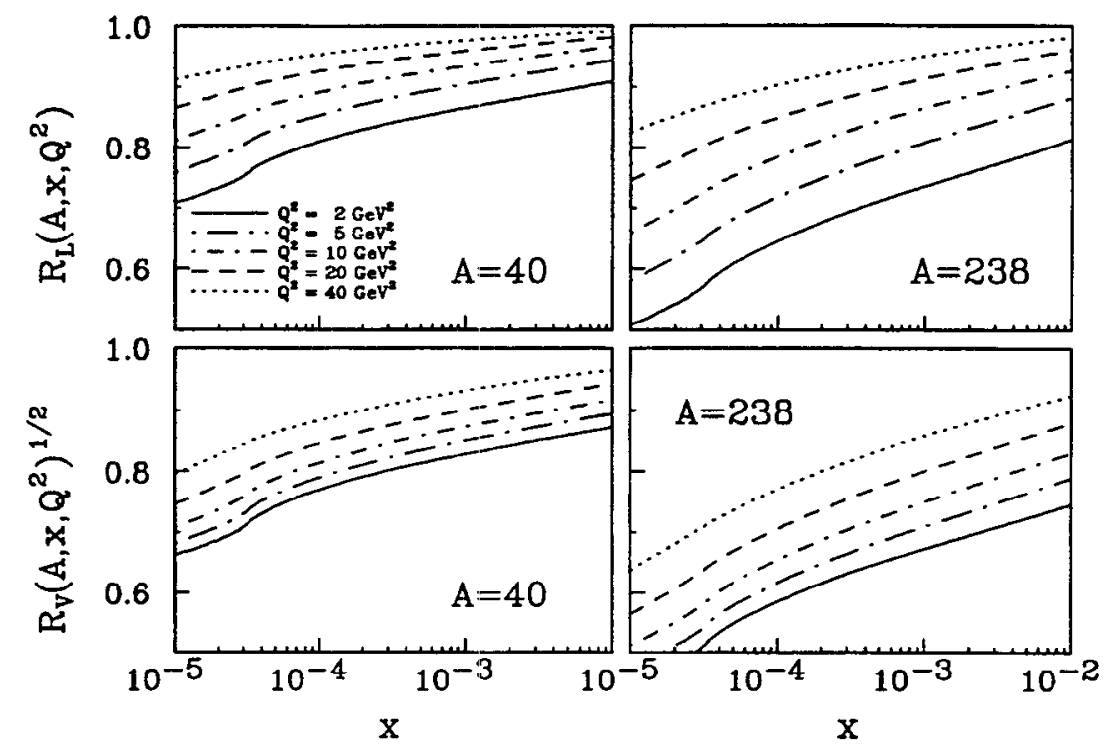

FIG. 3. Nuclear shadowing in DIS in the gluon channel $\left(\sigma_{L}\right)$ and in diffractive electroproduction of $\rho$-mesons is evaluated in $b$-space eikonal approximation. The result for the $J / \psi$ photoproduction corresponds to the $\rho$-meson curve at $Q^{2} \sim 20 \mathrm{GeV}^{2}$.
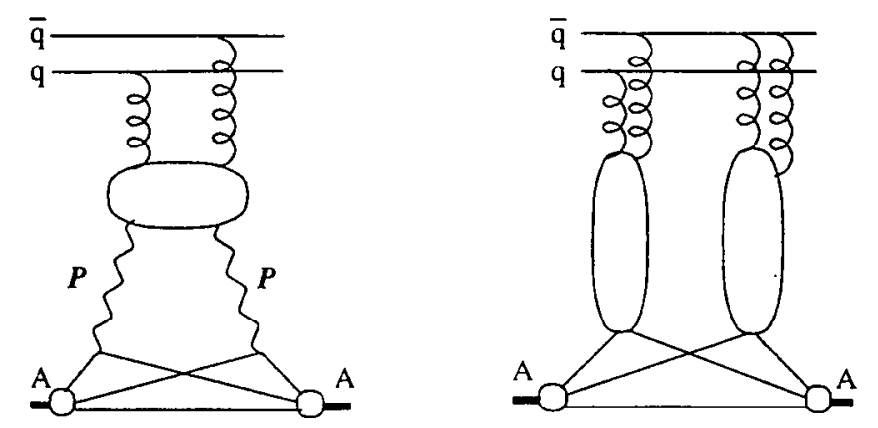

FIG. 4. Typical diagrams for the leading twist (a) and higher twist eikonal interactions (b) of a small dipole with two nucleons. 


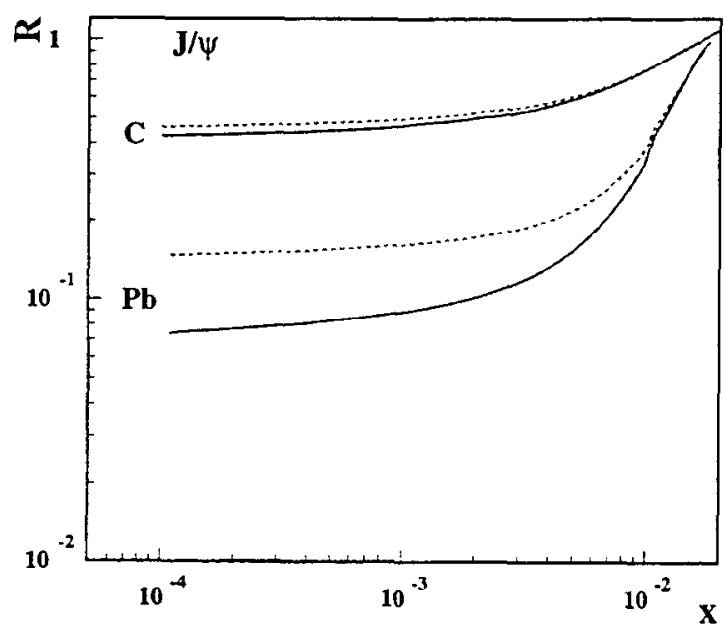

FIG. 5. Color opacity effect for the ratio of the coherent production of $J / \psi$ from carbon (lead) and a nucleon, normalized to the value of this ratio at $x=0.02$ (where color transparency is expected to be valid), calculated in the leading twist approximation with (dashed) and without (solid) account of the fluctuations of the interaction strength.

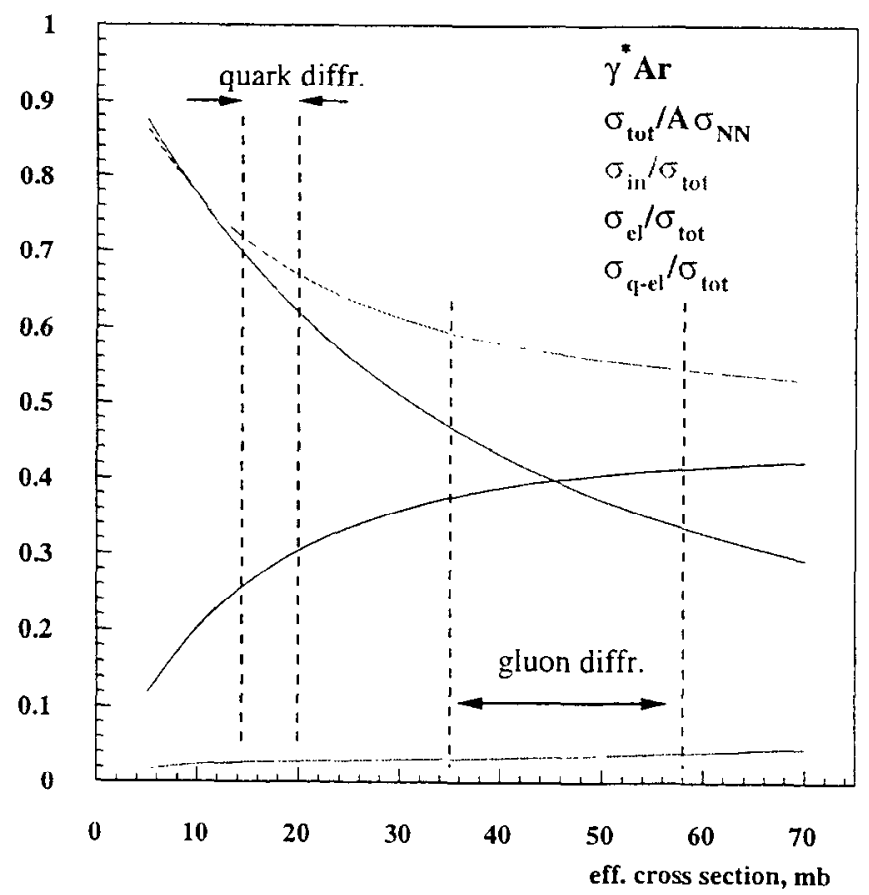

FIG. 6. Dependence of the total cross section, coherent and incoherent diffractive cross sections on the average interaction strength for scattering off lead. 


\title{
Spin Physics Possibilities at eRHIC ${ }^{\dagger}$
}

\author{
M. Stratmann ${ }^{a}$ and W. Vogelsang ${ }^{b}$
}

a Institut für Theoretische Physik, Universität Regensburg, D-93040 Regensburg, Germany

${ }^{b}$ RIKEN-BNL Research Center, Brookhaven National Laboratory, Upton, NY 11973-5000

Polarized $e p$ collisions at eRHIC would provide unique new possibilities for spin physics. First of all, the coverage in Bjorken- $x$ and $Q^{2}$ in polarized DIS would be significantly increased, to $x$ as low as $5 \times 10^{-4}$ and $Q^{2}$ as large as $3 \times 10^{4}$ [1]. This would enormously improve our ability to extract vital information on the nucleon spin structure from polarized DIS. For example, the possibility to go to low $x$ would make determinations of first moments less uncertain. In addition, the lowest- $x\left(x \sim 10^{-3}\right)$ data of present polarized DIS experiments are also at low $Q^{2}\left(Q^{2}<1\right)$; eRHIC would enable measurements at such values of $x$ at much higher $Q^{2}$, where there is less reason to worry about higher-twist contributions. The ability to go to large $Q^{2}$ allows for precise studies of scaling violations in $g_{1}$. Combined analyses of inclusive DIS and two-jet production in DIS would give a clean handle on the polarized gluon density $\Delta g$ [2]. Finally, with sufficient luminosity, it would also become possible to look at electroweak contributions to polarized DIS, which are inaccessbile in fixed-target experiments. Having also positrons and/or neutrons $\left({ }^{3} \mathrm{He}\right)$ available in the beams would be a further important improvement here. For DIS measurements, eR$\mathrm{HIC}$ appears to be generally superior to a possible polarized HERA upgrade (except for the possibility to go to yet lower $x$ and larger $Q^{2}$ at the latter machine [3]), since at given $x$ and $Q^{2}$ the lepton variable $y$ is larger at eRHIC, resulting in a more favorable photon depolarization factor [1].

An even more unique application of eRHIC would be to study the parton content of polarized quasi-real photons. The parton distributions of circularly polarized photons, defined as

$$
\Delta f^{\gamma}\left(x, Q^{2}\right) \equiv f_{+}^{\gamma_{+}}\left(x, Q^{2}\right)-f_{-}^{\gamma_{+}}\left(x, Q^{2}\right)
$$

where $f_{+}^{\gamma_{+}}\left(f_{-}^{\gamma_{+}}\right)$denotes the density of a parton $f$ with helicity ' + ' ('-') in a photon with positive helicity, are completely unmeasured so far. Studies $[5,6]$ have shown that polarized HERA could be in the position to shed some light on

\footnotetext{
'Talk presented by $\mathbb{W}$. Vogelsang
} 
the $\Delta f^{\gamma}$, the preferred tool being the study of 'resolved'-photon contributions to the spin asymmetry for (di-)jet photoproduction. For this workshop, the previous HERA studies have been extended to the case of eRHIC with $\sqrt{s_{\mathrm{ep}}}=100$ $\mathrm{GeV}$. As $[5,6]$, our study employs two very different models for the $\Delta f^{\gamma}[7]$, and examines the sensitivity of di-jet production to these unknown quantities. Unfolding the $\Delta f^{\gamma}$ from a jet-measurement would in general be a very involved task, since many subprocesses and combinations of parton densities contribute to the cross section. To lowest order, the situation can be simplified by employing the 'effective parton density' approximation developed and exploited in $[8,6]$.

The lower energy of eRHIC, as compared to the HERA situation, has both advantages and disadvantages: spin asymmetries are generally expected to be larger at eRHIC since - for a given jet transverse momentum - the parton densities are probed at somewhat higher $x$. On the other hand, event rates will be lower, with ensuing larger statistical uncertainties (under the assumption of similar integrated luminosities for polarized HERA and eRHIC). Also, the 'resolved-photon' component we are interested in here generally becomes the less important, the smaller the energy of the interaction is. Nevertheless, our results show the trend that the advantages of having lower energies outweigh the disadvantages, so that eRHIC appears superior to HERA also concerning the potential for determining the $\Delta f^{\gamma}$.

Among further topics for spin physics at eRHIC would be a measurement of the transversity densities via the Collins effect [4].

\section{References}

[1] see, e.g., A. Deshpande, talk presented at the ' $2^{\text {nd }} e$ RHIC Workshop', Yale, April 2000.

[2] see. e.g., G. Rädel, talk presented at the '2 $2^{\text {nd }}$ eRHIC Workshop', Yale, April 2000.

[3] see, e.g., A. de Roeck, talk presented at the '2 ${ }^{\text {nd }}$ eRHIC Workshop', Yale, April 2000.

[4] see, e.g., M. Grosse-Perdekamp, talk presented at the '2 $2^{\text {nd }}$ eRHIC Workshop', Yale, April 2000 .

[5] M. Stratmann and IV. Vogelsang. Z. Phys. C74 (1997) 641; hep-ph/9608408;

J.M. Butterworth. N. Goodman. M. Stratmann, and W. Vogelsang, hep-ph/9711250.

[6] M. Stratmann and WV. Togelsang. hep-ph/9907470.

[7] M. Glück and II. Vogelsang; Z. Phys. C55 (1992) 353; ibid. C57 (1993) 309:

M. Glük. M. Stratmann. and IT. Togelsang, Phys. Lett. B337 (1994) 373.

[8] B.L. Combridge and C.J. Maxwell. Nucl. Phys. B239 (1984) 429 :

H1 collab.: C. Adluff et al. Eur. Phys. J. C1 (1998) 97. 


\section{eRHIC $g_{1}^{\mathrm{p}}$ data}

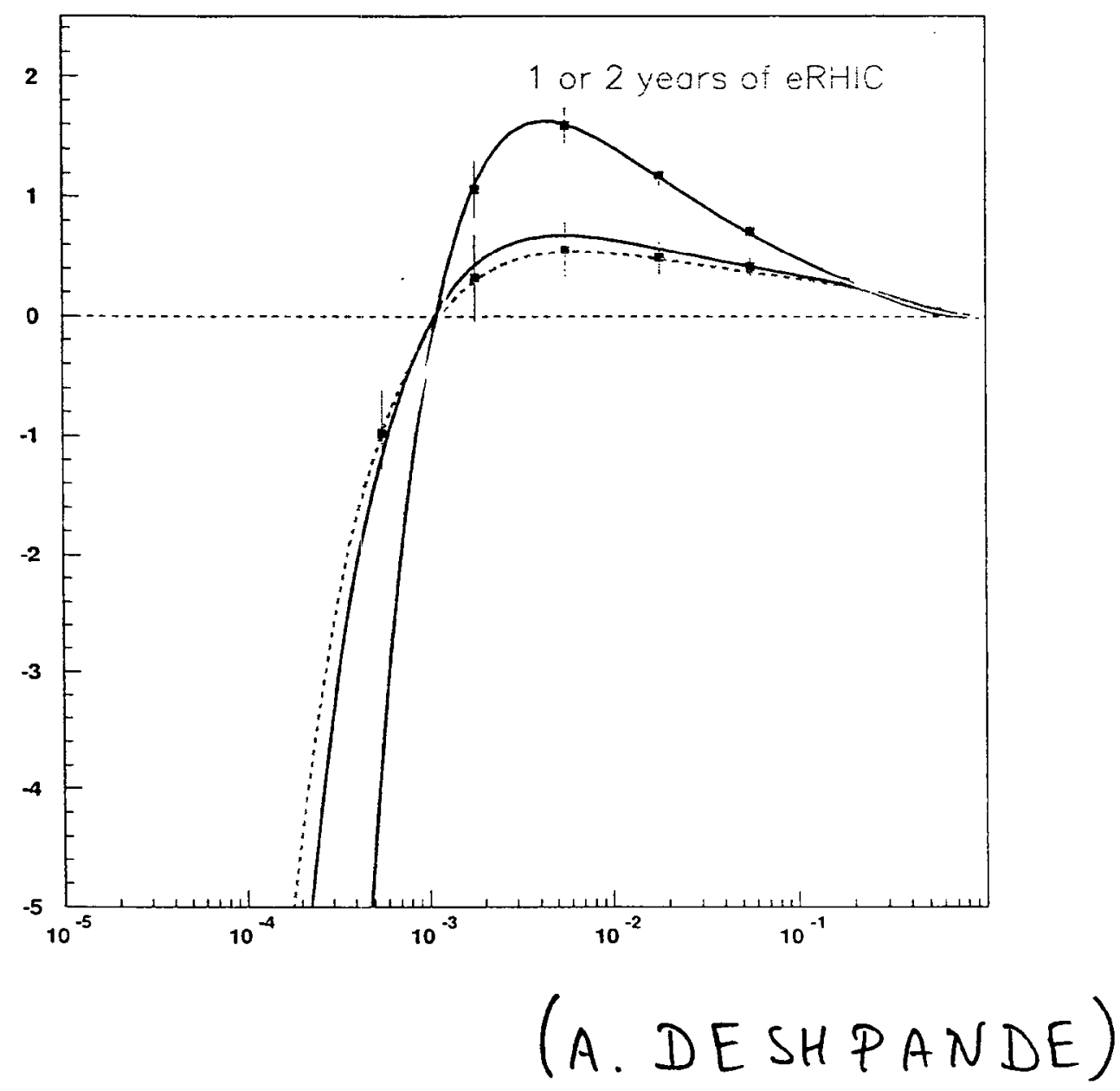




\section{G.RAD}

\section{$\Delta \boldsymbol{G}$ FROM DI-JET EVENTS}

$\star \Delta G$ FROM NLO FITS TO $g_{1}$

$\star \Delta \boldsymbol{G}$ FROM 2-JET EVENTS (LO)
"INDIRECT"

"DIRECT"

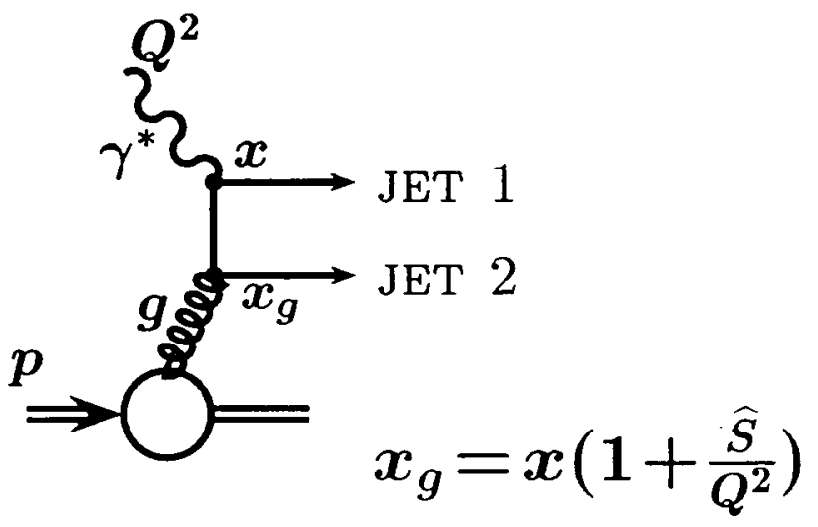

"BOSON GLUON FUSION (BGF)"

$$
\text { IN LO: } \boldsymbol{\sigma} \sim \boldsymbol{G}
$$

SIGNAL

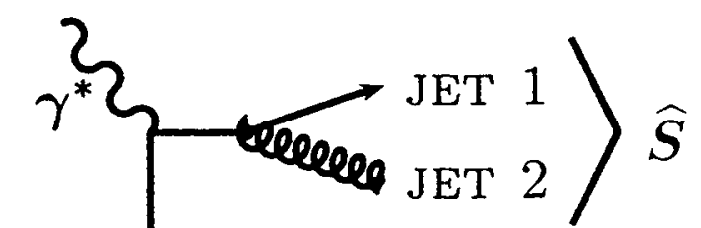

CARGIIZ, MUECLER

ALTARELLI, S MANOHA "QCD-COMPTON"

$$
\boldsymbol{\sigma} \sim \boldsymbol{q}
$$

BACKGROUND

$\star$ AT LOW $\boldsymbol{x}$ BGF DOMINATES

$\star$ PRocess used at hera to eXtract $\boldsymbol{G}(\boldsymbol{x})$ in lo

$\star$ USE THIS METHOD FOR POLARISED CASE:

$$
\begin{gathered}
A_{2 j e t s}=\frac{\sigma_{2 j e t s}^{\uparrow \uparrow}-\sigma_{2 j e t s}^{\uparrow \uparrow}}{2 \sigma_{2 j e t s}}=\mathcal{A} \frac{\Delta G}{G} \frac{\sigma_{2 j e t s}^{B G F}}{\sigma_{2 j e t s}}+\mathcal{B} \frac{\Delta q}{q} \frac{\sigma_{2 j e t s}^{Q C D C}}{\sigma_{2 j e t s}} \\
A_{\text {meas }}=\frac{N_{2 \text { jets }}^{\uparrow \downarrow}-N_{2 j e t s}^{\uparrow \uparrow}}{N_{2 j e t s}^{\uparrow \downarrow}+N_{2 j e t s}^{\uparrow \uparrow}}=P_{e} P_{p} D A_{2 j e t s} \stackrel{\propto}{\sim} \frac{\Delta G}{G}
\end{gathered}
$$




\section{SENSITIVITY TO $\Delta G$}
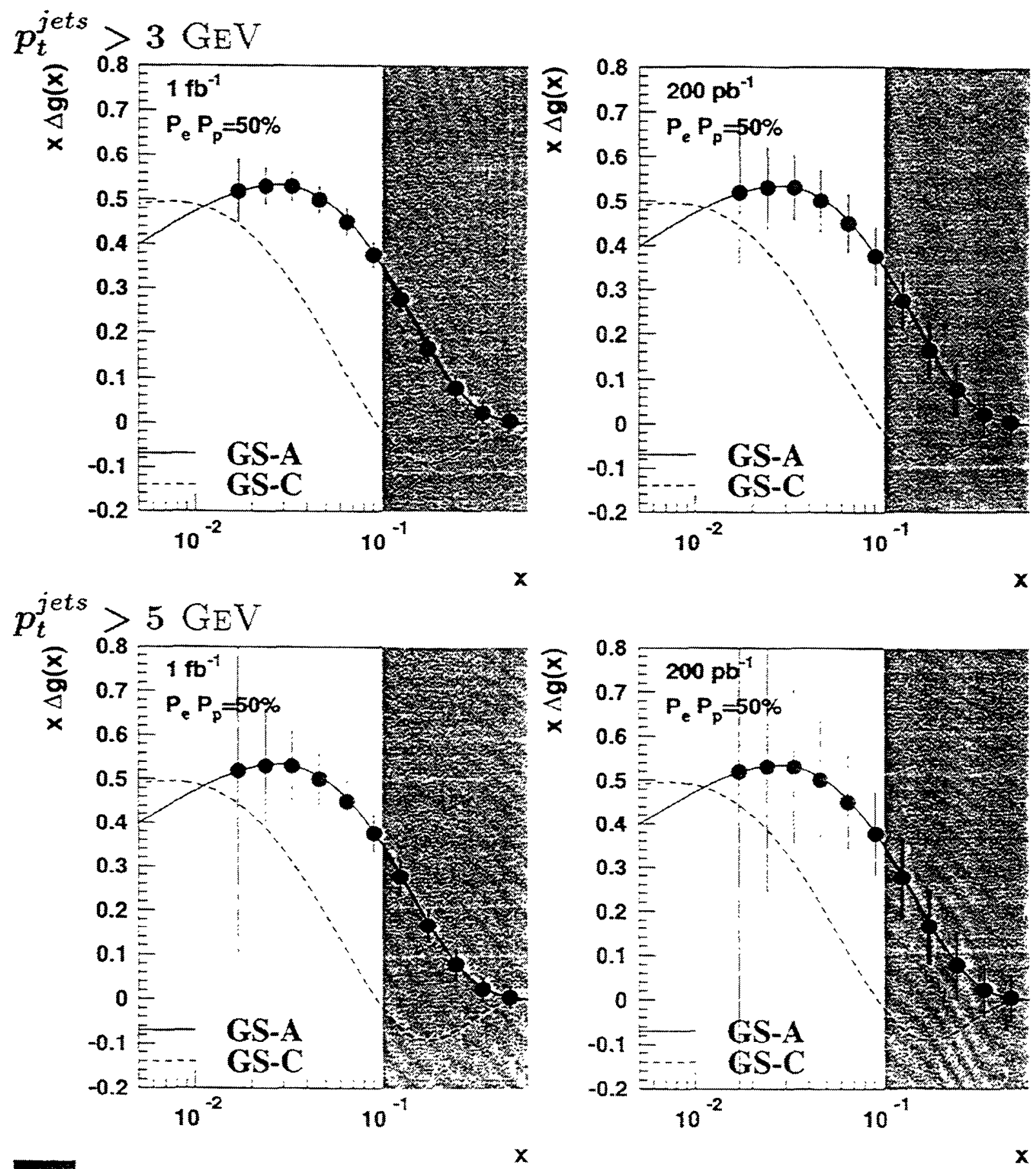

ASYMMETRIES DLE TO QCD-C ARE DOMINATING!

$\star$ GOOD SENSITIVIY TO $\Delta G$ FOR $0.02 \lesssim x_{g} \lesssim 0.1$

$p_{t}^{\text {jets }}>5 \rightarrow 3 \mathrm{CEV}>\mathrm{STATISTICS}$

ESPECIALLY IMPORTAXI FOR $x_{g} \lesssim 0.04$ 
Charged-current DIS ? (no studies yet)

$$
\begin{aligned}
& A_{c c}^{W^{-}}=\frac{2 b g_{1}^{\left(W^{-}\right)}+a g_{5}^{\left(W^{-}\right)}}{a F_{1}^{\left(W^{-}\right)}+b F_{3}^{\left(W^{-}\right)}} \\
& a=2\left(y^{2}-2 y+2\right), b=y(2-y) \\
& g_{1}^{\left(W^{-}\right)}=\Delta u+\Delta \bar{d}+\Delta \bar{s} \pm \ldots \\
& g_{1}^{\left(W^{+}\right)}=\Delta d+\Delta \bar{u}+\Delta s \pm \ldots \\
& g_{5}^{\left(W^{-}\right)}=\Delta u-\Delta \bar{d}-\Delta \bar{s} \pm \ldots \\
& g_{5}^{\left(W^{+}\right)}=\Delta d-\Delta \bar{u}+\Delta s \pm \ldots \\
& \text { with }{ }^{3} \text { He, would get neutron sfs. ! }
\end{aligned}
$$

Examples (non-singlet):

$$
\begin{aligned}
& g_{1}^{\left(W^{-}\right) p}-g_{1}^{\left(W^{+}\right) p}=\Delta u_{v}-\Delta d_{v} \\
& g_{5}^{\left(W^{+}\right) p}+g_{5}^{\left(W^{-}\right) p}=\Delta u_{v}+\Delta d_{v} \\
& g_{5}^{\left(W^{+}\right) p}-g_{5}^{\left(W^{-}\right) n}=-\mathcal{A}_{3}
\end{aligned}
$$

rich information! Also integrals:

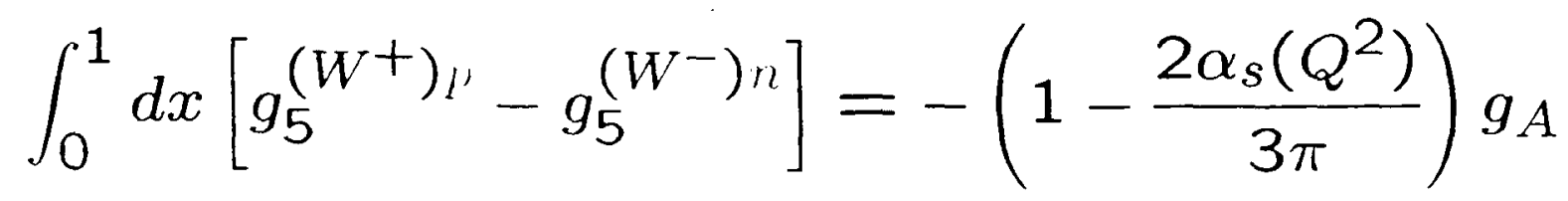
as fundamental as Bj sum rule! (Stratmann,WV) 


\section{Polarized-Photon Pdfs: Models}

Hadronic component ('homogeneous problem')

$\Rightarrow$ non-pert. boundary condition ('input')

- usually VMD ideas

- GRV : at low input scale ( $\mu \sim 0.5 \mathrm{GeV})$ assume photon behaves like VM

$q^{\gamma}\left(x, \mu^{2}\right)=\frac{4 \pi \alpha_{e m}}{\partial \ell f_{\rho}^{2}} q^{\rho}\left(x, \mu^{2}\right) \equiv \frac{4 \pi \alpha_{e m}}{\not \ell f_{\rho}^{2}} q^{\pi}\left(x, \mu^{2}\right)$

(same for $g^{\gamma}\left(x, \mu^{2}\right)$ )

everything else from evolution!

- polarized case : input not so obvious 'benchmark scenarios' (cf. $\left|\Delta q^{\gamma}\right| \leq q^{\gamma}$ ):

(Glück,Stratmann,WV)

'maximal' $\quad \Delta q^{\gamma}\left(x, \mu^{2}\right)=q^{\gamma}\left(x, \mu^{2}\right)$

'minimal' $\Delta q^{\gamma}\left(x, \mu^{2}\right)=0$

(same for $\Delta g^{\gamma}\left(x, \mu^{2}\right)$ ) 


\section{Processes of interest:}

- single-inclusive jet production, $e p \rightarrow$ jet $X$
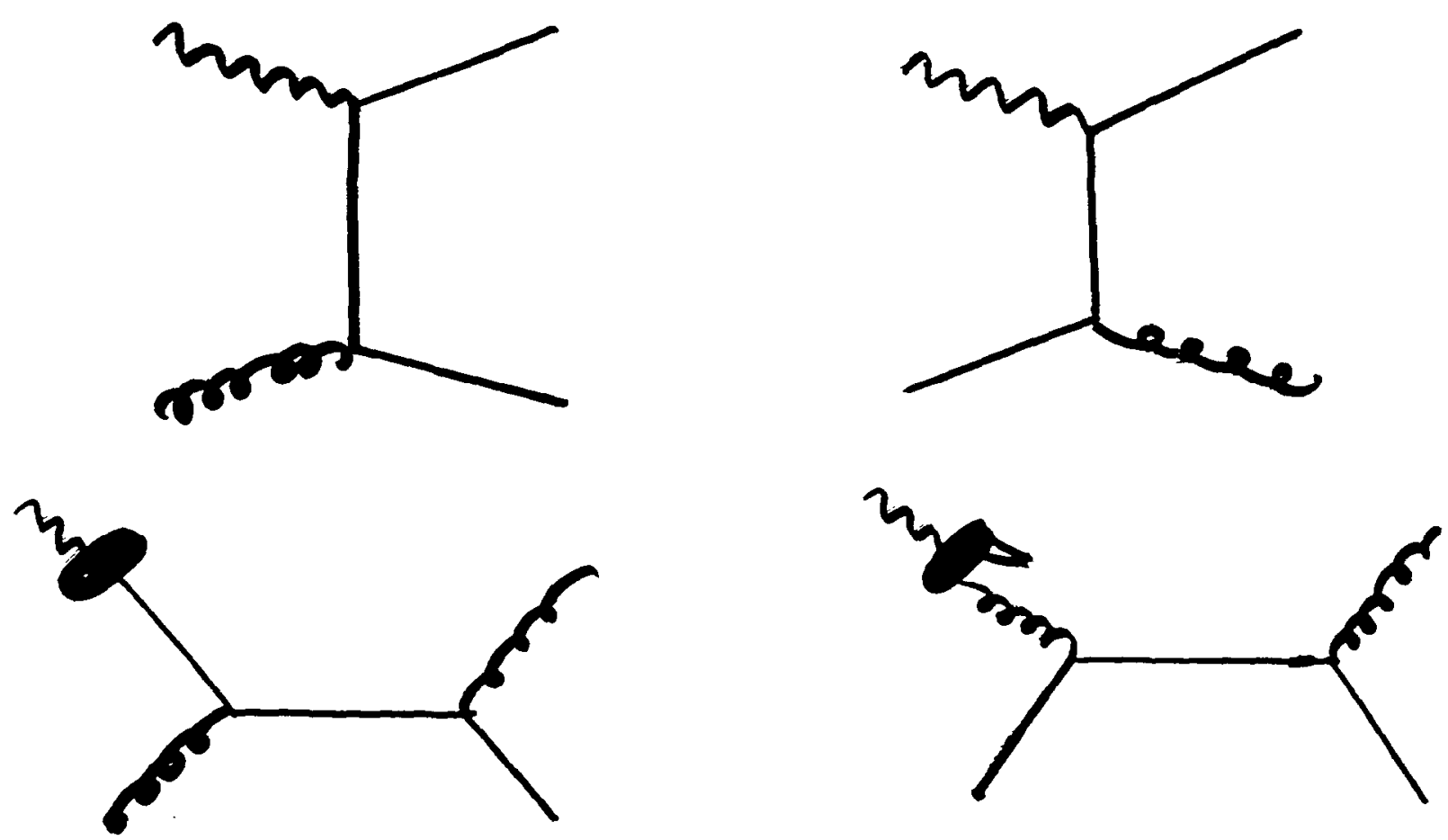

$d \sigma_{\mathrm{dir}}^{\mathrm{jet}} \approx \sum_{f, c, d} \int_{\otimes} f^{p}\left(x_{p}\right) d \widehat{\sigma}_{\gamma f \rightarrow c d}$

$d \sigma_{\text {res }}^{\text {jet }} \approx \sum_{f_{1}, f_{2}, c, d} \int_{\otimes} f_{1}^{\gamma}\left(x_{\gamma}\right) f_{2}^{p}\left(x_{p}\right) d \widehat{\sigma}_{f_{1} f_{2} \rightarrow c d}$

"direct" dominates at $\eta^{\text {jet }}<0: x_{\gamma} \approx 1$

$\rightarrow \Delta g$ from photon-gluon fusion

"resolved" dominates at $\eta^{\text {jet }}>0: \rightarrow \Delta f^{\gamma}$

- similar mechanism for single-inclusive hadrons, $e p \rightarrow h X \quad(h=\pi, K, \ldots)$ 
- dijet production : $p_{T}, \eta_{1}, \eta_{2} \stackrel{\llcorner O}{\longleftrightarrow} x_{p}, x_{\gamma}$ Define

$$
x_{\gamma}^{\mathrm{obs}} \equiv \frac{1}{2 y E_{e}} \sum_{\mathrm{jets}} E_{T}^{\mathrm{jet}} e^{-\eta^{\mathrm{jet}}}
$$

and select, e.g., $x_{\gamma}^{\text {obs }}<0.75$ :

$\longleftrightarrow$ resolved component

For first look : "effective parton densities"

(unpol. case : Forshaw, Roberts;

Combridge, Maxwell; H1)

$$
d \sigma_{\text {res }}^{2-\mathrm{jet}} \approx \int_{\otimes} f_{\mathrm{eff}}^{\gamma} f_{\mathrm{eff}}^{p} d \widehat{\sigma}_{q q^{\prime} \rightarrow q q^{\prime}}
$$

where

$$
f_{\mathrm{eff}}^{(p, \gamma)} \equiv \sum_{q}\left[q^{(p, \gamma)}+\bar{q}^{(p, \gamma)}\right]+\frac{9}{4} g^{(p, \gamma)}
$$

similar approach possible in polarized case:

$$
\Delta f_{\mathrm{eff}}^{(p, \gamma)} \equiv \sum_{q}\left[\Delta q^{(p, \gamma)}+\Delta \bar{q}^{(p, \gamma)}\right]+\frac{11}{4} \Delta g^{(p, \gamma)}
$$

(Indumathi, Murthy, Ravindran; Stratmann,WV; 
- Di-jets $\bullet \vec{e} \vec{p}$ machines: overview \& comparison
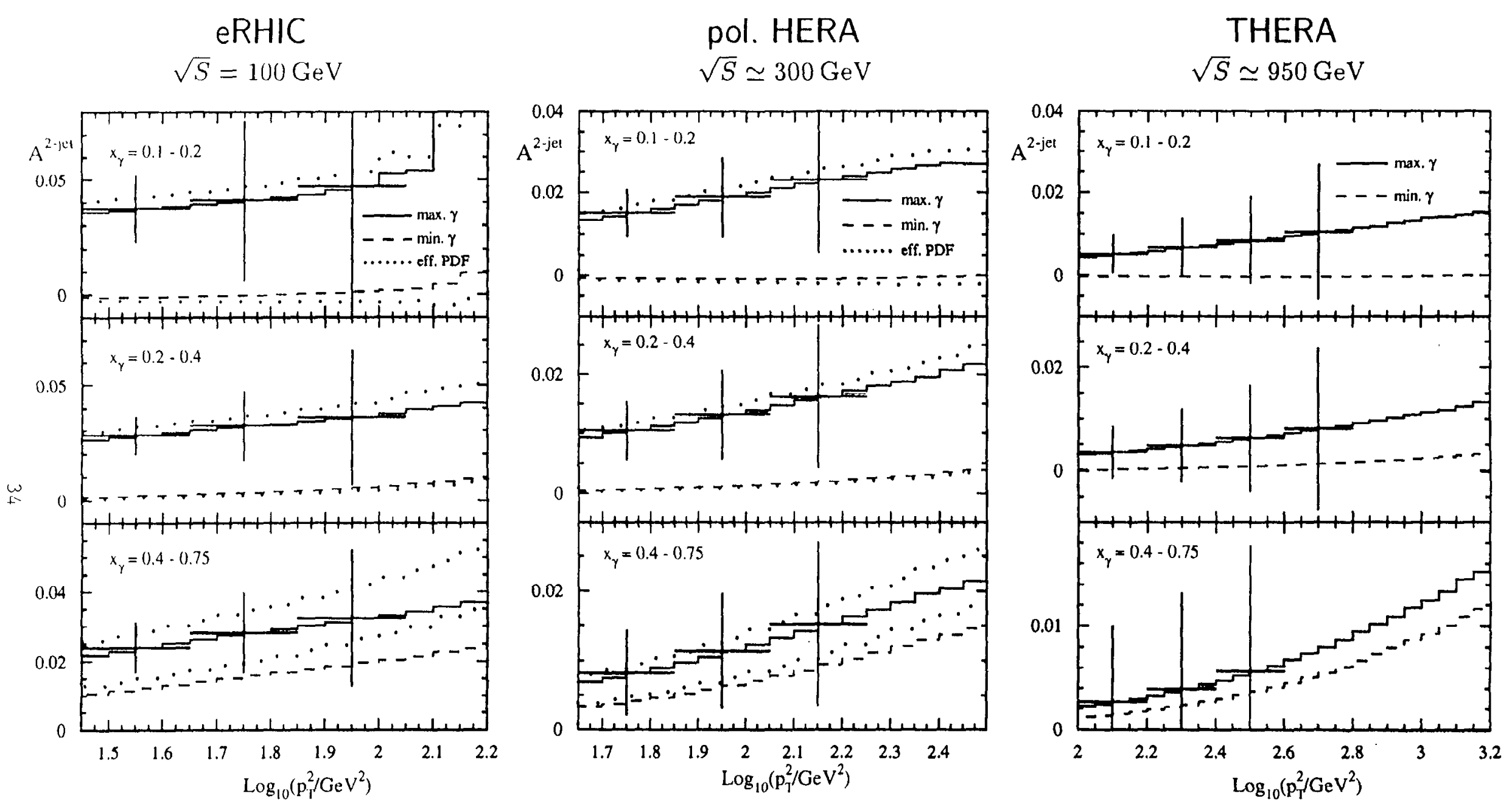

param. used: LO; $\Delta f^{p}:$ GRSV; $\mu=p_{T}$; 'errors': $\mathcal{L}=200 \mathrm{pb}^{-1}, P_{e}=P_{p}=0.7 ; W W: 0.2 \leq y \leq 0.85, Q_{\max }^{2}=4(1) \mathrm{GeV}^{2}$ (eRHIC) $|\Delta \eta| \equiv\left|\eta_{1}-\eta_{2}\right| \leq 1,0<\frac{\eta_{1}+\eta_{2}}{2}<2$ (eRHIC, pol. HERA) and $-1.5<\frac{\eta_{1}+\eta_{2}}{2}<1.5$ (THERA) 
- 1-jet inclusive @ $\vec{e} \vec{p}$ machines: overview \& comparison

\section{eRHIC \\ $\sqrt{S}=100 \mathrm{GeV}$}

$\stackrel{\omega}{\omega}$

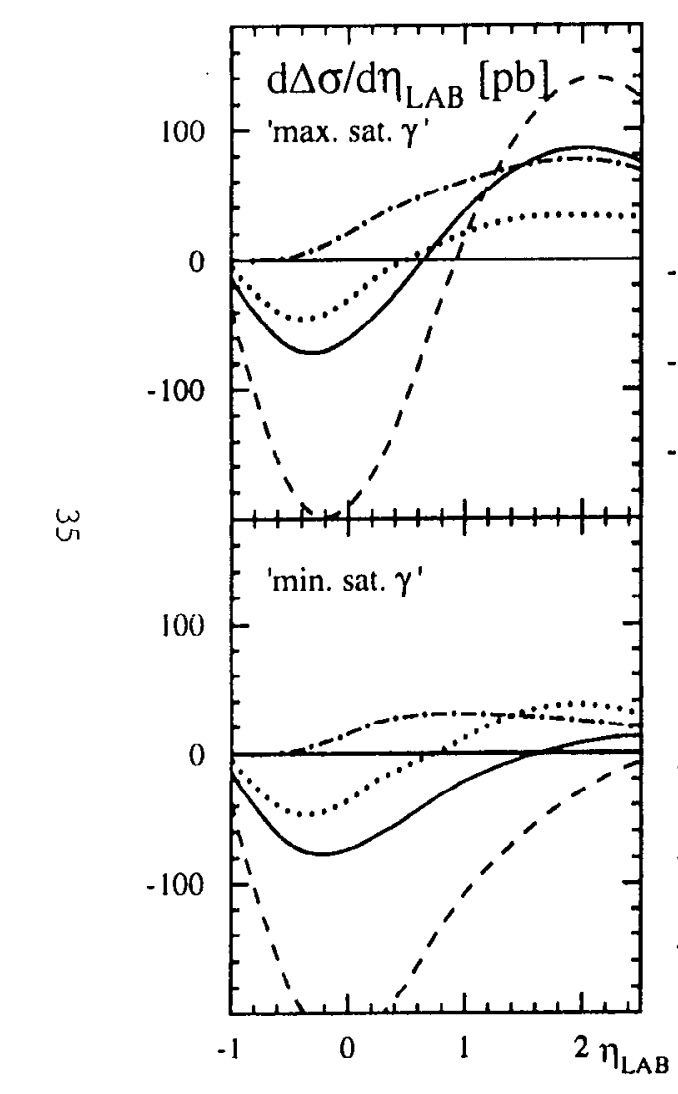

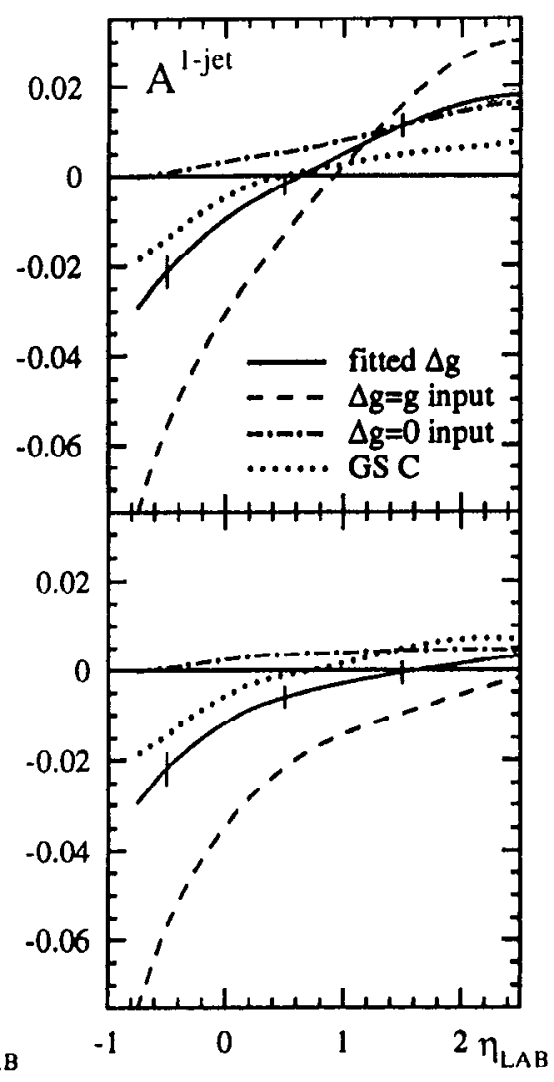

pol. HERA

$\sqrt{S} \simeq 300 \mathrm{GeV}$
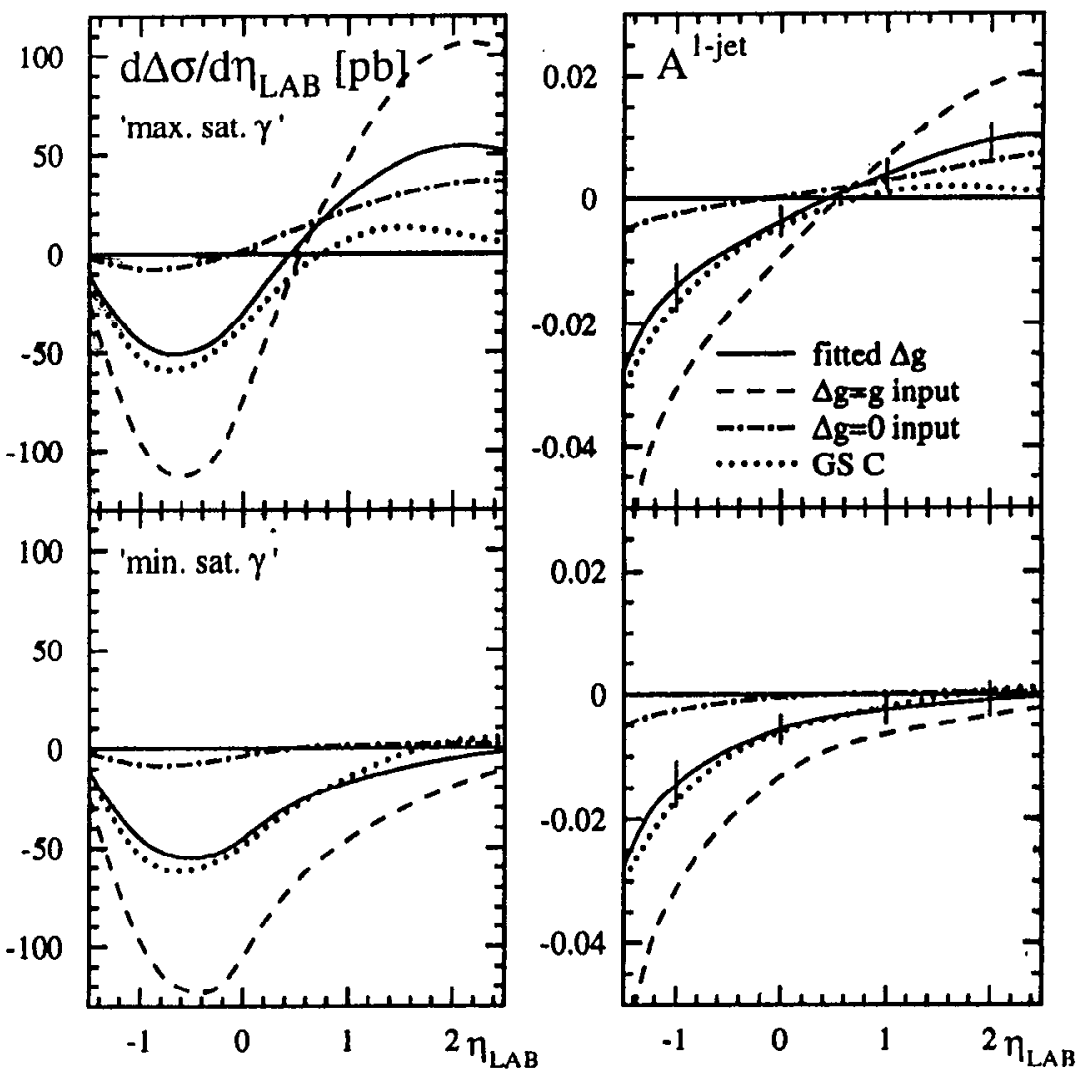

param. used: LO; $\Delta f^{p}$ : GRSV; $\mu=p_{T}$; 'errors': $\mathcal{L}=100 \mathrm{pb}^{-1}, P_{e}=P_{p}=0.7$

$W W: 0.2 \leq y \leq 0.85, Q_{\max }^{2}=4(1) \mathrm{GeV}^{2}(\mathrm{eRHIC}) ; p_{T}^{\min }=8(5) \mathrm{GeV}(\mathrm{eRHIC})$ 


\section{GROSSE - PERDE KAMP}

\section{Case II+}

$\begin{gathered}\text { Detector Acceptance } \\ -2.0<\eta<1.0 \\ \text { Integrated Luminosity } \\ 200 p b^{-1}\end{gathered}$
Cuts: $Q^{2}>1 \mathrm{GeV}^{2}$
$x_{\mathrm{F}}>0$
$z>0.1$
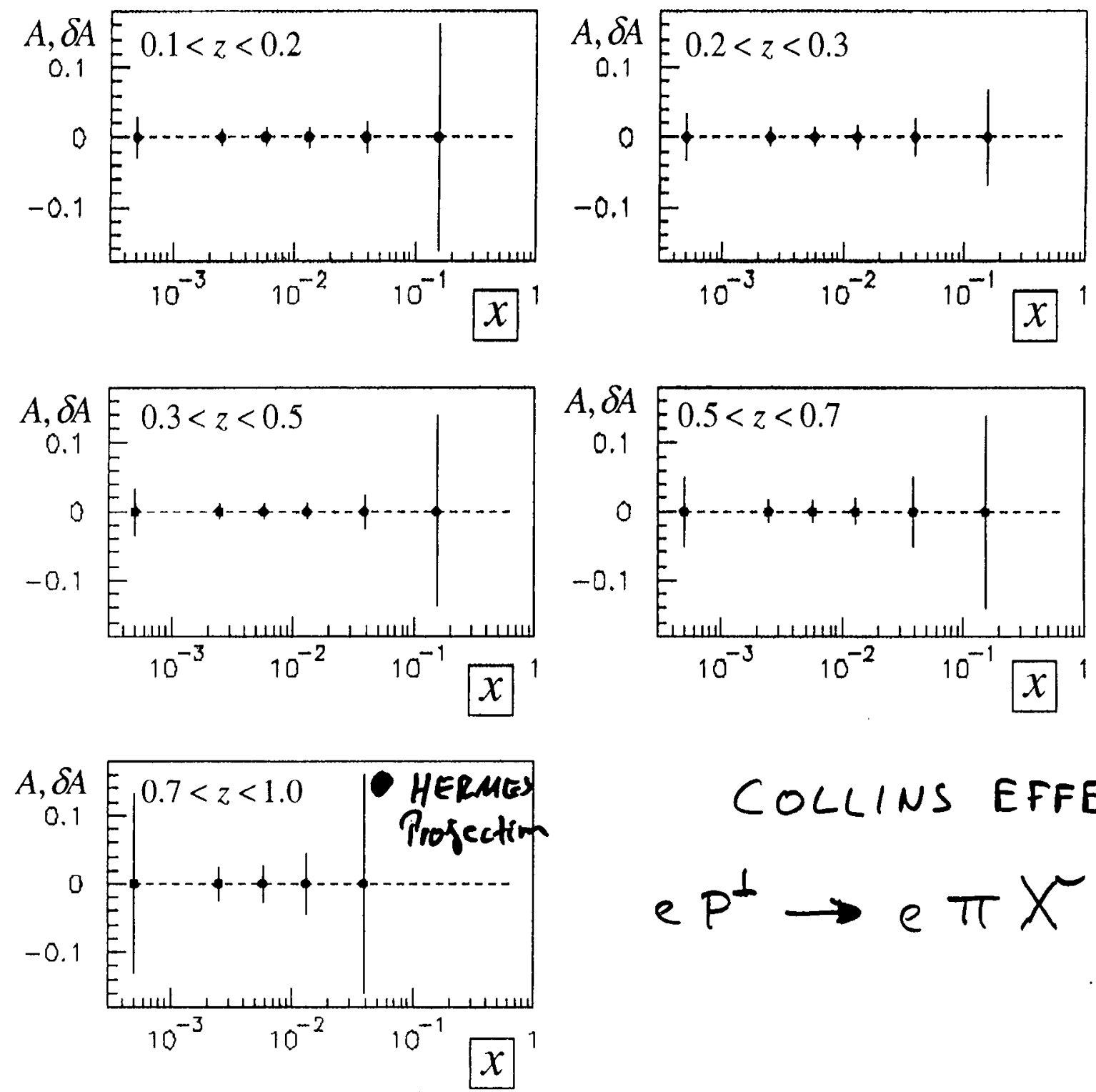

\section{COLLINS EFFECI}

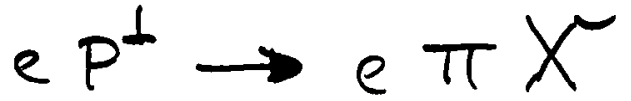




\title{
Role of nuclear shadowing and antishadowing in DIS on polarized nuclear targets
}

\author{
V. Guzey \\ Special Research Centre for the Subatomic Structure of Matter (CSSM), \\ Adelaide University, Australia, 5005
}

\begin{abstract}
We discuss the importance of nuclear shadowing and antishadowing for the interpretation of the experiments on polarized DIS on nuclear targets.
\end{abstract}

\section{INTRODUCTION}

It is well known that nuclear effects play a significant role in unpolarized and polarized deep inelastic scattering (DIS) on nuclear targets. These effects can be divided into coherent and incoherent contributions. Incoherent nuclear effects result from the scattering of the incoming lepton on each individual nucleon, nucleon resonance, or virtual meson of the nucleus, and are present at all Bjorken $x$.

In the target rest frame, coherent nuclear effects arise from the interaction of the incoming lepton with two or more nucleons in the target, and are typically concentrated at low values of Bjorken $x$. Nuclear shadowing at $10^{-5} \div 10^{-4} \leq x \leq 0.05$ and subsequent antishadowing at $0.05 \leq x \leq 0.2$ are examples of coherent effects.

We shall discuss the role played by shadowing and antishadowing in polarized DIS on nuclei. Let us remind that the term "shadowing" refers to the situation when the ratio $R=f_{A} / A f_{N}$ is less than the unity, where $f_{A}$ stands for total inclusive cross sections and/or parton densities in unpolarized DIS and spin asymmetries and/or spin dependent parton densities in polarized DIS. The regime of antishadowing is achieved when $R=f_{A} / A f_{N}>1$.

Nuclear shadowing and antishadowing in unpolarized DIS have been firmly established by experiments, for a recent review see [1]. Nuclear shadowing is present in gluon and quark nuclear parton densities. A recent theoretical analysis of the HERA diffractive data of Ref. [2] demonstrated that nuclear shadowing for the gluon densities is about three times larger than for the quark densities.

While the origin of shadowing - the coherent interaction of the quark-gluon fluctuations of the incoming virtual photon with a few nucleons of the target (in the target rest frame) - is established, the dynamical mechanism of antishadowing is not yet understood. The analysis of the experimental data of Refs. [3-5] suggests that antishadowing is present for the gluon and valence quark parton densities and it is absent for the sea quarks.

We expect that coherent nuclear effects should play a similar, if not larger, role in polarized DIS on nuclear targets. However, they have been ignored in the data analysis so far. We assume that the main reasons for this are large experimental uncertainties and the 
fact that the data were taken on the lightest nuclei - on deuterium by the SLAC E143 [6] and CERN SMC [7] experiments and on ${ }^{3} \mathrm{He}$ by the SLAC E154 [8] and DESY HERMES experiments [9]. Note that all the experiments mentioned above covered the region $x \leq 0.2$, where coherent effects are important.

However, the experimental situation with polarized DIS is changing. Continuous interest in the spin structure of the neutron, proton, and deuteron and recent advances in experimental techniques are leading to more accurate data on various polarized targets. For example, the recent SLAC E155 experiment [10] obtained high precision data on the deuteron spin structure function $g_{1}^{d}\left(x, Q^{2}\right)$ at $0.01 \leq x \leq 0.9$ using a new material, ${ }^{6} \mathrm{LiD}$, as a source of polarized deuterons. We expect that for the ${ }^{6} \mathrm{LiD}$ target nuclear shadowing corrections should be larger than for free deuterons [11].

On the theoretical side, studies of coherent effects in polarized DIS on nuclear targets are very interesting, challenging and promising.

The theoretical analysis of nuclear shadowing on polarized deuterium $[12],{ }^{3} \mathrm{He}[13,14]$ and ${ }^{7} \mathrm{Li}$ [14] revealed that shadowing corrections to the nuclear spin structure function $g_{1}^{A}\left(x, Q^{2}\right)$ are about twice larger than for the unpolarized structure function $F_{2}^{A}\left(x, Q^{2}\right)$ for the corresponding nuclei. Thus, even for the lightest nuclei, D and ${ }^{3} \mathrm{He}$, shadowing corrections are not negligible.

While in the unpolarized DIS the theorem proven by V. Gribov [15] relates in a modelindependent way the leading contribution to nuclear shadowing to the diffractive differential cross section of the process $\gamma^{*}+p \rightarrow X+p^{\prime}$, no such theorem exists for the polarized DIS. Thus, the treatment of shadowing in polarized DIS is a subject of significant theoretical uncertainties. A progress in this directions should provide unique information about the space-time picture of strong interactions involving spin and, in particular, about the interplay between the soft and hard contributions in the wave function of the polarized photon.

On the other hand, the phenomenological treatment of antishadowing in polarized DIS is easier than in unpolarized DIS due to an additional constraint on polarized parton densities because of the Bjorken sum rule [16]. The generalization of the Bjorken sum rule to the trinucleon [13] and ${ }^{7} \mathrm{Li}-{ }^{7} \mathrm{Be}$ systems [14] enables one to model antishadowing - the contributions of shadowing and antishadowing to the first moment of the nuclear non-singlet spin structure function should compensate each other in order to preserve the generalization of Bjorken sum rule, see Eq. (6).

The analysis of Refs. $[13,14]$ demonstrated that nuclear shadowing and antishadowing do affect the extraction of the neutron spin structure function $g_{1}^{n}\left(x, Q^{2}\right)$ from the DIS data on polarized ${ }^{3} \mathrm{He}$. Even larger shadowing corrections are expected to modify the extraction of the deuteron spin structure $g_{1}^{d}\left(x, Q^{2}\right)$ from the ${ }^{6} \mathrm{LiD}$ data [11].

In addition, if we assume by analogy with unpolarized DIS that the shadowing for polarized gluons is about three times larger than for polarized quarks, nuclear shadowing corrections for the polarized gluon density in ${ }^{3} \mathrm{He}$ is of order $30 \%$. This amount of shadowing is enormous for such a light nucleus.

All said above demonstrates that in order to intcrpret corrcctly the results of polarized DIS experiments on nuclear targets one should take into account low Bjorken $x$ coherent phenomena - nuclear shadowing and antishadowing.

Let us outline key steps in the derivation of shadowing and antishadowing contributions in polarized DIS on ${ }^{3} \mathrm{He}[13,14]$ and ${ }^{7} \mathrm{Li}[14]$ and present numerical results. 


\section{SHADOWING AND ANTISHADOWING FOR POLARIZED DIS ON ${ }^{3}$ HE AND ${ }^{7} \mathrm{LI}$}

In the laboratory reference frame, nuclear shadowing is explained by utilizing the spacetime picture of strong interactions. The incoming virtual photon with the high energy $\nu$, momentum $q$ and four-momentum squared $Q^{2}$ interacts strongly with the target via its coherent quark-gluon fluctuations $\left|h_{i}\right\rangle$

$$
\sigma_{\gamma^{*} A}\left(\nu, Q^{2}\right)=\sum_{i}\left|\left\langle\gamma^{*} \mid h_{i}\right\rangle\right|^{2} \sigma_{h_{i} A}\left(\nu, Q^{2}\right),
$$

where $\sigma_{\gamma^{*} A}$ and $\sigma_{h_{i} A}$ are the photon- and $\left|h_{i}\right\rangle$-nucleus cross sections, respectively; $\left|\left\langle\gamma^{*} \mid h_{i}\right\rangle\right|^{2}$ is the probability to find the configuration $\left|h_{i}\right\rangle$ in the photon wave function. The fluctuations $\left|h_{i}\right\rangle$ which contribute to $\sigma_{\gamma^{*} A}$ should live long enough. Their lifetime is also called the coherence length and is defined, using the uncertainty principle, as

$$
l_{c}=\frac{1}{\sqrt{q^{2}+M_{h_{i}}^{2}}-\nu} \simeq \frac{2 q}{M_{h_{i}}^{2}+Q^{2}} \simeq \frac{1}{2 m_{N} x},
$$

where $M_{h_{2}}$ and $m_{N}$ are the masses of the fluctuation $\left|h_{i}\right\rangle$ and nucleon, respectively. In the last step of Eq. (2) was assumed that the dominant contribution to $\sigma_{\gamma^{*} A}$ at a given $Q^{2}$ comes from the quark-gluon fluctuations with $M_{h_{i}}^{2} \approx Q^{2}$.

At small Bjorken $x$, when the coherence length is large,

$$
l_{c}>2 r_{N N}
$$

where $r_{N N} \approx 1.7 \mathrm{fm}$ is the average internucleon distance in the nucleus, the virtual fluctuations $\left|h_{i}\right\rangle$ can interact coherently with two and more nucleons of the target. As well known since the seminal work by Glauber [17], these multiple interactions make $\sigma_{h_{i} A}$ smaller than $A \sigma_{h_{i} N}$, which results in nuclear shadowing for $\sigma_{\gamma^{*} A}$.

In our treatment of nuclear shadowing in Refs. $[13,14]$ we made an approximation to replace the sum in Eq. (1) by an effective fluctuation $\left|h_{e f f}\right\rangle$ with $M_{h_{e f f}}^{2} \approx Q^{2}$ and the scattering $\left|h_{e f f}\right\rangle$-nucleon cross section $\sigma_{e f f}=17 \mathrm{mb}$. In unpolarized DIS, $\sigma_{e f f} \approx 17 \mathrm{mb}$ at $x \approx 10^{-3}$ and $Q^{2} \approx$ few $\mathrm{GeV}^{2}$ has been extracted from the HERA diffractive data in Ref. [2] using the deep connection between shadowing and diffraction, first understood by V. Gribov [15]. Alternatively, a similar value of $\sigma_{e f f}$ can be obtained from an analysis of nuclear shadowing for $A \geq 12$ nuclei. In the case of polarized DIS considered here, we made a hypothesis that the strength of interaction of the effective state $\left|h_{e f f}\right\rangle$ with a nucleon in polarized and unpolarized DIS are similar.

Within this approximation Eq. (1) becomes

$$
\sigma_{\gamma^{*} A}\left(\nu, Q^{2}\right) \propto \sigma_{h_{e f f} A}\left(\nu, Q^{2}\right),
$$

where the $\left|h_{e f f}\right\rangle$-nucleus cross section $\sigma_{h_{e f f} A}\left(\nu, Q^{2}\right)$ can be readily calculated using the Gribov-Glauber multiple scattering formalism $[15,17]$. This formalism requires the knowledge of the nuclear ground-state wave function and the elementary $\left|h_{e f f}\right\rangle$-nucleon scattering amplitude. 
The ground-state wave function for ${ }^{3} \mathrm{He}$ was taken as a pure $S$-wave with a simple Gaussian momentum distribution for the nucleons. This form of the wave function assumes that all spin of ${ }^{3} \mathrm{He}$ is carried by the neutron. The slope of the nuclear form factor, $\alpha=27$ $\mathrm{GeV}^{-2}$, was chosen to reproduce the electromagnetic radius of ${ }^{3} \mathrm{He}$. The $\left|h_{\text {eff }}\right\rangle$-rlucleon scattering amplitude was chosen to be purely imaginary with $B=6 \mathrm{GeV}^{-2}$, where $B$ is the slope of the $\left|h_{e f f}\right\rangle$-nucleon cross section.

Then, the ${ }^{3} \mathrm{He}$ spin structure functions $g_{1}^{3 \mathrm{He}}\left(x, Q^{2}\right)$ is expressed as

$$
\begin{aligned}
& g_{1}^{3} \mathrm{He}\left(x, Q^{2}\right)=\left(1-\frac{\sigma_{e f f}}{4 \pi(\alpha+B)} e^{-\alpha q_{\|}^{2}}+\frac{\sigma_{e f f}^{2}}{48 \pi^{2}(\alpha+B)^{2}} g(x)\right) g_{1}^{n}\left(x, Q^{2}\right)= \\
& \left(1-0.10 e^{-95 x^{2}}+0.004 g(x)\right) g_{1}^{n}\left(x, Q^{2}\right) \text {, }
\end{aligned}
$$

where $q_{\|} \approx 2 m_{N} x$ is the longitudinal momentum transferred to the target; $g(x)$ is a slow function of $x$, normalized as $g(0)=1$. The second and third terms in Eq. (5) correspond to the double and triple scattering contributions to nuclear shadowing, respectively. From Eq. (5) one can see that the shadowing correction is of order $10 \%$ at $x \leq 0.03$. This shadowing correction is twice larger than for unpolarized DIS, where $F_{2}^{3} \mathrm{He}\left(x, Q^{2}\right) / 3 F_{2}^{N}\left(x, Q^{2}\right)=0.95$ in the similar range of $x$.

An important remark here is in order. The $S$-wave type ground-state wave function of ${ }^{3} \mathrm{He}$ is clearly an oversimplification. It well known that higher partial waves, notably $S^{\prime}$ and $P$ waves, are significant in ${ }^{3} \mathrm{He}[18]$. They lead to spin depolarization, which means that the neutron is not $100 \%$ polarized and that the protons also contribute to spin of ${ }^{3} \mathrm{He}$. Calculations of the magnitude of nuclear shadowing with a realistic ground-state wave function of ${ }^{3} \mathrm{He}$ is in progress [19]. Preliminary results seem to indicate that spin depolarization of the nucleons in ${ }^{3} \mathrm{He}$ decreases the amount of nuclear shadowing significantly.

The presence of nuclear shadowing in $g_{1}^{3} \mathrm{He}\left(x, Q^{2}\right)$ violates the relation which follows from the generalization to the tri-nucleon system of the Bjorken sum rule [13]

$$
\eta \equiv \frac{\left.g_{A}\right|_{\text {triton }}}{g_{A}}=\frac{\int_{0}^{1}\left(g_{1}^{{ }^{3} \mathrm{H}}\left(x, Q^{2}\right)-g_{1}^{{ }^{3} \mathrm{He}}\left(x, Q^{2}\right)\right) d x}{\int_{0}^{1}\left(g_{1}^{p}\left(x, Q^{2}\right)-g_{1}^{n}\left(x, Q^{2}\right)\right) d x},
$$

where $\left.g_{A}\right|_{\text {triton }}$ and $g_{A}$ are the axial vector coupling constants measured in the $\beta$ decay of the triton and neutron, respectively. The experimental value of their ratio is $\eta=0.956 \pm 0.004$. Note also that in the approximation that only the neutron is polarized in ${ }^{3} \mathrm{He}$ (only the proton is polarized in $\left.{ }^{3} \mathrm{H}\right)$ the ratios $g_{1}^{3 \mathrm{He}}\left(x, Q^{2}\right) / g_{1}^{n}\left(x, Q^{2}\right)$ and $g_{1 A=3}^{n . s .}\left(x, Q^{2}\right) / g_{1 N}^{n . s .}\left(x, Q^{2}\right)$, the latter is defined as

$$
\frac{g_{1 A=3}^{n . s .}\left(x, Q^{2}\right)}{g_{1 N}^{n . s .}\left(x, Q^{2}\right)} \equiv \frac{g_{1}^{3} \mathrm{He}\left(x, Q^{2}\right)-g_{1}^{3} \mathrm{He}\left(x, Q^{2}\right)}{g_{1}^{n}\left(x, Q^{2}\right)-g_{1}^{p}\left(x, Q^{2}\right)},
$$

are the same.

We suggest to model antishadowing for $g_{1}^{3} \mathrm{He}\left(x, Q^{2}\right)$ at $0.05 \leq x \leq 0.2$ such that its contribution to $\eta$ compensates the effect of shadowing [13]. This criterion fixes the integrated contribution of antishadowing to Eq. (6) but not its shape. In Ref. [14] three scenarios of the shape of antishadowing, depending on the cross-over point $x_{0}$ between the shadowing and antishadowing regions, were considered. The maximum antishadowing (enhancement) of about $40 \% .26 \%$, and $14 \%$ corresponds to $x_{0}=0.1,0.075$, and 0.05 . correspondingly. 
The result of our calculation of nuclear shadowing and modelling of antishadowing (the scenario with largest antishadowing) for the ratio $g_{1 A=3}^{n . s .}\left(x, Q^{2}\right) / g_{1 N}^{n . s .}\left(x, Q^{2}\right)$ is presented in Figure 1. The straight dash-dotted line represents the impulse approximation prediction for $\eta=0.922$, which is known to underestimate the experimental value $\eta=0.956$. This difference can be accounted for by including non-nucleonic degrees of freedom in the ground-state wave function of ${ }^{3} \mathrm{He}-$ the $\Delta(1232)$ isobar $[13,20]$. The curved dotted line is our prediction for $g_{1 A=3}^{\text {n.s. }}\left(x, Q^{2}\right) / g_{1 N}^{\text {n.s. }}\left(x, Q^{2}\right)$ based on Eqs. (5) and (6). Assuming the the discussed effects contribute multiplicatively, we obtain the shape of $g_{1 A=3}^{n . s .}\left(x, Q^{2}\right) / g_{1 N}^{n . s .}\left(x, Q^{2}\right)$, presented as a solid line, which includes shadowing and antishadowing and is in agreement with the experimental value of $\eta$.

One can also use Eq. (5) to estimate the amount of nuclear shadowing in the polarized nuclear gluon parton density $\Delta G_{A}\left(x, Q^{2}\right)$

$$
\frac{\Delta G_{A}\left(x, Q^{2}\right)}{\Delta G_{N}\left(x, Q^{2}\right)}=1-\frac{\sigma_{e f f}}{4 \pi(\alpha+B)} e^{-\alpha q_{\|}^{2}}+\frac{\sigma_{e f f}^{2}}{48 \pi^{2}(\alpha+B)^{2}} g(x),
$$

where $\Delta G_{N}\left(x, Q^{2}\right)$ is the polarized gluon density in a nucleon. Assuming, by analogy with the unpolarized DIS, that $\sigma_{\text {eff }}=3 \times 17=51 \mathrm{mb}$ in the gluon channel, one obtains from Eq. (8) that $\Delta G_{A}\left(x, Q^{2}\right) / \Delta G_{N}\left(x, Q^{2}\right)=0.70$, i.e. the shadowing correction for the polarized gluons in ${ }^{3} \mathrm{He}$ is of order $30 \%$.

While in polarized ${ }^{3} \mathrm{He}$ almost all nuclear polarization is carried by the neutron, in polarized ${ }^{7} \mathrm{Li}$ almost all nuclear spin is provided by the proton. Since the proton spin structure function $g_{1}^{p}\left(x, Q^{2}\right)$ is known with a much better accuracy than $g_{1}^{n}\left(x, Q^{2}\right)$, experimental studies of DIS on polarized ${ }^{7} \mathrm{Li}$ would yield more accurate information about nuclear shadowing in polarized DIS.

Since spin of ${ }^{7} \mathrm{Li}$ is $3 / 2$, there are two spin structure functions $g_{1}^{3 / 23 / 2}\left(x, Q^{2}\right)$ (which we shall call $\left.g_{1}^{7 \mathrm{Li}}\left(x, Q^{2}\right)\right)$ and $g_{1}^{3 / 21 / 2}\left(x, Q^{2}\right)$, corresponding to $M_{J}=3 / 2$ and $1 / 2$, where $M_{J}$ is the $z$ component of the total angular momentum of ${ }^{7} \mathrm{Li}$ [21]. Using the nuclear shell-model ground-state wave function of ${ }^{7} \mathrm{Li}$ along with a simple Gaussian momentum distribution for the nucleons, and employing the Gribov-Glauber scattering formalism, the spin structure function $g_{1}^{7 \mathrm{Li}}\left(x, Q^{2}\right)$ reads [14]

$$
\begin{aligned}
& g_{1}^{7} \mathrm{Li}\left(x, Q^{2}\right)=\frac{13}{15} g_{1}^{p}\left(x, Q^{2}\right)+\frac{2}{15} g_{1}^{n}\left(x, Q^{2}\right) \\
- & \left(0.15 g_{1}^{p}\left(x, Q^{2}\right)+0.03 g_{1}^{n}\left(x, Q^{2}\right)\right) e^{-176 x^{2}}+\left(0.01 g_{1}^{p}\left(x, Q^{2}\right)+0.003 g_{1}^{n}\left(x, Q^{2}\right)\right) g(x) .
\end{aligned}
$$

For the non-singlet combinations of the spin structure functions of ${ }^{7} \mathrm{Li}$ and ${ }^{7} \mathrm{Be}$, defined analogously to Eq. (7), one obtains

$$
\frac{g_{1 A=7}^{n . s}\left(x, Q^{2}\right)}{g_{1 N}^{n . s}\left(x, Q^{2}\right)}=\frac{11}{15}\left(1-0.18 e^{-176 x^{2}}+0.02 g(x)\right) .
$$

Eq. (10) predicts nuclear shadowing corrections of order $16 \%$. This is by about a factor of two larger than the shadowing corrections for the ratio $F_{2}^{7 \mathrm{Li}}\left(x, Q^{2}\right) / 7 F_{2}^{N}\left(x, Q^{2}\right)$ [14].

Similarly to the case of ${ }^{3} \mathrm{He}$ considered above, the significant shadowing for the ratio $g_{1 . A=7}^{n . s}\left(x, Q^{2}\right) / g_{1 . N}^{n . s}\left(x, Q^{2}\right)$ must be compensated by some antishadowing in order to preserve the generalization of the Bjorken sum rule for the ${ }^{7} \mathrm{Li}^{7} \mathrm{Be}$ system, see Fi. (6). In Figure 2 
we present our predictions for the ratio $15 / 11 g_{1 A=7}^{n . s}\left(x, Q^{2}\right) / g_{1 N}^{n . s}\left(x, Q^{2}\right)$ as a function of $x$. In the absence of the shadowing and antishadowing effects, this ratio would be the unity. The three solid lines correspond to three different positions of the cross-over point $x_{0}$. One can see that, depending on $x_{0}$, the effect of antishadowing could be as large as 20 (55)\%.

\section{CONCLUSIONS}

We demonstrated that such coherent nuclear effects as shadowing and antishadowing play a significant role in polarized DIS on nuclear targets of ${ }^{3} \mathrm{He}$ and ${ }^{7} \mathrm{Li}$. Therefore, these effects should be taken into account in order to correctly interpret the results of experiments on polarized DIS on nuclei. In particular, the extraction of the neutron spin structure function $g_{1}^{n}\left(x, Q^{2}\right)$ from the ${ }^{3} \mathrm{He}$ data is affected by shadowing and antishadowing.

While shadowing and antishadowing corrections to the spin structure functions are still within the present experimental uncertainties, in the future, more accurate experimental data and the use of various (heavier) polarized nuclear targets will allow to firmly establish the importance of nuclear shadowing and antishadowing in polarized DIS. This will boost further theoretical interest in the understanding of the dynamics of shadowing and, maybe, antishadowing, in terms of quark and gluon fluctuations of the wave function of the polarized photon. 


\section{REFERENCES}

[1] G. Piller and W. Weise, Phys. Rep. 330, 1 (2000).

[2] L. Frankfurt and M. Strikman, Eur. Phys. J. A 5 (1999) 293.

[3] L. Frankfurt and M. Strikman, Phys. Rep. 160, 235 (1988).

[4] L. Frankfurt, M. Strikman, and S. Liuti, Phys. Rev. Lett. 65, 1725 (1990).

[5] T. Gousset and H.J. Pirner, Phys. Lett. B 375, 349 (1996).

[6] E143 Collab., K. Abe et al., Phys. Rev. D 58, 112003 (1998).

[7] SMC Collab., B. Adeva et al., Phys. Rev. D 58, 112001 (1998).

[8] E154 Collab., K. Abe et al., Phys. Lett. B 405, 180 (1997); K. Abe et al., Phys. Rev. Lett. 79, 26 (1997)

[9] HERMES Collab., K. Ackerstaff et al., Phys. Lett. B 404, 383 (1997).

[10] E155 Collab., P.L. Anthony et al., preprint hep-ex/9904002, accepted for publication in Phys. Lett. B.

[11] V. Guzey, in preparation.

[12] J. Edelmann, G. Piller, and W. Weise, Z. Phys. A 357, 129 (1997); J. Edelmann, G. Piller, and W. Weise, Phys. Rev. C 57, 3392 (1998).

[13] L. Frankfurt, V. Guzey, and M. Strikman, Phys. Lett. B 381, 379 (1996).

[14] V. Guzey and M. Strikman, Phys. Rev. C 61, 014002, (2000).

[15] V.N. Gribov, Sov. J. Nucl. Phys. 9, 369 (1969); Sov. Phys. JETP 29, 483 (1969); ibid 30, 709 (1970).

[16] J.D. Bjorken, Phys. Rev. 148, 1467, (1966).

[17] R.J. Glauber, Phys. Rev. 100, 1696 (1978).

[18] B. Blankleider and R.M. Woloshyn, Phys. Rev. C 29, 538 (1984).

[19] F. Bissey, V. Guzey, M. Strikman, and A.W. Thomas, in preparation.

[20] C. Boros, V. Guzey, M. Strikman, and A.W. Thomas, preprint hep-ph/0008064.

[21] R.L. Jaffe and A. Manohar, Nucl. Phys. B 321, 343 (1989). 


\section{FIGURES}

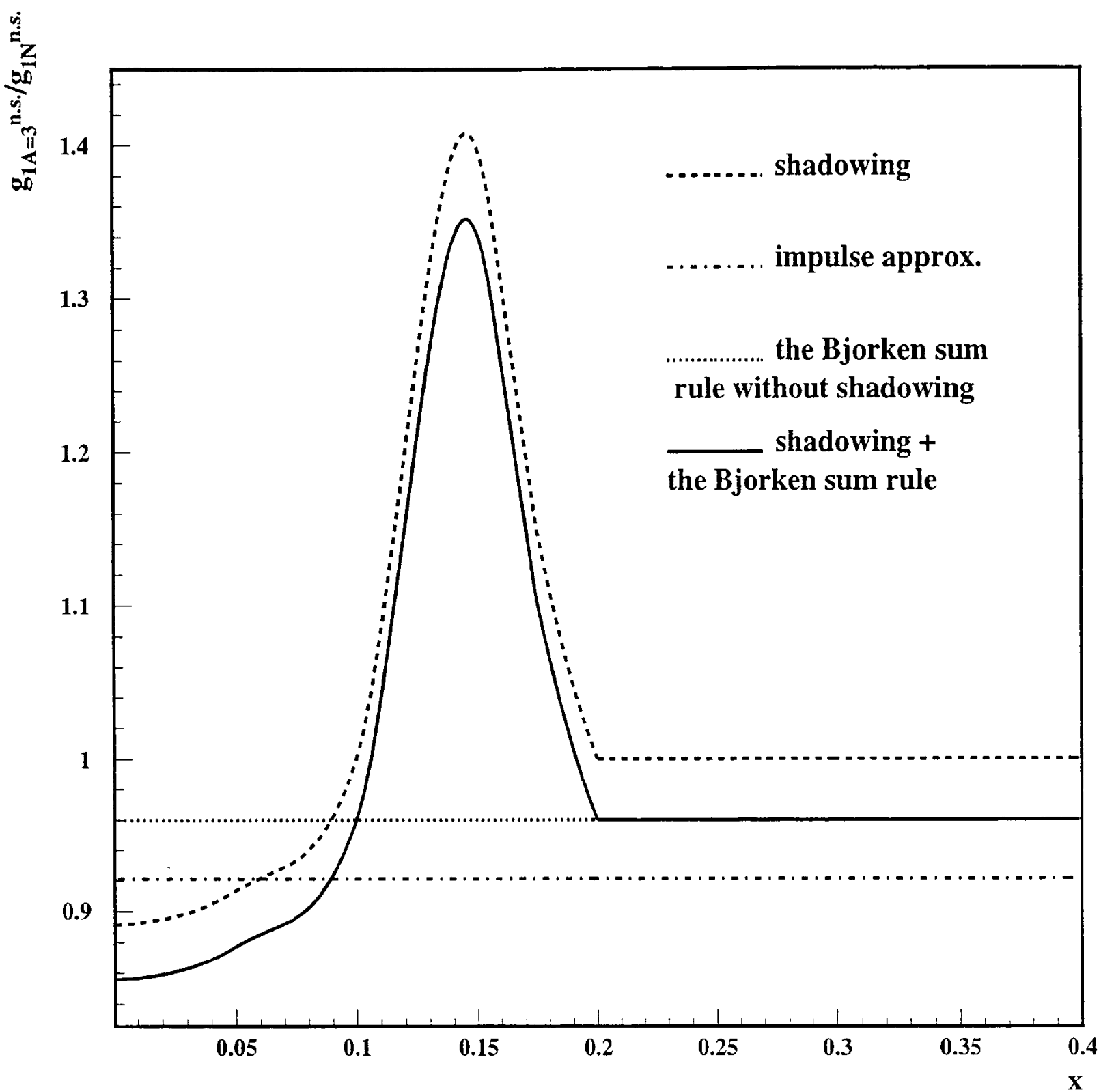

FIG. 1. $g_{1 A=3}^{\text {n.s. }}\left(x, Q^{2}\right) / g_{1 N}^{\text {n.s. }}\left(x, Q^{2}\right)$ as a function of $x$. The dashed curve represents the calculation of nuclear shadowing given by Eq. (5) at $x \leq 0.05$ and the modelling of antishadowing at $0.05 \leq x \leq 0.2$ to preserve the ratio of $\mathrm{Eq}$. (6). The two straight lines correspond to $\eta=0.956$ (upper) and $\eta=0.922$ (lower), which are the predictions for $g_{1 A=3}^{n . s}\left(x, Q^{2}\right) / g_{1 N}^{n s}\left(x, Q^{2}\right)$, when the shadowing and antishadowing effects are neglected. The solid line is our prediction for $g_{1 A=3}^{n . s .}\left(x, Q^{2}\right) / g_{1 N}^{n . s .}\left(x, Q^{2}\right)$, which corresponds to the experimental value of $\eta=0.956$ in Eq. (6). 


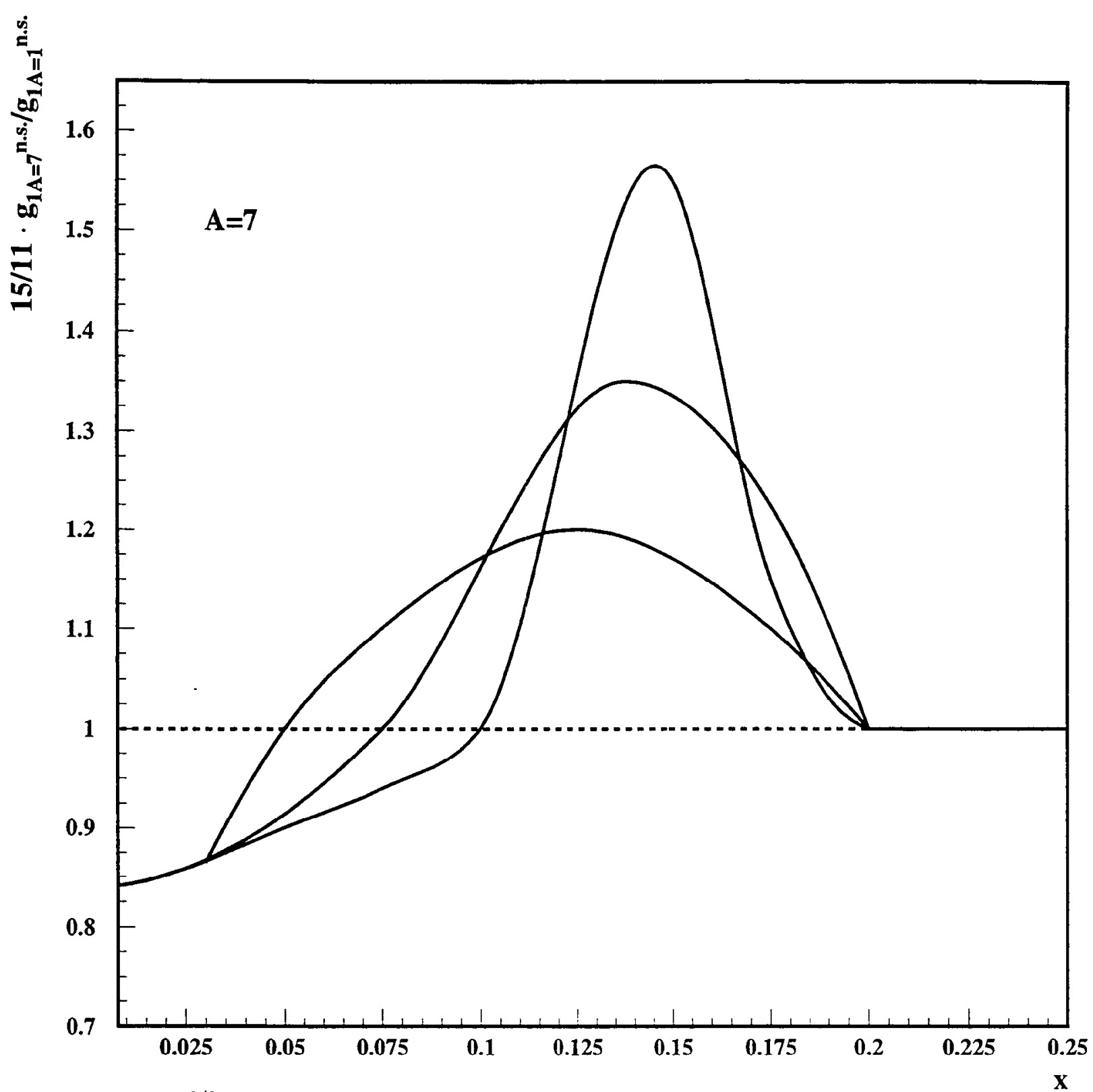

FIG. 2. $g_{1 A=7}^{\text {n.s. } 3 / 2}\left(x, Q^{2}\right) / g_{1 N}^{\text {n.s. }}\left(x, Q^{2}\right)$ as a function of $x$. The solid curves are the result of our calculation of shadowing of Eq. (10) at $x \leq 0.05$ and modelling of antishadowing at $0.05 \leq x \leq 0.2$ to preserve the ratio of Eq. (6). The three curves correspond to three scenarios of the shape of antishadowing with the cross-over points between the shadowing and antishadowing regions at $x_{0}=0.1,0.075$, and 0.05 . 
TAUP-2643-2000

September 20, 2000

\title{
A manifestation of a gluon saturation in e-A DIS
}

\author{
Eugene Levin ${ }^{1,2}$ and Uri Maor ${ }^{1}$ \\ 1 HEP Department, School of Physics and Astronomy \\ Tel Aviv University, Tel Aviv 69978, Israel \\ 2 Desy Theory, 22603 Hanburg, Germany
}

\begin{abstract}
This is a short presentation of our talks given at eRHIC Workshop at the BNL. We give here a status report of our attempts to understand how gluon saturation will manifest itself in deep inelastic scattering with nuclei. This summary reflects our current understanding and shows directions of our research rather then a final answer to the question. Nevertheless, we are able to share with our reader our tentative answer to the question: "Why do we need to measure DIS with nuclei and why these data will be complementary to the information obtained from proton DIS".
\end{abstract}

\section{INTRODUCTION: WHAT ARE THE SCALES IN PHOTON-NUCLEUS DIS?}

The main goal of these notes is to examine if and how we can observe the phenomenon of gluon saturation in DIS with nucleus. We present here only a status report of our attempts to clarify this subject, which is far away from being complete. It, rather, indicates the directions of our searches. Much more work is needed to develop a reliable approach so as to finalize our recommendations concerning experiments the most sensitive to the gluon saturation.

We start with the general approach to photon-nucleus interaction, developed by Gribov [1] who suggested following time sequence of this process:

1. First, the $\gamma^{*}$ fluctuates into a hadron ( quark - antiquark) system well before the interaction with the target;

2. Then the converted quark-antiquark pair (or hadron system) interacts with the target.

Generally, these two stages result in the following formula for the cross section

$$
\sigma_{t o t}\left(\gamma^{*}+A\right)=\sum_{n}\left|\Psi_{n}\right|^{2} \sigma_{t o t}(n+A ; x)
$$

where $\Psi_{n}$ is the wave function of the system, produced in the first stage of the process.

\section{A. Separation scale $\mathbf{r}_{\perp}^{\text {sep }} \approx 1 / \mathrm{M}_{0}$}

This scale is a typical distance which separates the PQCD approach from the non-perturbative one. Roughly speaking, for shorter distances than $r_{\perp}^{\text {sep }}$, the QCD running coupling constant can be considered as a small parameter while for longer distances $\alpha_{S}\left(r_{1}\right)$ is large and we cannot use the powerful methods of pQCD. Table 1 demonstrates how this scale works in our particular model to incorporate the long distance physics $[2-4]$. 
Table 1

\begin{tabular}{lll}
\hline \hline Perturbative QCD & $\longrightarrow$ & non-perturbative QCD \\
short distances & $\rightarrow$ & long distances \\
$r_{\perp}<$ & $r_{\perp}^{\text {sep }}$ & $<r_{\perp}$ \\
DOF: colour dipoles [5] & - & DOF: constituent quarks [6] \\
$\Psi_{n}:$ QED for virtual photon & - & $\Psi_{n}:$ generalized VDM for hadronic system \\
$\sigma_{\text {tor }}(n, x)=\sigma\left(r_{t}^{2}, x\right)$ & - & $\sigma_{\text {tot }}(n, x)=\sigma(q q \rightarrow q q ; x)$ \\
Glauber- Mueller Eikonal $[7]$ for $\sigma\left(r_{\perp}^{2}, x\right)$ & - & Regge phenomenology for $\sigma(q+q \rightarrow q+q ; x)$ \\
\hline \hline
\end{tabular}

It is important to notice that the separation scale mostly relates to the produced hadronic $(q \bar{q}))$ system and does not depend on the properties of the target (in particular, the atomic number ). From Table 1 one can write for short distances $\left(r_{\perp}<r_{\perp}^{s e p}\right)$

$$
\sigma_{t o t}\left(\gamma^{*} p\right)=\int d^{2} r_{\perp} \int_{0}^{1} d z\left|\Psi\left(Q^{2} ; r_{\perp}, z\right)\right|^{2} \sigma_{t o t}\left(r_{\perp}^{2}, x\right)
$$

\section{B. Saturation scale $r_{\perp}^{\text {sat }} \approx 1 / Q_{s}(x ; A)$}

At low $x$ and at $r_{\perp}<r_{\perp}^{s e p}$ we believe [8] that The system of partons always passes the stage of hdQCD. (at shorter distances) before it goes to the black box, which we call non-perturbative $Q C D$, and which, in practice, we describe in old fashion Reggeon phenomenology. At the hdQCD stage we have to observe a parton system with sufficiently small typical distances $\left(r_{\perp}^{s a t} \approx 1 / Q_{s}(x ; A)\right)$ at which the QCD coupling constant is still small $\left(\alpha_{S}\left(r_{\perp}^{s a t}\right) \ll 1\right)$, but the density of partons is so large that we cannot use the pQCD methods in our calculations. The picture of the parton distribution in the transverse plane is shown in Fig. 1 .

The estimate of the value for the saturation scale is obtained [8] from the equation

$$
\kappa=\frac{3 \pi^{2} \alpha_{S} A}{2 Q_{s}^{2}(x)} \times \frac{x G\left(x, Q_{s}^{2}(x)\right)}{\pi R_{A}^{2}}=1
$$

where $A$ and $R_{A}$ are the atomic number and radius of the nucleus. Eq. (3) has a simple physical meaning giving the probability of the interaction between two partons in the parton cascade. Namely, such an interaction will stop the increase of the parton density due to parton emission, which is included in the DGLAP evolution equations [9]

It is important to notice that the saturation scale strongly depends on $A\left(Q_{s}(x ; A) \propto A^{\frac{1}{6}}[10] \div A^{\frac{1}{3}}[11]\right)$.

\section{The theory status}

In eA deep inelastic scattering we want to find the high density parton system which is a non-perturbative system but which can be treated theoretically. It should be stressed that the theory of hdQCD is in a very good shape now. Two approaches have been developed for hdQCD: the first one [12] is based on PQCD ( see GLR and Mueller and Qiu in Ref. [8]) and on the dipole degrees of freedom [5], while the second [13] uses the effective Lagrangian, suggested by McLerran and Venugopalan [8]. As a result of this intensive work we know now the nonlinear equation which governs the QCD evolution in the hdQCD region [14]. We have not developed simple methods to estimate an effect of hdQCD on the experimental observables and have to use a model approximation, but we want to emphasize that this is a temporary stage of our theory which will be overcome soon. 


\section{HERA: RESULTS AND PUZZLES.}

We start answering the question: "why do we need a nuclear target to find the hdQCD phase" with a summary of what we have learned from HERA.

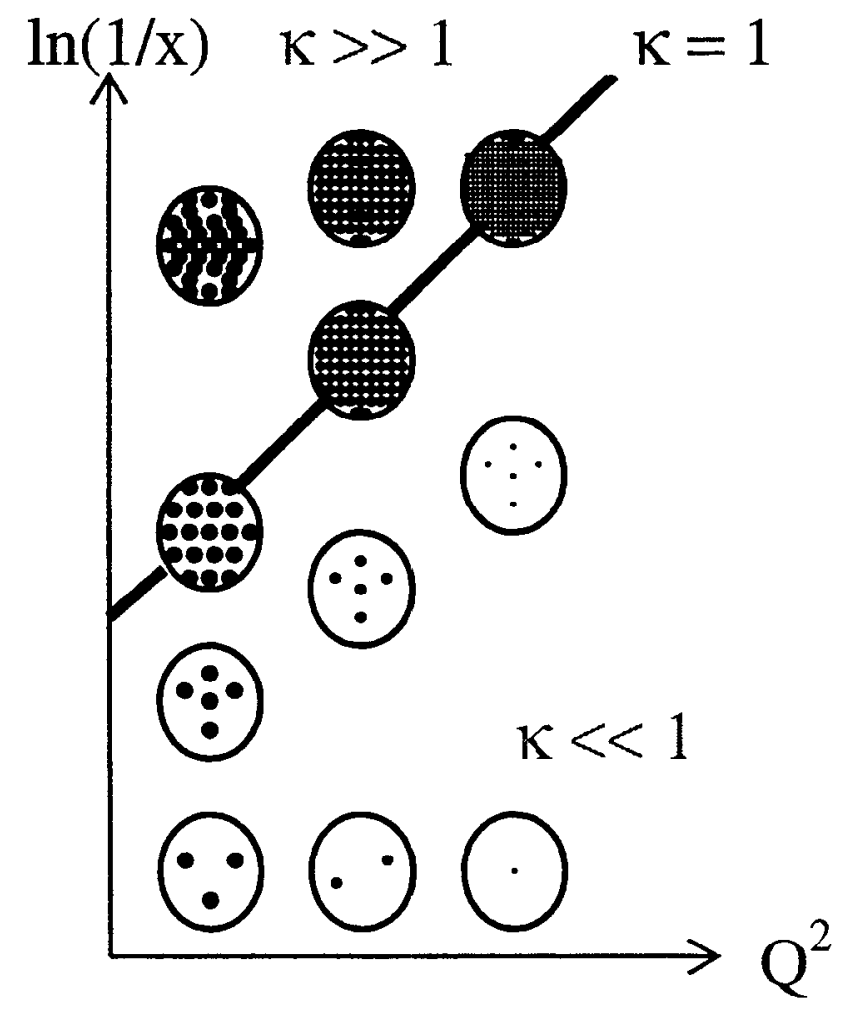

FIG. 1. The parton distribution in the transverse plane. The curve shows the saturation scale $Q_{s}(x ; A)$

- $\mathbf{F}_{2}$ - the most striking and significant result from HERA is the increase of $F_{2}$ at low $x$ [15]. The interpretation of the $F_{2}$ data in terms of the DGLAP evolution equations leads to sufficient large value and a steep behaviour of the gluon structure function at low $x . x G\left(x, Q^{2}\right)$ turns out to be so large that $\kappa$, our new order parameter, exceeds unity in a significant part of the accessible phase space (see Fig. 2).

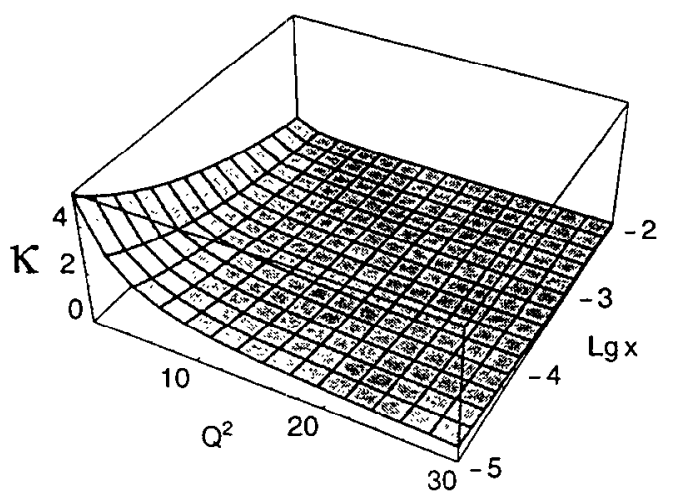

FIG. 2. 
- Diffractive production - three important results have been observed at HERA: (i) the diffractive production gives a substantial part of the total cross section, about $10 \div 15 \%$ at $Q^{2} \approx 10 \mathrm{GeV}^{2}$; (ii) the energy behaviour of the diffractive cross section has an intercept larger that the intercept of the soft Pomeron, namely, $\sigma_{d i f f} \propto(1 / x)^{2 \Delta_{P}}$ with $\Delta_{P}>\Delta_{\text {soft } P} \approx 0.1[16]$, and (iii) the ratio $\sigma_{\text {diff }} / \sigma_{\text {tot }}$ is a constant versus energy in HERA kinematic region. From Fig. 1, one can see that a hadron looks as a diffractive grid with typical size of the order of $r^{\text {sat }}$. Therefore, we expect that diffractive processes originate from a rather small distances. This fact leads to a natural explanation of the energy behaviour of the diffractive cross section.

- Matching between soft and hard processes. The experimental data on $\gamma^{*} p$ cross section at small $Q^{2}$ allows to test different models for the matching of the soft and hard interactions.

- The dedicated beautiful measurement of the $F_{2}$ slope $\left(d F_{2} / \ln Q^{2}\right)$ gives us a hope to find the saturation scale by observing the movement of the maxima in $Q^{2}$ - behaviour at fixed $x$. The experimental data show a considerable deviation fron the DGLAP analysis at $Q^{2} \leq 1 \div 3 \mathrm{GeV}^{2}$. However, the current data can be described in two different ways, either due to a gluon saturation or due to a probable matching between soft and hard at rather large momenta ( about $1-2 \mathrm{GeV}$ ) [4]. It should be noticed, however, that the $J / \Psi$ production can be easily described taking into account shadowing corrections confirming a gluon saturation hypothesis [4].

We listed above the most important HERA observations which indicate a possible saturation effect. To illustrate this fact we will demonstrate that a simple parameterization of Golec-Biernat and Wusthoff [17]. which includes the saturation, works well. They found an elegant phenomenological model for $\sigma\left(r_{\perp}^{2}, x\right)$ in Eq. (2) which is able to describe all experimental data using only three parameters [17]. In this model

$$
\sigma_{\text {dipole }}\left(r_{\perp}, x\right)=\sigma_{0}\left(1-\exp \frac{-\frac{r_{1}^{2} Q_{0}^{2}}{\left(x / x_{0}\right)^{\lambda}}}{2 .}\right.
$$

with $\sigma_{0}=23.03 \mathrm{mb}, Q_{0}^{2}=1 \mathrm{GeV}^{2}, x_{0}=0.0003$ and $\lambda=0.288$. Figs. 3 and 4 show the quality of this description.

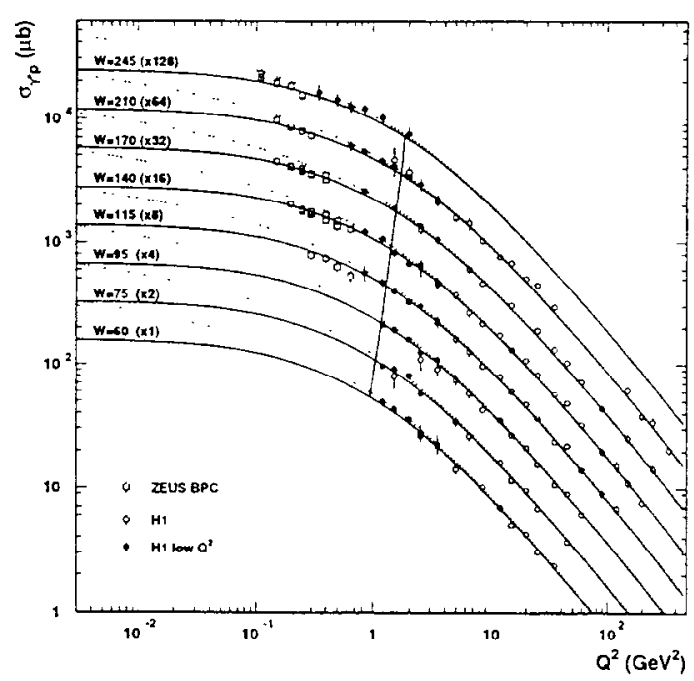

FIG. 3 
Note that even though the above description is impressive, it cannot fix the value of the saturation scale from the data which is too constrained by the kinematics ( see Ref. [4] for details).

Thefore, we can conclude that the saturation hypothesis is compatible with all experimental data. However, the puzzling situation is that the same data can be described fron a different point of view without a saturation scale in the standart DGLAP evolution equation for $Q^{2}>1 \mathrm{GeV}^{2}$ and the soft phenomenology for $Q^{2}<1 G e V^{2}$. We do not claim that it is a reasonable or smooth parameterization of the data but Donachie-Landshoff mixture of soft and hard Pomeron shows that we can produce such a model.

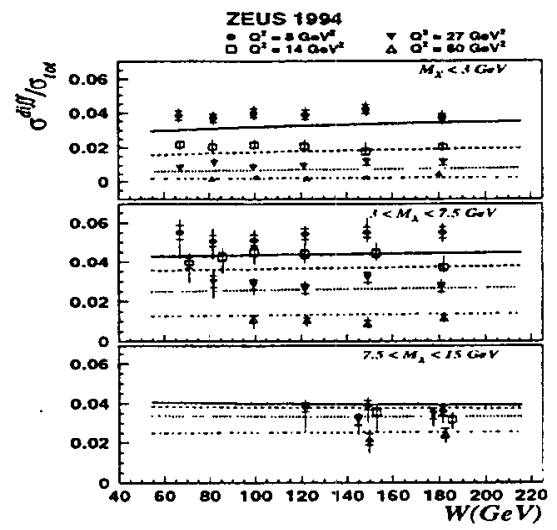

FIG. 4 .

Thus in order to fix the saturation scale and to descriminate between competing models, we need either to reach a much smaller values of $x$ ( higher energy) or to use a new target. Realistically, we can conclude that

We need DIS with nuclei to check whether the indications on saturation effect at HERA are really true.

\section{SCALE OF THE SATURATION EFFECT FOR DIS WITH NUCLEI}

\section{A. Asymptotic predictions.}

Let us start with listing the asymptotic predictions of our approach [2-4] which is based on the GlauberMueller formula for $\sigma\left(r_{\perp}^{2}, x\right)$ in Eq. (2) [7].

- At fixed $r_{\perp}$ and at $x \rightarrow 0$

$$
\sigma_{\text {tot }}^{\text {dipole }} \longrightarrow 2 \pi\left(R_{A}+\frac{h}{2} \ln \left(Q_{s}^{2}(x ; A) / Q^{2}\right)\right)
$$

where $R_{A}$ is the nucleus radius and $h$ is the surface thickness in the Wood-Saxon nucleon density :

- In the same limit $\frac{d F_{2}^{A}}{d \ln Q^{2}} \longrightarrow F_{2}^{A}\left(1-\frac{h}{R_{A}}\right) \propto Q^{2} R_{A}^{2}$;

- The ratio of the diffraction to the total cross sections should depend on energy only weakly [18];

$$
\frac{\sigma_{\text {tot }}^{\text {diffaction }}\left(\gamma^{*} A\right)}{\sigma_{\text {tot }}\left(\gamma^{*} A\right)} \approx \operatorname{Const}(W) \longrightarrow(\text { slowly }) \frac{1}{2}
$$

- The energy behaviour of $\sigma^{\text {diffraction }}\left(\gamma^{*} A\right)$ is determined by short distances $r_{\perp} \approx 1 / Q_{s}(x ; A)$ :

- The high density effects should be stronger in the diffractive chamels. 


\section{B. $x G_{A}\left(x, Q^{2}\right)$}

In Fig. 5 we present our calculation of the gluon structure function for different nuclei. Fig.5-d gives a glimpse at what we are taking into account in our approach. Figs. 5-a - 5-c show the comparison of our calculations, based on the Glauber-Mueller formula, with the solution of the full equation for hdQCD [14] ${ }^{1}$

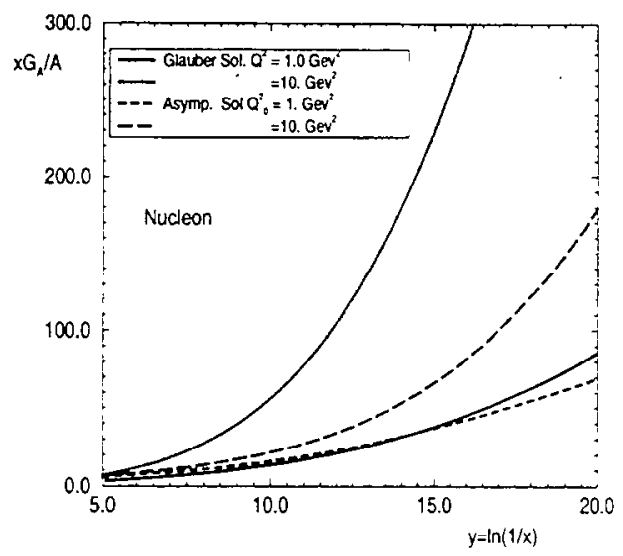

Fig. 5-a.

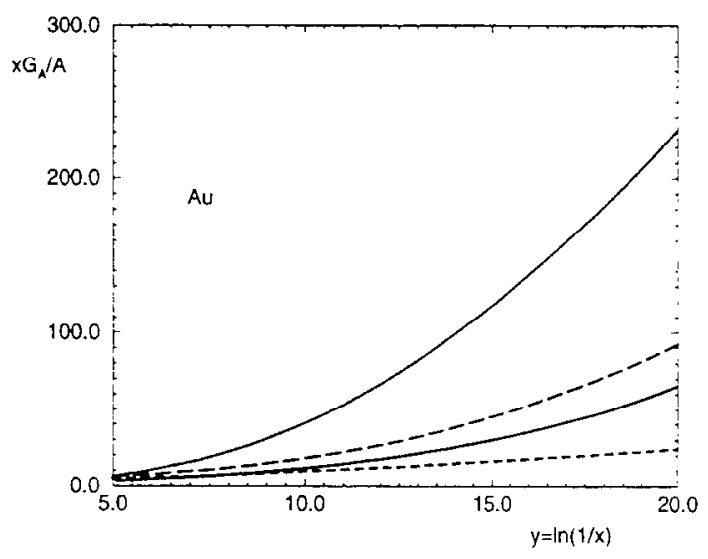

Fig. 5-c

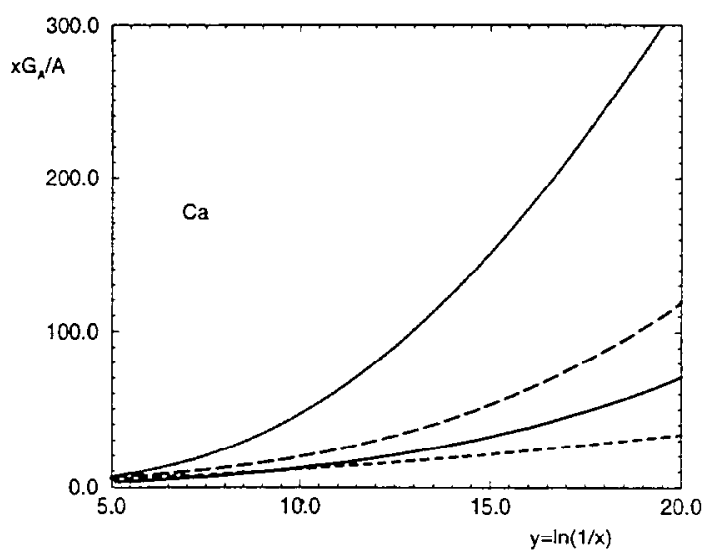

Fig. 5 -b

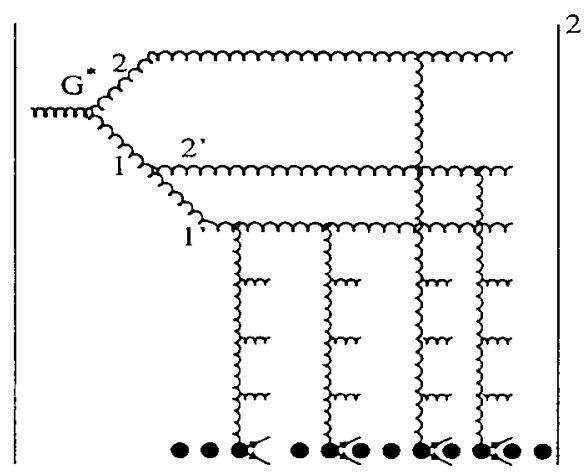

Fig.5-d

FIG. 5 .

We can derive two conclusions from Fig.5: (i) the saturation effect is much stronger for a nucler target than for a nucleon, and (ii) our model underestimates the value of the effect for $Q^{2} \approx 1 \mathrm{GeV}^{2}$. Unfortunately, we have not finished our estimates for $F_{2}$ for DIS with nuclei.

\footnotetext{
'Actually, the equation, suggested in Ref. [3] was solved and plotted in Fig. 5 as the asymptotic solution. but this equation in the double $\log$ approximation coincides with the correct one [14].
} 


$$
\text { C. } R=\frac{\sigma_{\text {tot }}^{\text {diffretion }}}{\sigma_{\text {tot }}}
$$

This ratio shows us how we are close, or how we are far away, from the asymptotic regime since at very high energy it should be equal to $\frac{1}{2}$. In Fig. 6 we plotted our calculations for this ratio [19]. One can see that the ratio is larger than for the proton target ( see Fig, 4 ) but it is still smaller than the limiting value of $\frac{1}{2}$. This is a very encouraging fact for experiment since we do not want to measure a black disc limit which is not sensitive to the theoretical approach. In other words, any model or any theoretical approach will give the unitarity limit which we call "black disck limit". A ll our theoretical QCD prediction are related to the form of the transition from PQCD to the "black disc limit".

$A=30$
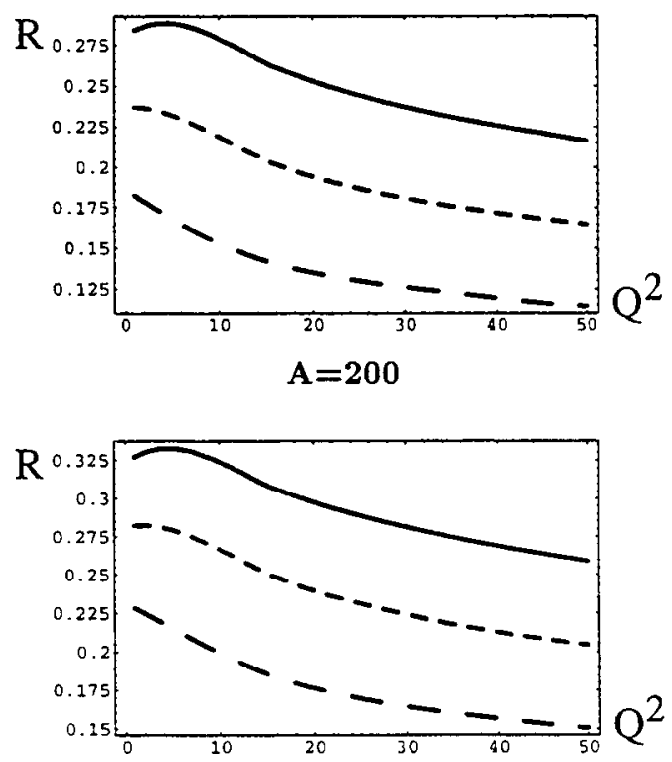

$A=100$
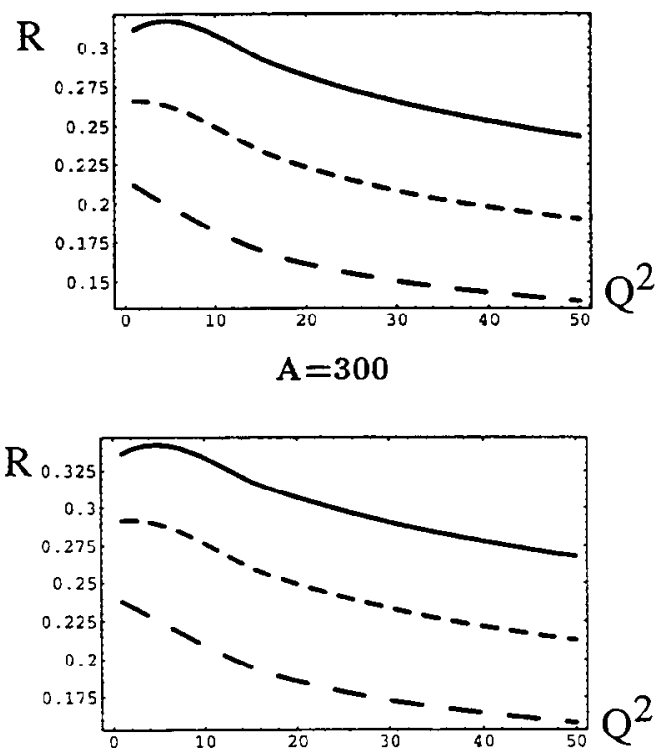

FIG. 6.

D. $F_{2}$ slope $\left(\frac{\partial F_{2}^{A}\left(x, Q^{2}\right.}{\partial \ln Q^{2}}\right)$

As we have mentioned, gluon satruration leads to a maximum in the $Q^{2}$ dependence of $F_{2}$ slope at $Q^{2}=Q_{s}^{2}(x ; A)$ fora fixed value of $x$. Such a maximum has not been seen in the HERA data and has not been anticipated in our estimates of the slope. However, the numerical value of the gluon saturation is rather for a nucleus target. Figs. 7 and 8 display the possible experimental effect. In these figures the value of the damping factor $\left(D^{A}\right)$ for the $F_{2}$ slope is plotted. $D^{A}$ is defined in the following way:

$$
\frac{d F_{2}^{A}\left(x, Q^{2}\right)}{d \ln Q^{2}}=D^{A}\left(x, Q^{2}\right) A \frac{d F_{2}^{N ; D G L A P}\left(x, Q^{2}\right)}{d \ln Q^{2}},
$$

where $F_{2}^{N ; D G L A P}$ is the $F_{2}$ sructure function for a nucleon in the DGLAP approximation. It turns out that the value of the effect is sizable for $Q^{2}<10 \mathrm{GeV}^{2}$ and strongly depends on $A$. 

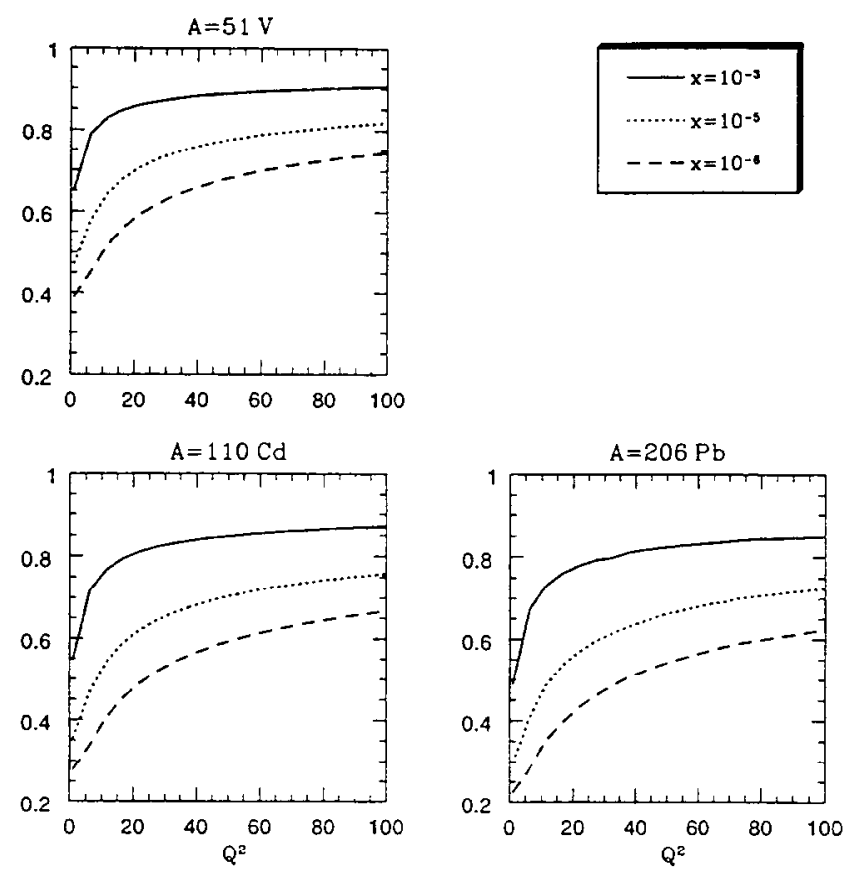

FIG. 7.
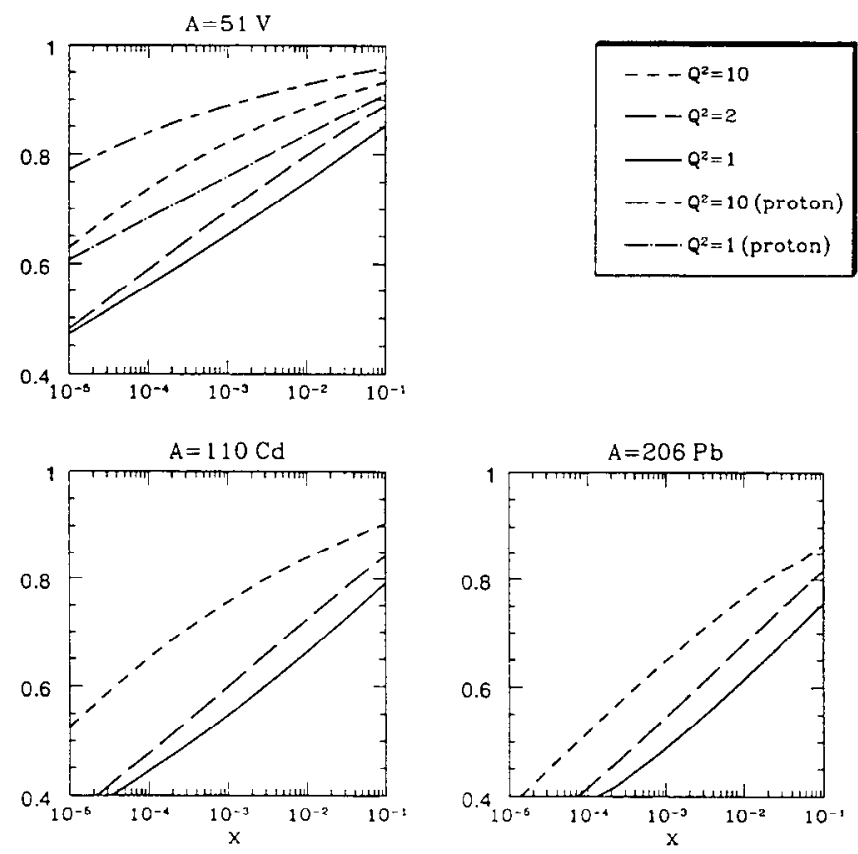

FIG 8. 

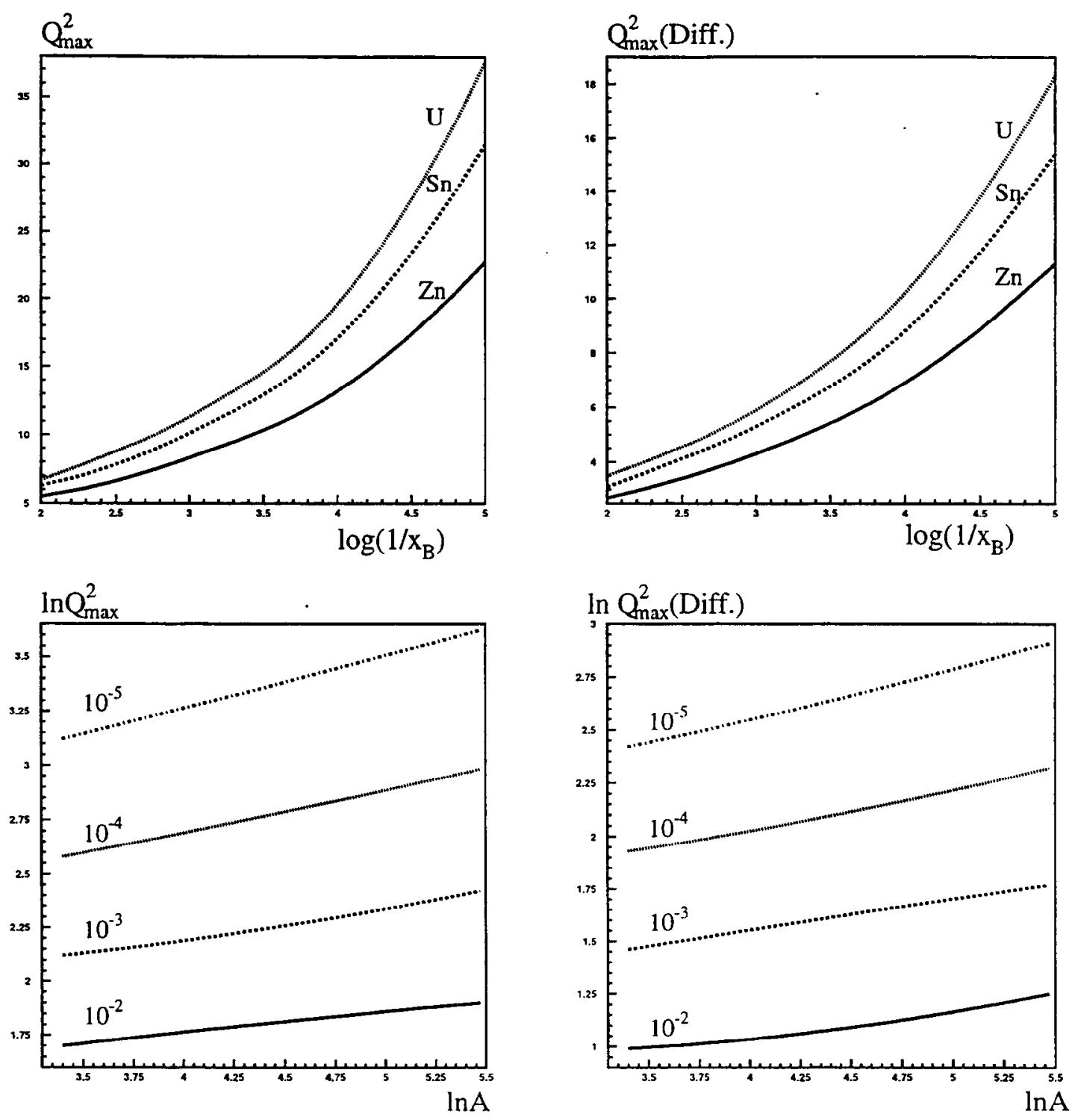

FIG. 10.

B. Higher twists in $F_{L, T}$ and in $F_{L, T}^{D}$.

It is well known that any structure function $\left(F_{2}\left(x, Q^{2}\right)\right.$ for example $)$ can be written

$$
F_{2}\left(x, Q^{2}\right)=F_{2}^{L T}\left(x, \ln Q^{2}\right)+\frac{M^{2}}{Q^{2}} F_{2}^{H T}\left(x, \ln Q^{2}\right)+\ldots+\left(\frac{M^{2}}{Q^{2}}\right)^{n} F_{2}^{n T}\left(x, \ln Q^{2}\right)+\ldots
$$

Terms, which are small in terms of $Q^{2}$ power, called higher twist contributions. The $\ln Q^{2}$ dependence of the leading and higher twist structure functions ( $F_{2}^{L T}$ and $F_{2}^{H T}$ in Eq. (6) ) is governed by the evolution equations. The DGLAP evolution equations [9] give the $\ln Q^{2}$ dependence of the leading twist structure function $\left(F_{2}^{L T}\right)$ only. Unfortunately, we know only a little about the higher twist contributions. 
1. We know the evolution equations for all higher twist structure functions [21];

2. We know the behaviour of the higher twist structure functions at low $x$ [22]. For example,

$$
\left.F_{2}^{H T}\left(x, \ln Q^{2}\right)\right|_{x \ll 1} \longrightarrow F_{2}^{L T}\left(x, \ln Q^{2}\right) \cdot x G^{L T}\left(x, \ln Q^{2}\right) ;
$$

3. We know, that higher twist contributions are needed to describe the experimental data [23].

However, it is difficult to estimate the value of the higher twist contributions. Following Ref. [24], we estimate the value of different twist contribution for e A scattering.

Fig.11 shows that the higher twist contributions for nucleus target become smaller than the leading twist one only at $Q^{2}>5 \mathrm{GeV}^{2}$ even at $x=10^{-2}$. It gives us a hope to treat them theoretically.

\section{CONCLUSIONS.}

1. We have a solid theoretical approach for eA DIS, but we need more experience in numerical solution of the non-linear equation specifically for eRHIC kinematic region;

2. We know pretty well the scale of SC for eA interaction, but we need more systematic study of DGLAP evolution for nuclear structure functions and a special investigation whether the initial parton distributions could be calculated for nuclear target from the initial parton distributions for proton ;

3. Our estimates show that we will be able to see the saturation scale in eA DIS at eRHIC being still far away from trivial blackening of high energy interaction with nuclei, but we need to check how close our model, which we use inn practise, to theoretical estimates;

4. The $F_{2}^{A}$-slope is a very sensitive observable for the saturation scale, but, unfortunaly, we cannot expect a qualitatively different behaviou for the saturation models in comparison with others:

5. Maxima in ratios of $F_{L} / F_{T}$ and $F_{L}^{D} / F_{T}^{D}$ give promising tool to extract the value of saturation scale $Q_{s}(x ; A)$, but we need more study on this subject and, in particular, how the initial parton distribution for DGLAP evolution could affect our predictions;

6. e $\Lambda$ DIS is very instructive for separation of leading and higher twist contributions, since the fact that typical momentum at which these two contributions become of the same order is growing with $A$.

\section{ACKNOWLEDGMENTS}

The authors are very much indebted to our coauthors Errol Gotsman, Larry McLerran, Eran Naftali and Kirill Tuchin for their help and everyday discussions on the subject. E. L. thanks BNL Nuclear Theory group and DESY Theory group for their hospitality and creative atmosphere during several stages of this work

This research was supported in part by the BSF grant \# 9800276 and by Israeli Science Foundation, founded by the Israeli Academy of Science and Humanities. 


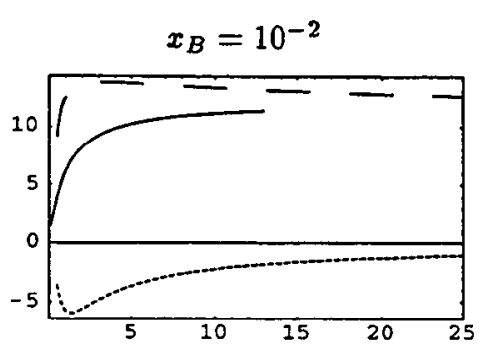

$$
F_{L}\left(Q^{2}\right) A=238(U)
$$
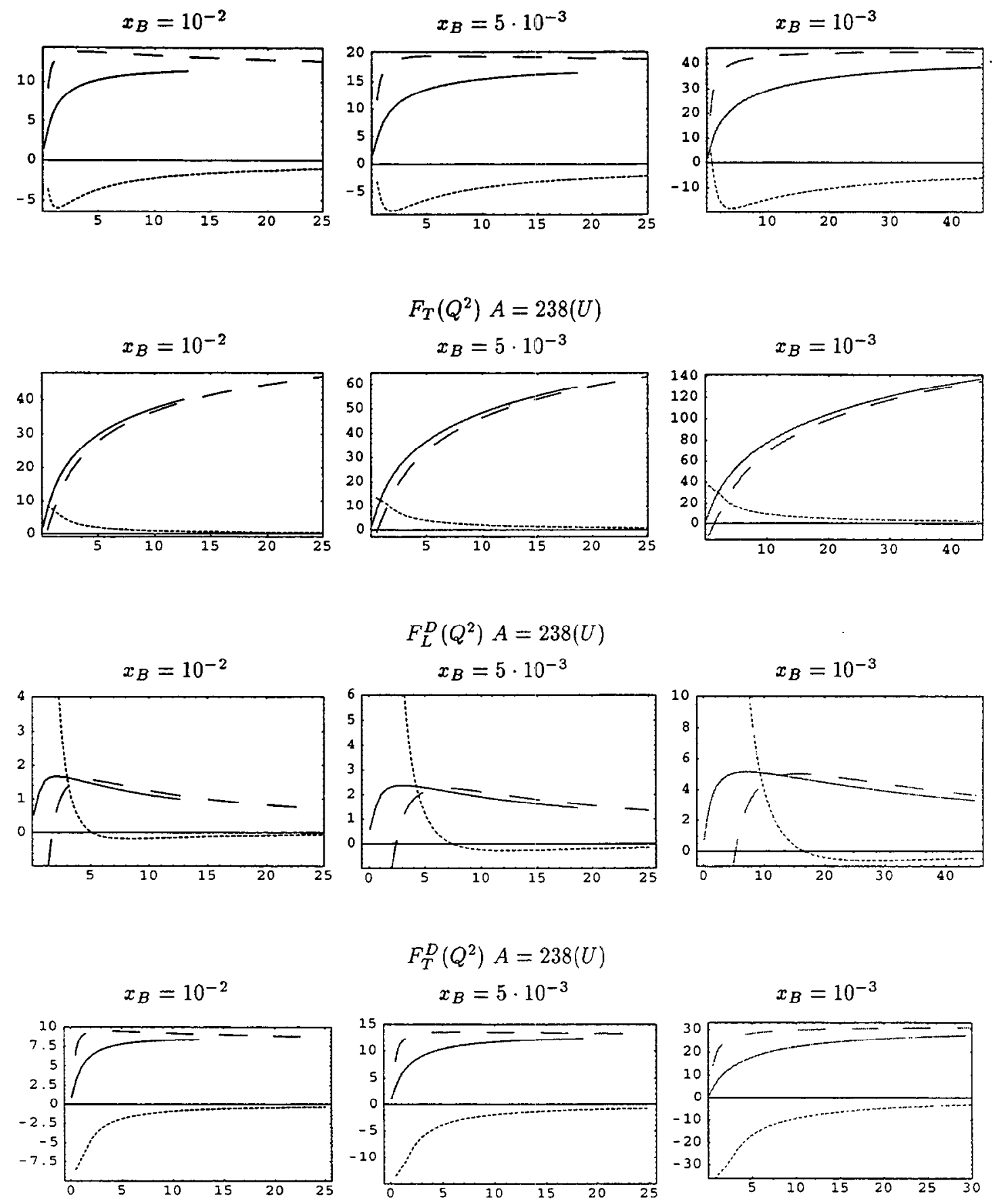

FIG. 11. 
[1] V.N. Gribov, Sov. Phys. JETP 30 (1970) 709.

[2] E. Gotsman, E. Levin and U. Maor, it Nucl. Phys. B464 (1996) 251, B493 (1997) 354, B539 (1999) 535, Eur. Phys. J. C5 (1998) 303, Phys.Lett. B425 (1998) 369;B403 (1997) 120; E. Gotsman, E. Levin, U. Maor and E. Naftali, Nucl.Phys. B539 (1999) 535, Eur.Phys.J. C10 (1999) 689, C14 (2000) 511.

[3] A.L. Ayala Filho, M.B. Gay Ducati and E. Levin, Nucl. Phys. B493 (1997) 305, B511 (1998) 355, Phys. Lett. B388 (1996) 188.

[4] E. Gotsman, E. Ferreira, E. Levin, U. Maor and E. Naftali, "Screening corrections in DIS at low $Q^{2}$ and $x$ ", Talk given at 30th International Conference on High-Energy Physics (ICHEP 2000), Osaka, Japan, 27 Jul - 2 Aug 2000; hep-ph/0007274 .

[5] A.H. Mueller, Nucl. Phys. B415 (1994) 373.

[6] E. Levin and L. Frankfurt, JETP Lett. 2 (1965) 65; H. J. Lipkin and F. Scheck, Phys. Rev. Lett. 16 (1966) 71.

[7] A. H. Mueller, Nucl. Phys. B335 (1990) 115,

[8] L.V. Gribov, E.M. Levin and M.G. Ryskin, Phys. Rep 100 (1983) 1; A.H. Mueller and J. Qiu, Nucl. Phys. B268 (1986) 427; L. McLerran and R. Venugopalan, Phys. Rev. D49 (1994) 2233,3352, 50 (1994) 2225, 53 (1996) 458, 59 (1999) 094002.

[9] V. N. Gribov and L. N. Lipatov, Yad. Fiz 15 (1972) 781; L. N. Lipatov, Sov. Phys. J. Nucl. Phys. 20 (1975) 94; G. Altarelli and G. Parisi, Nucl. Phys. B126 (1977) 29; Yu. L. Dokshitzer, Sov. Phys. JETP 46 (1977) 641.

[10] J. Jalilian-Marian, A. Kovner, L. McLerran, and H. Weigert, Phys. Rev. D 55, (1997) 5414; A.H. Mueller, Phys.Lett. B475 (2000) 220, Nucl.Phys. B572 (2000) 227.

[11] Yu. V. Kovchegov, Phys.Rev. D61 (2000) 074018; E. Levin and K. Tuchin, Nucl.Phys. B573 (2000) 833.

[12] E. Levin and M.G. Ryskin, Phys. Rep. 189 (19267) 1990; J.C.Collins and J. Kwiecinski, Nucl. Phys. B335 (1990) 89; J. Bartels, J. Blumlein and G. Shuler, Z. Phys. C50 (1991) 91; E. Laenen and E. Levin, Ann. Rev. Nucl. Part. Sci. 44 (1994) 199 and references therein; A.L. Ayala, M.B. Gay Ducati and E.M. Levin, Nucl. Phys. B493 (1997) 305, Nucl. Phys. B510 (1998) 355; Ia. Balitsky, Nucl.Phys. B463 (1996) 99; Yu. Kovchegov, Phys. Rev. D54 (191996) 5463, Phys. Rev. D55 (191997) 5445, Phys. Rev. D60 (191000) 034008, Phys. Rev. D61 (2000)074018; A.H. Mueller, Nucl. Phys. B572(2000)227, Nucl. Phys. B558 (1999) 285; Yu. V. Kovchegov, A.H. Mueller, Nucl. Phys. B529 (1998) 451; I. Balitsky Nucl. Phys. B463 (1996) 99; E. Levin and K. Tuchin, Nucl. Phys. B573(2000) 833;

[13] J. Jalilian-Marian, A. Kovner, L. McLerran and H. Weigert, Phys. Rev. DD55 (1997) 5414; J. Jalilian-Marian, A. Kovner and H. Weigert, Phys. Rev. D59 (1999) 014015; J. Jalilian-Marian, A. Kovner and H. Weigert, Phys. Rev. D59 (1999) 014015; J. Jalilian-Marian, A. Kovner, A. Leonidov and H. Weigert, Phys. Rev. D59 (1999) 014014,034007, Erratum-ibid. Phys. Rev. D59 (1999) 099903; A. Kovner, J.Guilherme Milhano and H. Weigert, OUTP-00-10P,NORDITA-2000-14-HE, hep-ph/0004014; H. Weigert, NORDITA-2000-34-HE, hep-ph/0004044: M. Braun LU-TP-00-06,hep-ph/0001268.

[14] Ia. Balitsky, Nucl.Phys. B463 (1996) 99; Yu. Kovchegov, Phys. Rev. D60 (191000) 034008.

[15] A. M. Cooper-Sarkar, R. C. E. Devenish and A. De Roeck, Int. J. Mod. Phys. A13 (1998) 33; H. Abramowicz and A. Caldwell, Rev. Mod. Phys. 71 (1999) 1275.

[16] A. Donnachie, P. V. Landshoff, Phys. Lett. B296 (1992) 227; B437(1998) 408 and references therein.

[17] K. Golec-Biernat and M. Wusthoff, Phys. Rev. D59 (1999) 014017; D60 (1999) 114023; K.Golec-Biernat,Talk at 8th International Workshop on Deep Inelastic Scattering and QCD (DIS 2000), Liverpool, England. 25-30 Apr 2000,hep-ph/0006080.

[18] Yu. V. Kovchegov and L. McLerran, Phys.Rev. D60 (1999) 054025

[19] E. Gotsman, E. Levin, M. Lublinsky, U. Maor and K. Tuchin, TAUP-2605-99, hep-ph/9911270.

[20] E. Gotsman, E. Levin, U. Maor, L. McLerran and K. Tuchin, TAUP-2638-200, BNL-NT-00-19, Jul 2000 ,hep-ph/0007258.

[21] A.P. Bukhvostov, G.V. Frolov, L.N. Lipatov and E.A. Kuraev, Nucl. Phys. B258 (1985) 601.

[22] J. Bartels, Phys. Lett. B298 (1993) 204, Z. Phys. C60 (1993) 471; E.M. Levin, M.G. Ryskin and A.G. Shuvaev, Nucl. Phys. B387 (1992) 589.

[23] U.K. Yang and A. Bodek, Phys.Rev.Lett. 84 (2000) 5456, 82 (1999) 2467 and references thertein.

[24] J.Bartels, K. Golec-Biernat and K. Peters, DESY-00-038, Mar 2000,hep-ph/0003042 . 


\title{
Recent Work on Renormalization Group for the Colored Glass Condensate
}

\author{
Larry McLerran \\ Physics Department, Brookhaven National Laboratory, Upton, NY 11979, USA
}

October 19,2000

\begin{abstract}
In this brief report, I summarize the recent work which I have been doing with E. Iancu and A. Leonidov to get explicit formulae for the renormalization group equations which describe the Colored Glass Condensate.
\end{abstract}

\section{The Problem}

The Colored Glass Condensate is a theory of colored gluons in the presence of a light cone source $J \mu\left(x^{-}, x^{+}\right)=\delta^{\mu+} \rho\left(x^{-}, x_{T}\right)$, where one averages over all possible orientations of $\rho[1]-[12]$. The functional measure for this theory is

$$
Z=\int[d \rho] \int[d A] \exp p^{i S(A, \rho)}
$$

This system in weak coupling describe a bose condensed state of gluons. The averaging over sources is how one describes glasses. Hence the name, a colored glass condensate.

This theory is an effective theory. If we let $p^{-}$be a light cone momentum for any degree of freedom for the theory, then we require that $p^{-}>>p_{0}^{-} \cdot[13]$

The effective action is a generalization of that for a system in an external current

$$
S=\frac{1}{4} F^{2}+\frac{i}{N_{c}} \int d^{3} x \operatorname{Tr} \rho(x) P e^{i \int_{-\infty}^{+\infty} d x^{+} A^{-}}
$$

Here the field $A^{-}$within the path ordered phase is in an adjoint representation of the color group. This provides a gauge invariant generalization for the usual source action for a fixed external current.

Due to the mass shell constraint, particles in this theory would satisfy $p^{+} p^{-}=p_{T}^{2} / 2$, so that the constraint for mass shell and near mass shell modes is equivalent to $p^{+}<p_{T}^{2} / 2 p_{0}^{-}$. 
The theory therefore should describe small $\mathrm{x}$ modes. Iancu, Leonidov and I have shown that the leading terms in the small $x$ effective action are in fact generated by such almost on shell modes.[13]

The classical equations for this theory,

$$
D_{\mu} F^{\mu \nu}=\delta^{\nu+} \rho\left(x^{-}, x_{T}\right)
$$

have a simple solution with $A^{-}-A^{+}=0$ in light cone gauge. To see this solution it is first convenient to write the solution in covariant gauge and then rotate back to light cone gauge. In covariant gauge,

$$
A^{i}=A^{-}=0
$$

and

$$
-\nabla_{T}^{2} A^{+}=\rho
$$

Returning to light cone gauge involves the gauge rotation $A^{\mu} \rightarrow i U \partial^{\mu} U^{\dagger}+U A^{\mu} U^{\dagger}$, where $U$ solves

$$
-i \partial^{+} U^{\dagger}=A^{+} U^{\dagger}
$$

The solution to this equation is only specified if we impose a boundary condition

$$
\lim _{x^{-} \rightarrow-\infty} U^{\dagger}=1
$$

so that

$$
U^{\dagger}=\operatorname{Pcxp}\left\{\int_{-\infty}^{x^{-}} d x^{-1} \frac{1}{-\nabla_{T}^{2}} \rho\right\}
$$

This boundary condition is associated with our gauge fixing prescription It is essentially a retarded prescription for defining the inverse of $\partial^{+}$

The gauge rotated light cone field is of the form

$$
A^{2}=i U \nabla_{T}^{2} U^{\dagger}
$$

This is in the 2-dimensional transverse subspace, a pure gauge, so we have

$$
F^{i j}=0
$$

It has no $x^{+}$dependence so

$$
F^{i-}=0
$$

It has a non-zero $F^{2+}$. These equations inply that $F^{20}=F^{i z}$ so that the field has $|\vec{E}|=|\vec{B}|$ and

$$
\vec{z} \perp \vec{E} \perp \vec{B}
$$

These fields are therefore the generalization of the Lienard-Wiechart potentials to clectrodynamics from QCD. They describe almost on mass shell gluons which are plane polarized to their direction of motion (like massless spin one particles). 


\section{The Renormalization Group}

The results one can derive from the above classical considerations are true if $p^{-}$is not too much greater than $p_{0}^{-}$. How do the classical considerations breakdown?

If one computes

$$
<A A>=<A_{c l} A_{c l}>+<A_{c l} A_{q}>+<A_{q} A_{c l}>+<A_{q} A_{q}>
$$

where $A_{c l}$ is the classical field and $A_{q}$ is the quantum, then one gets a correction both to the quantites $\left\langle A_{q}>\right.$ and $\left\langle A_{q} A_{q}\right\rangle$. The first is associated with vacuum polarization and we will call it a virtual piece, and the second is associated with computing the quantum propogators, ie the connected piece of the propogators, and will be called the real piece. Both terms involve corrections which are of order $\alpha_{s} \ln (1 / x)$ Therefore if

$$
e^{-1 / \alpha_{s}} x_{\text {upper }}<<x<<x_{\text {upper }}
$$

where $x_{\text {upper }}$ is the upper-cutoff in $x$ associated with the $p^{-}$cutoff described in the first section.

This presents a problem if we are to use this theory to describe dynamics at very small $\mathrm{x}$. The way around this is to use the fact that in a small interval of $x$ where the quantum corrections are small, one may integrate out the quantum fluctuations. This can be done consistently in weak coupling. This generates a theory at a smaller cutoff scale. One can iterate this procedure. The iteration is essentially the renormalization group.

One can show that the only effect of integrating out the quantum fluctuations at an intermediate scale is to change

$$
F[\rho] \rightarrow F^{\prime}[\rho]
$$

This is a functional transformation of the theory. The renormalization group gives functional differential equations for $F$.

The coefficients of this functional renormalization group equation involve loop integrals over propogators to all orders in the background field generated by the source. It appears to be a very difficult task to compute these terms. In fact, to define them one must be extremely careful to both completely specify the gauge and to smear out the field in $x^{-}$. After much work, lancu, Leonidov and I have computed these terms explicitly. We found no way to make sense of them unless we had them smeared out, and worked in a gauge where the inverse of $\partial^{+}$was defined by a retarded prescription. (Advanced would also have worked.) We can demonstrate the gauge invariance of the effective action which results from our prescription.[13]

Since fixing a gauge is so essential, we must ask whether we can express the objects of interest in a gauge invariant manner. In fact one can always write down light cone 
gauge operators in a gauge invariant way by inserting line ordered phases between the coordinates of interest in the operators. The problem with this procedure is that it then appears that the gauge invariant operators depend upon the path used to define them.

The operators of interest here involve measurements on scales much larger than the extent of the sources. Outside the source, the field is a pure two dimensional gauge. The expectation values of operators therefore do not depend upon the way one joins points together in the two dimensional subspace. This is sufficient to prove that for the operators of interest, they are both gauge invariant and path independent as a consequence of the special form of the classical fields.

We have used our result to compare with the results of that of Balitsky and Kovchegov for the large $N_{c}$ limit for the correlation function of two line ordered phases. It can be argued that these line ordered phases generate $F_{2}$ in deep inelastic scattering. They argue that the equation for $F_{2}$ is a closed non-linear integral equation which can be solved numerically. Our results agree with the form of the equation by Balitsky and Kovchegov,[14]-[15] and disagree with the analysis of Kovner et. al.[16] We believe the problem is in the way that Kovner et. al. fix their gauge.

If the large $N_{c}$ limit is simple for the two point function, then there may be some hope that one can solve the functional renormalization group equations for $F$ at least in the large $N$ limit. That is, the small $x$ limit is exactly solvable in large $N_{c}$.

\section{Acknowledgements}

I gratefully acknowledge the efforts of my collaborators on this protracted project. I also acknowledge the effort of all the Minnesota Mob which has been working on the small $\mathrm{x}$ problem. (You know who you are, and so does everyone else.) My work was supported by the US Department of Energy (Contract \# DE-AC02-98CH10886).

\section{References}

(1] L. McLerran and R. Venugopalan, Phys. Rev. D49 (1994), 2233; 49 (1994) 3352; 50 (1994), 2225

[2] Yu.V. Kovchegov, Phys. Rev. D54 (1996), 5463; Phys. Rev. D55 (1997), 5445

[3] A. H. Mueller, Nucl. Phys. B437, (1995) 107.

[4] A. H. Mueller, Nucl. Phys. B415, (1994) 373.

[5] L. McLerran and R. Venugopalan, Phys. Rev. D59, (1999) 094002. 
[6] A. Kovner, L. McLerran and H. Weigert, Phys. Rev. D52, (1995) 6231; D52, (1995) 3809 .

[7] W. Buchmuller, T. Gehrmann and A. Hebecker, Nucl. Phys. B537, (1999) 477.

[8] Y. Kovchegov and L. McLerran, Phys. Rev. D60, (1999), 054025.

[9] J. Jalilian-Marian, A. Kovner, L. McLerran and H. Weigert, Phys. Rev. D55 (1997), 5414

[10] J. Jalilian-Marian, A. Kovner, A. Leonidov and H. Weigert, Nucl. Phys. B504 (1997), 415

[11] J. Jalilian-Marian, A. Kovner, A. Leonidov and H. Weigert, Phys. Rev. D59 (1999), 014014

[12] J. Jalilian-Marian, A. Kovner, A. Leonidov and H. Weigert, Phys. Rev. D59 (1999), 034007; Erratum-ibid. D59 (1999), 099903

[13] E. Iancu, A. Leonidov and L. McLerran, work in progress.

[14] I. Balitski, Nucl. Phys. B463 (1996), 99

[15] Y. Kovchegov, Phys. Rev. D61, (2000) 074018.

[16] A. Kovner, J. Guilherme Milhano and H. Weigert Phys. Rev. D62, (2000), 114005. 


\title{
Parton Energy Loss and Modified Fragmentation Functions in Deeply Inelastic $e A$ Scattering
}

\author{
Xin-Nian Wang \\ Nuclear Science Division, Mailstop 70-319, \\ Lawrence Berkeley National Laboratory, Berkeley, CA 94720 USA
}

Octuber 29, 2000

\begin{abstract}
Gluon radiation induced by multiple scattering leads to modified quark fragmentation functions in deeply inelastic $e A$ collisions. Such modified fragmentation functions and their QCD evolution equations are derived for the first time in the framework of multiple parton scattering. The induced radiation gives rise to additional terms in the evolution equations and thus softens the modified quark fragmentation functions that can be directly translated into the energy loss of the leading quark. The results in the next-leading-twist depend on both diagonal and off-diagonal twist-four parton distributions and the combination of which clearly manifests the LPM interference pattern. The predicted modification to the fragmentation functions depends quadratically on the nuclear size $\left(A^{2 / 3}\right)$. Generalization of our results to the case of hot QCD medium predicts sensitive dependence of the modification to the parton correlation length which could have a dramatic change during a QCD phase transition.
\end{abstract}

The propagation of an energetic parton and its induced energy loss has been proposed as a probe of the properties of dense matter formed in highenergy nuclear collisions $[1,2]$. Recent theoretical studies $[3,4,5,6]$ show that a fast parton will lose a significant amount of energy via induced radiation 
when it propagates through a hot partonic matter. One cannot directly measure the energy loss of partons because they are not final experimentally observed particles. However, parton energy loss does lead to modification of the final particle spectra. Therefore, one can only study the parton energy loss indirectly by measuring the modification of the parton fragmentation functions in semi-inclusive processes like $e A$ or $\gamma$-jet events in $A A$ collisions [8] or the inclusive spectra at large transverse momentum $[2,9]$.

In this Letter, we report our first study and derivation of the QCD evolution equations for the medium-modified fragmentation functions in the simplest case of deeply inelastic $e A$ scattering (DIS). The induced gluon radiation due to multiple parton scattering gives rise to additional terms in the modified QCD evolution equations that soften the modified fragmentation functions. Utilizing the generalized factorization of higher-twist (HT) parton distributions [10], we show that these additional HT terms depend on both the diagonal and off-diagonal twist-four parton distributions, the combination of which clearly manifests the LPM interference pattern. Using estimates of these twist-four parton matrix elements from other processes such as the $p_{T}$ broadening of Drell-Yan dilepton in $p A$ collisions, we predict the modification of the effective quark fragmentation functions and their dependence on the parton energy and nuclear size. We also estimate the quark energy loss defined as the total energy carried by gluons from induced radiation.

We consider the following semi-inclusive process in the deeply inelastic $e A$ scattering, $e\left(L_{1}\right)+A(p) \longrightarrow e\left(L_{2}\right)+h\left(\ell_{h}\right)+X$, where $L_{1}$ and $L_{2}$ are the four momenta of the incoming and the outgoing leptons, $\ell_{h}$ is the observed hadron momentum, $p$ and $q=L_{2}-L_{1}$ denoted as $p=\left[p^{+}, 0, \mathbf{0}_{\perp}\right], q=$ $\left[-Q^{2} / 2 q^{-}, q^{-}, 0_{\perp}\right]$, are the momentum per nucleon in the nucleus with the atomic number $A$ and the momentum transfer, respectively. The differential cross section for the semi-inclusive process can be expressed as

$$
E_{L_{2}} E_{\ell_{h}} \frac{d \sigma_{\mathrm{DIS}}^{h}}{d^{3} L_{2} d^{3} \ell_{h}}=\frac{\alpha_{\mathrm{EM}}^{2}}{2 \pi s} \frac{1}{Q^{4}} L_{\mu \nu} E_{\ell_{\ell_{h}}} \frac{d W^{\mu \nu}}{d^{3} \ell_{h}}
$$

where $s=\left(p+L_{1}\right)^{2}$ and $\alpha_{\mathrm{EM}}$ is the electromagnetic (EM) coupling constant. The leptonic tensor is given by $L_{\mu \nu}=1 / 2 \operatorname{Tr}\left(\gamma \cdot L_{1} \gamma_{\mu} \gamma \cdot L_{2} \gamma_{\nu}\right)$ while the semi-inclusive hadronic tensor is defined as,

$$
E_{\ell_{h}} \frac{d W_{\mu \nu}}{d^{3} \ell_{h}}=\frac{1}{2} \sum_{X}\left\langle A\left|J_{\mu}(0)\right| X^{-}, h\right\rangle\left\langle X, h\left|J_{\nu}(0)\right| A\right\rangle
$$




$$
\times 2 \pi \delta^{4}\left(q+p-p_{X}-\ell_{h}\right)
$$

where $\sum_{X}$ runs over all possible final states and $J_{\mu}=\sum_{q} e_{q} \bar{\psi}_{q} \gamma_{\mu} \psi_{q}$ is the hadronic EM current.

In the parton model with collinear factorization approximation and to the leading-twist (LT) the semi-inclusive cross section factorizes into a product of parton distributions, parton fragmentation functions and the partonic cross section. Therefore, to the leading order in $\alpha_{\mathrm{s}}$,

$$
\begin{aligned}
& \frac{d W_{\mu \nu}^{S}}{d z_{h}}=\sum_{q} e_{q}^{2} \int d x f_{q}^{A}\left(x, \mu_{I}^{2}\right) H_{\mu \nu}^{(0)}(x, p, q) D_{q \rightarrow h}\left(z_{h}, \mu^{2}\right) \\
& H_{\mu \nu}^{(0)}(x, p, q)=\frac{1}{2} \operatorname{Tr}\left(\gamma \cdot p \gamma_{\mu} \gamma \cdot(q+x p) \gamma_{\nu}\right) \frac{2 \pi}{2 p \cdot q} \delta\left(x-x_{B}\right),
\end{aligned}
$$

where the momentum fraction carried by the hadron is defined as $z_{h}=\ell_{h}^{-} / q^{-}$ and $x_{B}=Q^{2} / 2 p^{+} q^{-}$is the Bjorken variable. $\mu_{I}^{2}$ and $\mu^{2}$ are the factorization scales for the initial quark distributions $f_{q}^{A}\left(x, \mu_{I}^{2}\right)$ in a nucleus and the fragmentation functions $D_{q \rightarrow h}\left(z_{h}, \mu^{2}\right)$, respectively. Including all leading log radiative corrections, the renormalized quark fragmentation function $D_{q \rightarrow h}\left(z_{h}, \mu^{2}\right)$ satisfies the QCD evolution equation [11].

In this paper we will consider contributions of quark rescattering with partons from another nucleon inside the nucleus. Such contributions are proportional to the nuclear size $A^{1 / 3}$ [10]. For large enough $A$, we can neglect other $A$-independent HT effects. For large $Q^{2}$ in DIS, it may suffice to only consider one rescattering. The contributions of one rescattering can be treated as HT corrections to the LT results. We work in a framework [13] in which the twist-four contributions can be expressed as the convolution of the partonic hard parts and four-parton matrix elements [10]. At the lowest order, rescattering without gluon radiation as shown in Fig. 1(a) broadens the transverse momentum of the leading jet [12] but contribute little to parton energy loss. One can also neglect rescattering with another quark in Fig. 1(b).

The dominant HT contributions to the QCD evolution of the fragmentation functions come from radiative processes involving rescattering with a gluon from another nucleon as illustrated by the central-cut diagram in Fig. 1(c). Kinematics only allows two poles, one at each side of the centralcut, out of the four propagators in the diagram. This leads to four possible combinations each give different momentum fractions to the initial partons. 

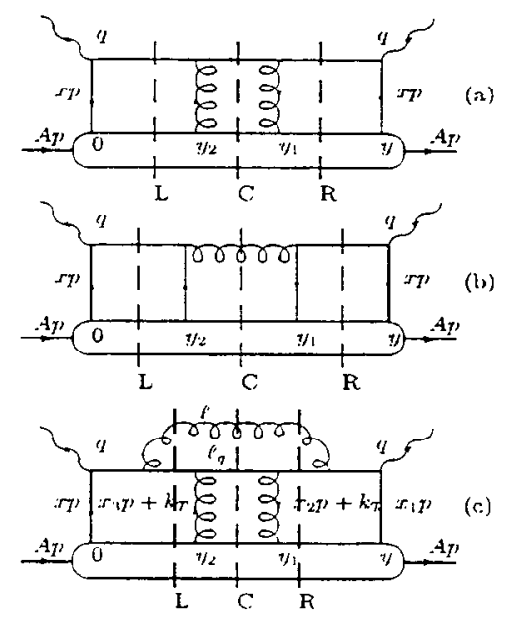

Figure 1: Diagrams for rescattering with gluons (a) and quarks (b) without and with gluon radiation (c) in deeply inelastic eA scattering. Possible cuts are shown by the dashed lines

In one case, the initial gluon has $x_{2}=x_{L}+x_{D}$ which is finite when $k_{T} \rightarrow 0$, where

$$
x_{L}=\frac{\ell_{T}^{2}}{2 p^{+} q^{-} z(1-z)} ; x_{D}=\frac{k_{T}^{2}-2 \vec{k}_{T} \cdot \vec{\ell}_{T}}{2 p^{+} q^{-} z},
$$

$\ell_{T}$ is the transverse momentum of the radiated gluon, $k_{T}$ is the initial gluon's intrinsic transverse momentum, and $z=\ell_{q}^{-} / q^{-}$is the momentum fraction carried by the final quark. This corresponds to gluon radiation induced by the rescattering and is referred to as a double-hard process. In another combination, $x_{2}=x_{D}$ which vanishes when $k_{T} \rightarrow 0$. In this case the rescattering is soft and the gluon radiation is induced by the initial hard photon-quark scattering. Such a process is called hard-soft. The four contributions from Fig. 1(c) correspond to these two distinct processes and their interferences. Their sum has the form,

$$
\begin{aligned}
H_{\mu \nu}^{D(1)} & \propto\left(1-e^{-i x_{L} p^{+} y_{2}^{-}}\right)\left(1-e^{-i x_{L} p^{+}\left(y^{-}-y_{1}^{-}\right)}\right) \\
& \times e^{2 x_{D} p^{+}\left(y_{1}^{-}-y_{2}^{-}\right)} .
\end{aligned}
$$

This clearly manifests the LPM interference pattern caused by the destructive interferences between hard-soft and double-hard processes. The interference 
pattern is dictated by the gluon's formation time, $\tau_{f} \equiv 1 / x_{L} p^{+}$, relative to the nuclear size. The two processes completely cancel each other in the collinear limit when $\ell_{T} \rightarrow 0$. Diagrams involving three-gluon vertices have exactly the same structure as Fig. 1(c), except that they have different momentum dependence and color factor in the hard part.

We have considered all together 23 possible cut diagrams, 14 of them are interferences between no and double rescattering (shown as the left and right-cut diagrams in Fig. 1(c)) which cancel some of the contributions from central-cut diagrams. Including virtual corrections, we obtain [14] the leading HT contribution from rescattering processes,

$$
\begin{aligned}
\frac{d W_{\mu \nu}^{D}}{d z_{h}} & =\sum_{q} e_{q}^{2} \int d x H_{\mu \nu}^{(0)}(x, p, q) \frac{2 \pi \alpha_{\mathrm{s}}}{N_{c}} \int \frac{d \ell_{T}^{2}}{\ell_{T}^{4}} \int_{z_{h}}^{1} \frac{d z}{z} \\
& \times D_{q \rightarrow h}\left(z_{h} / z\right) \frac{\alpha_{\mathrm{s}}}{2 \pi} C_{A}\left[\frac{1+z^{2}}{(1-z)_{+}} T_{q g}^{A}\left(x, x_{L}\right)\right. \\
& \left.+\delta(z-1) \Delta T_{q g}^{A}\left(x, \ell_{T}^{2}\right)\right]
\end{aligned}
$$

where

$$
\begin{aligned}
T_{q g}^{A}\left(x, x_{L}\right) & =\int \frac{d y^{-}}{2 \pi} d y_{1}^{-} d y_{2}^{-} e^{i\left(x+x_{L}\right) p^{+} y^{-}+i x_{T} p^{+}\left(y_{1}^{-}-y_{2}^{-}\right)} \\
& \frac{1}{2} \quad\left\langle A\left|\bar{\psi}_{q}(0) \gamma^{+} F_{\sigma}^{+}\left(y_{2}^{-}\right) F^{+\sigma}\left(y_{1}^{-}\right) \psi_{q}\left(y^{-}\right)\right| A\right\rangle \\
& \times\left(1-e^{-i x_{L} p^{+} y_{2}^{-}}\right)\left(1-e^{-i x_{L} p^{+}\left(y^{-}-y_{1}^{-}\right)}\right) \\
& \times \theta\left(-y_{2}^{-}\right) \theta\left(y_{2}^{-}-y_{1}^{-}\right)
\end{aligned}
$$

is quark-gluon correlation function which essentially contains four independent four-parton matrix elements in a nucleus and $x_{T}=\left\langle k_{T}^{2}\right\rangle / 2 p^{+} q^{-}=$ $x_{B}\left\langle k_{T}^{2}\right\rangle / Q^{2}$. With the definition of the + functions [15], the term proportional to the $\delta$-function accounts for virtual corrections and

$$
\begin{aligned}
\Delta T_{q g}^{A}\left(x, \ell_{T}^{2}\right) & \equiv \int_{0}^{l} d z \frac{1}{1-z}\left[\left.2 T_{q g}^{A}\left(x, x_{L}\right)\right|_{z=1}\right. \\
& \left.-\left(1+z^{2}\right) T_{q g}^{A}\left(x, x_{L}\right)\right] .
\end{aligned}
$$

One can similarly get the contribution from the gluon fragmentation. We will neglect the radiative corrections to processes such as Fig. 1(b) that involve 
rescattering with a quark in the leading log approximation, because they can be shown to be proportional to $1 / \ell_{T}^{2}[14]$ as compared to $1 / \ell_{T}^{4}$ in Eq. (6).

Summing up all the leading contributions from LT and HT processes, we can effectively define the modified quark fragmentation function as

$$
\frac{d W_{\mu \nu}}{d z_{h}}=\sum_{q} e_{q}^{2} \int d x f_{q}^{A}\left(x, \mu_{I}^{2}\right) H_{\mu \nu}^{(0)}(x, p, q) \widetilde{D}_{q \rightarrow h}\left(z_{h}, \mu^{2}\right) .
$$

where for completeness $f_{q}^{A}\left(x, \mu_{I}^{2}\right)$ should also include the HT contributions as studied by Mueller and Qiu [16]. The modified quark fragmentation function satisfies the following evolution equation

$$
\begin{aligned}
\frac{\partial \widetilde{D}_{q}\left(z_{h}, \mu^{2}\right)}{\partial \ln \mu^{2}} & =\frac{\alpha_{\mathrm{s}}}{2 \pi} \int_{z_{z_{\iota}}}^{1} \frac{d z}{z}\left[\widetilde{\gamma}_{q \rightarrow q g}\left(z, x, x_{L}, \mu^{2}\right) \widetilde{D}_{q}\left(z_{h} / z, \mu^{2}\right)\right. \\
& \left.+\widetilde{\gamma}_{q \rightarrow g q}\left(z, x, x_{L}, \mu^{2}\right) D_{g}\left(z_{h} / z, \mu^{2}\right)\right]
\end{aligned}
$$

with the modified splitting functions defined as

$$
\begin{aligned}
\tilde{\gamma}_{q \rightarrow q g}\left(z, x, x_{L}, \ell_{T}^{2}\right) & =\gamma_{q \rightarrow q g}(z)+\Delta \gamma_{q \rightarrow q g}\left(z, x, x_{L}, \ell_{T}^{2}\right) \\
\Delta \gamma_{q \rightarrow q g}\left(z, x, x_{L}, \ell_{T}^{2}\right) & =\frac{2 \pi \alpha_{S} C_{A}}{\ell_{T}^{2} N_{c} f_{q}^{A}\left(x, \mu_{I}^{2}\right)}\left[\frac{1+z^{2}}{(1-z)_{+}} T_{q g}^{A}\left(x, x_{L}\right)\right. \\
& \left.+\delta(1-z) \Delta T_{q g}^{A}\left(x, \mu^{2}\right)\right] \\
\tilde{\gamma}_{q \rightarrow g q}\left(z, x, x_{L}, \ell_{T}^{2}\right) & =\tilde{\gamma}_{q \rightarrow q g}\left(1-z, x, x_{L}, \ell_{T}^{2}\right),
\end{aligned}
$$

where $\gamma_{q \rightarrow q g}(z)$ is the normal splitting functions [11]. We assume in the leading order that the gluon fragmentation function follows the normal QCD evolution equations.

Solving the above equation is equivalent to summing all leading log twistfour contributions and will be discussed in a separate publication [14]. As an approximation, one can write the solution as,

$$
\begin{aligned}
\widetilde{D}_{q \rightarrow h}\left(z_{h}, \mu^{2}\right) & \equiv D_{q \rightarrow h}\left(z_{h}, \mu^{2}\right)+\Delta D_{q \rightarrow h}\left(z_{h}, \mu^{2}\right) \\
\Delta D_{q \rightarrow h}\left(z_{h}, \mu^{2}\right) & =\frac{\alpha_{\mathrm{s}}}{2 \pi} \int_{0}^{\mu^{2}} \frac{d \ell_{T}^{2}}{\ell_{T}^{2}} \int_{z_{h}}^{1} \frac{d z}{z} \\
& \times\left[\Delta \gamma_{q \rightarrow q g}\left(z, x, x_{L}, \ell_{T}^{2}\right) D_{q \rightarrow h}\left(z_{h} / z, \mu^{2}\right)\right. \\
& \left.+\Delta \gamma_{q \rightarrow g q}\left(z, x, x_{L}, \ell_{T}^{2}\right) D_{g \rightarrow h}\left(z_{h} / z, \mu^{2}\right)\right]
\end{aligned}
$$


where $D_{a \rightarrow h}\left(z_{h}, \mu^{2}\right)$ are the normal fragmentation functions. Notice that there is no collinear divergence in the above integration because of the LPM effect in $T_{q g}^{A}$. Because $\Delta \gamma$ is proportional to $1 / \ell_{T}^{2}, \Delta D_{q \rightarrow h}$ is suppressed by $1 / \mu^{2}$ relative to the LT fragmentation function $D_{q \rightarrow h}$.

To estimate the twist-four parton matrix elements, we generalize the approach by [10] to include the off-diagonal matrix elements. Assuming a Gaussian nuclear distribution in the rest frame, $\rho(r) \sim \exp \left(-r^{2} / 2 R_{A}^{2}\right)$, $R_{A}=1.12 A^{1 / 3} \mathrm{fm}$, we express $T_{q g}^{A}$ in terms of single parton distributions,

$$
\begin{aligned}
T_{q g}^{A}\left(x, x_{L}\right) & =\frac{C}{x_{A}}\left[f_{q}^{A}(x)\left(x_{T}+x_{L}\right) G\left(x_{T}+x_{L}\right)\right. \\
& \left.+f_{q}^{A}\left(x+x_{L}\right) x_{T} G\left(x_{T}\right)\right]\left(1-e^{-x_{L}^{2} / x_{A}^{2}}\right),
\end{aligned}
$$

where $G(x)$ is the gluon distribution per nucleon in a nucleus, $x_{A}=1 / M R_{A}$, and $M$ is the nucleon's mass. The off-diagonal terms involves transferring momentum $x_{L}$ between different nucleons inside a nucleus and thus should be suppressed for large nuclear size or large momentum fraction $x_{L}$. Notice that $\tau_{f}=1 / x_{L} p^{+}$is the gluon's formation time. Thus, $x_{L} / x_{A}=L_{A} / \tau_{f}$ with $L_{A}=R_{A} M / p^{+}$being the nuclear size in our chosen frame.

Using the above approximation in Eq. (14) and replacing the momentum fraction $x_{L}$ in the parton distributions by its average value $\left\langle x_{L}\right\rangle \sim x_{A}$, we have

$$
\begin{aligned}
\Delta D_{q \rightarrow h}\left(z_{h}, Q^{2}\right) & \approx \frac{C_{A} \alpha_{s}^{2}}{N_{c}} \frac{\tilde{C}}{Q^{2} x_{A}^{2}} \Delta d_{q \rightarrow h}\left(z_{h}, x_{B}, x_{A}, Q^{2}\right), \\
\tilde{C} & =C\left[x_{T} G\left(x_{T}\right)+\left(x_{T}+x_{A}\right) G\left(x_{T}+x_{A}\right)\right],
\end{aligned}
$$

where we choose the factorization scale $\mu^{2}=Q^{2}$. Shown in Fig. 2 are the numerical results of $\Delta d\left(z_{h}, x_{B}, x_{A}, Q^{2}\right)$ for three different values of $x_{B}$. The parameterization in Ref. [17] of the normal fragmentation functions is used in our calculation. As we see from the numerical results, the modification to the shape of fragmentation function increases for larger values of $x_{B}$ corresponding to smaller quark energy $q^{-}$at fixed $Q^{2}$. The modification also increases for smaller values of $x_{A}$ corresponding to larger nuclear size. As shown in Eq. (16), the magnitude of the modification depends quadratically on the nuclear size. This is because the LPM effect modifies the transverse momentum spectra of the radiated gluon such that the phase space for the 


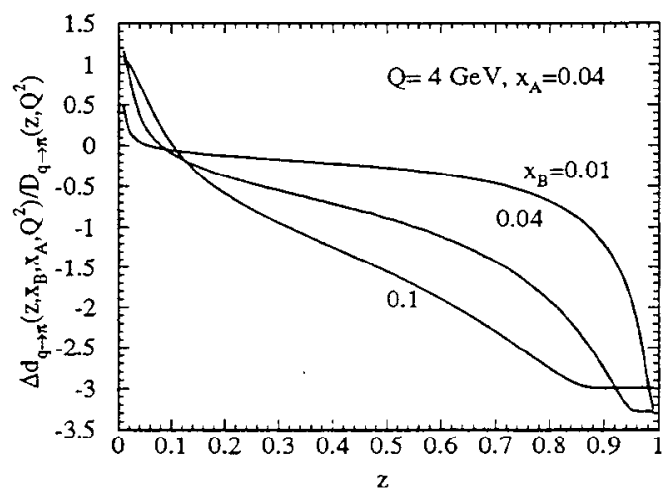

Figure 2: The predicted modification to the quark fragmentation functions for three different values of initial quark energy $q^{-}=Q^{2} / 2 p^{+} x_{B} \cdot x_{A}=0.04$ corresponds to $A \approx 200$

momentum integral is limited. That limited phase space is proportional to the nuclear size. Together with the linear dependence of the parton correlation function, this leads to non-linear dependence of the total energy loss on the nuclear size.

We can define the quark energy loss as the momentum carried by the emitted gluons. From Eq. (14), we have,

$$
\begin{aligned}
\left\langle\Delta z_{g}\right\rangle & =\int_{0}^{Q^{2}} \frac{d \ell_{T}^{2}}{\ell_{T}^{2}} \int_{0}^{1} d z \frac{\alpha_{\mathrm{s}}}{2 \pi} z \Delta \gamma q \rightarrow g q \\
& \approx \frac{C_{A} \alpha_{\mathrm{s}}^{2}}{N_{c}} \frac{\widetilde{C}}{Q^{2}} \frac{x_{B}}{x_{A}^{2}} 6 \ln \left(\frac{1}{2 x_{B}}\right), \quad\left(x_{A} \ll \ell_{T}^{2}\right)
\end{aligned}
$$

According to Ref. [18, 19], $\widetilde{C}$ can be related to the transverse momentum broadening of Drell-Yan dilepton in $p A$ collisions,

$$
\Delta\left\langle k_{T}^{2}\right\rangle_{\mathrm{DY}} \approx \frac{2 \pi \alpha_{\mathrm{s}}}{N_{\mathrm{c}}} \frac{\widetilde{C}}{2 x_{A}} \approx 0.022 A^{1 / 3} \mathrm{GeV}^{2}
$$

In the rest frame of the nucleus, the total energy loss is

$$
\Delta E=q_{0}\left\langle\Delta z_{g}\right\rangle \approx 0.35 \alpha_{s} A^{2 / 3} \ln \frac{1}{2 x_{B}} \mathrm{GeV}
$$


which depends quadratically on the nuclear size. For $x_{B}=0.1, \alpha_{\mathrm{S}}=0.3$ and $A=200, \Delta E \approx 5.8 \mathrm{GeV}$ which is consistent with the estimate in Ref [4].

In summary, we have derived for the first time the evolution equations for the nuclear modified quark fragmentation functions due to multiple scattering and induced radiation. The final result clearly manifests the LPM interference effect and the modification depends quadratically on the nuclear size. We can generalize our results to the case of hot QCD matter. In our approximation, Eq. (15), the quark distribution which represents the initial quark production probability does not enter into the modified evolution equations. What remains is the gluon matrix element. In the case of a hot QCD medium, the coordinates of the two gluon fields are not restricted to the nucleon size due to deconfinement in a nucleus. Rather, the final results will be sensitive to the correlation length of the hot medium [20]. If there is a dramatic change of the correlation length during the QCD phase transition, one should also see a big change in the modification of the parton fragmentation functions.

\section{Acknowledgements}

This work was supported by DOE under Contract No. DE-AC03-76SF00098 and DE-FG-02-96ER40989 and in part by NSFC under project 19928511.

\section{References}

[1] M. Gyulassy and M. Plümer, Phys. Lett. B243, 432 (1990); M. Gyulassy,M. Plümer, M. H. Thoma and X.-N. Wang, Nucl. Phys. A 538 (1992)

[2] X.-N. Wang and M. Gyulassy, Phys. Rev. Lett. 68, 1480 (1992).

[3] M. Gyulassy and X.-N. Wang, Nucl. Phys. B420, 583 (1994); X.-N. Wang, M. Gyulassy and M. Plümer, Phys. Rev. D 51, 3436 (1995).

[4] R. Baier, Yu. L. Dokshitzer, S. Peigné and D. Schiff, Phys. Lett. B345, 277 (1995); R. Baier, Yu. L. Dokshitzer, A. Mueller, S. Peigné and D. Schiff, Nucl. Phys. B484, 265 (1997). 
[5] B. G. Zakharov, JETP Letters, 63, 952 (1996); 65, 516 (1997).

[6] M. Gyulassy, P. Levai, I. Vitev, hep-ph/9907461.

[7] L. D. Landau and I. Ya. Pomeranchuk, Dokl. Akad. Nauk SSSR 92, 535, 735 (1953); A. B. Midgal, Phys. Rev. 103, 1811 (1956).

[8] X.-N. Wang, Z. Huang and I. Sarcevic, Phys. Rev. Lett. 77, 231 (1996);

X.-N. Wang and Z. Huang, Phys. Rev. C 55, 3047 (1997).

[9] X.-N. Wang, Phys. Rev. C 58, 2321 (1998); Phys. Rev. C 61, 064910 (2000).

[10] M. Luo, J.-W. Qiu and G. Sterman, Phys. Lett. B279, 377 (1992); Phys. Rev. D49, 4493 (1994); Phys. Rev. D50, 1951 (1994).

[11] R. Field, Application of Perturbative QCD (Addison-Wesley, USA, 1989).

[12] X.-F. Guo, Phys. Rev. D 58, 114033(1998); X.-F. Guo and J. Qiu, Phys. Rev. D 61, 096003 (2000).

[13] J.-W. Qiu and G. Sterman, Nucl. Phys. B353 (1991) 105, 137.

[14] X.-N. Wang and X. Guo, in preparation.

[15] G. Altarelli and G. Parisi, Nucl. Phys. B126, 298 (1977).

[16] A. Mueller and J. Qiu, Nucl.Phys. B268, 427 (1986).

[17] J. Binnewies, B. A. Kniehl and G. Kramer, Z. Phys. C65, 471 (1995).

[18] X.-F. Guo, Phys. Rev. D 58, 036001(1998).

[19] X. Guo, J. Qiu and X. Zhang, hep-ph/9911476.

[20] X.-N. Wang, Phys. Lett. B485,157 (2000). 


\title{
$A^{\alpha}$ - Physics at eRHIC
}

\author{
Boris Kopeliovich
}

Max-Planck Institut für Kernphysik, Postfach 103980, 69029 Heidelberg

\begin{abstract}
The planned electron-nucleus colliding mode at RHIC would provide an access to many new fine probes for the QCD dynamics and quark-gluon structure of nuclei. The latter needs a good understanding in particular for a proper interpretation of the forthcoming data on heavy ion collisions at RHIC. In this note a few examples of nuclear effects are presented which can be studied at the eRHIC collider:

- nuclear shadowing of quarks and gluons at small $x$;

- highly virtual photoproduction of vector mesons, ground states and radial excitations, as a way to detect the color transparency effects;

- exclusive photoproduction of $J / \Psi$ as a probe for gluon shadowing at small $x$;

- nuclear enhancement of inclusive $J / \Psi$ photoproduction as an evidence for a novel production mechanism;

- inclusive electroproduction of hadrons off nuclei as a way to study the quark fragmentation function in nuclear environment and broadening of the transverse momentum of a quark traveling through the nucleus.
\end{abstract}

\section{Nuclear shadowing in DIS}

\subsection{Coherence length for longitudinal and transverse photons fluc- tuating to $\bar{q} q$ pairs}

Shadowing is the main phenomenon expected for DIS off nuclei in the eRHIC energy range which is two orders of magnitude higher than has been previously achieved in fixed target experiments. Onset of shadowing is controlled by the coherence length $l_{c}$ which is expected to be much longer for longitudinally than for transversely polarized photons, and much shorter for gluon than for quark shadowing [3]. 
The coherence length which can be also interpreted as the lifetime of the corresponding hadronic fluctuation of the virtual photon, is related to $x_{B j}$ as,

$$
l_{c}=\frac{2 \nu}{Q^{2}+M^{2}}=\frac{P}{x_{B j} m_{N}}=P l_{c}^{\max }
$$

where $M$ is the effective mass of the fluctuation, factor $P=\left(1+M^{2} / Q^{2}\right)^{-1}$, and $l_{c}^{\text {max }}=$ $1 / m_{N} x_{B_{\jmath}}$. The widely used approximation is to assume that $M^{2} \approx Q^{2}$ since $Q^{2}$ is the only large dimensional scale available. In this case $P=1 / 2$.

The effective mass of a noninteracting $q$ and $\bar{q}$ is well defined, $M^{2}=\left(m_{q}^{2}+k_{T}^{2}\right) / \alpha(1-\alpha)$, where $m_{q}$ and $k_{T}$ and $\alpha$ are the mass, transverse momentum and fraction of the light-cone momentum of the photon carried by the quark. Therefore, $P$ has a simple form,

$$
P\left(k_{T}, \alpha\right)=\frac{Q^{2} \alpha(1-\alpha)}{k_{T}^{2}+\epsilon^{2}}
$$

where

$$
\epsilon^{2}=\alpha(1-\alpha) Q^{2}+m_{q}^{2} .
$$

We averaged the factor $P\left(k_{T}, \alpha\right)$ properly weighted with the $\bar{q} q$ light-cone wave function squared and realistic dipole cross section $[2,1]$,

$$
\left\langle P^{T, L}\right\rangle=\frac{\int_{0}^{1} d \alpha \int d^{2} r_{1} d^{2} r_{2}\left[\Psi_{q \bar{q}}^{T, L}\left(\vec{r}_{2}, \alpha\right)\right]^{*} \sigma_{q \bar{q}}^{N}\left(r_{2}, s\right) \tilde{P}\left(\vec{r}_{2}-\vec{r}_{1}, \alpha\right) \Psi_{q \bar{q}}^{T, L}\left(\vec{r}_{1}, \alpha\right) \sigma_{q \bar{q}}^{N}\left(r_{1}, s\right)}{\int_{0}^{1} d \alpha \int d^{2} r\left|\Psi_{q \bar{q}}^{T, L}(\vec{r}, \alpha) \sigma_{q \bar{q}}^{N}(r, s)\right|^{2}}
$$

with

$$
\tilde{P}\left(\vec{r}_{2}-\vec{r}_{1}, \alpha\right)=\int \frac{d^{2} k_{T}}{(2 \pi)^{2}} \exp \left(-i \vec{k}_{T} \cdot\left(\vec{r}_{2}-\vec{r}_{1}\right)\right) P\left(\alpha, k_{T}\right)-\frac{Q^{2} \alpha(1-\alpha)}{2 \pi} K_{0}\left(\varepsilon\left|\vec{r}_{2}-\vec{r}_{1}\right|\right) .
$$

The results are depicted in Fig. 1 as function of $x_{B j}$ at $Q^{2}=4$ and $40 G e V^{2}$ by solid and dashed lines respectively. The values of $P^{T, L}$ are quite different from the usual estimate $P^{T, L}=1 / 2$. In particular, $P^{L}$ turns out to be substantially longer than $P^{T}$. This indicates that a longitudinally polarized photon develops lighter fluctuations than a transverse one.

The light-cone Green function approach developed in [1] allows to extend these calculations down to $Q^{2} \rightarrow 0$ using the proper nonperturbative light-cone wave functions. The factors $P^{T, L}$ turn out to be significantly reduced at small $Q^{2}[3]$.

\subsection{Coherence length for gluon shadowing}

Shadowing in the nuclear gluon distributing function at small $x_{B j}$ which looks like gluon fusion $G G \rightarrow G$ in the infinite momentum frame of the nucleus, should be treated in the rest frame of the nucleus as shadowing for the Fock components of the photon containing gluons. 
The lowest gluonic Fock component is the $|\bar{q} q G\rangle$. The coherence length relevant to shadowing depends according to (1) on the effective mass of the $|\bar{q} q G\rangle$ which should be expected to be heavier than that for a $|\bar{q} q\rangle$, and even more for higher Fock components. Correspondingly, the coherence length $\left\langle l_{c}^{G}\right\rangle$ should be shorter and a onset of gluon shadowing is expected at smaller $x_{B j}$ compared to quarks.

For the coherence length $l_{c}^{G}$ one can use the same Eq. (1), but with the effective mass,

$$
M_{\bar{q} q G}^{2}=\frac{k_{T}^{2}}{\alpha_{G}\left(1-\alpha_{G}\right)}+\frac{M_{\bar{q} q}^{2}}{1-\alpha_{G}},
$$

where $\alpha_{G}$ is the fraction of the photon momentum carried by the gluon, and $M_{\bar{q} q}$ is the effective mass of the $\bar{q} q$ pair. This formula is, however, valid only in the perturbative limit. It is apparently affected by the nonperturbative interaction of gluons which was found in [1] to be much stronger than for a $\bar{q} q$ pair. Since this interaction may substantially modify the effective mass $M_{\bar{q} q G}$ we switch to the formalism of Green function described above, which recovers Eq. (6) in the limit of high $Q^{2}$.

We treat gluons as massless and transverse. For factor $P$ defined in (1) one can write,

$$
\left\langle P^{G}\right\rangle=\frac{N^{G}}{D^{G}}
$$

where

$$
\begin{aligned}
N^{G}= & m_{N} x_{B j} \int d^{2} r_{1 G} d^{2} r_{1 q \bar{q}} d^{2} r_{2 G} d^{2} r_{2 q \bar{q}} d \alpha_{q} d \ln \left(\alpha_{G}\right) \tilde{\Psi}_{\bar{q} q G}^{\dagger}\left(\vec{r}_{2 G}, \vec{r}_{2 q \bar{q}}, \alpha_{q}, \alpha_{G}\right) \\
& \times\left(\int_{z_{1}}^{\infty} d z_{2} G_{\bar{q} q G}\left(\vec{r}_{2 G}, \vec{r}_{2 q \bar{q}}, z_{2} ; \vec{r}_{1 G}, \vec{r}_{1 q \bar{q}}, z_{1}\right)\right) \tilde{\Psi}_{\tilde{q} q G}\left(\vec{r}_{1 G}, \vec{r}_{1 q \bar{q}}, \alpha_{q}, \alpha_{G}\right) \\
D^{G} & =\int d^{2} r_{1 G} d^{2} r_{1 q \bar{q}} d^{2} r_{2 G} d^{2} r_{2 q \bar{q}} d \alpha_{q} d \ln \left(\alpha_{G}\right) \widetilde{\Psi}_{\tilde{q} q G}^{\dagger}\left(\vec{r}_{2 G}, \vec{r}_{2 q \bar{q}}, \alpha_{q}, \alpha_{G}\right) \\
& \times \delta^{(2)}\left(\vec{r}_{2 G}-\vec{r}_{1 G}\right) \delta^{(2)}\left(\vec{r}_{2 q \vec{q}}-\vec{r}_{1 q \bar{q}}\right) \tilde{\Psi}_{\bar{q} q G}\left(\vec{r}_{1 G}, \vec{r}_{1 q \bar{q}}, \alpha_{q}, \alpha_{G}\right)
\end{aligned}
$$

Here we have introduced the Jacobi variables, $\vec{r}_{q \bar{q}}=\vec{R}_{\bar{q}}-\vec{R}_{q}$ and $\vec{r}_{G}=\vec{R}_{G}-\left(\alpha_{\bar{q}} \vec{R}_{\bar{q}}+\right.$ $\left.\alpha_{q} \vec{R}_{q}\right) /\left(\alpha_{\bar{q}}+\alpha_{q}\right) . \vec{R}_{G, q, \bar{q}}$ are the position vectors of the gluon, the quark and the antiquark in the transverse plane and $\alpha_{G, q, \bar{q}}$ are the longitudinal momentum fractions.

The results of calculations described in [3] are plotted by dotted curve in Fig. 1. Factor $\left\langle P^{G}\right\rangle$ is substantial smaller than in the case of $\bar{q} q$ fluctuations. For this reason onset of gluon shadowing is delayed and happens only at $x_{B j}<0.01$ as one can see from Fig. 2 where nuclear suppression of gluons is plotted as function of $x_{B j}$ for a few nuclei [1] at $Q^{2}=4$ and $40 \mathrm{GeV}^{2}$.

\section{Exclusive electroproduction of vector mesons}

\subsection{Color transparency}

Exclusive diffractive vector meson production off nuclei by highly virtual photons first suggested in [4] is an effective way to search for the color transparency (CT) effects. This process 
avoids the usual trouble inherent for CT search in quasi-elastic $A\left(e, e^{\prime} p\right) X$ or $A(p, 2 p) X$ reactions where the particle energies are proportional to $Q^{2}$. On the one hand, the energy should be increased in order to freeze the small size fluctuations, on the other hand the cross section vanishes at large $Q^{2}$. This fact explains the failure to detect CT effects in experiments NE18 [5] at SLAC and [6] at BNL.

The light-cone dipole approach to (virtual) photoproduction of vector mesons was suggested in [7]. The cross section of coherent production reads,

$$
\frac{d \sigma_{c o h}\left(\gamma^{*} A \rightarrow V A\right)}{d^{2} q}=4\left|\int d^{2} b e^{i \vec{q} \vec{b}}\left\langle V\left|1-\exp \left[-\frac{1}{2} \sigma(r) T_{A}(b)\right]\right| \gamma^{*}\right\rangle\right|^{2} .
$$

Here the photoproduction amplitude is averaged over the transverse $\bar{q} q$ separation weighted by the product of the photon and vector meson light-cone wave functions. The coherence length is supposed to be much longer than the nuclear radius.

The nucleus to nucleon ratio of the incoherent quasi-elastic photoproduction cross sections has the form $[7,4]$,

$$
T_{A}\left(Q^{2}\right)=\frac{\sigma_{A}}{A \sigma_{p}}=\frac{1}{A} \int d^{2} \vec{b} T(b) \frac{\left\langle V\left|\sigma(r) \exp \left[-\frac{1}{2} \sigma(r) T(b)\right]\right| \gamma^{*}\right\rangle^{2}}{\left\langle V|\sigma(r)| \gamma^{*}\right\rangle^{2}}
$$

The E665 experiment at Fermilab [8] succeeded to detect the predicted CT effect in process $\gamma^{*} A \rightarrow \rho X$, both coherent and quasielastic. The observed $Q^{2}$ dependence of the exponent $\alpha\left(Q^{2}\right)$ for the cross section parameterized as $\propto A^{\alpha}$ is depicted in Fig. 4 together with the results of calculations using Eqs. (10), (11) [4]. The data well agree with the theoretical predictions.

Unfortunately, statistical confidence of the observed effect is rather low and new highstatistics measurements have been performed recently by the HERMES experiment [9]. However, as was predicted in [10] at medium energies CT effects can be easily imitated by the coherence length effects. Indeed, only variation of nuclear transparency related to the coherence length effect was observed in [9].

Another manifestation of CT would be observation of the illuminating nuclear effects expected for virtual photoproduction of radially excited vector mesons. It was first predicted in [7] that due to the nodal shape of the $2 S$ state wave functions, their production should be enhanced by nuclei. Indeed, the projection of the produced $\bar{q} q$ wave packet to the $2 S$ wave function is subject to a strong cancelation caused by the node. The mean transverse separation in the produced $\bar{q} q$ wave packet propagating through the nucleus shrinks due to the color filtering effect, therefore the projection to the $2 S$ state increases. This effect explains why hadroproduced $J / \Psi$ and $\Psi^{\prime}$ have nearly the same nuclear suppression in spite of the larger mean radius of $\Psi^{\prime}[11]$. The nodal structure of the $2 S$ states leads to a nontrivial behavior of nuclear transparency as function of $A$ and $Q^{2}[4]$.

Concluding, there is a strong demand for a new high-energy dedicated experiment for coherent and incoherent electroproduction of vector mesons off nuclei which could detect the CT effect with a better confidence. Measurements at the eRHIC collider will provide a 
unique opportunity to detect coherent process event by event without the incoherent background. CT makes the central region of the nucleus more transparent increasing the mean square radius of the coherent amplitude (10). Correspondingly, the positions of diffractive minima and maxima in the differential cross section (10) shift towards small $q$ and observation of this effect would be another test of CT.

\subsection{Quasielastic photoproduction of $J / \Psi$. Probing gluons at small $\boldsymbol{x}$ in nuclei}

Nuclear transparency (11) for photoproduction of a small size heavy quarkonium is quadratic in the dipole cross section,

$$
T_{A} \propto \frac{\langle\sigma(r, x)\rangle_{A}^{2}}{\langle\sigma(r, x)\rangle_{N}^{2}}
$$

The dipole cross section for small separations can be expressed in terms of the gluon density,

$$
\sigma(r, x) \approx \frac{\pi^{2}}{3} G(x) r^{2}
$$

where $G(x)=x g(x)$.

Thus, nuclear transparency $T_{A} \propto\left(G_{A}^{2}(x) / G_{N}^{2}(x)\right.$ is extremely sensitive to the gluon density at small $x$ in nuclei [12]. It should manifest itself as an additional suppression of the $J / \Psi$ production rate off nuclei compared to the standard mechanisms.

Nuclear transparency for photoproduction of $J / \Psi$ was measured in the NMC experiment [13]. They found a decreasing energy dependence of nuclear transparency in contradiction with predictions [14] based on the ideas of color transparency, but in a good agreement with predictions $[7,15]$ based of the effect of coherence length. However, the values of $x=\left(M_{\Psi}^{2}+Q^{2}\right) / s>0.02$ are too large for onset of gluon shadowing which is expected at $x<10^{-2}$ [1] (see Fig. 2). Extension of these measurements up to the eRHIC energy range would provide a direct access to the gluon density at small $x$ in nuclei.

\section{Nuclear enhancement of inclusive $J / \Psi$ production}

The EMC [16] and NMC [17] collaborations have observed an enhancement of the production rate of $J / \Psi$ off heavy nuclei, $\gamma A \rightarrow J / \Psi X$, at $x_{1}<0.85$ compared to a proton or a light nuclear target: the experimental ratio of the cross sections per nucleon on tin to carbon is above one by about $10 \%$ as depicted in Fig. 5 . If this enhancement is fully attributed to gluon antishadowing - as the authors of experiment [17] assume - the gluon density in heavy nuclei should be enhanced by about $40 \%$ since $J / \Psi$ must be suppressed by about $30 \%$ due to usual absorption effects. Such a strong enhancement of gluons in tin compared to carbon substantially exceeds any reasonable theoretical expectation.

A novel mechanism for diffractive inelastic photoproduction of $J / \Psi$ off nuclei is suggested in [18]. The Pomeron exchange can be split into two color-exchange interactions with different nucleons, keeping the beam and the nuclear remnants in the final state colorless. 
Thus, the $J / \Psi$ photoproduction on a nucleus can result from the photoproduction on a bound nucleon of a $c \bar{c}$ pair in a color-octet state which propagates through nuclear matter loosing energy via hadronization until another color-exchange interaction inside the nucleus converts the pair back to a colorless state. Actually, this is a specific QCD mechanism of diffractive excitation of the nucleus. The energy of the $J / \Psi$ (and the excitation energy of the nucleus) is less than the photon energy by the amount lost via hadronization by $c \bar{c}$ pair while it is in the color octet state. We assume a constant energy loss per unit of length $d E / d z=-\kappa$, what is true both for the color string [19] and for the bremsstrahlung [20] mechanisms of hadronization. In this case $x_{1}=1-\kappa \Delta z / \nu$, where $\Delta z$ is the longitudinal distance covered by the $c \bar{c}$ pair in the color-octet state.

The correction from this mechanism to the cross section for nuclear photoproduction can be calculated as follows

$$
\begin{aligned}
\Delta\left(\frac{d \sigma_{\Psi}^{\gamma A}}{d x_{1}}\right)= & 2 \pi \frac{\nu}{\kappa} B_{e l}^{\Psi N} \sigma(\gamma N \rightarrow J / \Psi N) \int d^{2} b \int_{-\infty}^{\infty} d z_{1} \rho_{A}\left(b, z_{1}\right) \int_{z_{1}}^{\infty} d z_{2} \rho_{A}\left(b, z_{2}\right) \\
& \delta\left[z_{2}-z_{1}-\left(1-x_{1}\right) \frac{\nu}{\kappa}\right] \exp \left\{-\sigma_{i n}^{\Psi N}\left[T(b)-T_{z_{1}}(b)+T_{z_{2}}(b)\right]\right\}
\end{aligned}
$$

The only unknown parameter we are left with in eq. (14) is $\kappa$, the energy loss per unit length. In the string model it equals the string tension. The color-octet string tension $\kappa$ may substantially exceed the known value for color-triplet strings $\kappa_{3} \approx\left(2 \pi \alpha_{R}^{\prime}\right)^{-1} \approx 1 \mathrm{GeV} / \mathrm{fm}$ [19], where $\alpha_{R}^{\prime} \approx 1 \mathrm{GeV}^{-2}$ is the universal slope of Regge trajectories. Indeed, the string tension of the color-octet string must to be related to the slope of the Pomeron trajectory, $\alpha_{P}^{\prime} \approx 0.2 \mathrm{GeV}^{-2}$, which is much smaller than $\alpha_{R}^{\prime}$. Thus, $\kappa_{8} \approx\left(2 \pi \alpha_{P}^{\prime}\right)^{-1} \approx 4 \mathrm{GeV} / \mathrm{fm}$.

One may also treat the energy loss perturbatively as a result of gluon bremsstrahlung by the color-octet $c \bar{c}$ pair. It was demonstrated in [20] that bremsstrahlung provides a constant density of energy loss, similar to the string model. The mean squared color-octet charge is $9 / 4$ times bigger than the color-triplet one, so is the energy loss. Besides, in the case under discussion the color-charge appears during the first interaction with a bound nucleon at the time $t=t_{1}$, then propagates for the time, $\Delta t=t_{2}-t_{1}$ and disappears when the $c \bar{c}$ pair becomes colorless. Such a process with switch-on and switch-off causes a double bremsstrahlung [20]: only that part of the frequency spectrum is emitted during a time interval $\Delta t$, which has radiation time $t_{T}=2 \omega / k_{T}^{2}<\Delta t$, where $\omega$ and $k_{T}$ are the energy and transverse momentum of the radiated gluon, respectively. At the time $t=t_{2}$ when the $c \bar{c}$ pair converts to a colorless state and the radiation process stops. A new radiation process caused by the charge stopping starts, and another piece of the gluon spectrum is radiated, identical to the one previously emitted. Thus, the intensity of radiation and the energy loss are twice as large as in a single-scattering process. Thus, we expect the energy loss for gluon bremsstrahlung in the double-scattering process with a color-octet intermediate state to be 4.5 times larger than that for radiation of a color-triplet charge, produced in a single scattering process. This estimate is very close to the one we found above in the string model. so we fix for further calculations $\kappa=5 \mathrm{GeV} / \mathrm{fm}$.

The new mechanism provides an $x_{1}$-dependence of the cross section quite different from the standard one. There is no cross section for $x_{1}<1-\kappa_{1} R_{1} / \nu$, since the amount of lost 
energy is restricted by the length of the path of the color-octet $c \bar{c}$ pair inside the nucleus. This contribution does not peak at $x_{1} \rightarrow 1$, but may have even a minimum due to a longer path of the colorless $c \bar{c}$ pair in nuclear matter.

The contribution of this mechanism may cause an $A$-dependence steeper than $A^{1}$, since there is more room for integration over longitudinal coordinates $z_{1}$ and $z_{2}$. For this reason the contribution of the mechanism under discussion, is important for heavy nuclei at moderately high $x_{1}$. We compare the contribution of Glauber-Gribov mechanism and the novel one given by eq. (14) to $J / \Psi$ photoproduction on lead at different photon energies in Fig. 4 . We see a strong energy variation of the latter contribution, which shifts with energy towards $x_{1}=1$. At the same time the energy dependence of nuclear effects provided by a variation of the coherence and formation lengths is so small that we plotted only one curve for the Glauber-Gribov mechanism at $\nu=100 \mathrm{GeV}$.

We see that both mechanisms the Glauber one and the two-step production are of the same order at $x_{1} \approx 0.6-0.7$. The novel mechanism proposed here does not display the Feynman scaling typical for the standard mechanism, but this contribution grows with energy at fixed $x_{1}$. This is easy to understand: the total contribution integrated over $x_{1}$ is energy independent, but the range of $1-x_{1}$ shrinks $\propto 1 / \nu$.

Combining the novel and the Glauber contributions we have,

$$
\frac{d \sigma_{\Psi}^{\gamma A}}{d x_{1}}=S_{\Psi}^{\gamma N} \frac{d \sigma_{\Psi}^{\gamma N}}{d x_{1}}+\Delta\left(\frac{d \sigma_{\Psi}^{\gamma A}}{d x_{1}}\right)
$$

in Fig. 5 for two photon energies $\nu=70$ and $100 \mathrm{GeV}$ typical for the NMC experiment in comparison with the data [17].

Concluding, a novel QCD mechanism of charmonium photoproduction is suggested, which is not included in the standard multiple-scattering theory and can only occur in a nuclear target. This mechanism arises as a natural consequence of the colored structure of the Pomeron: the projectile hadron (or a hadronic fluctuation of the photon) induces a color dipole in the target nucleus, leaving the nucleus in a colorless state. This is a specific way of diffractive excitation of the nucleus and inclusive production of leading hadrons.

As different from the Glauber model the new mechanism contribution is essentially energy-dependent and violates Feynman scaling. Due to steeper an A-dependence this mechanism successfully competes with Glauber one at moderate $x_{1}$ in the energy range of the EMC and NMC experiments and nicely explains the observed $[16,17]$ nuclear enhancement of the photoproduction cross section. This effect is expected to be squeezed towards $x_{1}=1$ and to vanish under the quasielastic peak at higher energies. Further experimental study of energy dependence of inelastic photoproduction of $J / \Psi$ off nuclei, crucial for understanding of the underlying mechanism, will become possible at eRHIC. 


\section{Inclusive production of hadrons in DIS off nuclei}

\subsection{Nuclear modification of the quark fragmentation function}

Nuclear targets may provide a unique opportunity to look at the early stage of hadronization at a distance of a few Fermi from the origin. The Lorentz time dilation considerably increases the duration of this process, proportionally to the initial quark momentum. The produced hadrons detected at macroscopic distances carry limited information about the hadronization dynamics. The most important details are hidden at the early stage. A quarkgluon system originated from DIS interacts with a medium while it propagates through the nucleus. This can bring forth precious information about the structure of the excited system and its space-time development. The questions, which can be answered in such an analysis are:

- How long does it take to produce a hadron?

- How does the produced colorless wave packet attenuate in a nucleus?

- Does a quark attenuate in nuclear matter?

Nuclear medium affects the momentum spectrum of the produced particles, and one can call the new distribution a modified fragmentation function $D_{e f f}^{A}\left(z_{h}, p_{T}\right)$. Usually the experimental results are presented in a simple form as a ratio of the nucleus-to-nucleon cross sections,

$$
R_{A}\left(z_{h}, p_{T}\right)=\frac{D_{e f f}^{A}\left(z_{h}, p_{T}\right)}{A D^{N}\left(z_{h}, p_{T}\right)} .
$$

It is known [20] that a parton originated from a hard process loses energy for gluon radiation in vacuum with a constant rate. This is due to finiteness of the mean radiation time,

$$
t_{c} \sim \frac{2 \nu}{k_{T}^{2}} \alpha(1-\alpha)
$$

where $\alpha$ is a fraction of the light-cone momentum of the quark carried by the gluon and $k_{T}$ is its transverse momentum. $E_{q}=\nu$ is the quark energy.

However, for the leading hadron production with fraction $z_{h}$ of the initial quark momentum energy conservation restricts the energy range of radiated gluons,

$$
\Delta E_{\text {rad }}(t)=\nu \int_{\Lambda^{2}}^{Q^{2}} d k_{T}^{2} \int_{0}^{1} d \alpha \alpha \frac{d n_{g}}{d k_{T}^{2} d \alpha}\left[1-\exp \left(-t / t_{c}\right)\right] \Theta\left(1-z_{h}-\alpha\right)
$$

where $d n_{g} / d k_{T}^{2} d \alpha$ is the gluon radiation spectrum [21] and $\Lambda^{2}$ is the soft cut off.

As a result, at long times the quark camnot radiate at all. Propagation without radiation is subject to the Sudakov's suppression,

$$
S\left(t, z_{h}, Q^{2}\right)=\exp \left\{-\frac{t \gamma}{2 \nu}\left[\int_{1^{2}}^{Q^{2}} d k_{T}^{2} \int_{1-z_{h}}^{1} \frac{d \alpha}{\alpha^{2}(1-\alpha)}\right]\right\}=\exp \left(-\tilde{\pi}_{g}(t)\right) .
$$


where $\gamma=4 \alpha_{s} / 3 \pi$ and $\bar{n}_{g}(t)$ is the number of gluons nonradiated over time interval $t$.

The Sudakov formfactor (19) substantially reduces the mean time interval of hadronization and the production time of the leading hadron (better to say, a colorless ejectile which does not lose energy anymore).

The following model is used for leading hadron production [22]. In the large $N_{c}$ limit each radiated gluon can be replaced by a $q \bar{q}$ pair, and the products of gluon bremsstrahlung can be seen as a system of color dipoles [23]. It is natural to assume that the leading hadron originates from the color dipole, which includes the leading quark and the antiquark from the last, fastest emitted gluon. This $q \bar{q}$ dipole is to be projected on the hadron wave function, $\Psi\left(\beta, l_{T}\right)$, where $\beta$ is the fractions of the hadron light-cone momentum carried by one of the quarks and $l_{T}$ is the relative transverse momentum of the quarks in hadron. The probability $W\left(t, z_{h}, \nu, Q^{2}\right)$ for production of a meson at time $t$ has the form,

$$
\begin{gathered}
W\left(z_{h}, t\right)=N \int_{0}^{1} \frac{d \alpha}{\alpha} \delta\left[z_{h}-\left(1-\frac{\alpha}{2}\right) \frac{E_{q}(t)}{\nu}\right] \int_{\Lambda^{2}}^{Q^{2}} \frac{d k_{T}^{2}}{k_{T}^{2}} \frac{\exp \left(-t / t_{c}\right)}{t_{c}} \\
\times \quad \int d l_{t}^{2} \delta\left[l_{T}^{2}-\frac{9}{16} k_{T}^{2}\right] \int_{0}^{1} d \beta \delta\left[\beta-\frac{\alpha}{2-\alpha}\right]\left|\Psi_{h}\left(\beta, l_{T}\right)\right|^{2} S\left(z_{h}, t, Q^{2}\right) .
\end{gathered}
$$

Here $\alpha$ is the fraction of the quark light-cone momentum carried by the gluon emitted at the time $t$ by the quark of energy $E_{q}(t)=\nu-\Delta E_{\text {rad }}(t)$. According to the mechanism of color neutralization explained above, the leading colorless $q \bar{q}$ pair is produced with an energy $E_{q}(t)(1-\alpha / 2)$. On the other hand, this must be the energy $z_{h} \nu$ of the produced hadron, what is provided by the first two $\delta$-functions in eq. (20). The transverse momenta $l_{T}$ of the quark and the antiquark within the color dipole are connected to $k_{T}$ by relation $l_{T}^{2}=9 k_{T}^{2} / 16$, while the fraction of the hadron light-cone momentum carried by the quark is $\beta(t)=\alpha(t) /[2-$ $\alpha(t)$ ]. This is why we need the two last $\delta$-functions in eq. (20). Normalization factor $N$ in (20) cancels in ratio (16), so we do not specify it.

An example of the probability distribution for the leading hadron production time $W(t)$ calculated with Eq. (20) is plotted in Fig. 7. The production time shrinks towards $z_{h} \rightarrow 1$ as was predicted long time ago [24].

We assume that the produced colorless hadronic configuration evolves to the final hadron propagating through the nucleus. We use the path-integral approach suggested in [7] for evolution and attenuation of such a wave packet. The resulting nuclear suppression predicted in [22] is depicted by dashed curve in Fig. 8. Preliminary data from the HERMES experiment well confirm the predicted nuclear effects.

In addition to vacuum gluon radiation, there is an additional energy loss induced by nuclear broadening of the quark transverse momentum via multiple soft interactions of the quark in the nuclear matter. On the contrary to the vacuum cnergy loss, the induced one is a quadratic function of time. We plugged the induced energy loss estimated in[26] into our calculations.

The results including the induced energy loss shown in Fig. 8 by solid curve are not much different from the dashed one indicating that the absorption in final state is a more 
important effect than induced energy loss. This observation is important for the so called jet quenching probe which is planned to use as a signature for creation of quark-gluon plasma in heavy ion collisions at RHIC. The probe is based on expected enhancement of energy loss in a dense matter, however the absorption effects leading to a stronger suppression are usually missed.

It is difficult to disentangle between the effects of induced energy loss and absorption using data at medium energies. However at higher energies the production time increases and the effect of absorption vanishes. Nuclear broadening of transverse momentum does not vanish, but increases with energy (see next subsection). Correspondingly, the energy loss rate of the quark also increases, and the total induced energy loss is proportional to the initial quark energy. Thus, we expect that the difference between the dashed and solid curves in Fig. 8 does not vanish at higher energies. While the dashed curve (only the effect of absorption) approaches $R_{A}=1$, the solid curve is always below. This nontrivial expectation can be tested at eRHIC.

\subsection{Nuclear broadening of the transverse momentum of the quark}

A high-energy parton propagating through a medium experiences multiple interactions which increase its transverse momentum. Such a broadening of the transverse momentum can be measured in DIS on a nuclear target, which also includes the Fermi momentum of the participating nucleon. Although the available data from the EMC experiment [27] do not show any significant effect, this is related to a suppression by a factor $z_{h}^{2}$, where $z_{h}$ is the fraction of the quark momentum carried by the produced hadron. In this experiment $\left\langle z_{h}^{2}\right\rangle \sim 0.06$.

The effect of broadening has been treated as color filtering effect using the dipole representation in $[29,30,31]$. The general expression for the transverse momentum distribution of a quark after it propagated through nuclear medium of thickness $T_{A}=\int d z \rho_{A}(z)$ is derived recently in [31],

$$
\frac{d N_{q}}{d^{2} k_{T}}=\int d^{2} b d^{2} b^{\prime} \exp \left[i \vec{k}_{T}\left(\vec{b}-\vec{b}^{\prime}\right)\right] \Omega_{i n}^{q}\left(\vec{b}, \vec{b}^{\prime}\right) \exp \left[-\frac{1}{2} \sigma_{\bar{q} q}\left(\vec{b}-\vec{b}^{\prime}, E_{q}\right) T_{A}\right],
$$

characterizing the initial transverse momentum distribution of the quark originated from DIS; $\sigma_{\tilde{q} q}\left(r_{T}, E_{q}\right)$ is the total cross section of interaction of a $\bar{q} q$ dipole with a nucleon. It rises with energy $E_{q}$ of the quark.

Broadening of the mean transverse momentum squared turns out to be independent of the initial $k_{T}$ distribution and is proportional to the nuclear thickness,

$$
\Delta\left\langle k_{T}^{2}\right\rangle=2 C(0, s)\left\langle T_{A}\right\rangle
$$

where the dipole cross section is represented as

$$
\sigma_{\bar{q} q}\left(r_{T}, s\right)=C\left(r_{T}, s\right) r_{T}^{2}
$$

The results of calculations of $C(0, s)$ performed in [31] based on realistic phenomonological results for the dipole cross section [2,1], and corrected for gluon shadowing are depicted 
in Fig. 9. They overestimate the available data for Drell-Yan process [32]. There is a strong demand for new measurements of transverse momentum broadening and in a different process. Future data from eRHIC for inclusive hadron production in DIS off nuclei should provide new precious information.

\section{References}

[1] B.Z. Kopeliovich, A. Schäfer and A.B. Tarasov, Phys. Rev. D62 (2000) 054022

[2] K. Golec-Biernat and M. Wüsthoff, Phys. Rev. D53 (1999) 014017; hep-ph/9903358.

[3] B.Z. Kopeliovich, J. Raufeisen, A.V. Tarasov, Nuclear Shadowing and Coherence Length for Longitudinal and Transverse Photons, hep-ph/0003136

[4] B.Z. Kopeliovich, J. Nemchick, N.N. Nikolaev, B.G. Zakharov, Phys. Lett. B309 (1993) 179; Phys. Lett. B324 (1994) 469

[5] The NE18 Collaboration, N.C.R. Makins et al., Phys. Rev. Lett. 72 (1994) 1986

[6] A.S. Carroll et al., Phys. Rev. Lett. 61 (1988) 1698

[7] B.Z. Kopeliovich and B.G. Zakharov, Phys. Rev. D44 (1991) 3466.

[8] E665 Collaboration, M.R. Adams et al., Phys. Rev. Lett. 74 (1995) 1525

[9] K. Ackerstaff et al. (The HERMES Collaboration), Phys. Lett. B475 (2000) 386

[10] J. Hüfner, B.Z. Kopeliovich and J. Nemchik, Phys. Lett. B383 (1996) 362

[11] J. Hüfner and B.Z. Kopeliovich, Phys. Rev. Lett. 76 (1996) 192

[12] M.G. Ryskin, Z. Phys. C57 (1993) 89

[13] NMC Collaboration, M. Arneodo et al., Phys. Lett. B332 (1994) 195

[14] G.N. Farrar, L.L. Frankfurt and M.I. Strikman, Phys. Rev. Lett. 64 (1990) 2996

[15] O. Benhar, B.Z. Kopeliovich, Ch. Mariotti, N.N. Nikolaev and B.G. Zakharov, Phys.Rev.Lett. 69 (1992) 1156.

[16] The EM Collaboration, J.J. Aubert et al., Phys. Lett. 152B (1985) 433

[17] The NM Collaboration, P. Amaudruz et al., Nucl. Phys. B 371 (1992) 553

[18] J. Hüfner, B.Z. Kopeliovich and Al.B. Zamolodchikov, Z. Phys. A357 (1997) 113

[19] A. Casher, H. Neubereger and S. Nussinov, Phys. Rev. D20 (1979) 179.

[20] F. Niedermayer, Phys. Rev. D34 (1986) 3494. 
[21] J.F. Gunion and G. Bertsch, Phys. Rev. D25 (1982) 746

[22] B.Z. Kopeliovich, J. Nemchik, E. Predazzi, Hadronization in Nuclear Environment, nucl-th/9607036

[23] A.H. Mueller, Nucl. Phys. B415 (1994) 373

$[24]$

[25] B.Z. Kopeliovich and F. Niedermayer, Sov. J. Nucl. Phys. 42(1985) 504

[26] B. Baier, D. Schiff and B.G. Zakharhov, hep-ph/0002198

[27] J. Ashman et al., Z. Phys. C52 (1991) 1

[28] V. Muccifora, in: Proc. of the 38th International Winter Meeting on Nuclear Physics, Bormio, 24-29 Jan 2000, Italy (2000)

[29] J. Dolejši, J. Hüfner and B.Z. Kopeliovich, Phys.Lett. B312 (1993) 235

[30] U.A. Wiedemann and M. Gyulassy, Nucl. Phys. B560 (1999) 345

[31] M.B. Johnson, B.Z. Kopeliovich, A.V. Tarasov, Broadening of Transverse Momentum of Partons Propagating through a Medium, hep-ph/0006326

[32] P.L. McGaughey, J.M. Moss and J.C. Peng, Ann. Rev. Nucl. Part. Sci. 49 (1999) 217; hep-ph/9905447 


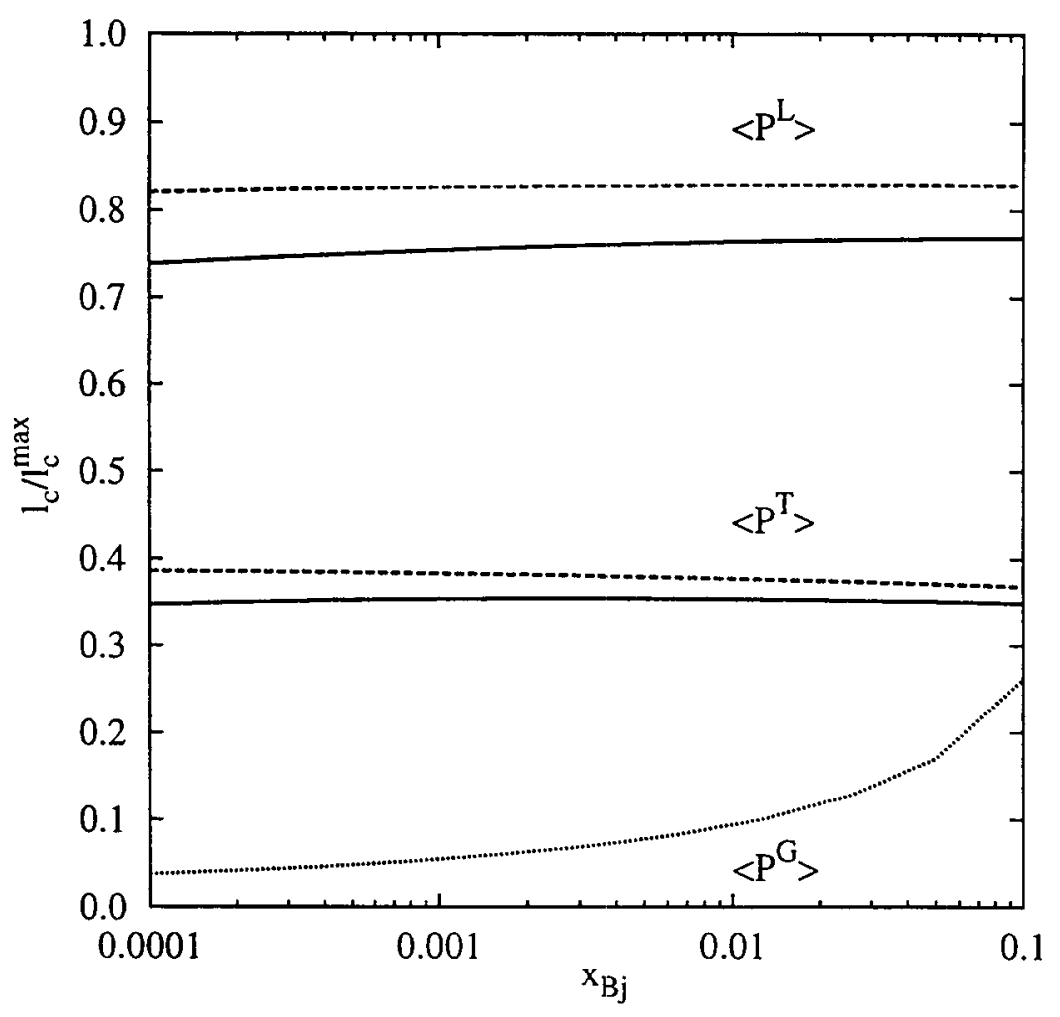

Figure 1: $x_{B j}$ dependence of the factor $\left\langle P^{T, L}\right\rangle$ and $\left\langle P^{G}\right\rangle$ defined in (1) corresponding to the coherence length for shadowing of transverse and longitudinal photons and gluon shadowing, respectively. Solid and dashed curves correspond to $Q^{2}=4$ and $40 \mathrm{GeV}^{2}$. 


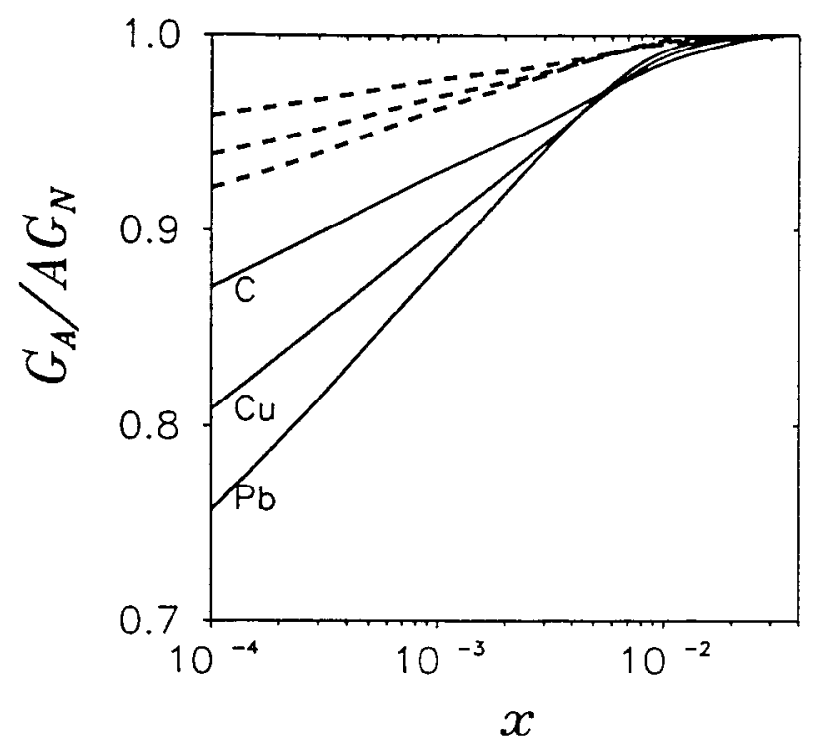

Figure 2: Ratio of the gluon distribution functions in nuclei (carbon, copper and lead) and nucleons at small Bjorken $x$ and $Q^{2}=4 \mathrm{GeV}^{2}$ (solid curves) and $40 \mathrm{GeV}^{2}$ (dashed curves).

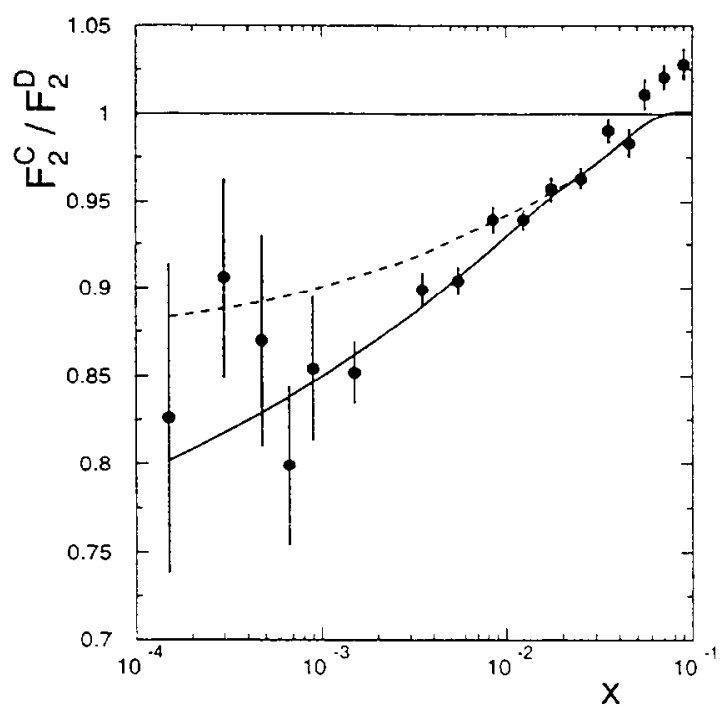

Figure 3: Normalized ratio of carbon to deuterium structure functions. The data are from the NMC experiment [17]. The dashed curve is calculated for the $\bar{q} q$ Fock component of the photon for each data point at the same values of $x$ and $Q^{2}$. The solid curve includes also the effect of gluon shadowing as it is calculated in [1]. 


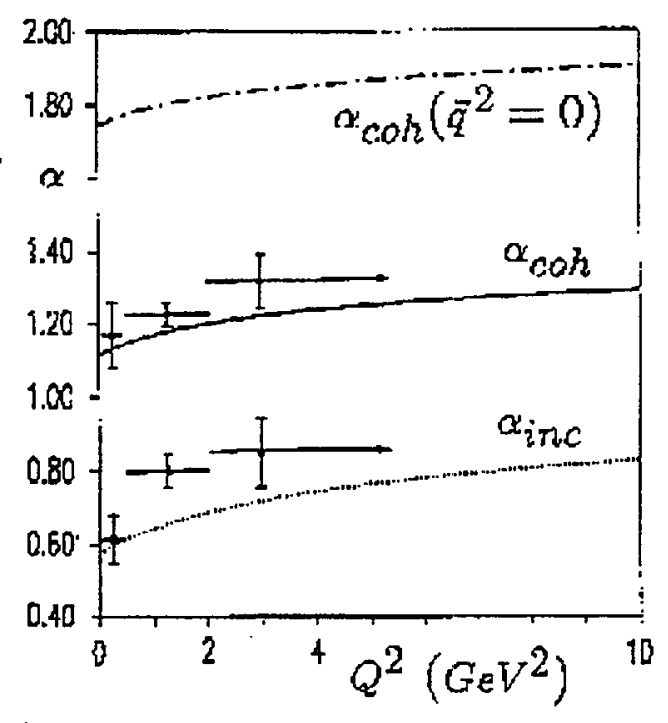

Fig.4

Figure 4: Predictions [4] for the $Q^{2}$ dependence of exponents of parametrizations $\sigma_{A}($ inc $) \propto A^{\alpha_{i n c}}, \quad\left[d \sigma_{A}(\text { coh }) / d q^{2}\right]_{\vec{q}^{2}=0} \propto A^{\alpha_{c o h}\left(\vec{q}^{2}=0\right)}$ and $\sigma_{A}(c o h) \propto A^{\alpha_{c o h}}$ vs. the E665 data [8].

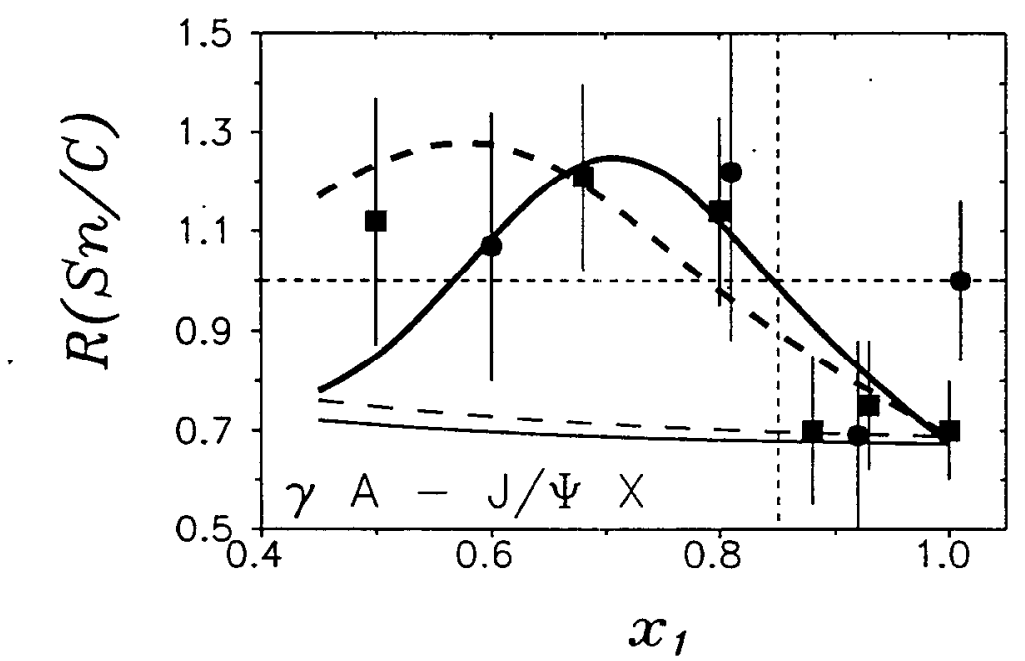

Figure 5: The data from the NMC experiment [17] for the ratio of inelastic $J / \Psi$ photoproduction rates on tin to carbon, measured with $200 \mathrm{GeV}$ (squares) and $280 \mathrm{GeV}$ (circles) muons. The thin solid and dashed curves correspond to the Glauber-Gribov mechanism for nuclear suppression of $J / \Psi$ and $\nu=100$ and $70 \mathrm{GeV}$, respectively. The thick solid and dashed curves show predictions based on the proposed novel mechanism of diffractive interaction with a nucleus at $\nu=100$ and $70 \mathrm{GeV}$, respectively. 


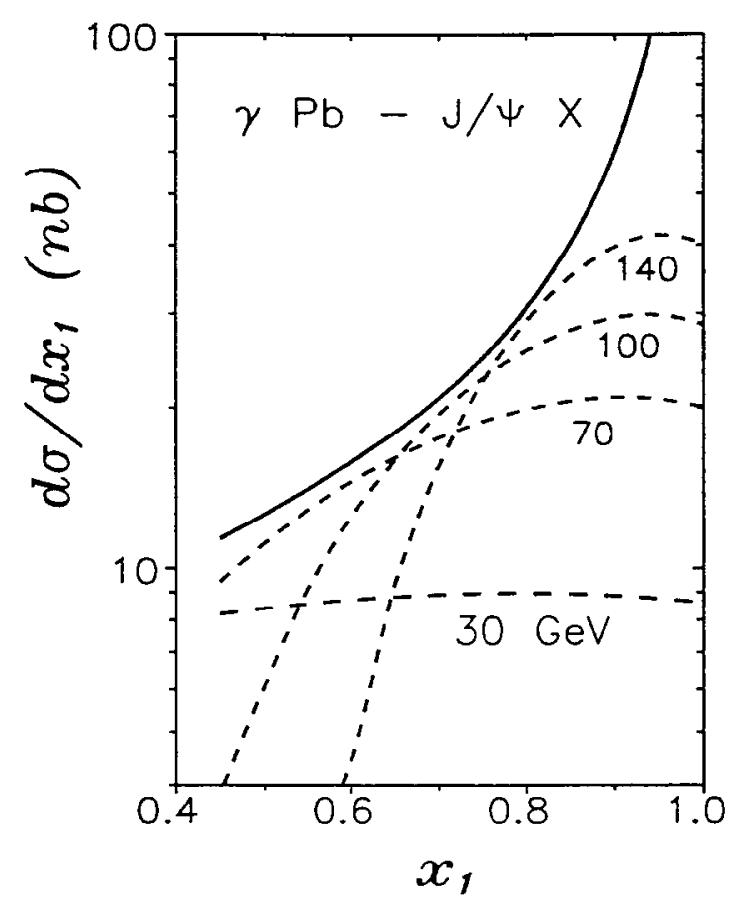

Figure 6: The cross section per nucleon for $J / \Psi$ photoproduction on lead. The solid curve corresponds to Glauber-Gribov mechanism of nuclear shadowing at $\nu=100 \mathrm{GeV}$. The novel mechanism contributions at $\nu=70,100$ and $140 \mathrm{GeV}$ are shown by dashed curves.

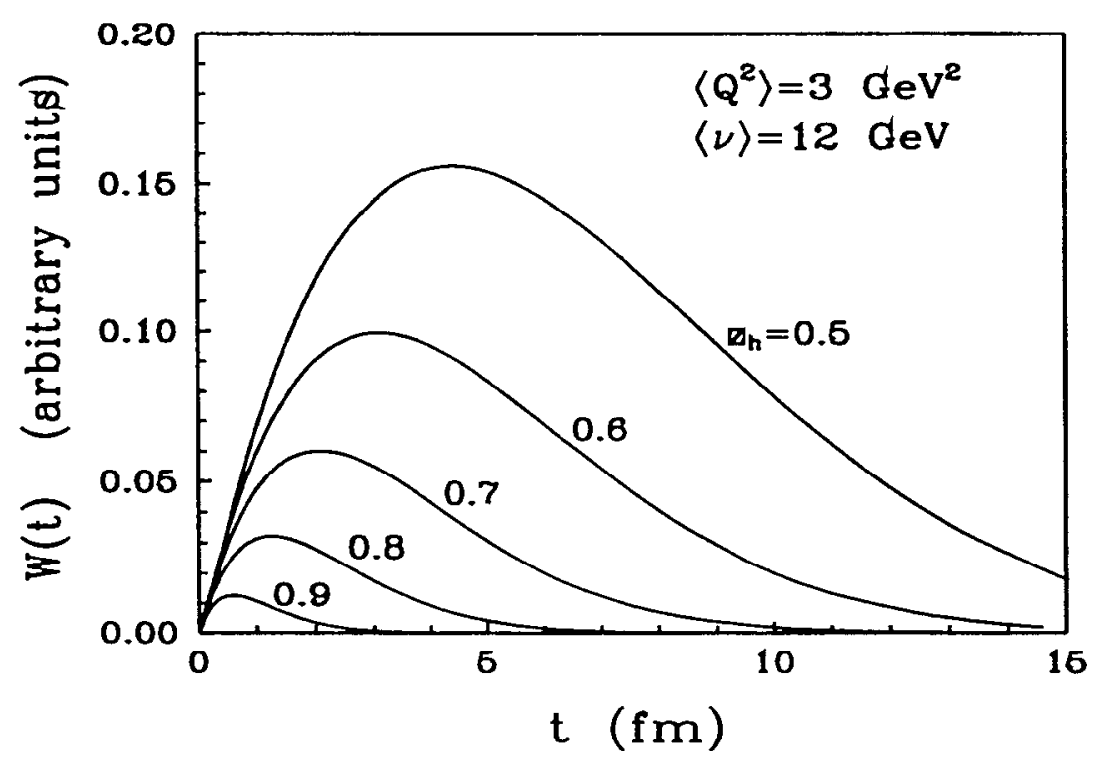

Figure 7: Distribution of the hadron production time at $\nu=12 \mathrm{GeV}$, $Q^{2}=3 \mathrm{GeV}^{2}$ and $z_{h}=0.5-0.9$ 


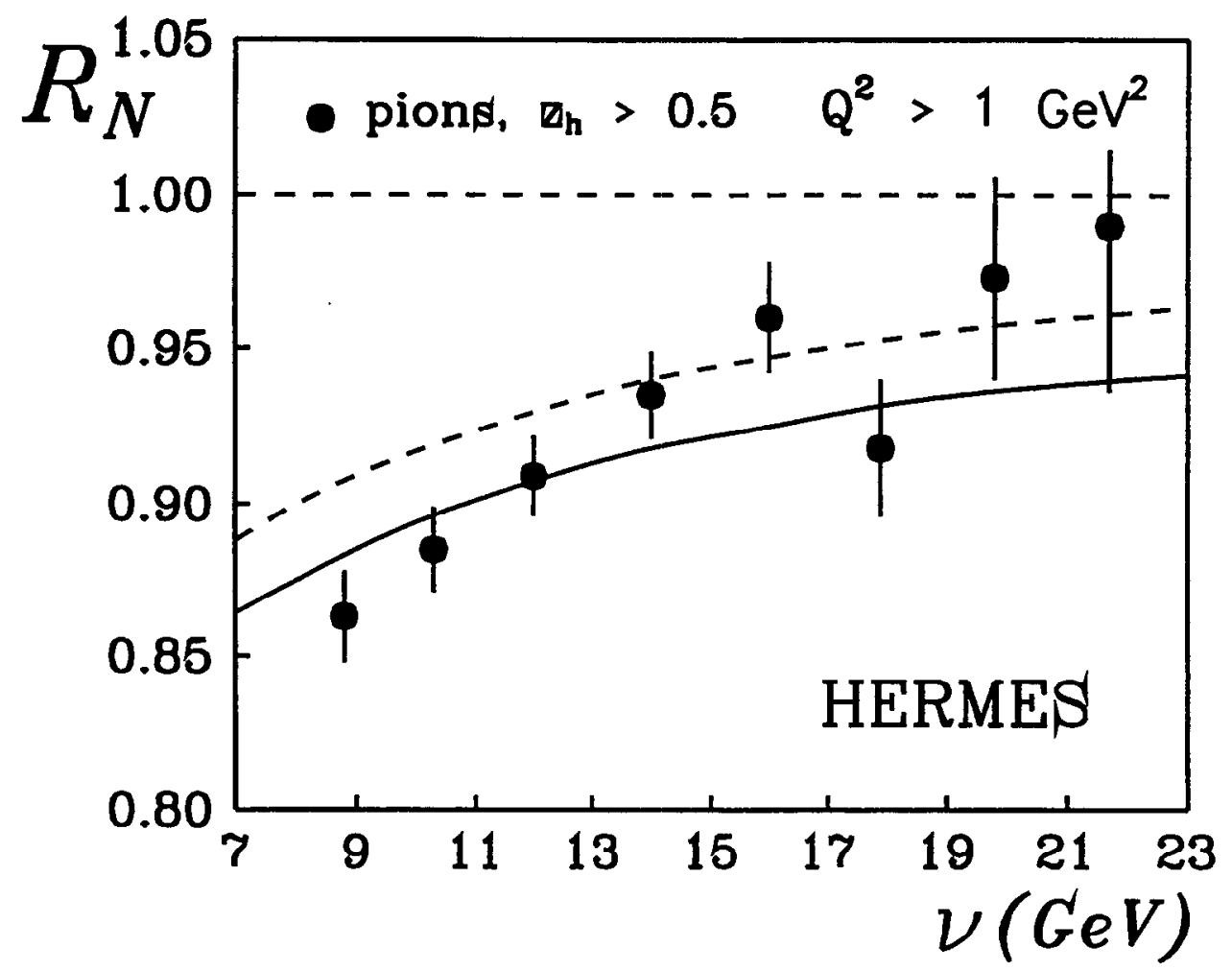

Figure 8: Comparison of the model calculations for the $\nu$ - dependence of nuclear suppression $R_{N^{14}}$ (16) integrated over $z_{h}$ with the HERMES data [28]. The solid and dashed curve are the model predictions for nuclear suppression with and without induced radiation respectively. 


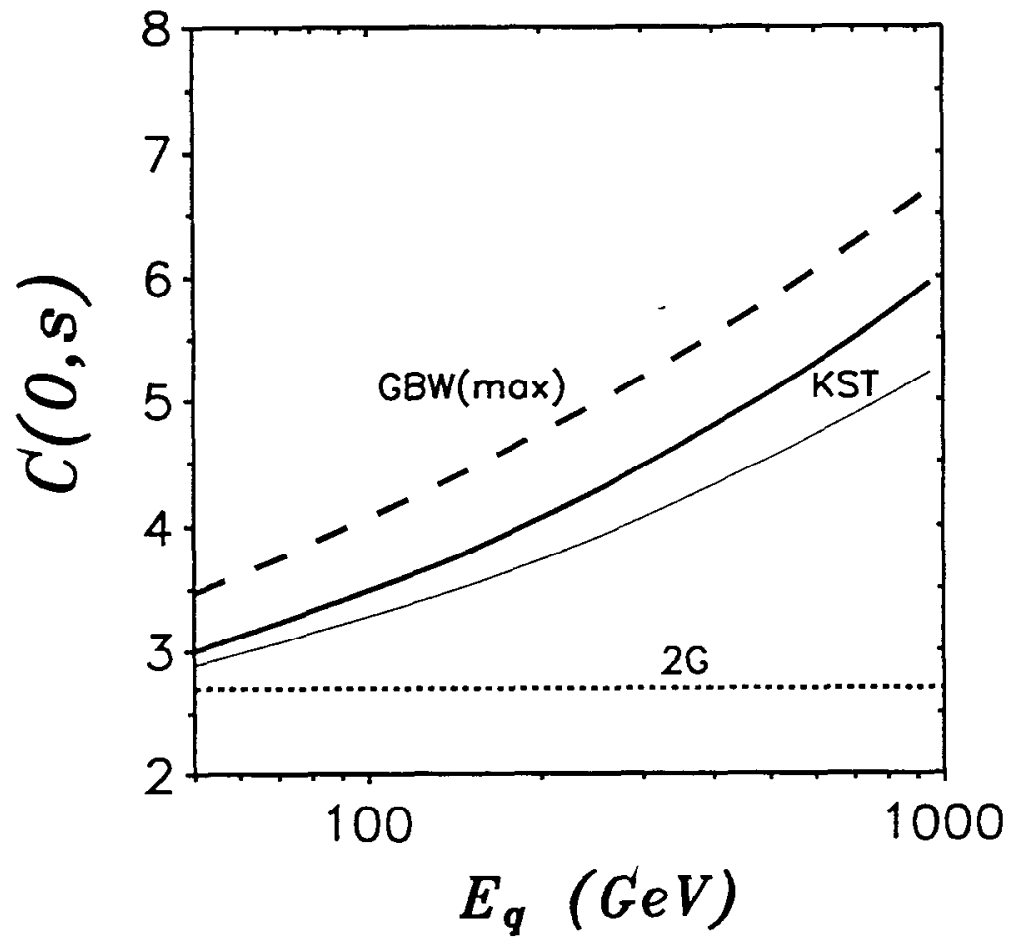

Figure 9: Factor $C(0, s)$ defined in (23) as function of the quark energy. The dashed and solid curves correspond to the models [2] and [1] respectively. The thin solid curve is the [1] curve corrected for gluon shadowing in lead. 


\title{
Anti-Quarks in Nucleons and Nuclei-The Role of Virtual
} Mesons

\author{
BNL June 2000 \\ Gerry Garvey \\ Los Alamos Nat. Lab \\ USA
}




\section{ABSTRACT}

\section{Antiquarks and Virtual Mesons in Nucleons and Nuclei}

Recent measurements, using $800 \mathrm{GeV}$ protons from the Fermilab Tevatron, of the anti-quark distributions in the proton and neutron provide compelling evidence that virtual pions play a large role in the quark-anti quark sea of the nucleon. This finding was contrary to a commonly held belief that the sea of the neutron and proton are nearly identical, but is quite consistent with earlier models of the nucleon. This observation that pions make up a large fraction of the nucleon sea makes it even more difficult to explain the constancy of the number of anti-quarks/nucleon as a function of nucleon number. 


\section{Partons in the Nucleon}

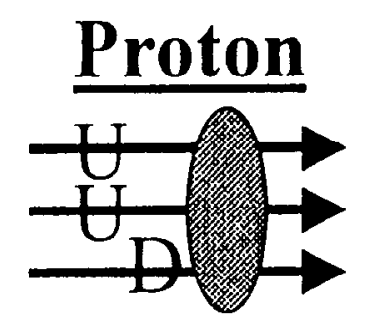

$\mathrm{Q}^{2}<1 \mathrm{Gev}^{2}$
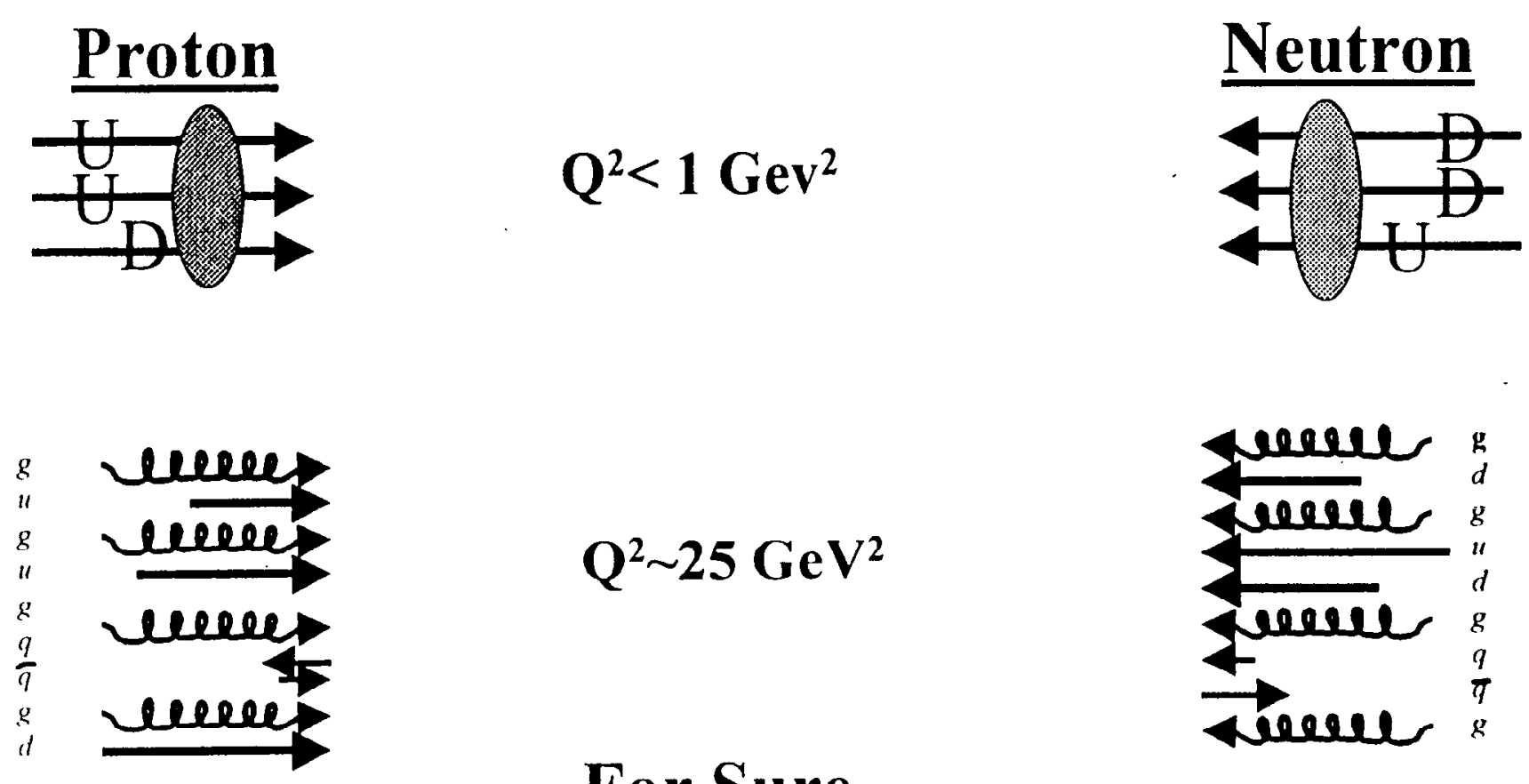

$\mathrm{Q}^{2} \sim 25 \mathrm{GeV}^{2}$

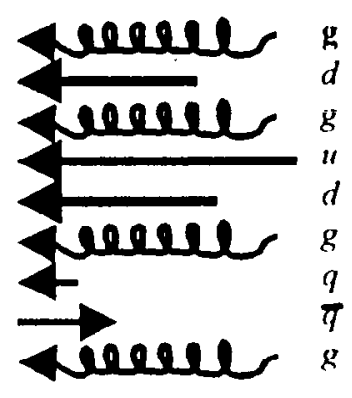

For Sure

$$
\begin{aligned}
& \int_{0}^{1}\left[u_{p}\left(x, Q^{2}\right)-\bar{u}_{p}\left(x, Q^{2}\right)\right] d x=\int_{0}^{1}\left[d_{n}\left(x, Q^{2}\right)-\bar{d}_{n}\left(x, Q^{2}\right)\right] d x=2 \\
& \int_{0}^{1}\left[d_{p}\left(x, Q^{2}\right)-\bar{d}_{p}\left(x, Q^{2}\right)\right] d x=\int_{0}^{1}\left[u_{n}\left(x, Q^{2}\right)-\bar{u}_{n}\left(x, Q^{2}\right)\right] d x=1 \\
& \text { What About? } \\
& \int_{0}^{1}\left[\bar{u}_{p}\left(x, Q^{2}\right)-\bar{d}_{p}\left(x, Q^{2}\right)\right] d x=? ?
\end{aligned}
$$

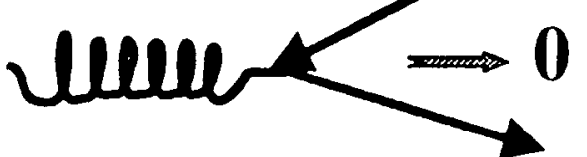




\section{Pair Production by Gluons}

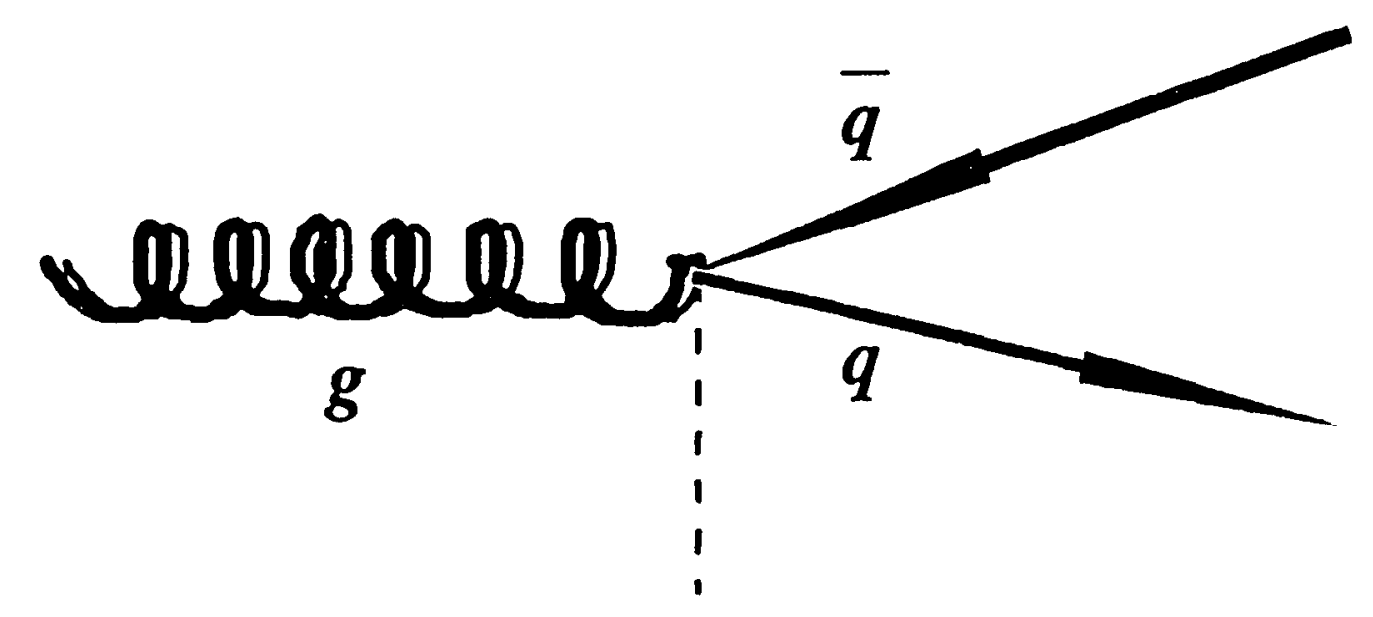

$M_{\mathrm{u}} \sim 2 M e V$

$M_{d} \sim 4 M e V$

Confinement scale $10^{-13} \mathrm{~cm}, \mathrm{p} \sim 200 \mathrm{MeV} / \mathrm{c}$

$$
\bar{u}(x)=\bar{d}(x) \text { to }<1 \%
$$




\section{Using Drell-Yan to Measure $\bar{d} / \bar{u}$}

$$
\begin{gathered}
p+p \rightarrow \gamma_{v}+X \rightarrow \mu^{+} \mu^{-}+X \\
x_{F}=p_{\|}^{\gamma} / p_{\|}^{\gamma, \max }=x_{1}-x_{2} \\
x_{1} x_{2} s=M_{\gamma}^{2} \\
\frac{d \sigma}{d x_{1} d x_{2}}=\frac{4 \pi \alpha^{2}}{9 s x_{1} x_{2}} \sum_{i} e_{i}^{2}\left(q_{i}^{b}\left(x_{1}, Q^{2}\right) \bar{q}_{i}^{t}\left(x_{2}, Q^{2}\right)+\bar{q}_{i}^{b}\left(x_{1}, Q^{2}\right) q_{i}^{t}\left(x_{2}, Q^{\prime}\right.\right.
\end{gathered}
$$

\section{Assumptions and Technique}

- No nuclear effects in deuterium.

- Charge symmetry $\bar{u}_{p}=\bar{d}_{n}, u_{p}=d_{n}$ etc.

- Choose kinematics with $x_{1}$ in valence dominated region of proton.

- To extract $\bar{d} / \bar{u}$ take $u_{v a l}, d_{v a l}, \bar{u}_{p}+\bar{d}_{p}, s, c$, from global fits: CTEQ, MRS.

$$
\begin{gathered}
\left.\frac{\sigma^{p d}}{2 \sigma^{p p}}\right|_{x_{1}>>x_{2}} \approx \frac{1}{2} \frac{\left(1+\frac{d\left(x_{1}\right)}{4 u\left(x_{1}\right)}\right)}{\left(1+\frac{d\left(x_{1}\right) \bar{d}\left(x_{2}\right)}{4 u\left(x_{1}\right) \bar{u}\left(x_{2}\right)}\right)}\left(1+\frac{\bar{d}\left(x_{2}\right)}{\bar{u}\left(x_{2}\right)}\right) \\
\frac{\frac{1}{A_{X}} \frac{d \sigma}{d x_{1} d x_{2}}}{\frac{1}{A_{Y}} \frac{d \sigma}{d x_{1} d x_{2}}} \approx \frac{\bar{u}^{X}\left(x_{2}\right)}{\bar{u}^{Y}\left(x_{2}\right)} .
\end{gathered}
$$


- [NC ᄂOUUOEV/C!

$\angle R A \equiv(4 C !)$

a E605 ( $80060 \%$ )

NLO calculatrons

$$
p A \rightarrow \mu^{+} \mu^{-} X
$$$$
\text { (GTEQ } 4 \mathrm{~m}, \mathrm{k}=1 \text { ) }
$$

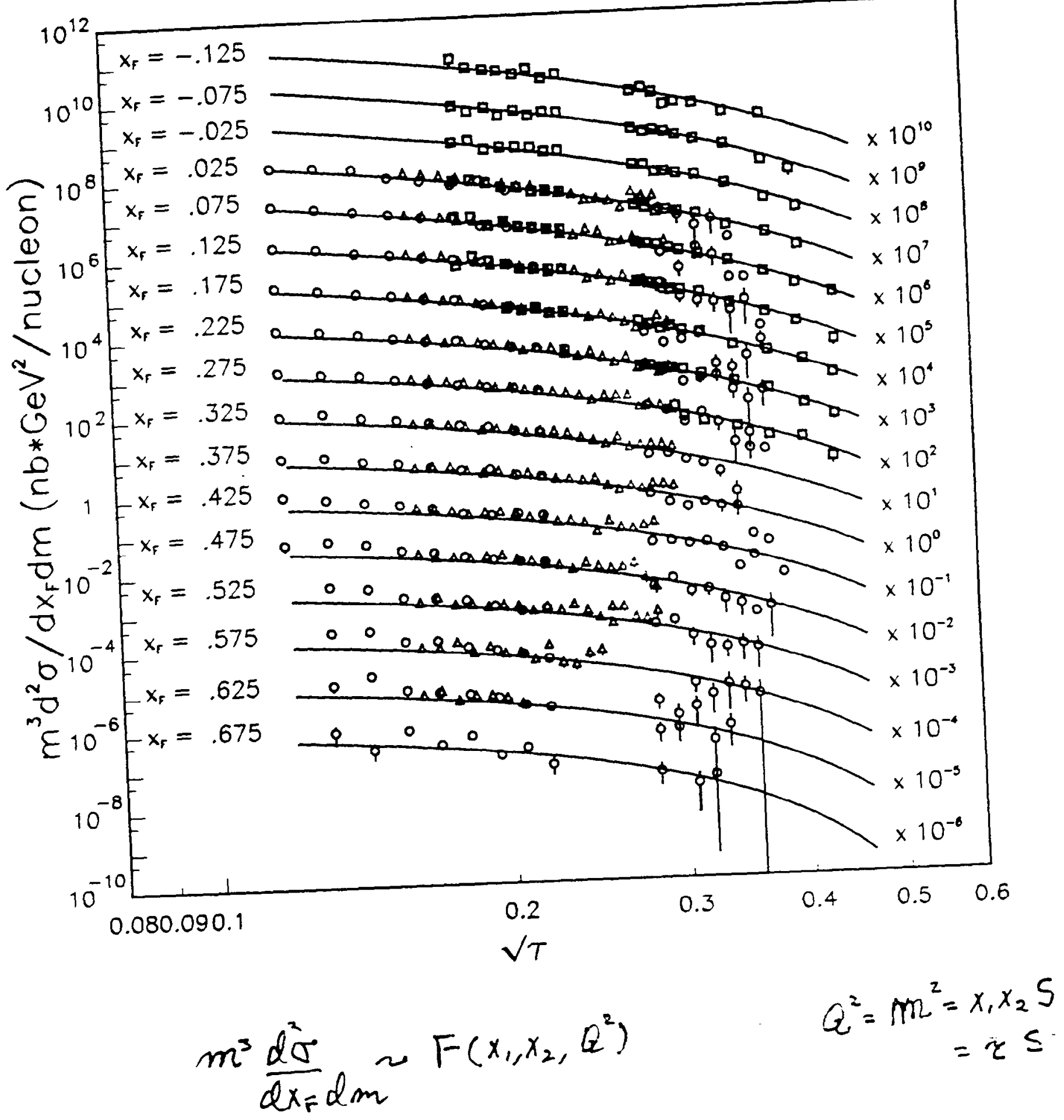

98 


\section{Abilene Christian University}

Donald Isenhower, Mike Sadler, Rusty Towell, Josh Bush, Josh Willis, Derek Wise

\section{Argonne National Laboratory}

Don Geesaman, Sheldon Kaufman, Naomi Makins,

Bryon Mueller, Ben Zeidman

Fermi National Accelerator Laboratory

Chuck Brown, Bill Cooper

Georgia State University

Gus Petitt, Xiao-chun He, Bill Lee

Illinois Institute of Technology

Dan Kaplan

Los Alamos National Laboratory

Melynda Brooks, Tom Carey, Gerry Garvey, Dave Lee, Mike Leitch, Pat McGaughey, Joel Moss, Brent Park, Jen-Chieh Peng, Andrea Palorırcek, Paul E. Reimer,

Walt Sondheim, Ne.il Thompson

Louisiana State University

Paul Kirk, Ying-Chao Wang, Zhi-Fu Wang

New Mexico State University

Mike Beddo, Ting Chang, Gary Kyle,

Vassilios Papavassiliou, J. Seldon, Jason Webb

Oak Ridge National Laboratory

Terry Awes, Paul Stankus, Glenn Young

Texas A \& M University

Carl Gagliardi, Bob Tribble, Eric Hawker, Maxim Vasiliev

Valparaiso University

Don Koetke, Paul Nord 


\section{FNAL E866 Spectrometer}

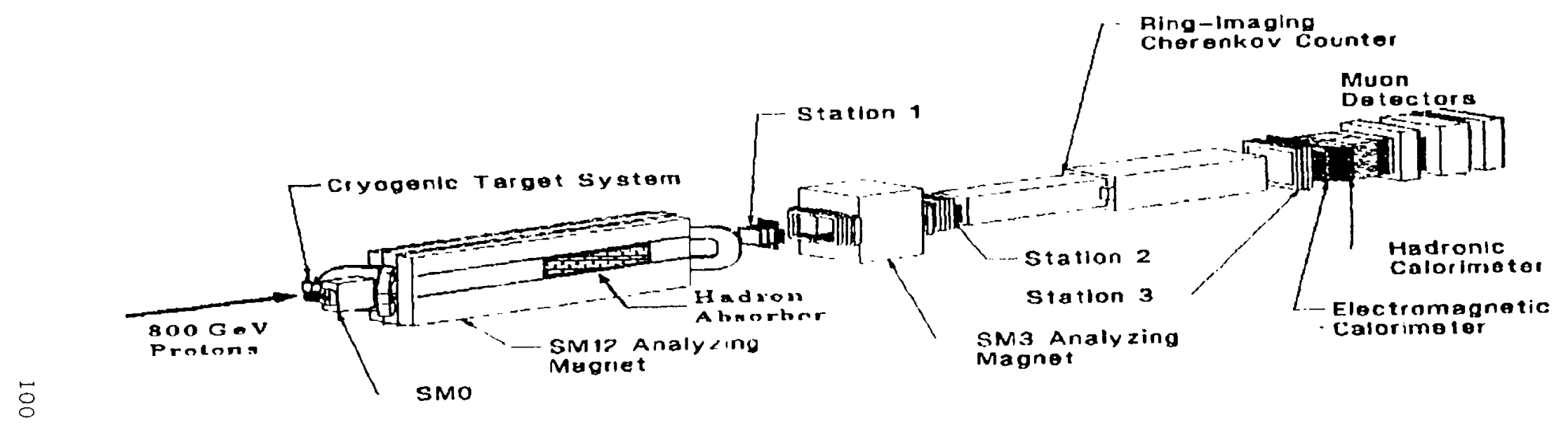

Scale: $60 \mathrm{~m}$ long, $3 \times 3 \mathrm{~m}^{2}$ at the back

$.50 \mathrm{~cm}$ long, Liquid H,D and empty targets

.Two acceptance defining magnets, SM0, SM12

- Thick $(-6 \mathrm{~m})$ hadron adsorber and beam stop in SM12, $\mu$ 's tranmitted

.4 trigger-tracking stations

Momentum analyzing magnet; SM3 


\section{Dimuon distributions}
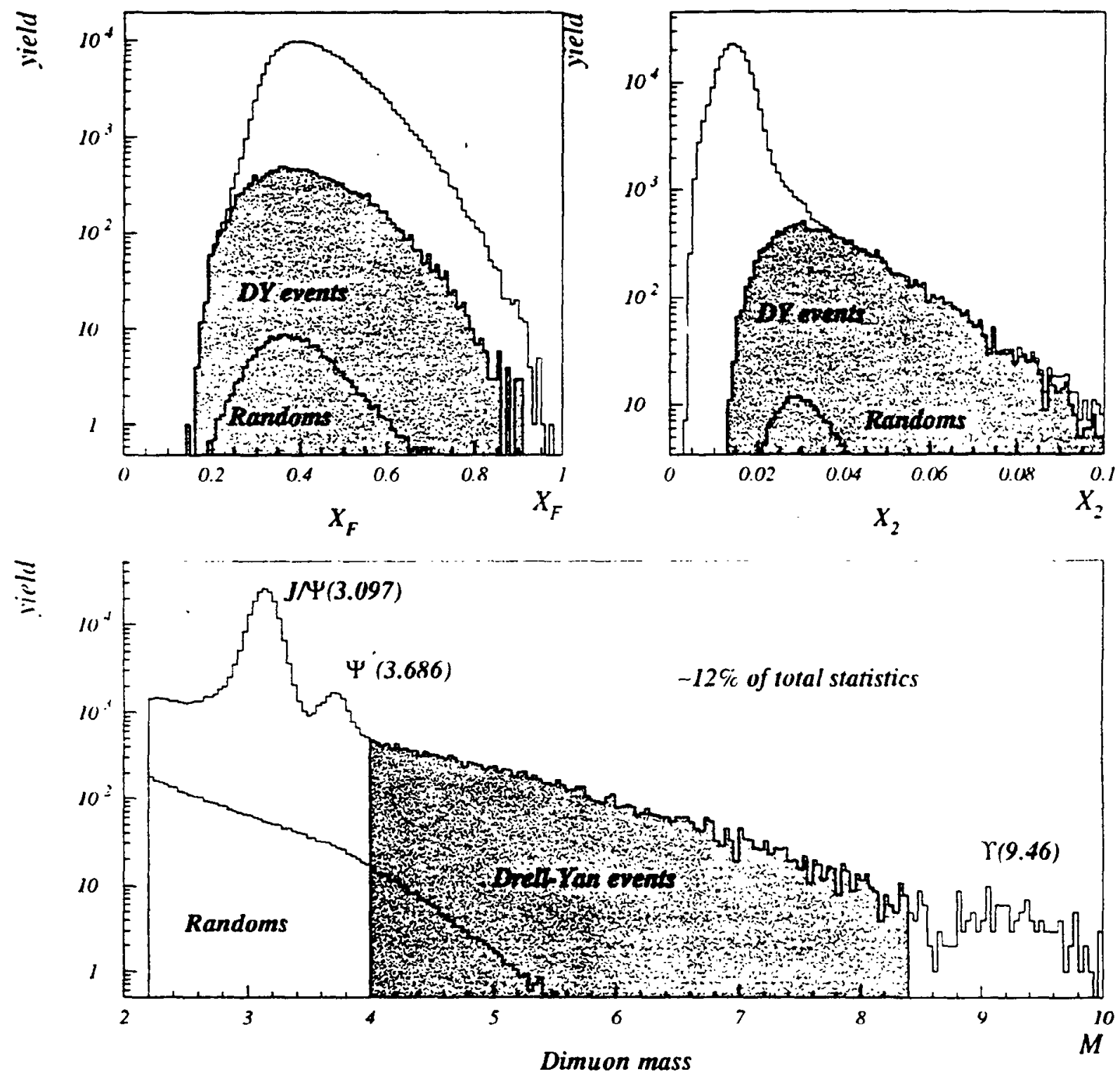

Over 130,000 Drell-Yan Pairs

Random Pairs: 9\% Empty Target Yield: 3\% Overall Normalization Uncertainty: $1 \%$ 


\section{Summary of Systematic Errors}

\section{Source}

1) Target thickness: $\frac{\rho_{\mathrm{p}} \mathrm{L}_{\mathrm{p}}}{\rho_{\mathrm{d}} \mathrm{L}_{\mathrm{d}}}$

\% Error

2) Flux: $\frac{N_{p}^{p} T_{p}^{p} \tau_{p}}{N_{p}^{d} T_{p}^{d} \tau_{d}}$

0.12

0.29

3) Detection Efficiency: $\frac{\Omega_{p} \varepsilon_{p}}{\Omega_{d} \varepsilon_{d}}$

0.10

4) Backgrounds:

Accidentals

0.20

Target Windows

0.17

Secondaries

$\underline{0.20}$

Total (1)-4) )

0.47

5) Rate Dependence of Event

0.80

Reconstruction

Total Systematic Error in High Mass Measurement

0.90 
E866 PRELIMINARY

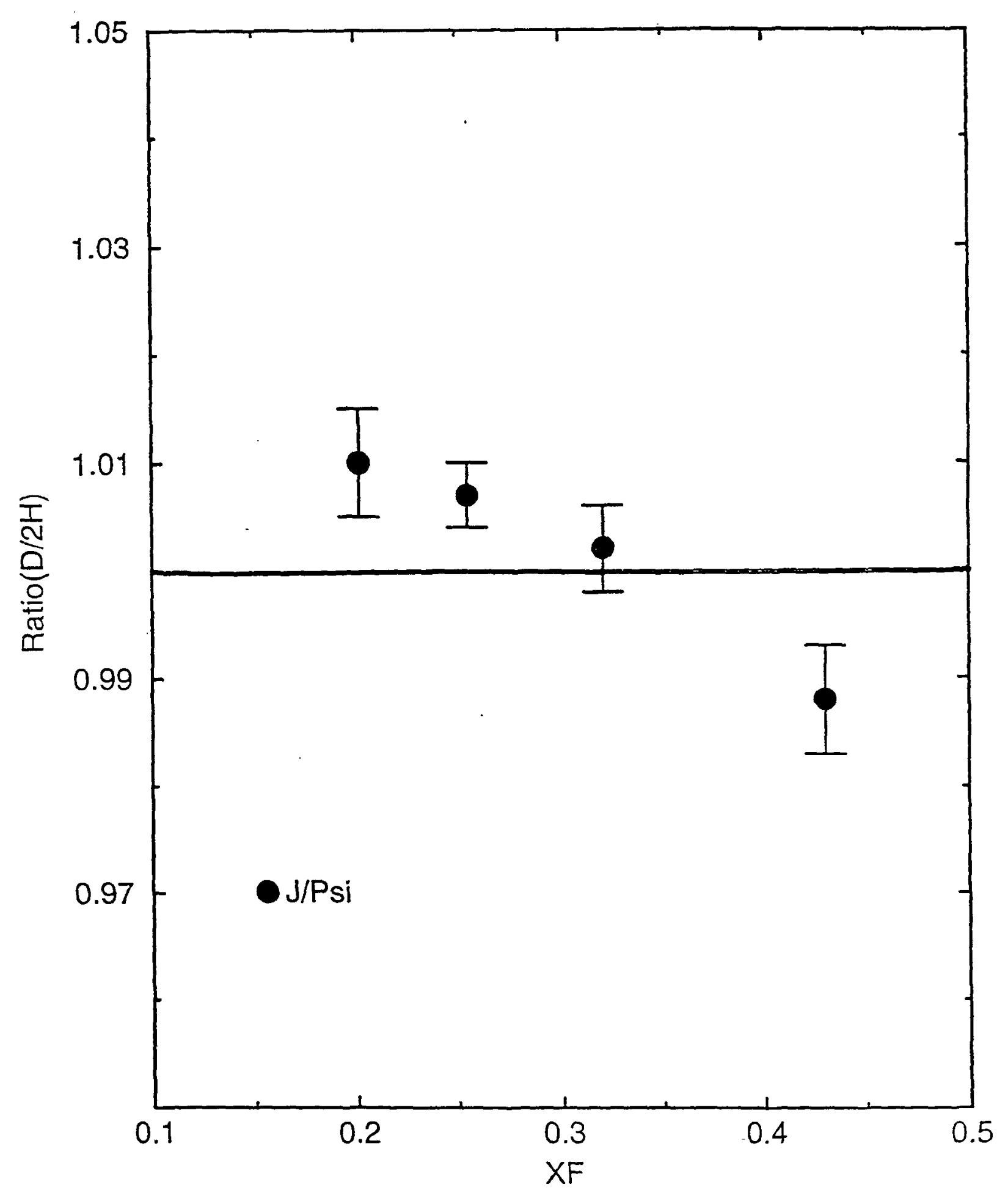




\section{Ratio of P-D to P-H Drell-Yan Yich As function of $\mathrm{x}_{2}$}

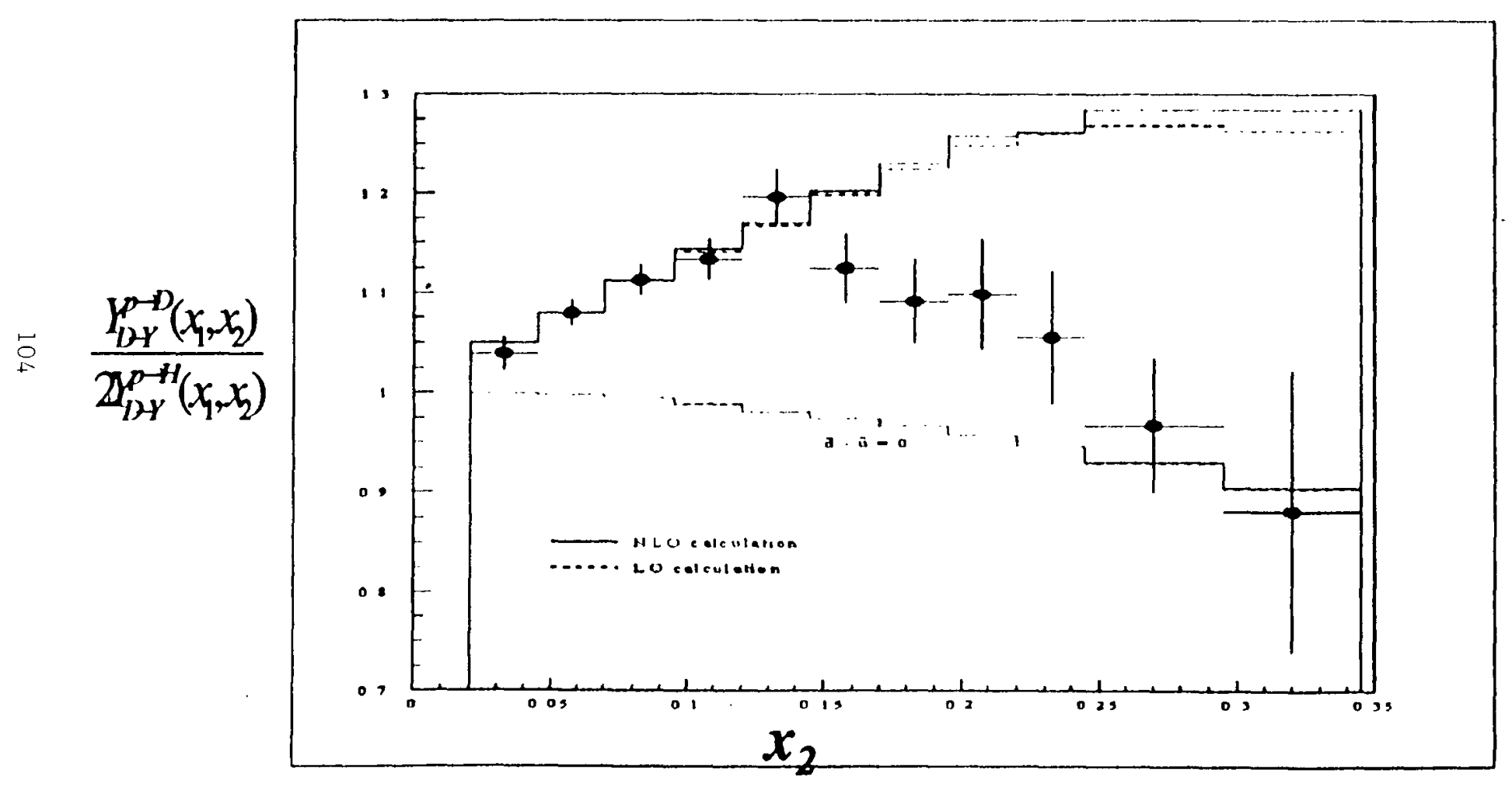




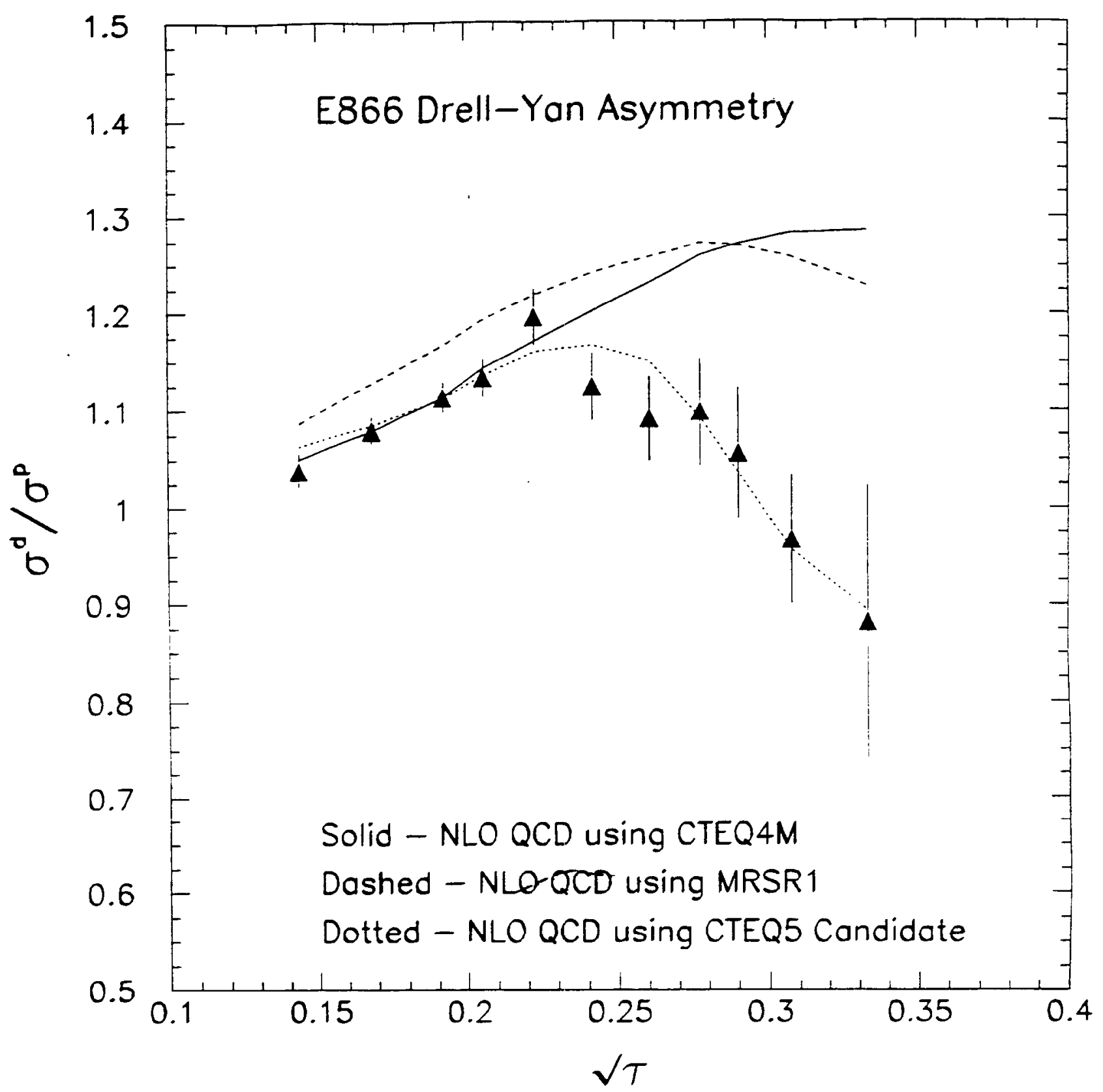




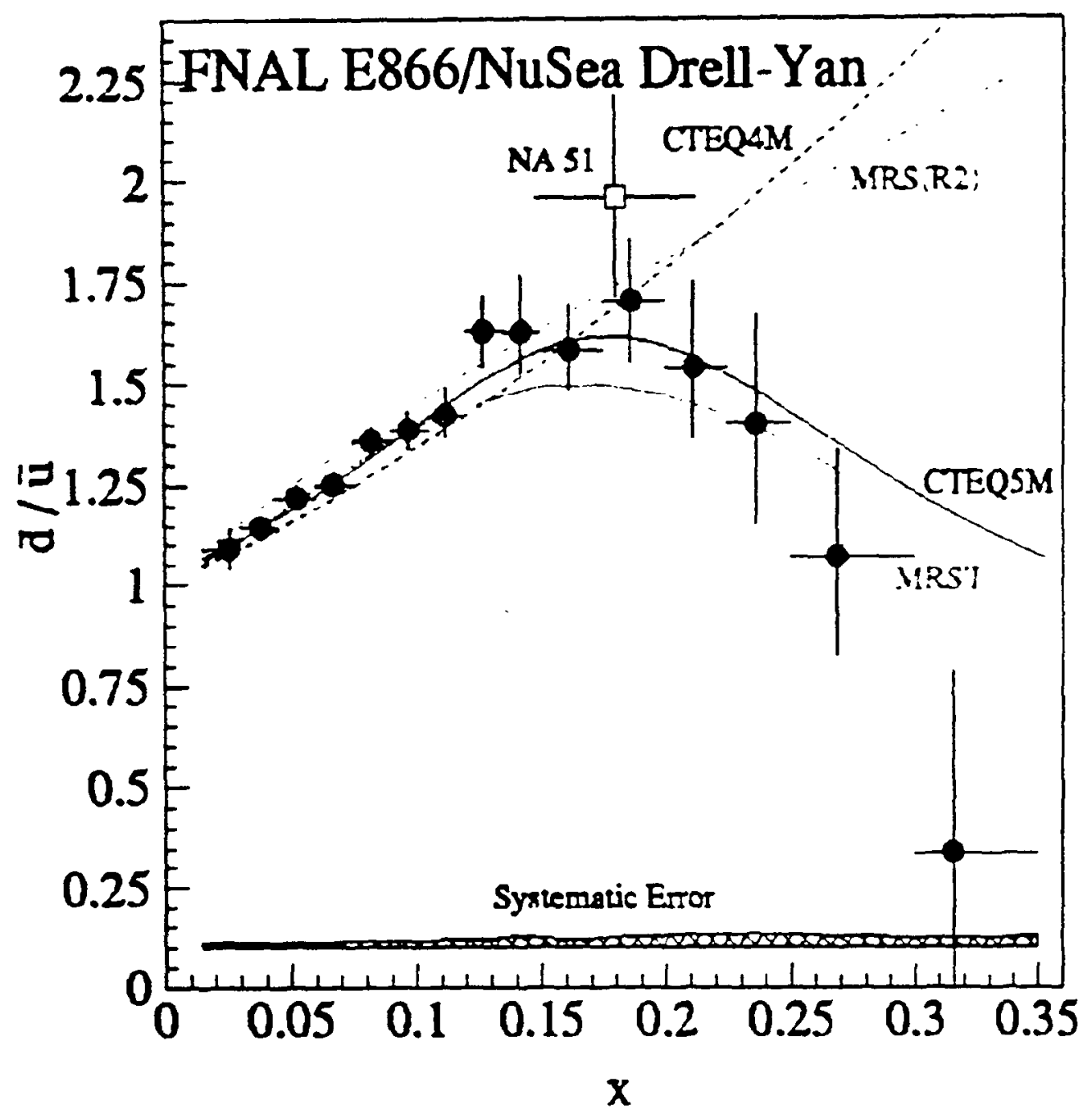




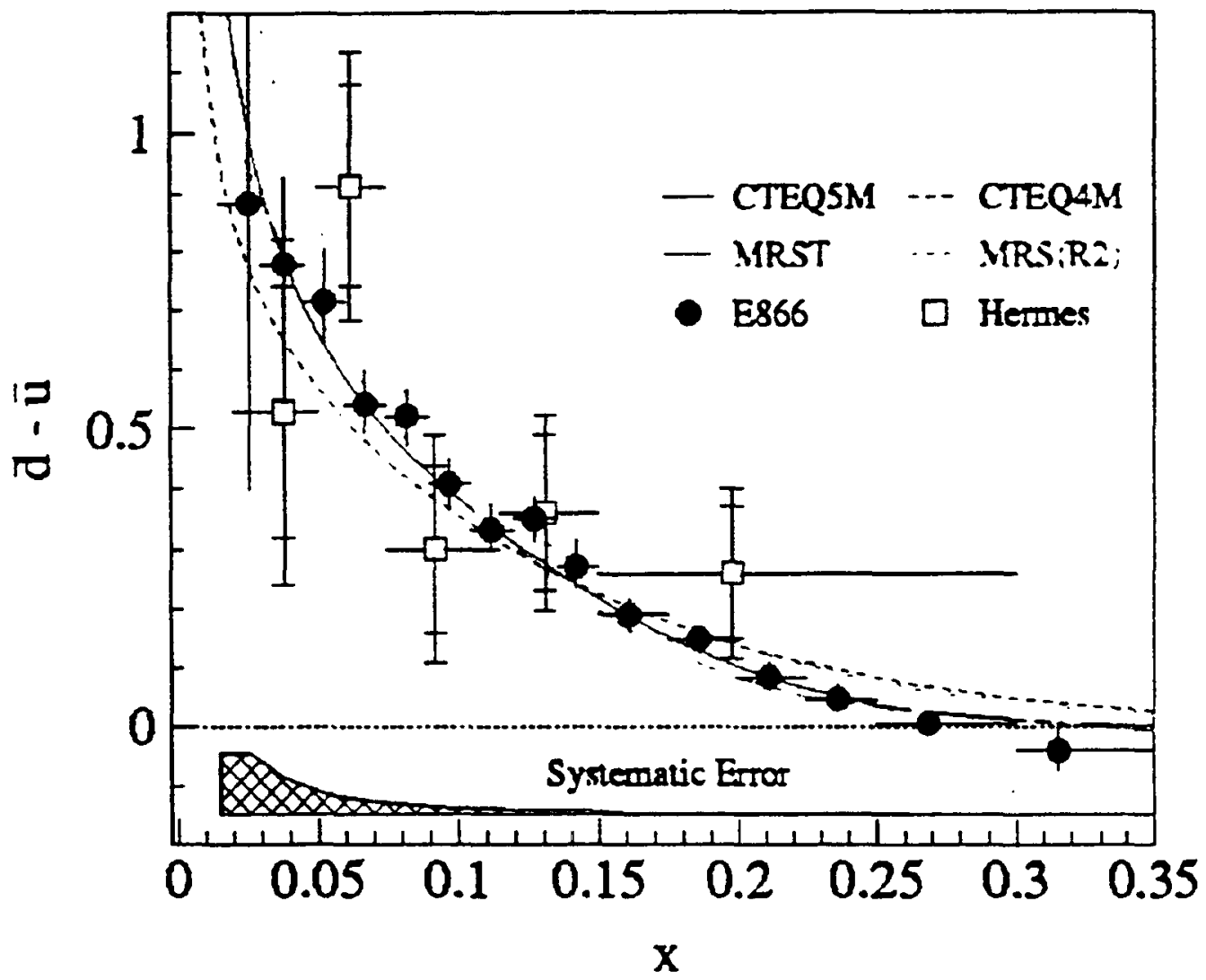

$$
\begin{aligned}
\int_{0.015}^{0.35}(\bar{d}-\bar{u}) d x & =0.0818 \pm 0.0082 \pm 0.0049 \\
\int_{0}^{1}(\bar{d}-\bar{u}) d x & =0.118 \pm 0.011
\end{aligned}
$$


$\overline{\boldsymbol{u}}_{p}, \overline{\boldsymbol{d}}_{p}$ Asymmetry Due to Pions

$p \rightarrow(1-a-b) p_{0}+a\left(\pi N_{0}\right)+b\left(\pi \Delta_{0}\right)$

$p \rightarrow(1-a-b) p_{0}+a\left(\frac{\pi^{0} p_{0}}{3}+\frac{2 \pi^{+} n_{0}}{3}\right)+b\left(\frac{\pi^{-} \Delta^{++}}{2}+\frac{\pi^{0} \Delta^{+}}{3}+\frac{\pi^{+} \Delta^{0}}{6}\right)$

$\boldsymbol{p}_{0}, \boldsymbol{n}_{0}, \Delta_{0}$ ect have flavor symmetric seas. $\pi^{+}=(\overline{\boldsymbol{d}} \boldsymbol{u}), \pi^{-}=(\overline{\boldsymbol{u}} \boldsymbol{d}), \pi^{0}=\frac{\boldsymbol{u} \overline{\boldsymbol{u}}+\boldsymbol{d} \overline{\boldsymbol{d}}}{2}$

$\bar{\delta}_{\infty} \quad \frac{\bar{d}_{p}(x)}{\bar{u}_{p}(x)}=\frac{\frac{5 a}{6}+\frac{b}{3}+S(x) / 2}{\frac{a}{6}+\frac{2 b}{3}+S(x) / 2}$

$\int_{0}^{1}\left[\bar{d}_{p}(x)-\bar{u}_{p}(x)\right] d x=\frac{1}{3}(2 a-b)$

In the chiral quark model

$$
\begin{aligned}
& u \rightarrow(1-\alpha) u+\alpha\left[\frac{\pi^{0} u}{3}+\frac{2 \pi^{+} d}{3}\right] \\
& u \rightarrow(1-\alpha) d+\alpha\left[\frac{\pi^{\prime \prime} d}{3}+\frac{2 \pi^{-} u}{3}\right] \\
& \frac{d_{p}(x)}{\bar{u}_{p}(x)}=\frac{\alpha\left[\begin{array}{c}
2 \cdot 5 \\
6
\end{array}+\frac{1}{6}\right]+S^{\prime}(x) / 2}{\alpha\left[\frac{5}{6}+\frac{2 \cdot 1}{6}\right]+S^{\prime}(x) / 2} \Rightarrow \frac{11}{7} \text { as } S^{\prime}(x) \Rightarrow 0
\end{aligned}
$$




\section{Pionic Content of the Nucleon}

$$
1 / 3(2 a-b)=0.118 \pm 0.011
$$

$$
a \cong 2 b
$$

So:

$a=0.24 \pm 0.022$ and $b=0.12 \pm 0.011$

Pionic Content:

$$
P_{p \pi}=a+b=0.36 \pm 0.028
$$




\section{Relative Fraction of the Sea From Pions}

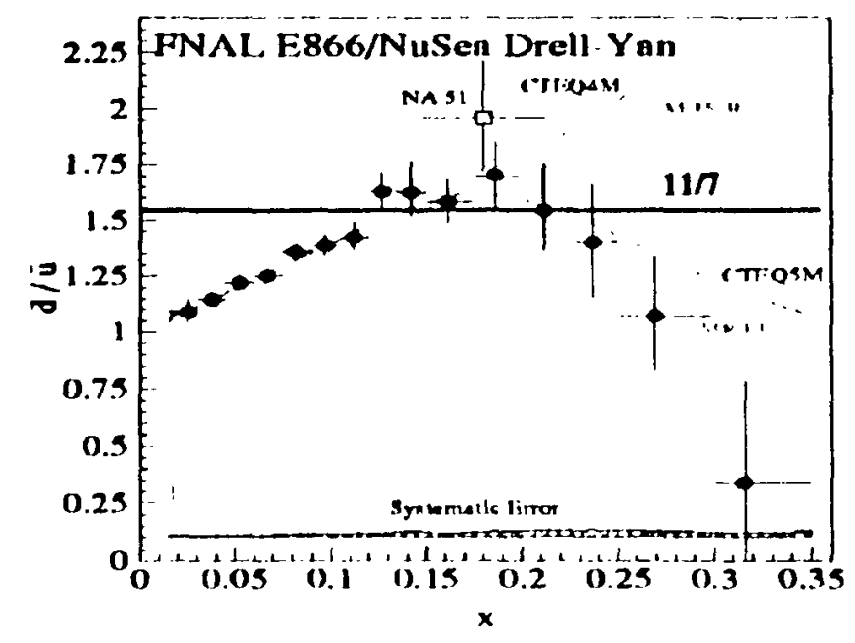

$$
\begin{aligned}
& \frac{\bar{d}_{p}(x)}{\bar{\pi}_{p}(x)}=\frac{S_{d}^{\pi}(x)+S_{0}(x) / 2}{S_{\pi}^{\pi}(x)+S_{n}(x) / 2}=\frac{5 a / 6+b / 3+5 / 2}{a / 6+2 b / 3+S_{0} / 2} \\
& \frac{\bar{d}_{p}(.15)}{\bar{\pi}_{p}(.15)}=\frac{1.44+3 S_{0}(.15)}{0.72+3 S_{0}(.15)}=1.65 \pm 0.05 \\
& \frac{S_{0}(.15)=0.13}{S_{\bar{d}}^{\pi}(.15)+S_{d}^{\pi}(.15)+S_{\bar{u}}^{\pi}(.15)}
\end{aligned}
$$




\section{Antiquarks in Nuclei}

Sophisticated models of nuclei all predict an increase in the number of pions per nucleon because pion exchange is the principle source of the N-N interaction.

For example: V.J. Pandharipande et al PRL. 51763 (83)

Change of Pion Momentum Distribution as Function of $k_{F}$

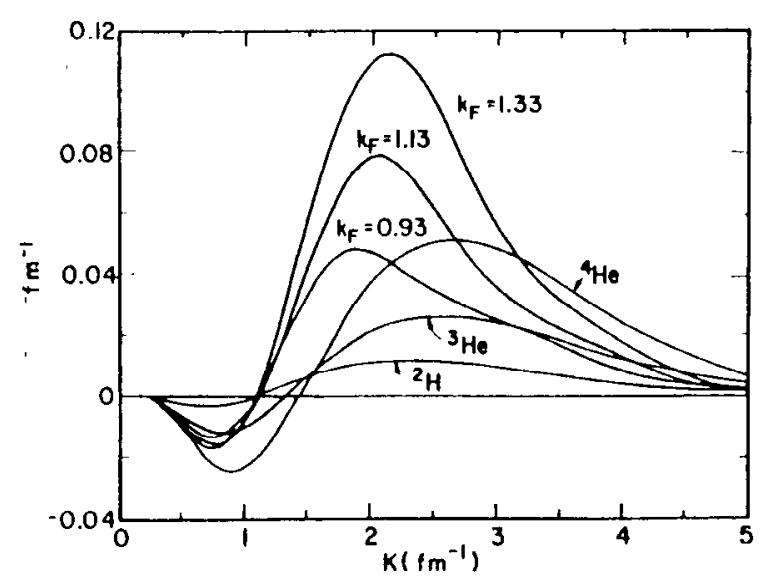

Pion Excess in Nuclei and Nuclear Matter

\begin{tabular}{ccl}
\hline & & \\
\hline & $\left(\delta n^{n}\right\rangle / A$ & $64 / A$ \\
$\mathrm{NM}, k_{\mathrm{F}}=0.93$ & 0.08 & 0.03 \\
$\mathrm{NM}, k_{\mathrm{F}}=1.13$ & 0.12 & 0.04 \\
$\mathrm{NM}, k_{\mathrm{F}}=1.33$ & 0.18 & 0.06 \\
${ }^{2} \mathrm{H}$ & 0.024 & 0.005 \\
${ }^{3} \mathrm{He}$ & 0.05 & 0.02 \\
${ }^{4} \mathrm{He}$ & 0.09 & 0.04 \\
${ }^{2{ }^{4} \mathrm{Al}}$ & 0.11 & 0.04 \\
${ }^{56} \mathrm{Fe}$ & 0.12 & 0.04 \\
${ }^{208} \mathrm{~Pb}$ & 0.14 & 0.05 \\
\hline
\end{tabular}




\section{Antiquarks in Nuclei (cont)}

No such increase in the antiquark distribution is observed

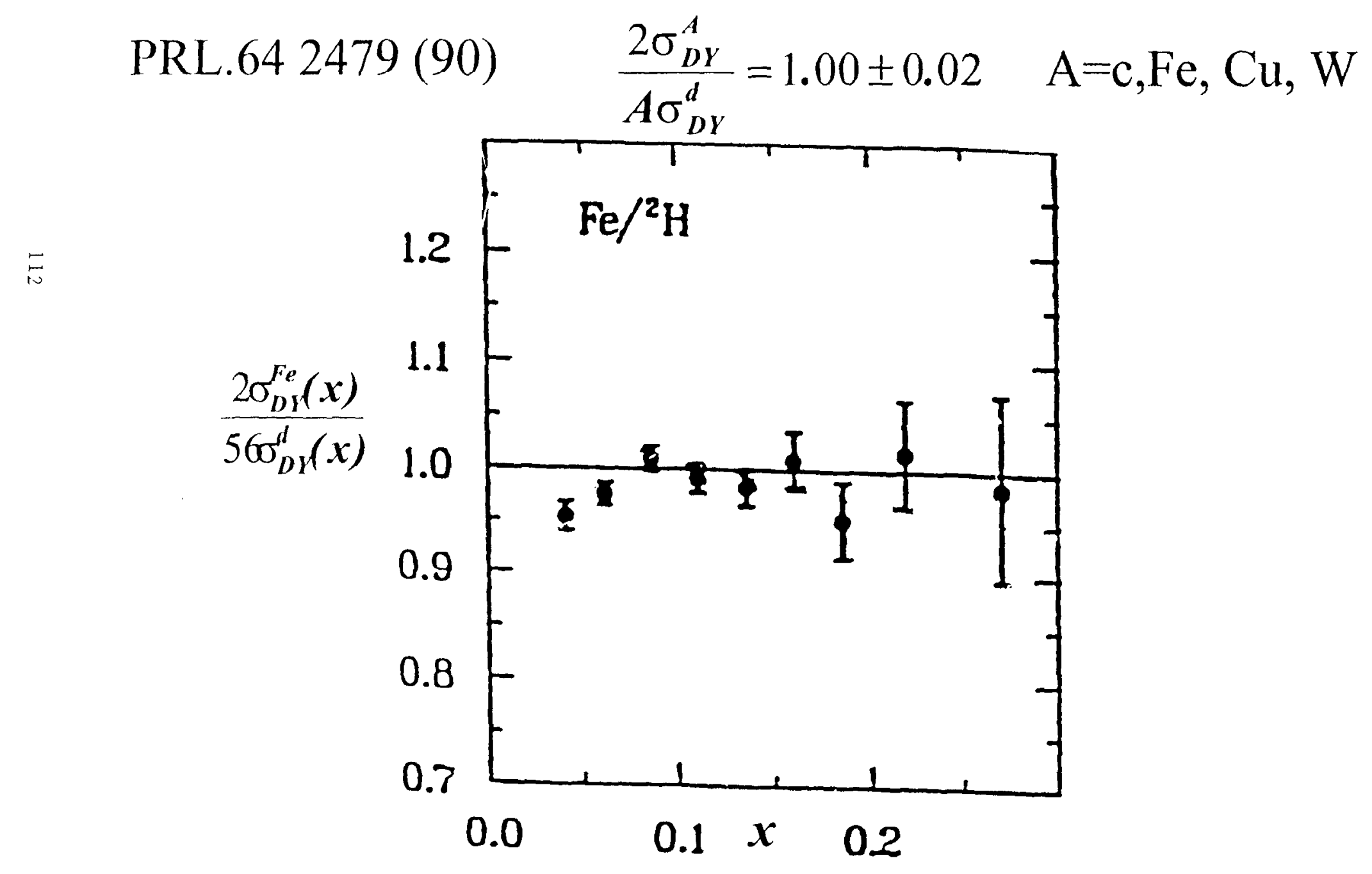




\section{Conclusions}

- E 866 confirms $\bar{d}_{p}(x) \geq \bar{u}_{p}(x)$ but with greater range and precision, allows the first determination of $\bar{d}_{p}(x)-\bar{u}_{p}(x)$

- $\bar{d}_{p}(x) / \bar{u}_{p}(x)$ for $x>0.2$ not yet explained in any model

- Conventional meson models explain $\bar{d}_{p}(x)-\bar{u}_{p}(x)$ but have lesser impact on $\Delta u_{p}-\Delta d_{p}$.

- Pions constitute a large fraction of the nucleon sea for $x>0.1$

- The expected increase in the number of pions/nucleon in nuclei is not observed ???? 


\title{
Physics at eRHIC - Selected Topics.
}

\author{
M W Krasny ${ }^{a}$ \\ aN2P3-CNRS, Universités Paris VI et VII, 4 pl Jussieu, T33-RdC, 75252 Paris, France \\ In high centre-of-mass energy lepton-nucleon collisions the space-time time resolution of \\ partonic interactions can be fine-tuned within a dynamical range which is unattainable in \\ hadronic collisions. Nuclei of variable atomic number allow to effectively tune the partonic \\ densities of hadronic media. The experimental program of high energy polarized (unpolar- \\ ized) electron-nucleon and electron-nucleus collisions (the eRIIC program) provides thus \\ an unique opportunity to investigate, with high precision, the microscopic picture of soft \\ and hard interactions of small and extended partonic systems. ${ }^{1}$.
}

\section{Introduction}

The eRHIC experimental program provides a large diversity of research domains ( see e.g the the series of lectures on eRHIC physics program [1]). In this short communication I choose to discuss only two aspects which, in my view, illustrate basic merits of such program with respect to other on-going and future experimental programs in high energy physics. Those are:

- the space-time resolution of electro-weak probes of hadronic matter

- a challenge to design and construct a unique detector providing full reconstruction of collisions of polarized and unpolarized electron, nulceon and nucleus beams.

\subsection{Space-time resolution}

The space-time charge resolution of the leptonic probe depends upon the following three kinematical variables: $x_{B j}$ - the invariant Bjorken variable, $Q^{2}$ - the invariant 4-momentum of the exchanged photon ( $W, Z$ boson) and $s$ - the centre-of-mass energy. These variables can be expressed in terms of the incoming and outgoing lepton 4-momenta $k$ and $k^{\prime}$ and by the 4-momentum of the nucleon $p$ :

$$
\begin{gathered}
Q^{2}=-q^{2}=-\left(k-k^{\prime}\right)^{2} \\
x_{B j}=Q^{2} /(2 p q) \\
s=(p+k)^{2}
\end{gathered}
$$

In the plane perpendicular to the collision axis the constituents of hadronic matter carrying electro-weak charges can be resolved within distances of $l_{t} \approx 1 / Q$. The corresponding space-time resolution in the longitudinal direction is eletermined by the value

'Talk at the BNL-eRIIIC' workshop, Brookhaven National Laboratory, July 2000 
of the invariant Bjorken variable $-x_{B j}$ and by the value of the reference frame-dependent Lorenz-factor of the nucleon (nucleus) $-\gamma: l_{l} \approx t \approx 1 /\left(2 \gamma M x_{B j}\right)$. In high energy collisions $l_{l}$ and $t$ are strongly correlated: $\left(t-l_{l}\right)^{2} \approx 1 / \mathrm{s}$ simplifying the large-distance structure of hadronic matter to "frozen configurations".

Strong interactions provide natural units for the resolution of transverse distances. Using $1 / \Lambda_{Q C D}$ and the inverse mass of the $\rho$ meson, $1 / m_{\rho}$ as "yardsticks" the following three kinematical regions can be defined:

- the photo-production region $\left(Q^{2}<\alpha_{1} \Lambda_{Q C D}^{2}\right)$, where the large-distance structure of hadronic matter is of importance and where the quasi-real photon interacts with the hadronic matter predominantly via the vector-meson component of its wave function.

- the transition region $\left(\alpha_{1} \Lambda_{Q C D}^{2}<Q^{2}<m_{\rho}^{2} / \alpha_{2}\right)$, where a direct coupling of the photon to a charged partons become important

- the deep inelastic region $\left(Q^{2}>m_{\rho}^{2} / \alpha_{2}\right)$, where the direct coupling of the photon to a charged parton dominate

These kinematical regions have rather fuzzy boundaries corresponding to $\alpha_{1}$ and $\alpha_{2}$ values in the range of approximately 0.1-1.0.

The natural scales of the resolution of the longitudinal distances in electron-nucleon and electron-nucleus collisions are: the size of nucleons $R_{N}$ and size of nuclei $R_{A}$ defined here in a Lorenz-invariant way as distances over which the valence quarlss of the nucleon (nucleus) are localized. These values define three kinematical regions:

- large $x_{B j}$ region $\left(x_{B_{j}}>1 /\left(2 M R_{N}\right) \approx 0.1\right)$ where the the photon (W, $Z$ boson) interaction with the nucleon (nucleus) is localized within the longitudinal distances smaller than the nucleon size

- intermediate $x_{B j}$ region $\left(1 /\left(2 M R_{A}\right) \approx 0.01<x_{B}<1 /\left(2 M R_{N}\right) \approx 0.1\right)$ where the photon interacts coherently within the longitudinal distances exceeding the size of the nucleon

- small $x_{B j}$ region $\left(x_{B j}<1 /\left(2 M R_{A}\right) \approx 0.01\right)$ where the light-cone-coherent interaction of the photon extends over the longitudinal distances exceeding the size of the nucleus

The $\left(x, Q^{2}\right)$ region which can be covered in electron-proton (electron-nucleus) collisions at eRHIC and that for TJNAF program are shown in Fig. 1.

The main distinction between the TJNAF and eRHIC kinematical domains, resulting from different centre-of-mass energies, is that in the latter case a broad range of $x_{B} ;$ values, in particular the small $x_{B}$, region, can be studied in the DIS regime where the relevant hadronic degrees of motion are quarks and gluons.

The small $x_{B j}$, large coherence length, DIS processes can be viewed in two equivalent and complementary ways. In the Bjorken reference frame in which the nucleon (nucleus) moves with asynutotically large nomentum the photon collides with dr-localized pertons. 


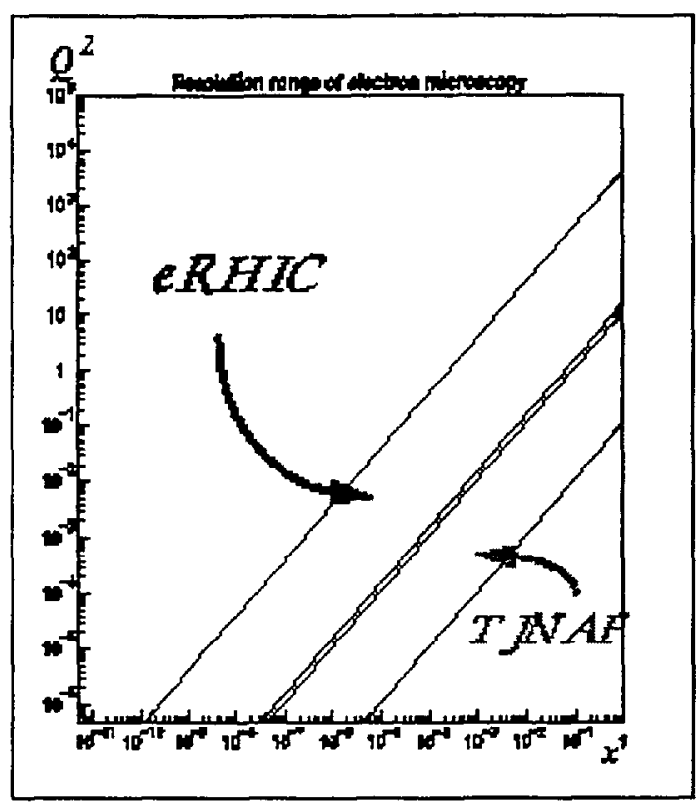

Figure 1. The kinematical region covered by the eRHIC and TJNAF experimental programs.

which are described by the light-cone wave function of the nucleon (nucleus). In this picture nuclei can be considered as sources of variable ( $A^{1 / 3}$-dependent) quark and gluon densities.

In the rest frame of the nucleon (nucleus) the small $x_{B j}$ DIS collisions can be viewed as coherent scattering of various quark-gluon Fock components of the virtual photon wave function. In this picture nuclei can be considered as effective ( $A^{1 / 3}$-dependent) filters of various Fock projections of the virtual photon wave function. The dynamics of quark and gluon interactions is expressed in this frame in terms of the cross sections for interactions of $q \bar{q}, q \bar{q} g$ and other Fock states of the virtual photon with the nucleon (nucleus).

\subsection{Detector - the first design trial}

The dedicated detector for the eRHIC experimental program has to combine two basic functions. It has to measure both hard partonic processes and the soft remnants of the electrons, nucleons and nuclei. The first design attempt for such a detector has been presented and discussed both at the Yale and BNL workshops [2]. The following criteria were self-imposed for this design:

- The detector had to be common for ep, eA, pp and pA collisions to study both the hard scattering of partons and the beam particle dependent subtle mcdium effects.

- It should allow for the reconstruction of "complete" ep and eA events (covering the proton fragmentation region in $\mathrm{pp}$ and $\mathrm{pA}$ interaction remains, as much as the muon dotection system, optional). 
- The beam crossing optics had to be designed trying to minimize the clash with the existing interaction region optics.

- The existing RHIC magnets had to be used, as much as they can be useful, in the spectrometer design.

- The electron insertion should provide a functionality of the spin rotator.

- The design should remain invariant of the choice of Ring-Ring vs. Linac-Ring eA(p) collisions.

- The emphasis had to put on the detector quality in the fragmentation rather than in the central region.

- The detector should provide precise luminosity monitoring and good experimental control of radiative corrections for electron induced reactions.

The general layout of the detector geometry is shown in Fig. 2.

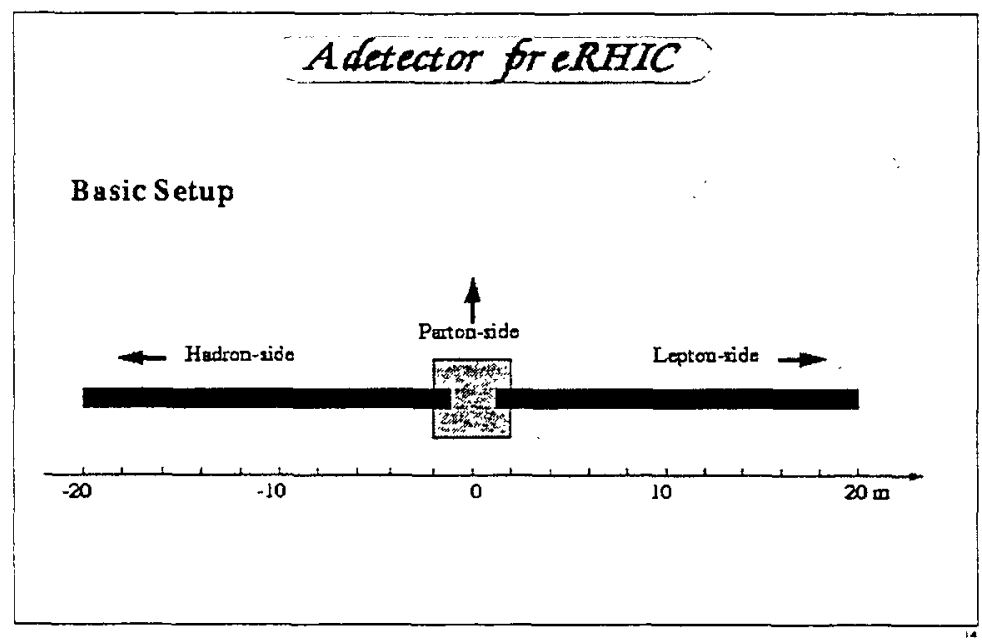

Figure 2. The detector geometry.

The function of the central detector, shown in Fig. 3, is to determine the kinematics of hard processes by measuring the momenta and angles of outgoing leptons quark and gluon jets.

The proton (nucleus) fragmentation region is covered by the high rigidity and medium rigidity spectrometers shown in Fig. 4. The function of these spectrometers is to measure wounded and evaporated nuclcons, nuclear fragments as well as other low-angle particles. The spectrometers involve the tracking modules and calorimeter stations and use, in mcastring the momentum of clarged particles, the magnetic field of the DO and DE hlagnets 


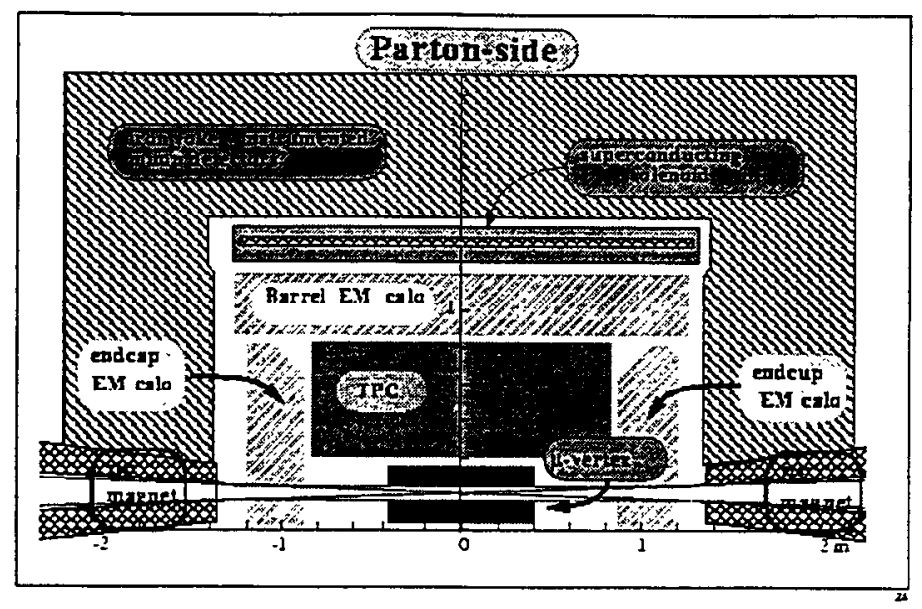

Figure 3. The central detector.

The detection system in the electron fragmentation region, shown in Fig 5, defines the minimal setup for the electron-nucleon (electron-nucleus) collisions. It provides a precise tagging of DIS and photoproduction processes and measures the low angle photons for luminosity measurement and experimental means to control electromagnetic radiative corrections.

The 2.3 Tm bending power of the DE magnet distributed over 3 meters allows to insert electrons to the $\mathrm{pP}, \mathrm{pA}, \mathrm{AA}$ collision point under the constraint of not changing the DX and D0 magnet configurations, while keeping the synchrotron radiation power at tolerable level (note that the electron currents 10 time higher than those at DESY can be tolerated at eRHIC if maximal energy of the electron beam stays below $10 \mathrm{GeV}$ ). The deflection angle of $70 \mathrm{mrad}$ allows for rotation of the electron spin by $90 \mathrm{deg}$. The main advantage of such a collision optics is to avoid by-passing of one of the proton (ion) ring in the detector zone and to assure "no-clash" operation of eRHIC and RHIC machines.

\section{Selected examples of research domains for eRHIC - example of eA collisions}

High-energy electron-nucleus collisions were discussed at several workshops [3], [4], [5], $[6]$. The research domains, which in my view are both exciting and unique for the eRHIC program include:

- Study of large density partonic systems. Search for new non-linear QCD phenomena.

- Study of colour singlet (diffractive) processes. Understanding Pomeron and rapidity gaps.

- Study of the space time structure of strong interactions. Understanding of confinement mechanism. 


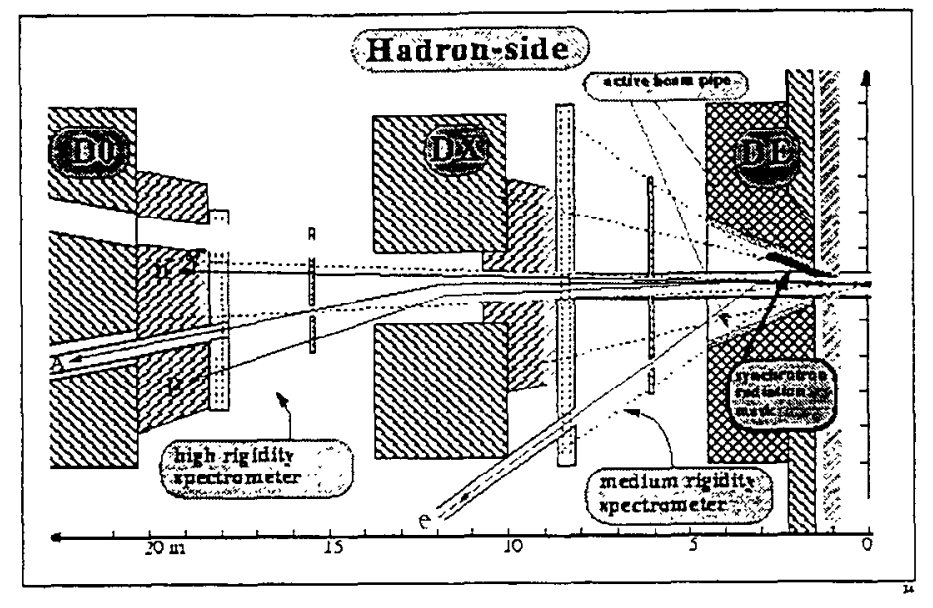

Figure 4. Instrumentation of the proton (nucleus) fragmentation region.

- Study of exclusive and semi-exclusive processes. Rigidity of nucleons and nuclei (skewed partonic distributions)

- Study of the real and virtual photon interactions. Understanding the transition between hard (QCD-perturbative) and soft (QCD-non-perturbative) processes

- Study of luminous photon-photon scattering

- Study of nucleus evaporation process initiated by hard point-like interaction (fragmentation of nucleus which "lost one of its partons")

- Exclusive studies of disintegration processes of light nuclei in photon-nucleus interactions study of the electron-neutron scattering with proton spectator tag

- etc.

The shopping list of "bread and butter" precision measurements which can be made at eRHIC include:

- $F_{2}^{A}$ and $F_{L}^{A}$ structure functions: inclusive and tagged by the number of wounded nucleons and evaporation fragments

- A-dependence of the gluon distribution

- Jet spectra and single particle inclusive spectra in the photo-production, DIS, and the transition regions

- A-dependence of the vector meson production

- A-dependence of the open charm and beauty production 


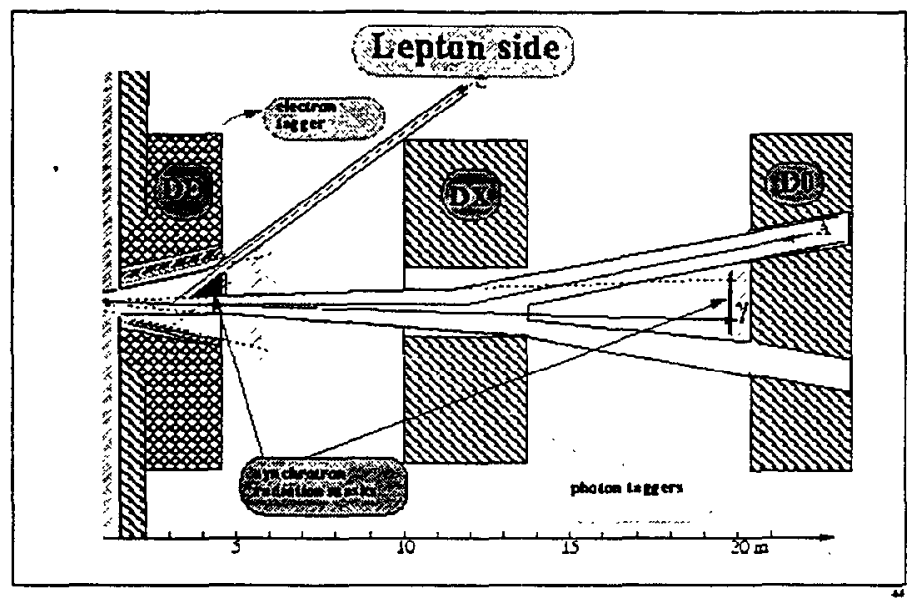

Figure 5. The minimal set-up for the instrumentation covering the electron fragmentation region.

- A-dependence of the fraction of rapidity gap events

- A-dependence of jet profiles and jet energy loss

- Fragmentation spectra of tagged low-x partons in nuclear medium, also in correlation with the observed number of wounded nucleons and evaporation fragments

- Particle multiplicities and particle correlations in $\gamma^{*} A$ and $\gamma A$ scattering

- Bose-Einstein correlations and their A-dependence

The above measurements can be derived from the on-going and future programs at, DESY and CERN. They can complement, in my view more important, curiosity driven generic research program of QCD in its full complexity (beyond the processes controlled by its perturbative approach) and involving studies of QCD vacuum and partonic structure of large distance strong interactions.

\section{REFERENCES}

1. M.W. Krasny, Four lectures on the physics of the eRHIC project and on the detector design. See http://quark.phy.bnl.gov/ raju/eRHIC.html.

2. M.W. Krasny, Talk at the Yale workshop and the discussion on detector issues, Yale, April 2000.

3. The nuclear session of the Paris 1995 workshop is summarized in the Proceedings of the DIS-1995 workshop, Paris 1995.

4. Studies on various aspects of experimental program with light and heavy nuclei in HERA are summarized in the Proceedings of the Future Physics at HERA Workshop. DESI. Hamburg - 1996. 
5. The comparison of The HERA, GSI-ENC and ELFE@HERA experimental programs can be found in the Proceedings of the SEEHEIM workshop Seeheim 1997.

6. The eRHIC physics program was discussed during the Yale workshop Yale, April 2000. 


\title{
Exploring skewed parton distributions with polarized targets *
}

\author{
M. Diehl \\ Stanford Linear Accelerator Center, Stanford Universily, \\ Stanford, CA 94309
}

\section{The physics of skewed parton distributions}

In recent years much progress has been made in the theory of skewed parton distributions (SPDs). Unifying the concepts of parton distributions and of hadronic form factors, they contain a wealth of information about how quarks and gluons make up hadrons. Advances in experimental technology raise hope to study the exclusive processes where these functions appear.

While the usual parton distributions are matrix elements of quark or gluon operators for a given hadron state $p$, SPDs are obtained from the same operators sandwiched between two hadron states $p$ and $p^{\prime}$ with different momenta, corresponding to the finite momentum transfer the hadron undergoes in an exclusive process. A good example for this is deeply virtual Compton scattering (DVCS). This is the process $\gamma^{*} p \rightarrow \gamma p$ (measured in electroproduction $e p \rightarrow e p \gamma$ ) in the kinematical regime where the photon virtuality $Q^{2}=-q^{2}$ and the energy squared $W^{2}=(p+q)^{2}$ are large, while the invariant momentum transfer $t=\left(p-p^{\prime}\right)^{2}$ to the proton is small. If $Q^{2}$ is large enough, the transition amplitude factorizes [1] into a perturbatively calculable subprocess at the level of quarks and gluons and an SPD, which encodes the nonperturbative dynamics relating the quarks or glvons with the proton states (Fig. 1a).

The key difference between the usual parton distributions and their skewed counterparts can be seen by representing them in terms of the quark and gluon wave functions of the hadron. The usual parton distributions are obtained from the squared wave functions for all partonic configurations containing a parton with specified polarization and longitudinal momentum fraction $x$ in the fast moving hadron (Fig. 2a). This represents the probability for finding such a parton. In contrast, SPDs represent the interference of different wave functions, one where a parton has momentum fraction $x+\xi$ and one where this fraction is $x-\xi$ (Fig. 2b). SPDs thus correlate different parton configurations in the hadron at the quantum mechanical level. There is also a kinematical regime

\footnotetext{
-Work supported by Department of Energy contract DE-AC03-76SF00515 and by the Feodor Lynen Program of the Alexander von Humboldt Foundation.
} 


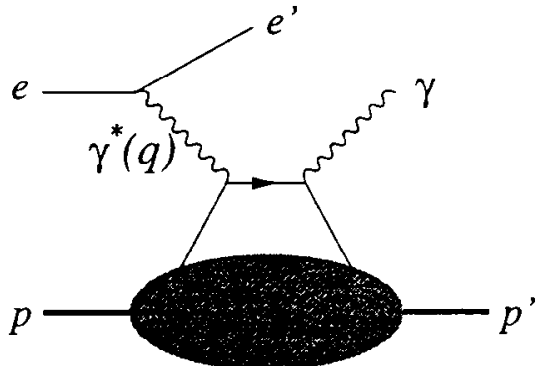

(a)

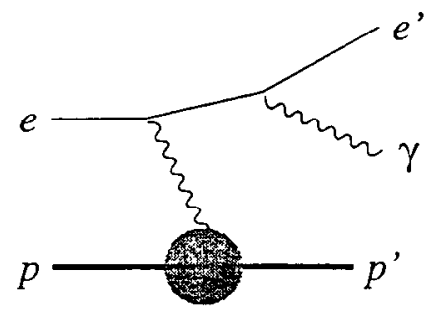

(b)

Figure 1: (a) A Born level diagram for DVCS. The blob represents a skewed quark distribution. (b) A diagram for the Bethe-Heitler process. The blob here stands for an elastic proton form factor.

where the initial hadron emits a quark-antiquark or gluon pair (Fig. 2c). This has no counterpart in the usual parton distributions and carries information about $q \bar{q}$ and $g g$-components in the hadron wave function.

Apart from the momentum fraction variables $x$ and $\xi$ SPDs depend on the invariant momentum transfer $t$. This is an independent variable because the momenta $p$ and $p^{\prime}$ may differ not only in their longitudinal but also in their transverse components. SPDs thus interrelate the longitudinal and transverse momentum structure of partons within a fast moving hadron.

SPDs have a rich structure in the polarization of both the hadrons and the partons. For quarks four different combinations contribute to DVCS. The functions $H_{q}$ and $E_{q}$ are summed over the quark helicity, and $\tilde{H}_{q}$ and $\tilde{E}_{q}$ involve the difference between right and left handed quarks. $H_{q}$ and $\tilde{H}_{q}$ conserve the helicity of the proton, whereas $E_{q}$ and $\tilde{E}_{q}$ allow for the possibility that the proton helicity is flipped. In that case the overall helicity is not conserved: the proton changes helicity but the quarks do not, so that angular momentum conservation has to be ensured by a transfer of orbital angular momentum (Fig. 3a). This is only possible for nonzero transverse momentum transfer, and therefore cannot be observed with ordinary parton distributions, where the momenta $p$ and $p^{\prime}$ are equal. That SPDs deeply involve the orbital angular momentum of the partons is epitomized in Ji's sum rule [2], which states that the second moment $\int d x x\left[H_{q}(x, \xi, t)+E_{q}(x, \xi, t)\right]$ is a form factor whose value at $t=0$ gives the total angular momentum carried by quarks, both its spin and orbital part. For gluons there are corresponding distributions $H_{g}, E_{g}, \bar{H}_{g}, \bar{E}_{g}$, and an analogous sum rule exists.

There are SPDs with yet another spin structure [3]. Distributions llipping the quark helicity are the skewed counterparts of the usual quark transversity distribution, but no process is known at present where they contribute [4]. In the gluon sector there are distributions which change the gluon helicity by two 


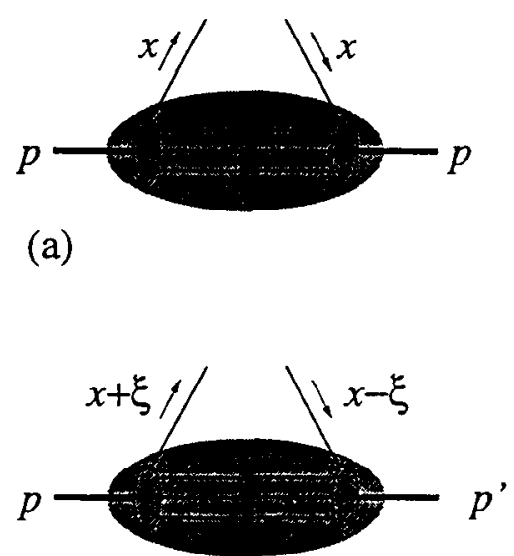

(b)
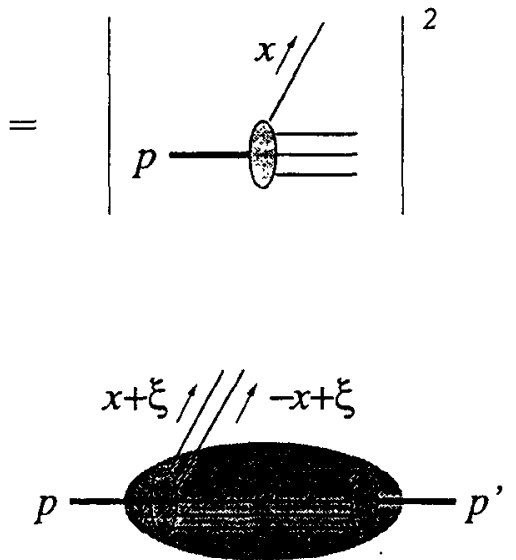

(c)

Figure 2: (a) Usual parton distribution, representing the probability to find a parton with momentum fraction $x$ in the nucleon. All configurations of the spectator partons are summed over. (b) SPD in the region where it represents the emission of a parton with momentum fraction $x+\xi$ and its reabsorption with momentum fraction $x-\xi$. (c) SPD in the region where it represents the emission of a parton pair. Here $x+\xi>0$ and $x-\xi<0$.

units. Because of angular momentum conservation such a double helicity flip can be realized with ordinary parton distributions only for targets of spin 1 or higher, whereas the skewed distributions are accessible for nucleons if there is a finite transverse momentum transfer (Fig. 3b). These distributions appear in DVCS at the $\alpha_{s}$ level $[3,5,6]$.

\section{Phenomenology: the potential of polarization}

The principal reactions where SPDs can be accessed are DVCS and exclusive meson electroproduction, $e p \rightarrow e p M$, where the meson $M$ replaces the real photon in the final state of Compton scattering. DVCS is special in its phenomenology, because it interferes with the Bethe-Heitler process, where the real photon is radiated from the lepton (Fig. 1b). In kinematics where the BetheHeitler contribution is large compared with the Compton process, one can use their interference term to study the latter, because the former can be calculated given knowledge of the Dirac and Pauli form factors $F_{1}(t)$ and $F_{2}(t)$ of the proton. This offers the unique possibility to study Compton scattering at the amplitude level, including its phase. The even larger pure Bethe-Heitler contribution can be removed from the cross section by various asymmetries. Different information on the interference term is obtained by reversing the lepton beam 


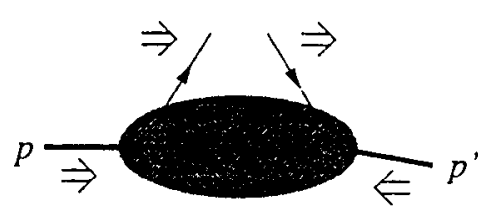

(a)

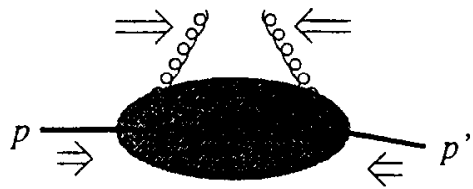

(b)

Figure 3: (a) SPD which flips the helicity of the proton but not of the quark. A unit of orbital angular momentum must be transferred. (b) SPD which flips the gluon helicity. Again there must be transfer of orbital angular momentum.

charge and by various asymmetries of the lepton and the proton polarizations.

To fully explore the physics of SPDs one will want to disentangle the contributions from the various spin and flavor combinations. For flavor the combined information from DVCS and from the production of mesons with different quantum numbers will be necessary. As for the spin degrees of freedom, the functions $H$ and $E$ appear for vector mesons, $\tilde{H}$ and $\tilde{E}$ for pseudoscalar mesons, and all of them for DVCS. To make further progress (and for instance to obtain the combination $H+E$ occurring in Ji's sum rule) it is mandatory to perform measurements with polarized protons. While it is true that one can access polarization dependent SPDs in unpolarized collisions, one needs polarization in order to disentangle the different distributions.

With some exceptions, the unpolarized cross section and the different polarization asymmetries in DVCS involve all four distributions $H, E, \tilde{H}, \tilde{E}$ [7]. Typically, however, some of them are suppressed by kinematical prefactors. The unpolarized DVCS cross section is dominated by $H \cdot H+\tilde{H} \cdot \tilde{H}$, whereas with longitudinal target polarization one is mostly sensitive to $I I \cdot \tilde{H}$. This provides a handle to separate $H$ and $\tilde{H}$, with smaller contributions from $E$ and $\tilde{E}$. The same is possible with the interference between DVCS and Bethe-Heitler, where with an unpolarized target one mainly looks at $F_{1} \cdot H+\left(F_{1}+F_{2}\right) \cdot \xi \tilde{H}$, and with longitudinal target polarization mainly at $F_{1} \cdot \tilde{H}+\left(F_{1}+F_{2}\right) \cdot \xi H$. A way to access $E$ and $\tilde{E}$ without a large contribution from $H$ and $\tilde{H}$ is the transverse target polarization asymmetry in the DVCS cross section, which is a sum of terms where $E$ or $\tilde{E}$ are multiplied with $H$ or $\tilde{H}$. 'The same type of separation can be made in exclusive meson production [8].

The gluon helicity flip distributions discussed above can be isolated in the DVCS cross section through the angular distribution of the final state [5]. This can be done without target polarization, but target polarization enhances the possibilities of extraction. With a longitudinally polarized target one generates a $\sin 3 \varphi$ dependence in the interference between the Compton and Bethe-Heitler processes that is otherwise absent [6], and target polarization is again required for separating the different helicity flip SPDs. 


\section{$3 \quad$ Small $x$ or not small $x$}

The momentum fraction variables $x$ and $\xi$ of the skewed distributions play different roles in the amplitude of physical processes: $x$ parameterizes a loop momentum and is always integrated over, whereas $\xi$ is fixed to $x_{B} /\left(2-x_{B}\right)$ by external kinematics, where $x_{B}=Q^{2} /(2 p \cdot q)$ is the Bjorken variable as defined for deep inelastic scattering. Broadly speaking, the loop integrals will however probe smaller values of $x$ when $\xi$ becomes small.

The physics questions one aims to study with SPDs typically change with the value of $\xi$. At moderate or large $\xi$ one expects to be most sensitive to the effect of the skewed kinematics, and to learn about the interference between different wave functions, including the regime $-\xi<x<\xi$ where one probes quark-antiquark and gluon pairs in the target wave function.

As $\xi$ becomes very small, the relative difference of momentum fractions in the SPD is small over an increasingly important region of $x$. The hope here is that the measurement of SPDs can help constrain the usual parton distributions. Most studies have so far focused on vector meson production at small $x_{B}$, which is dominated by the square of the skewed gluon distribution $H_{g}$. Data from the HERA collider have already been used in an attempt to get information on the gluon density $g(x)$ at small $x$ [9]. It is not a trivial task to relate a function $H_{g}(x, \xi, t)$ of three variables to $g(x)$, but theoretical arguments [10] building on the QCD evolution equations for SPDs suggest that at small enough $x_{B}$ this can be done within reasonable uncertainties. There have been efforts to find a similar way to constrain the polarized gluon density $\Delta g(x)$ from $\tilde{H}_{g}(x, \xi, t)$ [11], but it turns out that for vector meson production this is not possible at the leading-twist level [12]. Beyond leading twist theory is plagued with large contributions from infrared regions if the meson is made from light quarks [13]. The only known process where $\bar{H}_{g}$ comes in is DVCS, where it appears at the level of $\alpha_{s}$ corrections (as $\Delta g(x)$ does in polarized deep inelastic scattering).. No studies have yet been made of whether this might help in pinning down $\Delta g(x)$.

Where the transition is between "large" values of $\xi$, where one hopes to learn from the effect of the longitudinal momentum asymmetry, and "small" values, where one expects this effect to be sufficiently under control to provide handles on the usual parton densities, is not known. This will probably have to be explored in the data. Many studies will not need to go to very small $\xi$ (note for example that Ji's sum rule involves $x\left(H_{q}+E_{q}\right)$ where small $x$ is suppressed), and others will aim to get $\xi$ as small as possible. As far as spin is concerned, one expects that the parton helicity independent distributions will become more and more dominant at small $x$, just as happens with ordinary distributions.

Whereas moderate or large values of $x_{B}$ are kinematically accessible for a wide range of collision energies (although with different counting rates that need to be studied), small $x_{B}$ is of course the realm of high-energy machines. A specific feature of DVCS is that at given ep collision energy and $Q^{2}$ the interference term with Bethe-Heitler favors the smallest available $x_{B}$, whereas the DVCS cross section reaches out to higher values, in a similar way as inclusive deep inelastic scattering. Given the rather complex structure of the interference 
term and the various combinations of polarization, it is difficult without detailed numerical studies to determine the "optimal" machine energy for studies of SPDs, even in a given range of $\xi$.

\section{Experimental challenges (a theorist's view)}

The experimental study of SPDs faces several tasks:

1. Luminosity: some of the interesting channels have relatively small cross sections. This includes DVCS, whose cross section goes like $\alpha_{\mathrm{em}}^{3}$. For a quantitative study of SPDs, event statistics must be sufficient to allow binning in the different variables, $Q^{2}, x_{B}, t$, and to study angular correlations.

2. Large $Q^{2}$ : in order to be in the regime where the QCD factorization theorems hold, one needs sufficiently large $Q^{2}$. What "sufficient" is has to be determined experimentally for each channel, by testing the predicted power-law behavior in $Q^{2}$ and the predicted pattern of angular distributions. This requires lever arm in $Q^{2}$, and to be on safe ground one will want to achieve large $Q^{2}$. For given $x_{B}$ it imposes both kinematical constraints on the machine (not very serious at high energies) and requires again good luminosity, because of the expected decrease of cross sections as a power-law in $1 / Q$.

3. Exclusivity: For quantitative studies it is paramount that one knows the final state of the reaction. The processes $\gamma^{*} p \rightarrow \gamma p$ and $\gamma^{*} p \rightarrow M p$ compete with the cases where the proton dissociates into a low-mass system, say a $\Delta$ or the $N \pi$ continuum. Interesting in themselves, these reactions involve SPDs for the transition from the target proton to the hadronic system in question. In order to extract specific SPDs it is of course necessary to separate the corresponding channel. This is especially crucial for spin studies, since the spin structure of the transitions $p \rightarrow \Delta$ and $p \rightarrow p$ is different. In the case of DVCS one also finds that with proton dissociation polarization asymmetries no longer remove the Bethe-Heitler contribution to the cross section (only the lepton charge asymmetry still does).

Detection and identification of the scattered proton (or hadronic system) is therefore necessary, unless the resolution in energy and momentum is sufficient to use the missing-mass technique with an accuracy of the order of the pion mass.

In addition to these requirements there is the strong physical motivation to have a polarized lepton beam and a polarized proton target. Given the boundary conditions 1. and 3 . just discussed, it is not clear at present whether this can be achieved with fixed targets, and a polarized collider may be the most promising option. The wealth of physics to be learned about by studying skewed parton distribution goes with formidable challenges for experiment. 


\section{References}

[1] A. V. Radyushkin, Phys. Rev. D56, 5524 (1997) [hep-ph/9704207];

J. C. Collins and A. Freund, Phys. Rev. D59 (1999) 074009 [hep$\mathrm{ph} / 9801262]$.

[2] X. Ji, Phys. Rev. Lett. 78 (1997) 610 [hep-ph/9603249].

[3] P. Hoodbhoy and X. Ji, Phys. Rev. D58, 054006 (1998) [hep-ph/9801369].

[4] M. Diehl, T. Gousset and B. Pire, Phys. Rev. D59, 034023 (1999) [hep$\mathrm{ph} / 9808479]$;

J. C. Collins and M. Diehl, Phys. Rev. D61, 114015 (2000) [hep$\mathrm{ph} / 9907498]$.

[5] M. Diehl, T. Gousset, B. Pire and J. P. Ralston, Phys. Lett. B411, 193 (1997) [hep-ph/9706344].

[6] A. V. Belitsky and D. Müller, Phys. Lett. B486, 369 (2000) [hep$\mathrm{ph} / 0005028]$.

[7] A. V. Belitsky, D. Müller, L. Niedermeier and A. Schäfer, hep-ph/0004059.

[8] L. L. Frankfurt, P. V. Pobylitsa, M. V. Polyakov and M. Strikman, Phys. Rev. D60, 014010 (1999) [hep-ph/9901429].

[9] J. Breitweg et al. [ZEUS Collaboration], Eur. Phys. J. C6, 603 (1999) [hepex/9808020];

C. Adloff et al. [H1 Collaboration], Phys. Lett. B483, 23 (2000) [hepex/0003020].

[10] L. Frankfurt, A. Freund, V. Guzey and M. Strikman, Phys. Lett. B418, 345 (1998) [hep-ph/9703449];

K. J. Golec-Biernat, A. D. Martin and M. G. Ryskin, Phys. Lett. B456, 232 (1999) [hep-ph/9903327];

[11] M. G. Ryskin, hep-ph/9706505.

[12] M. Vänttinen and L. Mankiewicz, Phys. Lett. B434, 141 (1998) [hepph/9805338]; Phys. Lett. B440, 157 (1998) [hep-ph/9807287].

[13] L. Mankiewicz and G. Piller, Phys. Rev. D61, 074013 (2000) [hep$\mathrm{ph} / 9905287]$. 


\title{
A QCD Analysis of Polarised Parton Densities
}

\author{
Dilip Kumar Ghosh, Sourendu Gupta, \\ Tata Institute of Fundamental Research, Homi Bhabha Road, Bombay 400005, India \\ D. Indumathi* \\ The Institute of Mathematical Sciences, CIT Campus, Chennai 600113, India
}

\begin{abstract}
We present the results of QCD fits to global data on deep-inelastic polarised lepton-hadron scattering. It turns out that the data can be equally well-fitted with or without strongly broken $S U(2)$ flavour for the polarised sea densities. This can be tested in $W$ production at polarised RHIC. The data fails to pin down polarised singlet sea quark and gluon densities. We explore the uncertainties in detail and show that improvement in statistics, achievable at polarised $\mathrm{eRHIC}$ or HERA for measurement of $A_{1}$ at moderately low values of $x$, have large payoffs in terms of the improvement in measurement of gluon densities.
\end{abstract}

\section{INTRODUCTION}

A decade since the first longitudinally polarised e-p deep inelastic scattering (DIS) experiments [1] first precipitated the "proton spin crisis", many polarised DIS experiments [2-6] have reported measurements of the virtual photon asymmetry,

$$
A_{1}\left(x, Q^{2}\right)=\frac{g_{1}\left(x, Q^{2}\right)}{F_{1}\left(x, Q^{2}\right)}
$$

on different targets- $-p, D$ and ${ }^{3} \mathrm{He}$. What characterises these measurements is that they are all from fixed-target experiments, with a limited low- $x$ reach. Here, $x$ is the usual Bjorken scaling variable, $x=Q^{2} /(2 p \cdot q), Q^{2}=-q^{2}$, where $p$ and $q$ are the 4-momenta of the proton and the virtual photon respectively. The structure function of interest here is $g_{1}\left(x, Q^{2}\right)$, with information on $F_{1}$ (or rather, $F_{2}$ and $R$ ) coming from the already copious amount of data available from unpolarised DIS experiments, as well as other semi-inclusive measurements in $p-p$ collisions. Another polarised structure function of interest is that measured in polarised collisions when the proton (or hadron target) is polarised transverse to the beam direction: $g_{2}\left(x, Q^{2}\right)$. While the twist- 2 part of $g_{2}$ is believed to be determined by the $g_{1}$ structure function through the Wandzura-Wilczek sum rule [7], very little is known (either experimentally or theoretically) about its twist-3 component.

We present a parametrisation of polarised parton distributions from a QCD fit to currently available $g_{1}$ data [8] from polarised $e-p$ DIS. It will be seen that the data does not presently constrain the polarised singlet or gluon densities very well. The extent of $S U(2)$ flavour symmetry breaking in the sea is also not well determined. It will therefore be interesting to see if a polarised e-p collider experiment such as at HERA or eRHIC can improve our understanding of these densities. This is one of the main motivations of this talk.

Fig. 1 shows a set of input polarised densities that have been obtained by fitting to the following $A_{1}$ data:

- SMC collaboration [2]: data on proton and deuterium targets.

- E142 collaboration [3]: data on Helium ${ }^{3} \mathrm{He}$ (neutron) target.

- E143 collaboration [4]: data on proton and deuterium targets.

- E154 collaboration [5]: data on Helium ${ }^{3} \mathrm{He}$ (neutron) target.

- HERMES collaboration [6]: data on proton and Helium ${ }^{3} \mathrm{He}$ (neutron) targets.

The CTEQ parametrisation, CTEQ4 [9], was used to determine the denominator of the asymmetry, $F_{1}\left(x, Q^{2}\right)$. This was done to remove uncertainties due to differences in extraction of $g_{1}$ by different groups due to the use of different values for $F_{2}$ and $R$. For consistency as well as convenience in applying various constraints, the numerator of the asymmetry, that is, the polarised parton densities describing the structure function $g_{1}$, were also fitted to similar

- Talk presented at the eRHIC Summer Workshop. BNL, June 26-july 14th, 2000 
functional forms as the corresponding unpolarised densities. Also, the starting scale for evolution of the densities is the same for unpolarised and polarised caes: $Q_{0}^{2}=2.56 \mathrm{GeV}^{2}$. Hence, in terms of a parton description, we have

$$
g_{1}\left(x, Q^{2}\right)=\tilde{\mathcal{C}}_{q} \otimes \frac{1}{2} \sum_{f} e_{f}^{2}\left[\tilde{q}_{f}+\tilde{\bar{q}}_{f}\right]+\frac{1}{2}\left(\sum_{f} e_{f}^{2}\right) \tilde{\mathcal{C}}_{g} \otimes \tilde{g}
$$

which is a Mellin convolution of the quark $\left(\widetilde{q}_{f}\right)$, anti-quark $\left(\widetilde{\bar{q}}_{f}\right)$ and gluon $(\tilde{g})$ longitudinally polarised distributions with the known coefficient functions $\widetilde{\mathcal{C}}_{q, g}$. The index $f$ denotes flavour, and $e_{f}$ is the charge carried by the quark. The unpolarised structure function, $F_{1}$, is given by a similar formula in terms of the corresponding unpolarised densities and coefficient functions. We have used the $\overline{M S}$ scheme at NLO for our description of the densities. Hence, there is no gluonic contribution to the first moment of the $g_{1}$ structure function in this scheme.

We follow the parametrisation of CTEQ4 and write, for the input densities,

$$
\tilde{f}\left(x, Q_{0}^{2}\right)=a_{0} x^{a_{1}}(1-x)^{a_{2}}\left(1+a_{3} x^{a_{4}}\right)
$$

for all densities apart from $q_{3}=2(\bar{u}-\bar{d})$, which is parametrised as

$$
\widetilde{q}_{3}\left(x, Q_{0}^{2}\right)=a_{0} x^{a_{1}}(1-x)^{a_{2}}\left(1+a_{3} \sqrt{x}+a_{4} x\right) .
$$

We have made the choice that the large- $x$ behaviour of any polarised density is the same as that of the unpolarised density; in other words, the parameter $a_{2}$ is the same for corresponding polarised and unpolarised densities (this assumption is sometimes given the name "helicity retention property" [10]). For simplicity we have also equated the polarised and unpolarised values of $a_{4}$ when this parameter is a power.

Finally, at $Q_{0}^{2}$ we have extended some of the CTEQ assumptions for unpolarised parton densities to polarised. These include equating the values of $a_{1}$ for $\widetilde{V}_{u}, \widetilde{V}_{d}$ and $\tilde{q}_{3}$, taking $a_{4}=1$ for $\tilde{q}_{0}$, equating the values of $a_{2}$ for $\widetilde{q}_{0}$ and $\widetilde{q}_{3}$. We also retain the choice $\eta \equiv 2 \widetilde{s} /(\widetilde{\bar{u}}+\tilde{\bar{d}})=1 / 2$ in some of our fits, but let it vary in others. Although these assumptions seem overly restrictive, the quality of the data does not allow us to fit many of these parameters.

In respect our work is similar to that of [11]. However, our analysis differs in several ways. For one, some of the data we use is more recent than the older fits. More importantly, we relax some of the assumptions which needed to be made in analysing the older data. We allow for flavour asymmetry (that is, a non-zero $\widetilde{q}_{3}$ in the polarised sea quark densities, and let the first moment of the gluon density vary freely in the fit. The next-to-leading order fit in which flavour SU(2) is broken is denoted as NLO $\bar{S}$ while that in which $\mathrm{SU}(2)$ symmetry is retained is called NLO $S$. Both sets fit the existing $A_{1}$ data equally well; this is a sign that this asymmetry cannot distinguish the two cases. The best fits to $A_{1}$ data on $p,{ }^{3} \mathrm{He}(n)$, and $D$ corresponding to the NLO $\bar{S}$ input densities shown in Fig. 1 are shown in Figs. 2-4.

The uncertainty in gluon densities may seem puzzling in view of the fact that the $Q^{2}$-dependence of the structure function $g_{1}$ involves the gluon strongly. Unfortunately, errors on $g_{1}$ are large in the low- $x$ region, where the contribution of the gluons dominate, primarily because the asymmetry $A_{1}$ is small at low- $x$. This can be seen from the large errors on the allowed input polarised gluon density that still fits the $g_{1}$ data, as shown in Fig. 1 . There are equally large errors on the fits to the sea part of the singlet density, $\widetilde{q}_{0}$, as can be seen from the errors to the fit parameters for the NLO $\bar{S}$ and NLO S sets in Table I and II.

Note that our error bars are deliberately conservative, due to our neglect of systematic errors. Since systematic errors are as large as the statistical errors, the naive procedure of summing them in quadrature would have led us to believe that at NLO positive $\Gamma_{g}$ is allowed at $1.5 \sigma$.

This large uncertainty in $\widetilde{g}$ comes because the only constraint on gluon densities at present are the data on $Q^{2}$ variations of $g_{1}$. Furthermore, the data at $x<0.1$ are most effective in constraining $\widetilde{g}$, and in this range, the data have large errors. The question is how accurately does $Q^{2}$ evolution fix these densities.

We investigated this question quantitatively by generating fake data at $x<0.1$ from the NLO S set. The values of $A_{1}^{p}$ so generated were smeared randomly over a $10 \%$ band to simulate noise in the data, and error bars of $20-30 \%$ were assigned to each such data point. This faked set is meant to mimic data that could possibly come from a future polarised eRHIC or HERA experiment. We redid our fits with this faked data set replacing all data on $A_{1}^{p}$ for $x<0.1$. This data brings down the error bars in the parameter $a_{0}$ appearing in $\widetilde{g}$ by a factor of 4 , and improves the errors in $a_{1}$ by a factor of 2-3. The region of parameter space allowed by this fake data is shown as the grey patch in Fig. 5 . Taking data at $x<0.01$ or over a larger range of $Q^{2}$, both of which would be feasible at polarised eRHIC or HERA, would constrain $\widetilde{g}$ even better [12]. In fact, the errors at small- $x$ (in the region of overlap) on $g_{1}$ are expected to be statistically smaller at cRHIC than at HERA [13]. The ability of RHIC to fix the gluon densities is, of course, well 
appreciated. However, if DIS experiments at eRHIC can fix the gluon densities better, then polarised RHIC can be used as a discovery machine.

We add a short note on flavour asymmetry. The flavour non-singlet combinations of the charged-current (CC) structure function $g_{1}^{W}$, or the parity-violating structure function $g_{5}$ (proportional to $(\tilde{u}+\tilde{\bar{d}}+\widetilde{\bar{s}})$ or $(\tilde{u}-\tilde{\bar{d}}-\widetilde{\bar{s}})$ for $e^{-}-p$ scattering and to $(\tilde{\bar{u}}+\tilde{d}+\tilde{s})$ or $(\tilde{\bar{u}}-\tilde{d}-\tilde{s})$ for $e^{+}-p$ scattering at LO) will be sensitive to flavour non-singlet densities. However, polarised HERA with a larger energy $(\sqrt{s} \sim 300 \mathrm{GeV})$ than expected at eRHIC $(\sqrt{s} \sim 50-100 \mathrm{GeV})$ is more likely to make precise measurements of these structure functions. A possible flavour sensitive measurement can be made at polarised RHIC itself: it has been suggested [14] that $S U(2)$ flavour asymmetry in the sea be observed through two combinations of cross sections for production of $W^{ \pm}$in longitudinally polarised pp scattering-

$$
A_{\sigma, \delta}=\frac{\sigma\left(W^{+}, \uparrow \uparrow\right) \pm \sigma\left(W^{-}, \uparrow \uparrow\right)-\sigma\left(W^{+}, \uparrow \downarrow\right) \mp \sigma\left(W^{-}, \uparrow \downarrow\right)}{\sigma\left(W^{+}, \uparrow \uparrow\right) \pm \sigma\left(W^{-}, \uparrow \uparrow\right)+\sigma\left(W^{+}, \uparrow \downarrow\right) \pm \sigma\left(W^{-}, \uparrow \downarrow\right)}
$$

where $A_{\sigma}\left(A_{\delta}\right)$ is defined with the upper (lower) signs. At LO these asymmetries can be written as the ratio of certain combinations of polarised and unpolarised parton densities. At LO the asymmetry $A_{D Y}$ for Drell-Yan pairs can also be written in terms of the parton densities. At zero rapidity, $A_{D Y}$ is a function of $\sqrt{\tau}=M / \sqrt{s}$, where $M$ is the mass of the pairs and $\sqrt{s}$ is the center of mass energy of the colliding protons. For $W^{ \pm}$production at zero rapidity, the parton densities have to be evaluated at $M=M_{W}$, and hence $\sqrt{s}=M_{W} / \sqrt{\tau}$.

In Fig. 6 we have shown these asymmetries at zero rapidity as a function of $\sqrt{s}$, or equivalently of $\sqrt{\tau}$. We used our LO fits, described in detail in Ref. [8]. It is clear that at $\sqrt{s}$ appropriate to RHIC, the iso-triplet spin asymmetry, $A_{\delta}$, is best suited to distinguish the LO S densities from LO $\overline{\mathrm{S}}$. Experimentally studying the dependence of $A_{\delta}$ on $\sqrt{s}$ over even a limited range below $\sqrt{s}=500 \mathrm{GeV}$ would be very useful. $A_{D Y}$ is the least suitable measurement for making this distinction.

The sign and magnitude of $\tilde{g}$ can thus be fixed by various experiments at RHIC or in charm production measurements at HERA [15] or the COMPASS experiment in CERN [16].

In conclusion, we now have a better understanding of polarised DIS phenomena. However, a detailed understanding of the polarised sea densities (singlet quark and gluon) must await other/improved measurements of the spin asymmetry at low- $x$ values. A polarised HERA or eRHIC option can very well perform this task. Furthermore, measurements of the asymmetry in the iso-triplet part of $W^{ \pm}$production at $\mathrm{RHIC}$ are likely to be able to pin down the flavour content of the sea. Measurements at such future facilities for spin physics will thus nicely complement each other.

[1] M. J. Alguard et al. (E80), Phys. Rev. Lett. 37 (1976) 1261; G. Baum et al. (E130), Phys. Rev. Lett. 51 (1983) 1135; J. Ashman et al. (EMC), Phys. Lett. B 206 (1988) 364.

[2] B. Adeva et al. (SMC), Phys. Rev. D 58 (1998) 112001.

[3] P. L. Anthony et al. (E142), Phys. Rev. D 54 (1996) 6620.

[4] K. Abe et al. (E143), Phys. Rev. D 58 (1998) 112003.

[5] K. Abe et al. (E154), Phys. Rev. Lett. 79 (1997) 26.

[6] A. Airapetian et al. (HERMES), Phys. Lett. B 442 (1998) 484.

[7] S. Wandzura and F. Wilczek, Phys. Lett. B 72 (1977) 195.

[8] Dilip Kumar Ghosh, Sourendu Gupta, D. Indumathi, preprint hep-ph/0001287, to appear in Phys. Rev. D. 2000.

[9] H. L. Lai et al., Phys. Rev., D 55 (1997) 1280.

[10] F. E. Close and D. Sivers, Phys. Rev. Lett. 39 (1977) 1116; S. J. Brodsky and I. Schmidt, Phys. Lett. B 234 (1990) 114

[11] M. Glück et al., Phys. Rev. D 53 (1996) 4775;

T. Gehrmann and W. J. Stirling, Phys. Rev., D 53 (1996) 6100;

K. Abe et al. (E-154), Phys. Lett. B 405 (1997) 180;

C. Bourrely et al., Prog. Theor. Phys. 99 (1998) 1017;

G. Altarelli et al., Acta Phys. Polon. B 29 (1998) 145;

D. de Florian, O. Sampayo and R. Sassot, Phys. Rev., D 57 (1998) 5803;

L. E. Gordon, M. Goshtasbpour and G. P. Ramsey, Phys. Rev. D 58 (1998) 094017;

E. Leader, A. V. Sidorov and D. B. Stamenov, Phys. Rev. D 58 (1998) 114028;

Y. Goto et al., e-print hep-ph/0001046.

[12] A. De Roeck et al., Eur. Phys. J. C 6 (1999) 121. 
[13] W. Vogelsang, these proceedings.

[14] M. A. Doncheski et al., Phys. Rev. D 49 (1994) 3261;

H.-Y. Cheng, M.-L. Huang and C. F. Wai, Phys. Rev. D 49 (1994) 1272.

[15] T. Gehrmann, e-print hep-ph/9908500;

J. Feltesse and A. Schäfer, Proceedings of the workshop, "Future Physics at HERA", Hamburg, 1995/96, Eds. G. Ingelman, A. De Roeck, and R. Klanner, DESY (Hamburg, 1996).

[16] L. Schmitt (for the Compass Collaboration), Contribution at the International Conference on High Energy Physics, ICHEP, Vancouver, 1998. 


\begin{tabular}{cccccc}
\hline \hline density & $a_{0}$ & $a_{1}$ & $a_{2}$ & $a_{3}$ & $a_{4}$ \\
\hline$\widetilde{V}_{u}$ & $0.615_{-9}^{+7}$ & $-0.32( \pm 2)$ & $3.689^{b}$ & $12.2( \pm 2)$ & $0.873^{b}$ \\
$\widetilde{V}_{d}$ & $-0.61( \pm 2)$ & $-0.32^{a}$ & $4.247^{b}$ & $2.2( \pm 1)$ & $0.333^{b}$ \\
$\widetilde{q}_{0}$ & $0.009( \pm 9)$ & $-0.2_{-2}^{+\infty}$ & $8.041^{b}$ & $8( \pm 16)$ & $1.000^{b}$ \\
$\widetilde{q}_{3}$ & $-0.22^{c}$ & $-0.32^{a}$ & $8.041^{b}$ & $0^{b}$ & $7( \pm 5)$ \\
$\widetilde{g}$ & $-1.0_{-4}^{+3}$ & $-0.7_{-1}^{+2}$ & $4.673^{b}$ & $-5_{-2}^{+4}$ & $1.508^{b}$ \\
\hline \hline
\end{tabular}

TABLE I. The NLO $\bar{S}$ fits for the parameters in eqs. $(3,4)$ at $Q_{0}^{2}=2.56 \mathrm{GeV}^{2}$. The error estimates shown in the brackets apply to the last digit of the estimated value. In case of asymmetric errors, if one of the errors is zero it indicates that the parameter is at the limit of positivity. The parameters marked (a) are set equal to some other in the same column, (b) are fixed to the value taken by the unpolarised densities, and (c) are fixed by the Bjorken sum rule.

\begin{tabular}{cccccc}
\hline \hline density & $a_{0}$ & $a_{1}$ & $a_{2}$ & $a_{3}$ \\
\hline$\widetilde{V}_{u}$ & $1.74( \pm 1)$ & $-0.149_{-3}^{+4}$ & $3.689^{b}$ & $3.91_{-3}^{+6}$ & $0.873^{b}$ \\
$\widetilde{V}_{d}$ & $-0.75^{c}$ & $-0.149^{a}$ & $4.247^{b}$ & $1.6( \pm 1)$ & $0.333^{b}$ \\
$\widetilde{q}_{0}$ & $-0.26( \pm 2)$ & $-0.08( \pm 4)$ & $8.041^{b}$ & $6.5( \pm 7)$ & $1.000^{b}$ \\
$\widetilde{g}$ & $-0.3_{-0}^{+1}$ & $-0.6_{-2}^{+3}$ & $4.673^{b}$ & $-17_{-0}^{+15}$ & $1.508^{b}$ \\
\hline \hline
\end{tabular}

TABLE II. The NLO S fits for the parameters in eq. (3) at $Q_{0}^{2}=2.56 \mathrm{GeV}^{2}$. Asymmetric errors and superscripts on the numbers have the same meaning as in Table I. 


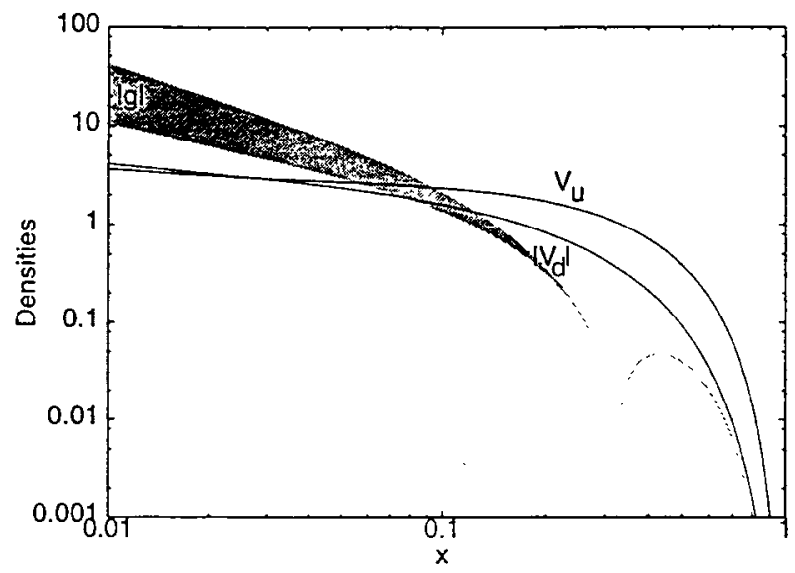

FIG. 1. The absolute values of the polarised valence quark densities are shown along with the range of allowed $|\widetilde{g}|$ in the NLO $\overline{\mathrm{S}}$ set. The gray band showing this uncertainty is the band enclosed by the densities obtained at. points $A$ and $\mathrm{B}$ marked in Fig. 5.

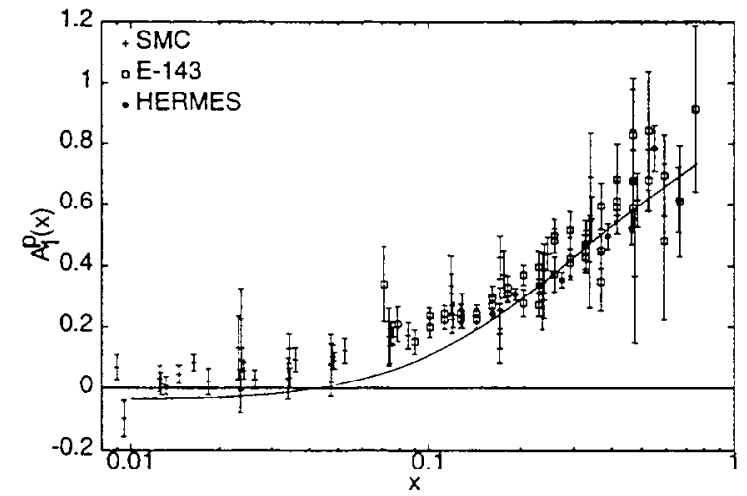

FIG. 2. Data and fits for $A_{1}^{p}$. The data are at different $Q^{2}$, but the curve is the asymmetry from NLO $\overline{\mathrm{S}}$ set ralculated at fixed $Q_{0}^{2}=5 \mathrm{GeV}^{2}$.

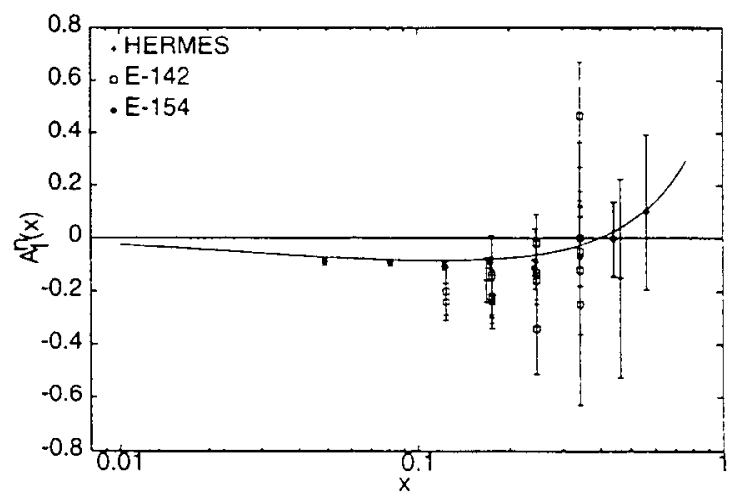

FIG. 3. Data and fits for $A_{1}^{r}$. The data are at different $Q^{2}$, but the curve is the asymmetry from NLO $\bar{S}$ set calculated at fixed $Q_{0}^{2}=5 \mathrm{GeV}^{2}$. 


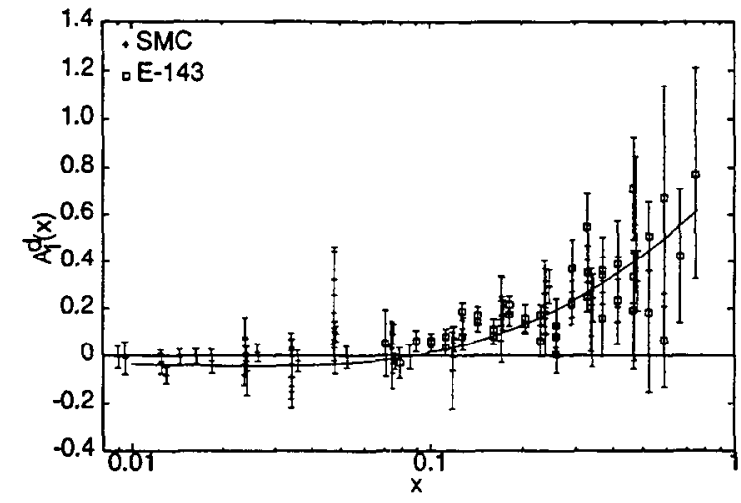

FIG. 4. Data and fits for $A_{1}^{d}$. The data are at different $Q^{2}$, but the curve is the asymmetry from NLO $\overline{\mathrm{S}}$ set calculated at fixed $Q_{0}^{2}=5 \mathrm{GeV}^{2}$.

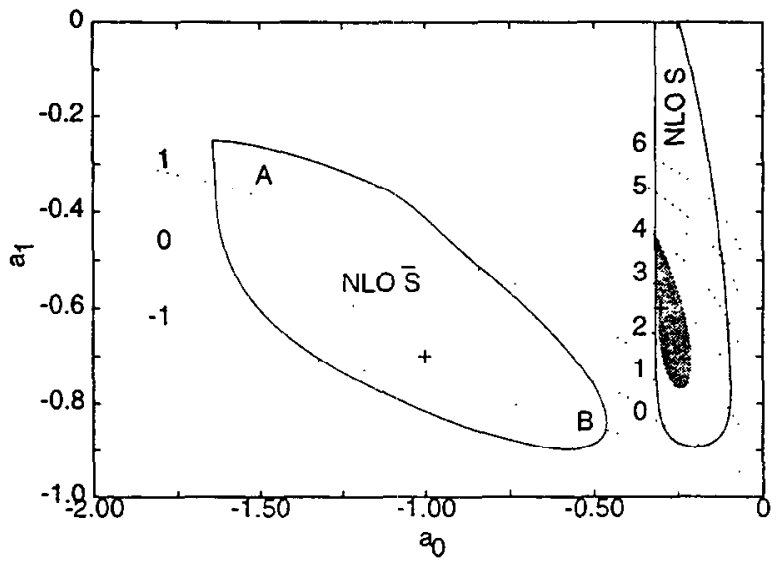

FIG. 5. The covariance of the fitted parameters $a_{0}$ and $a_{1}$ for $\widetilde{g}$ for the two NLO sets with $a_{3}$ kept at their respective best fit values. The crosses show the best fit points, and full lines are the contours enclosing the $68 \%$ confidence limits. The reference points $\mathrm{A}$ and $\mathrm{B}$ are used to quantify the variation in gluon densities in Fig. 1. The shaded area in NLO S indicates the errors on the fits using the faked data.

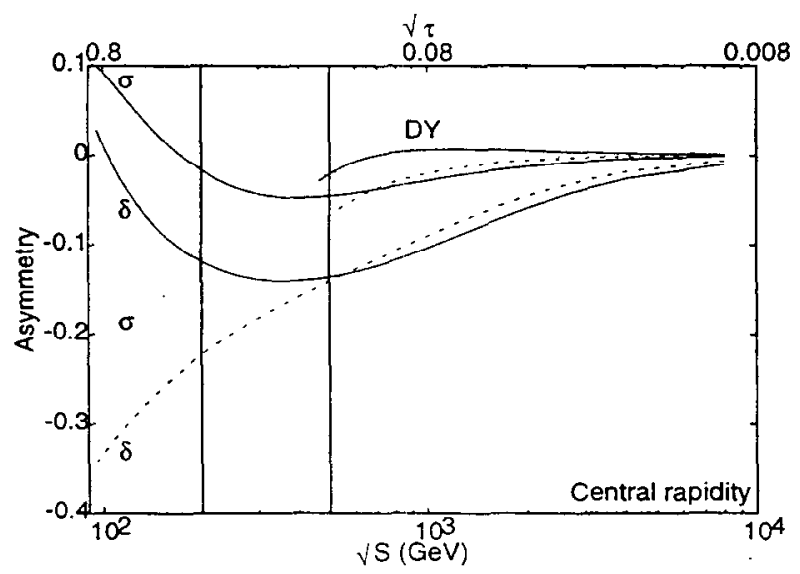

FIG. 6. The asymmetries $A_{D Y}$ and $A_{\sigma}$ and $A_{\delta}$ (defined in eq. 5) computed at LO. The full lines are obtained with LO $\overline{\mathrm{S}}$ and the dotted lines with LO S [8]. The bottom scale is for $A_{\delta, \sigma}$ as a function of $\sqrt{s}$ and that at the top for $A_{D Y}$ as a functions of $\sqrt{\tau}$. The vertical band marks out the range $200 \mathrm{GeV} \leq \sqrt{s} \leq 500 \mathrm{GeV}$. 


\title{
Initial Gluon Energy and Multiplicity in Heavy-ion Collisions
}

\author{
or \\ Exciting a Colored Glass \\ Condensate* \\ R. Venugopalan and A.K. \\ eRHIC Meeting at BNL, 7.7.00
}

- Reminder of MV model.

- Lattice formulation.

- Energy density estimates.

- Gluon multiplicity estimates.

- Discussion.

·NPB 557 (1999) 237; PRL 84 (2000) 4309; hep-ph/0004116; hep-ph/0007? 


\section{Reminder of McLerran-Venugopalan model}

Partons in a nucleus are separated into the high- $x$ (valence + hard sea) and low- $x$ parts. The latter part corresponds to central rapidity in HIC where QGP is supposed to form.

High- $x$ partons are considered recoilless sources of color charge. For a large, Lorcntz-contracted nucleus this color charge distribution is Gaussian in the transverse plane.

$$
P([\rho]) \propto \exp \left[-\frac{1}{2 g^{4} \mu^{2}} \int \mathrm{d}^{2} r_{t} \rho^{2}\left(r_{t}\right)\right] .
$$

This color charge is static in the transverse plane.

A nucleus is considered infinitely thin in the longitudinal direction. This assumption can be relaxed if necessary.

$\mu$ is a dimensional parameter which determines the color charge density. With the inclusion of semi-hard partons

$$
\begin{aligned}
& \mu^{2}=\frac{A^{1 / 3}}{\pi r_{0}^{2}} \int_{x_{0}}^{1} \mathrm{~d} x\left(\frac{1}{2 N_{c}} q\left(x, Q^{2}\right)+\frac{N_{c}}{N_{c}^{2}-1} g\left(x, Q^{2}\right)\right) \\
& \left(x_{0} \equiv Q / \sqrt{s}\right) .
\end{aligned}
$$

Consider a nuclear collision [Kovner, Mclerran, Weigert]. Solve equations of motion for low- $x$ fields in the random color-charge background:

$$
D_{\mu} F_{\mu \nu}=J_{\nu},
$$

where. for an infinitely thin nucleus.

$$
J_{\nu}=\sum_{1,2} \delta_{\nu . \pm} \delta\left(x_{\mp}\right) \rho_{1.2}\left(r_{t}\right) .
$$


For a single nucleus the solution is a pure gauge:

$$
A_{ \pm}=0, \quad A_{\perp}=i \mathrm{e}^{i \Lambda\left(r_{t}\right)} \partial_{\perp} \mathrm{e}^{-i \Lambda\left(r_{t}\right)} \theta\left(x_{\mp}\right),
$$

where

$$
\nabla^{2} \Lambda=\rho .
$$

(note $\theta\left(x_{\mp}\right)$ ensuring causality).

It is necessary to match solutions in 3 regions, differing by their causal relation to the nuclei.

In region 3 (after the collision) the field strength does not vanish. Using $A_{\tau}=0$ and $\nabla \cdot A_{\perp}=0$ at $\tau=0,[\mathrm{KMW}]$ arrive, perturbatively, at the multiplicity per unit rapidity

$$
N_{k_{\perp}} \propto\left(\frac{\alpha_{s}^{3 / 4} \mu}{k_{\perp}}\right)^{4} \ln \left(\frac{k_{\perp}}{\alpha_{s} \mu}\right) .
$$

This resembles a high-temperature theory (assuming weak coupling):

- $T \longrightarrow \alpha_{s}^{3 / 4} \mu$

- $k \ll T \longrightarrow k_{\perp} \ll \alpha_{s}^{3 / 4} \mu$ (classical physics)

- $k \sim g^{2} T \longrightarrow k_{\perp} \sim \alpha_{s} \mu$ (non-perturbative domain, KMW not valid).

We need a non-perturbative tool for soft low- $x$ modes! 


\section{Lattice formulation}

The Hamiltonian formalism is better suited for numerical work. In the continuum [Makhlin]

$$
H=\frac{\tau}{2} \int \mathrm{d} \eta \mathrm{d}^{2} r_{t}\left[p^{\eta} p^{\eta}+\frac{1}{\tau^{2}} p^{r} p^{r}+\frac{1}{\tau^{2}} F_{\eta r} F_{\eta r}+F_{x y} F_{x y}\right] .
$$

For "perfect pancake" nuclei we only consider boost-invariant configurations. Hence

$$
A_{r}\left(\tau, \eta, \vec{r}_{t}\right)=A_{r}\left(\tau, \vec{r}_{t}\right), \quad A_{\eta}\left(\tau, \eta, \vec{r}_{t}\right)=\Phi\left(\tau, \vec{r}_{t}\right)
$$

(this resembles a finite- $\mathrm{T}$ dimensional reduction: an adjoint scalar emerges).

Per unit rapidity

$$
H=\frac{\tau}{2} \int \mathrm{d}^{2} r_{t}\left[p^{\eta} p^{\eta}+\frac{1}{\tau^{2}} E_{r} E_{r}+\frac{1}{\tau^{2}}\left(D_{r} \Phi\right)\left(D_{r} \Phi\right)+F_{x y} F_{x y}\right] .
$$

Discretize on a $2 \mathrm{~d}$ lattice

$$
\begin{aligned}
H_{L}= & \frac{1}{2 \tau} \sum_{l} E_{l} E_{l}+\tau \sum_{\square}\left(1-\frac{1}{N_{c}} \Re \operatorname{Tr} U_{\square}\right) \\
& +\frac{\tau}{2} \sum_{j} p_{j} p_{j}+\frac{1}{4 \tau} \sum_{j, n} \operatorname{Tr}\left(\Phi_{j}-U_{j, n} \Phi_{j+n} U_{j, n}^{\dagger}\right)^{2}
\end{aligned}
$$

and solve (numerically) the resulting equations of motion for $x_{ \pm}>$ 0 .

Interested in soft modes $\longrightarrow$ use classical approximation. 
Just as in the continuum

- Average over the static color charge

- Determine initial conditions by matching

Relation to the continuum physics

3 dimensional quantities occur in the classical lattice theory:

- $g^{2} \mu$ only in this combination

- $L$, the linear size of a nucleus

- $a$, the lattice cuttoff

In the units of $a$, in the continuum limit $g^{2} \mu \rightarrow 0, L \rightarrow \infty$, but $g^{2} \mu L$ is constant.

For any well-defined $P$ of dimension $d$

$$
P=\left(g^{2} \mu\right)^{d} f_{P}\left(g^{2} \mu L\right),
$$

where $f_{P}\left(g^{2} \mu L\right)$ contains all the non-trivial physical information.

- $\mathrm{RHIC}-\mu \leq 0.5 \mathrm{GeV}$

- $\mathrm{LHC}-\mu \leq 1 \mathrm{GeV}$

- Nuclear size: for $A=200$ and p.b.c. $L=11.5 \mathrm{fm}$. 


\section{Energy density estimates}

Goal: determine $d E / L^{2} / d \eta$.

$$
d E / L^{2} / d \eta=f_{E}\left(g^{2} \mu L\right)\left(g^{2} \mu\right)^{3} / g^{2}
$$

$\longrightarrow$ find $f\left(g^{2} \mu L\right)$ numerically.

Estimates for RHIC and LHC: assume $g=2, \mu=0.5 \mathrm{GeV}(1.0$ $\mathrm{GeV}$ ) for RHIC (LHC), $L=11.6 \mathrm{fm}$ for Au.

Then

- $g^{2} \mu L \approx 120$ for RHIC

- $g^{2} \mu L \approx 240$ for LHC

Also: assume $N_{c}$ dependence as $\left(N_{c}^{2}-1\right) / N_{c}$ (A. Mueller). Estimate initial volumes using $\tau_{D}$ and obtain:

- $\varepsilon^{R H I C} \approx 66.5 \mathrm{GeV} / \mathrm{fm}^{3}$

- $\varepsilon^{L H C} \approx 1300 \mathrm{GeV} / \mathrm{fm}^{3}$.

Corresponding to the total energy

- $E_{T}^{R H I C} \approx 2700 \mathrm{GeV}$

- $E_{T}^{L H C} \approx 25000 \mathrm{GeV}$

Adjusting for loop corrections:

- $E_{T}^{R H I C} \approx 5400 \mathrm{GeV}$ (Kajantie $E_{T}^{R H I C}=2500 \mathrm{GeV}$ )

- $E_{T}^{L H C} \approx 50000 \mathrm{GeV}$ (Kajantie $E_{T}^{L I C}=12000 \mathrm{GeV}$ ) 


\section{Gluon multiplicity estimates}

Employ 2 methods to estimate the gluon number, each extrapolating a definition of the particle number from a free theory:

$$
H_{f}=\frac{1}{2} \sum_{k}\left(|\pi(k)|^{2}+\omega^{2}(k)|\phi(k)|^{2}\right) .
$$

1. In a free theory

$$
n(k)=\omega(k)\left\langle|\phi(k)|^{2}\right\rangle=\sqrt{\left\langle|\phi(k)|^{2}|\pi(k)|^{2}\right\rangle} .
$$

Use this expression with fields and momenta determined in the Coulomb gauge. As a by-product, determine also the dispersion relation

$$
\omega(k)=\sqrt{\frac{\left\langle|\pi(k)|^{2}\right\rangle}{\left\langle|\phi(k)|^{2}\right\rangle}} .
$$

2. If a free field is subject to relaxation (cooling)

$$
\partial_{t} \phi(x)=-\partial H / \partial \phi(x)
$$

then

$$
N=\sqrt{\frac{8}{\pi}} \int_{0}^{\infty} \frac{\mathrm{d} t}{\sqrt{t}} V(t)
$$

where $V$ is the potential part of $H$. Generalize to full interactive $V$.

Assuming (as for the energy) the $\left(N_{c}^{2}-1\right) / N_{c}$ dependence, we obtain (from the relaxation method).

- $\mathrm{d} N / \mathrm{d} \eta_{\mathrm{RHIC}} \approx 10^{3}$

- $\mathrm{d} N / \mathrm{d} \eta_{\mathrm{LHC}} \approx 4300$ 


\section{Discussion}

1. We have at our disposal a fully non-perturbative tool for low- $x$ physics in HIC.

2. We have made contact with the perturbative regime (for $k_{\perp} \gg$ $\left.\alpha_{s} \mu\right)$.

3. First results: estimates of the initial energy in the RHIC and LHC regimes.

4. Further results: estimates of gluon multiplicities in the RHIC and $\mathrm{LHC}$ regimes.

5. Obvious way to improve: $\mathrm{SU}(2) \longrightarrow \mathrm{SU}(3)$.

6. Possible way to improve: relax the exact boost invariance.

7. Other interesting observables? 


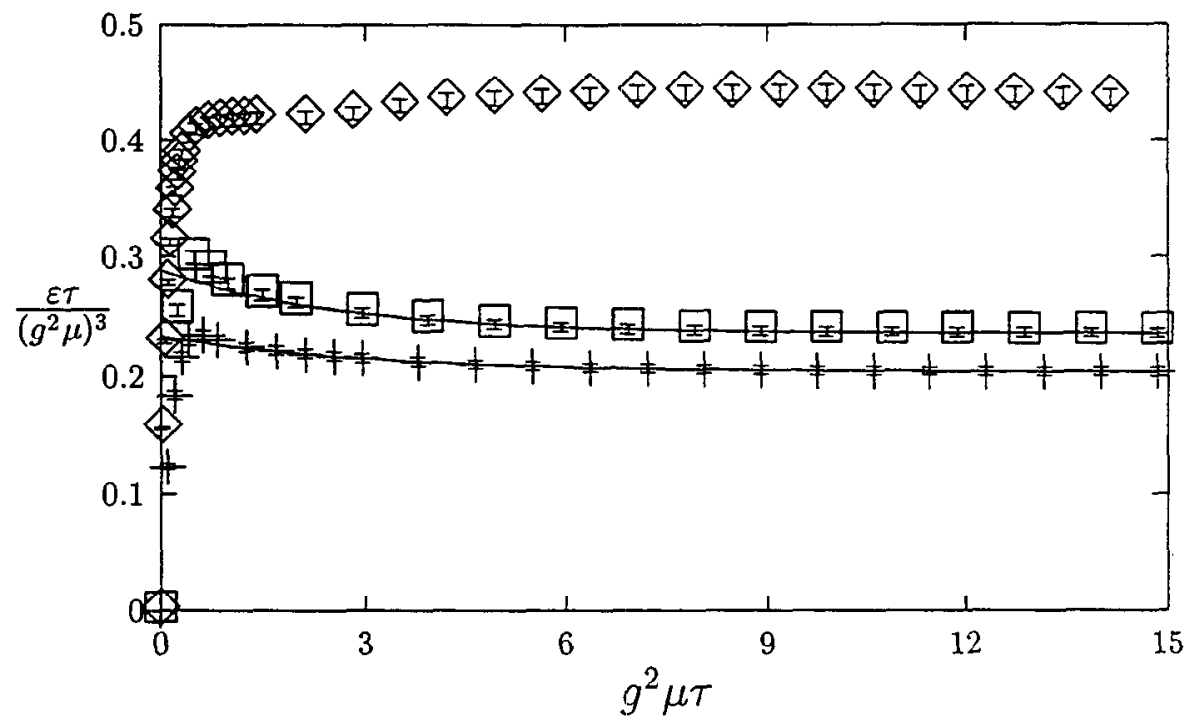

Figure 1: $\varepsilon \tau /\left(g^{2} \mu\right)^{3}$ as a function of $g^{2} \mu \tau$ for $g^{2} \mu L=5.66$ (diamonds), 35.36 (pluses) and 296.98 (squares). Both axes are in dimensionless units. Note that $\varepsilon \tau=0$ at $\tau=0$ for all $g^{2} \mu L$. The lines are exponential fits $\alpha+\beta e^{--\gamma \tau}$ including all points beyond the peak. 

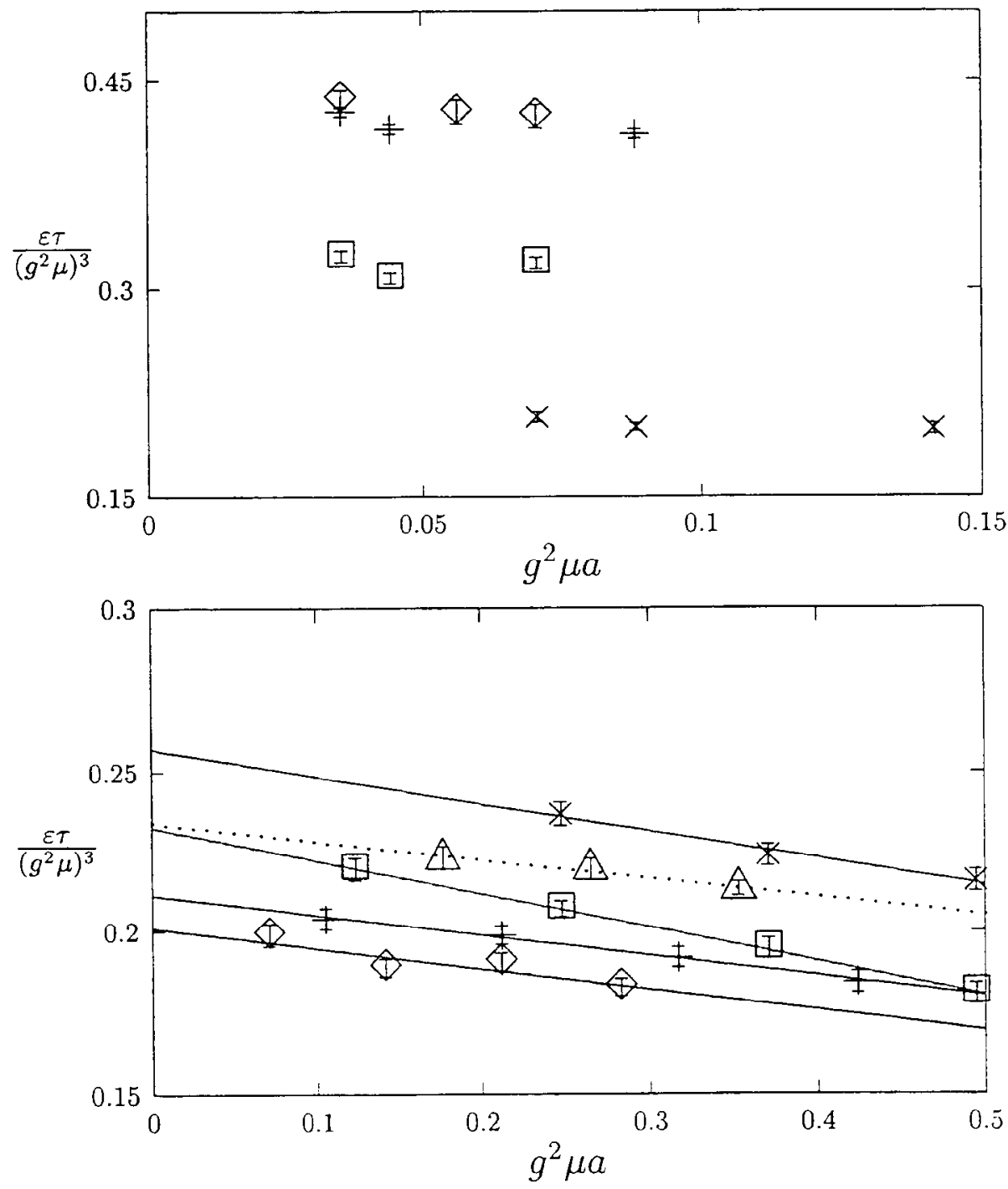

Figure 2: $\varepsilon \tau /\left(g^{2} \mu\right)^{3}$ as a function of $g^{2} \mu a$. The points in the upper plot correspond to $g^{2} \mu L=$ 5.66 (diamonds), 8.84 (pluses), 17.68 (squares), and 35.36 (x's). The lower plot has $g^{2} \mu L=70.7$ (diamonds), 106.06 (pluses), 148.49 (squares), 212.13 (triangles) and 296.98 (x s). Lines in the lower plot are fits of form $a-b \cdot x$. The $g^{2} \mu a$ ranges are different in the two halves. The points in the upper half are typically closer to the continuum limit. 


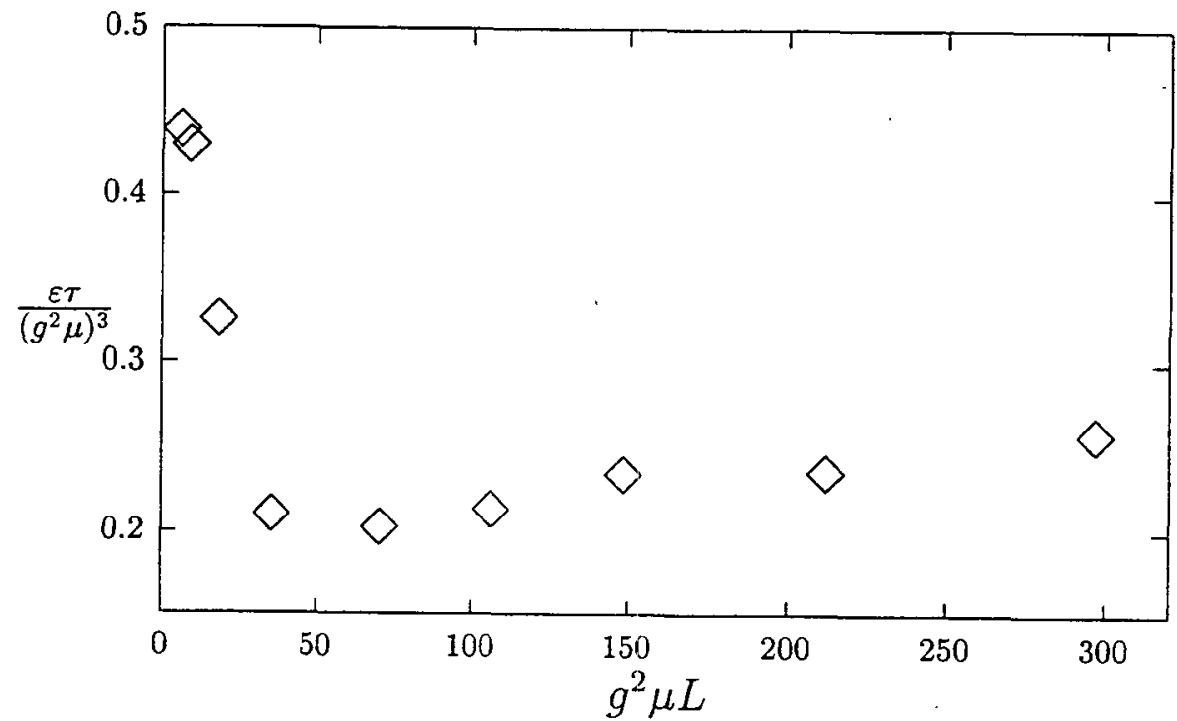

Figure 3: $\varepsilon \tau /\left(g^{2} \mu\right)^{3}$ extrapolated to the continuum limit: $f$ as a function of $g^{2} \mu L$. The error bars are smaller than the plotting symbols.

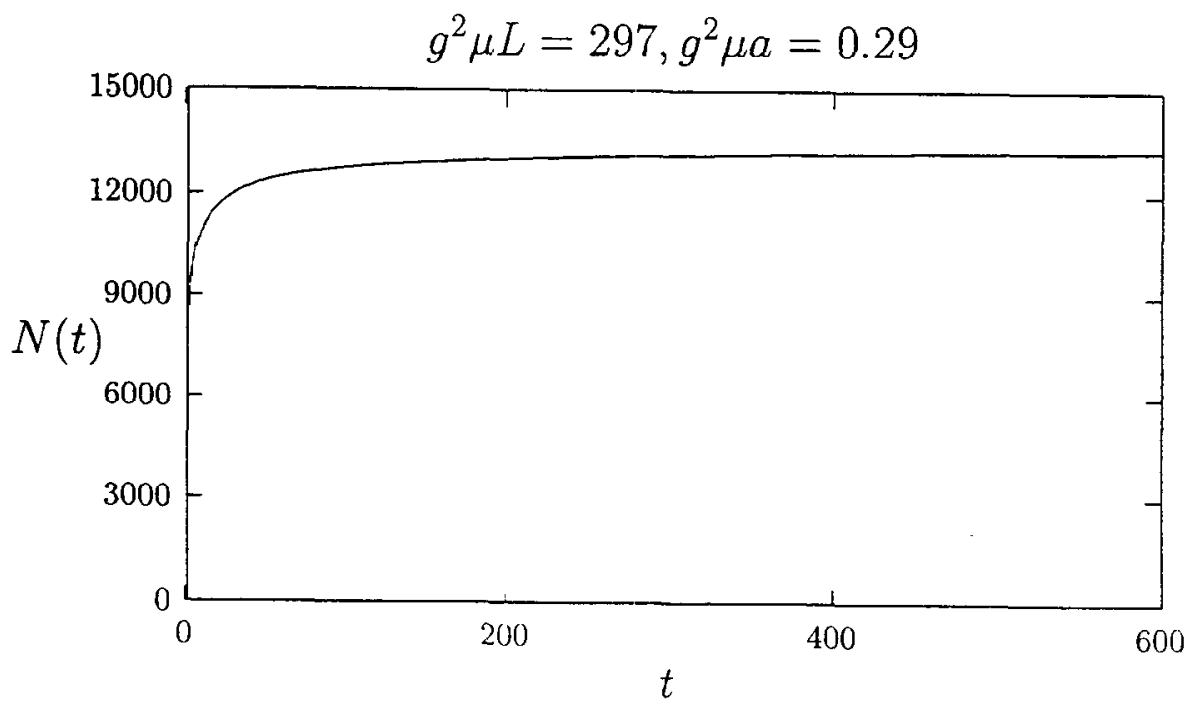



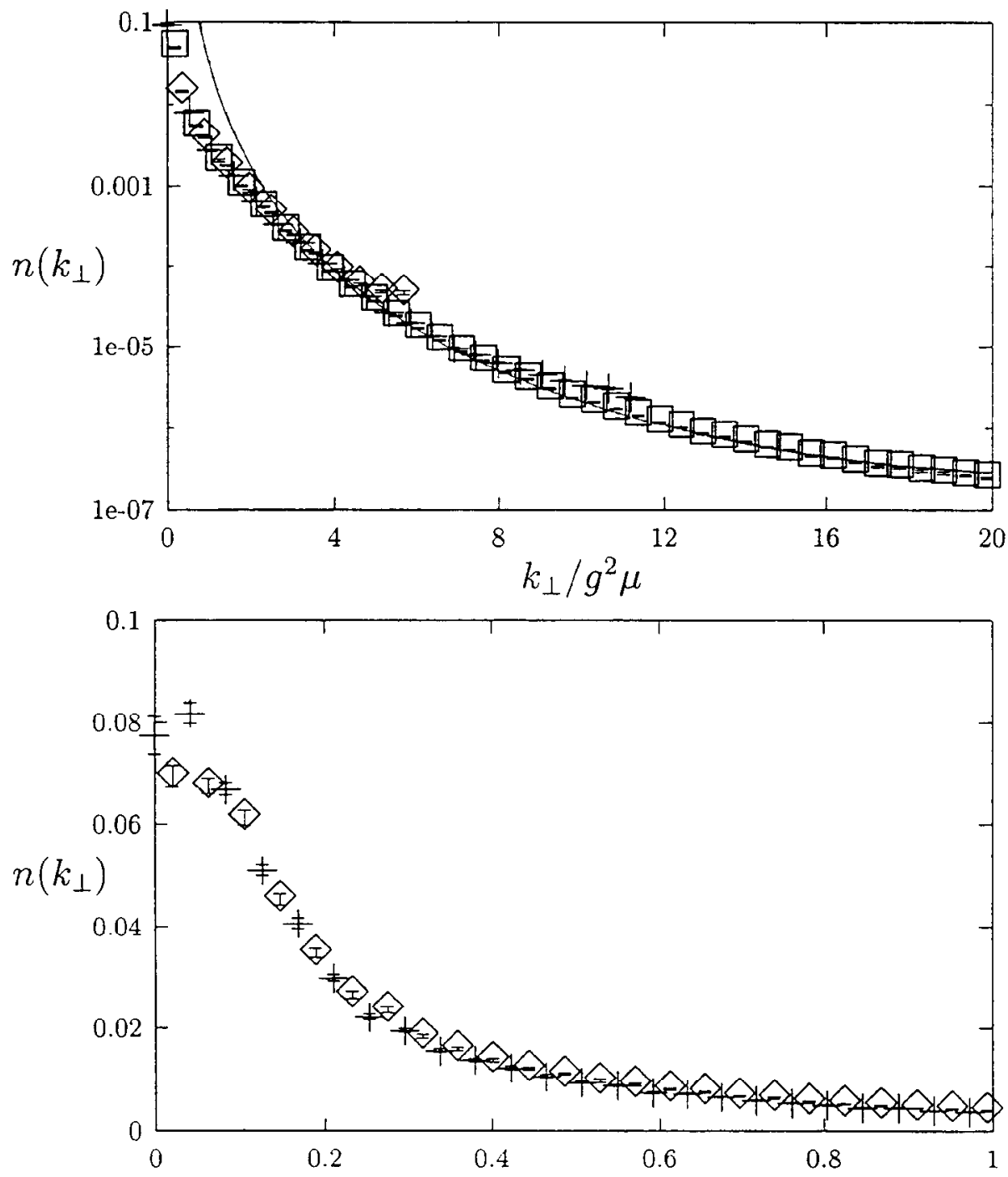

Figure 4: Upper: gluon distribution function for $g^{2} \mu L=35.5$ and the values 0.138 (squares), 0.276 (plusses), and 0.552 (diamonds) of $g^{2} \mu a$. The gluon momentum $k$ is in units of $g^{2} \mu$. The solid line is a fit of the lattice perturbative expression to the high-momentum part of the $g^{2} \mu a=0.138$ data. Lower: gluon distribution function at soft gluon momentum $k$ for $g^{2} \mu L=148.5$ (plusses) and $g^{2} \mu L=297$ (diamonds), both at $g^{2} \mu a=0.29$. 


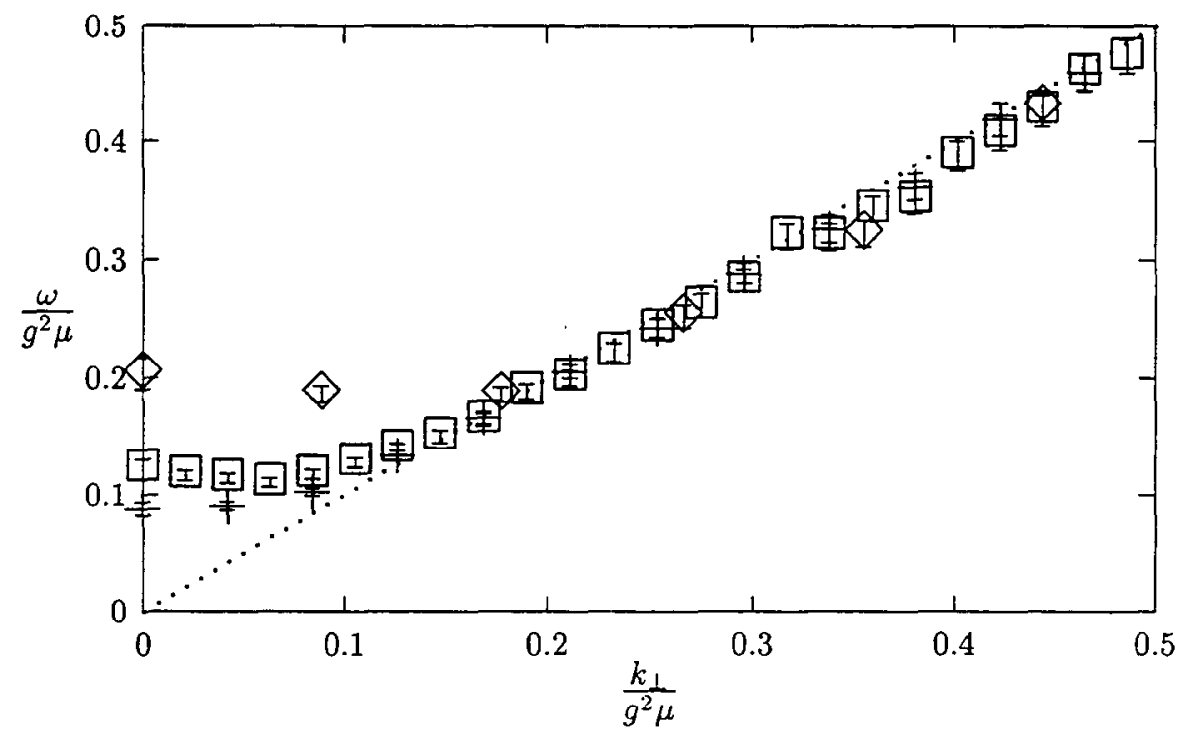

Figure 5: Gluon dispersion relation at soft momenta for $g^{2} \mu L=70, g^{2} \mu L=148.5$ and $g^{2} \mu L=$ 297.

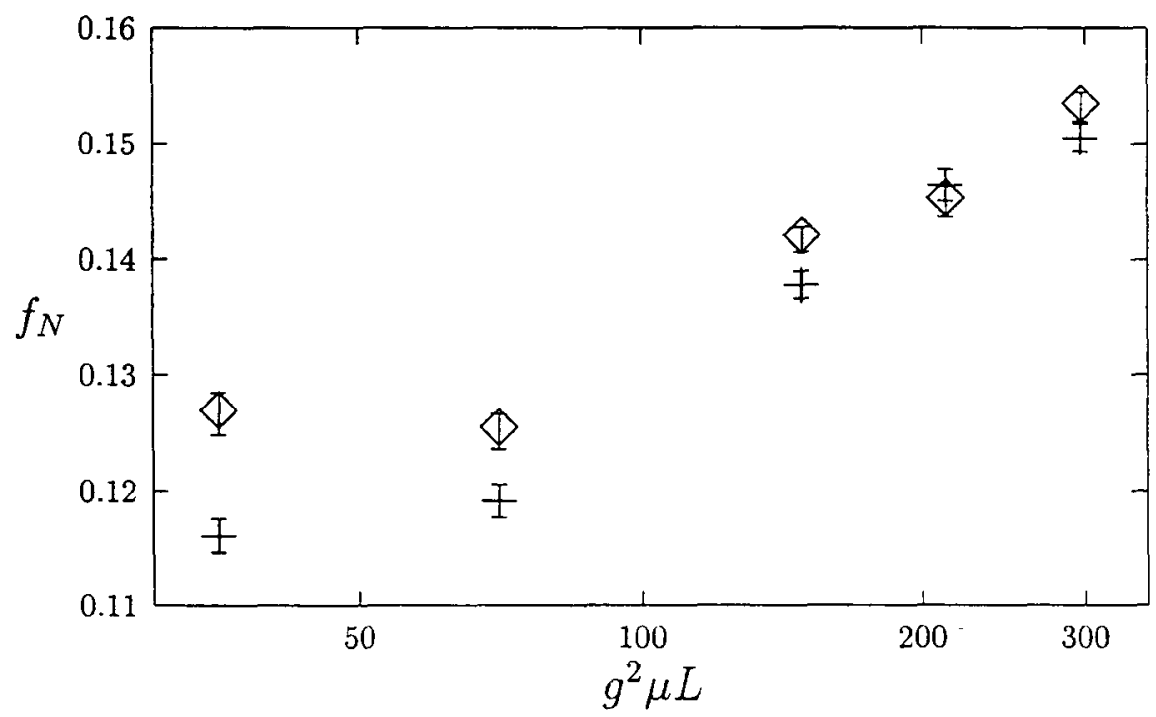

Figure 6: Total gluon number per unit area per unit rapidity in units of $g^{2} \mu$ as a function of $g^{2} \mu L$. 


\title{
Deeply virtual Compton scattering at small $x$
}

\author{
IAN BALITSKY
}

Physics Department, Old Dominion University, Norfolk, VA 23529

\begin{abstract}
We calculate the cross section of the deeply virtual Compton scattering at large energies and intermediate momentum transfers.

In recent years the study of the deeply virtual Compton scattering (DVCS) became one of the most popular topics in QCD due to the fact that it is determined by skewed parton distributions [1-3] which generalize usual parton densities introduced by Feynman. These new probes of the nucleon structure are accessible in exclusive processes such as DVCS and potentially they can give us more information than the traditional parton densities. Here we consider the small-x DVCS where the energy of the incoming virtual photon $E$ is very large in comparison to its virtuality $Q^{2}$.

To be specific, we calculate the DVCS amplitude in the region

$$
s \gg Q^{2} \gg-t \gg m^{2}
$$

where $s=2 m E, m$ is the nucleon mass, and $t$ is the momentum transfer. The DVCS in this region is a semihard processes which can be described by the BFKL pomeron [4]. It turns out that at large momentum transfer the coupling of the BFKL pomeron to the nucleon is essentially equal to the Dirac form factor of the nucleon $F_{1}(t)$, so the DVCS amplitude in the region (1) can be calculated without any model assumptions. The results obtained in this region can be used for the estimates of the amplitude at experimentally accessible energies where one or more conditions in Eq. (1) are relaxed. To be spccific, we have in mind the HERA kinematics where $x \sim 10^{-2} \div 10^{-4}, Q^{2} \geq 6 \mathrm{GeV}^{2}$, and $-t \sim 1 \div 5 \mathrm{GeV}^{2}$ [5]. Since there are only model predictions for the small-x DVCS in current literature [6],
\end{abstract}


even the approximate calculations of the cross section in QCD are very timely.

Similarly to the case of deep inelastic scattering (DIS), the amplitude of DVCS is determined by the matrix element [7]

$$
H^{A B}=i e_{\nu}^{A} e_{\mu}^{B} \int d z e^{i q^{\prime} z}\left\langle p^{\prime}\left|T\left\{j^{\mu}(z) j^{\nu}(0)\right\}\right| p\right\rangle
$$

where $q, p$ and $q^{\prime}, p^{\prime}$ are the initial and the final momenta of the photon and the nucleon, respectively. The momentum transfer is defined as $r=p^{\prime}-p$. Since $Q^{2}=-q^{2}$ is large we can use perturbation theory for the hard part of the DVCS process [8] [9]. The typical diagram for the DVCS amplitude in the lowest order in perturbation theory is shown in Fig.1 We will calculate at first the imaginary part of the amplitude $V^{A B}=\frac{1}{\pi} \operatorname{Im} T^{A B}$. Let

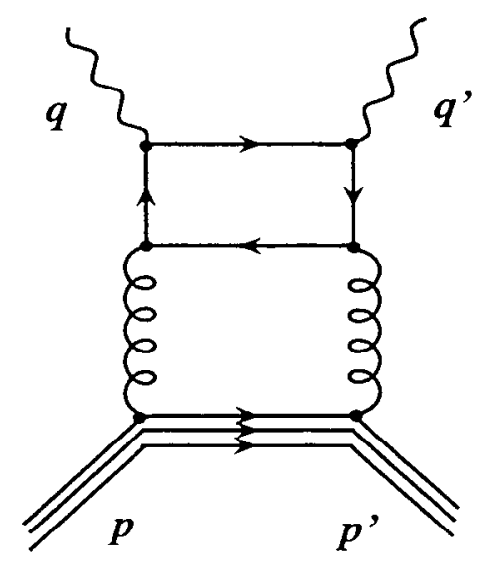

FIG. 1. A typical Feynman diagram for the high-energy $\gamma^{*} p \rightarrow \gamma p$ scattering

us define the light-like vectors $p_{1}=q^{\prime}, p_{2}=p^{\prime}-\frac{m^{2}}{s} p_{1}$, then

$$
\begin{aligned}
& q=p_{1}\left(1-\frac{r_{\perp}^{2}}{s}\right)-x p_{2}-r_{\perp} \\
& p=p_{2}(1+x)+\frac{m^{2}+r_{\perp}^{2}}{s} p_{1}+r_{\perp} \quad q^{\prime}=p_{1} \\
&
\end{aligned}
$$

where $x \equiv \frac{Q^{2}+t}{s} \simeq \frac{Q^{2}}{s}=x_{B j}$ and $t \simeq-r_{\perp}^{2}$ at large energies. It is well known that at high energies the Feynman integral for the imaginary part of the diagram shown in Fig. 1 factorizes into a product of two "impact factors" integrated with two-dimensional propagators 


$$
V^{A B}=\frac{2 s}{\pi} g^{4}\left(\sum e_{q}^{2}\right) \int \frac{d^{2} k_{\perp}}{4 \pi^{2}} \frac{1}{k_{\perp}^{2}} \frac{1}{(r+k)_{\perp}^{2}} I\left(k_{\perp}, r_{\perp}\right) I_{N}\left(k_{\perp}, r_{\perp}\right)
$$

where $\left(\sum e_{q}^{2}\right)$ is the sum of squared charges of active flavors $(u, d, s$, and possibly $c)$.

The photon impact factor can be easily calculated in the leading order in perturbation theory [10]. The impact factor for the proton which decribes the pomeron-nucleon coupling cannot be calculated perturbatively. However, it is possible to demonstrate [10] that at high momenta $k_{\perp}^{2} \gg m^{2}$ this impact factor reduces to

$$
I_{N}\left(k_{\perp}, r_{\perp}\right) \stackrel{k_{\perp}^{2}}{\stackrel{\gg m^{2}}{=}} F_{1}^{p+n}(t)
$$

where $F_{1}^{p+n}(t)$ is the sum of the proton and neutron Dirac form factors. The characteristic transverse momenta in our gluon loop are large so the estimate (5) is sufficient for our purposes. Substituting the nucleon impact factor (5) into Eq. (4) we obtain

$$
\begin{aligned}
& V^{A B}= \\
& \frac{2}{x}\left(\frac{\alpha_{s}}{\pi}\right)^{2}\left(\sum_{\text {flavors }} e_{q}^{2}\right) F_{1}^{p+n}(t) \\
& \left(\left(e^{A}, e^{B}\right)_{\perp}\left(\frac{1}{2} \ln ^{2} \frac{Q^{2}}{|t|}+2\right)-\left(e^{A}, e^{B}\right)_{\perp}+\frac{2}{r_{\perp}^{2}}\left(e^{A}, r\right)_{\perp}\left(e^{B}, r\right)_{\perp}+O\left(t / Q^{2}\right)\right)
\end{aligned}
$$

for the transverse polarizations. We neglect the longitudinal amplitude $V^{L B}$ since is twistsuppressed as $\frac{\sqrt{|t|}}{Q}$ in comparison to the transverse amplitude (6).

In the next two orders in perturbation theory the most important diagrams are those of the type shown in Fig. $2^{*}$. As we shall see below, the integrals over transverse momenta converges at $\left|k_{\perp}\right| \gg m$ so we can again use the approximation (5) for the nucleon impact factor. Calculation of this diagrams in the leading log approximation yields [10]

$$
\begin{aligned}
& V^{A B}= \\
& \frac{2}{x}\left(\frac{\alpha_{s}(Q)}{\pi}\right)^{2}\left(\sum_{\text {flavors }} e_{q}^{2}\right) F_{1}^{p+n}(t)\left[\left(e^{A}, e^{B}\right)_{\perp} v+\left(\frac{2}{r_{\perp}^{2}}\left(e^{A}, r\right)_{\perp}\left(e^{B}, r\right)_{\perp}-\left(e^{A}, e^{B}\right)_{\perp}\right) v^{\prime}\right]
\end{aligned}
$$

\footnotetext{
*Actually, these diagram give the total contribution in LLA if one replaces the three-gluon vertex
} in Fig. 2 by the effective Lipatov's vertex 

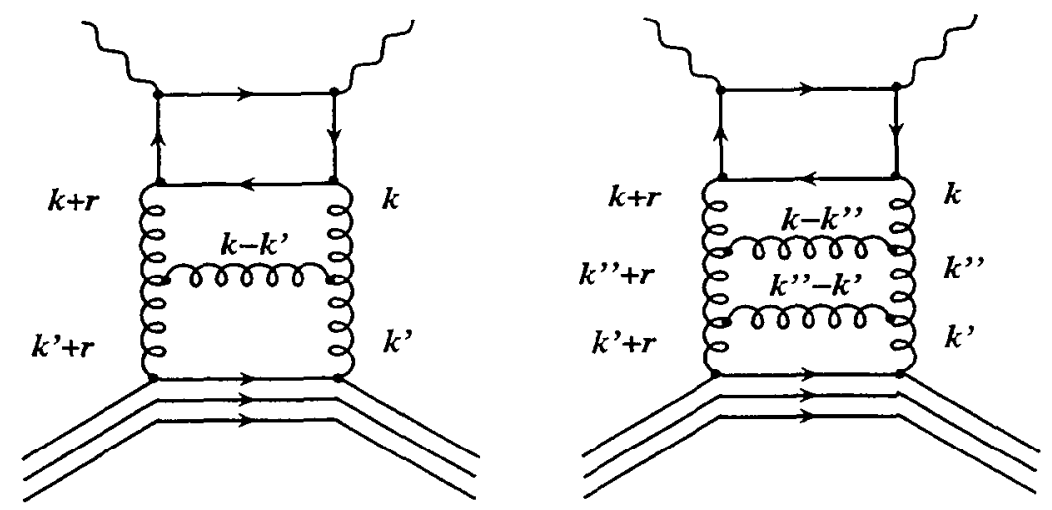

FIG. 2. Typical diagrams in the next-to-leading nd next-to-next-to-leading order in perturbation theory

where

$$
\begin{aligned}
v\left(x, Q^{2} / t\right)= & \left(\frac{1}{2} \ln ^{2} \frac{Q^{2}}{|t|}+2\right)+\frac{3 \alpha_{s}(Q)}{\pi} \ln \frac{1}{x}\left(\frac{1}{6} \ln ^{3} \frac{Q^{2}}{|t|}+2 \ln \frac{Q^{2}}{|t|}-2+\zeta(3)\right) \\
& +\frac{1}{2}\left(\frac{3 \alpha_{s}(Q)}{\pi} \ln \frac{1}{x}\right)^{2}\left(\frac{1}{24} \ln ^{4} \frac{Q^{2}}{|t|}+\ln ^{2} \frac{Q^{2}}{|t|}+2(\zeta(3)-1) \ln \frac{Q^{2}}{|t|}+1.46\right) \\
v^{\prime}\left(x, Q^{2} / t\right)= & 1+\frac{3 \alpha_{s}(Q)}{\pi} \ln \frac{1}{x}+\frac{1}{2}\left(\frac{3 \alpha_{s}(Q)}{\pi} \ln \frac{1}{x}\right)^{2} .
\end{aligned}
$$

In the numerical analysis we keep only the spin-independent part of the amplitude

$$
V_{\perp} \equiv \frac{1}{4} \sum e_{\perp}^{A} e_{\perp}^{B} V^{A B}=\frac{2}{x}\left(\frac{\alpha_{s}(Q)}{\pi}\right)^{2}\left(\sum_{\text {fiavors }} e_{q}^{2}\right) F_{1}^{p+n}(t) v\left(x, Q^{2}, t\right)
$$

(other contributions are numerically small).

The above expressions give us the imaginary part of the DVCS amplitude. For the calculation of the DVCS cross section we need to know also the real part of this amplitude which can be estimated via the dispersion relation:

$$
\begin{aligned}
& R \equiv \frac{1}{\pi} \operatorname{Re} H_{\perp}=\frac{2}{x}\left(\frac{\alpha_{s}}{\pi}\right)^{2}\left(\sum_{\text {flavors }} e_{q}^{2}\right)\left(F_{1}^{p}(t)+F_{1}^{n}(t)\right) r\left(x, Q^{2}, t\right) \\
& r\left(x, Q^{2}, t\right)=\frac{\pi}{2}\left[\frac{3 \alpha_{s}}{\pi}\left(\frac{1}{6} \ln ^{3} \frac{Q^{2}}{|t|}+2 \ln \frac{Q^{2}}{|t|}-2+\zeta(3)\right)+\right. \\
& \left.\left(\frac{3 \alpha_{s}}{\pi}\right)^{2} \ln \frac{1}{x}\left(\frac{1}{24} \ln ^{4} \frac{Q^{2}}{|t|}+\ln ^{2} \frac{Q^{2}}{|t|}+2(\zeta(3)-1) \ln \frac{Q^{2}}{|t|}+1.46\right)\right] .
\end{aligned}
$$

In order to estimate the cross section for DVCS at HERA kinematics $\left(Q^{2}>6 \mathrm{GeV}^{2}\right.$ and $\left.x<10^{-2}\right)$ we will use formulas from Ref. [6] with the trivial substitution $\frac{1}{2 x} F_{2}(x) R^{-1} e^{b t / 2} \rightarrow$ 
$V_{\perp}\left(x, Q^{2}, t\right)$. The expressions for the DVCS cross section, the QED Compton (Bethe-Heitler) cross section, and the interference term have the form $(\bar{y} \equiv 1-y)^{\dagger}$ :

$$
\begin{aligned}
& \frac{d \sigma^{\mathrm{DVCS}}}{d x d y d t d \phi_{r}}=\pi \alpha^{3} x \frac{1+\bar{y}^{2}}{Q^{4} y}\left(V_{\perp}^{2}\left(x, Q^{2}, t\right)+R_{\perp}^{2}\left(x, Q^{2}, t\right)\right) \\
& \frac{d \sigma^{\mathrm{QEDC}}}{d x d y d t d \phi_{r}}=\frac{\alpha^{3}}{\pi x} \frac{y\left(1+\bar{y}^{2}\right)}{|t| Q^{2} \bar{y}}\left(\left(F_{1}^{p}(t)\right)^{2}+\frac{|t|}{4 m^{2}}\left(F_{p}^{2}(t)\right)^{2}\right) \\
& \frac{d \sigma^{\mathrm{INT}}}{d x d y d t d \phi_{r}}=\mp 2 \alpha^{3} \frac{\left(1+\bar{y}^{2}\right)}{Q^{3} \sqrt{\bar{y}|t|}} R_{\perp}\left(x, Q^{2}, t\right) F_{1}^{p}(t) \cos \phi_{r} .
\end{aligned}
$$

Here $y=1-\frac{E^{\prime}}{E}\left(E\right.$ and $E^{\prime}$ are the incident and scattered electron energies, respectively, as defined in the proton rest frame) and $\phi_{r}=\phi_{e}+\phi_{N}$ where $\phi_{N}$ is the azimuthal angle between the plane defined by $\gamma^{*}$ and the final state proton and the $x-z$ plane and $\phi_{e}$ is the azimuthal angle between the plane defined by the initial and final state electron and $x-z$ plane (see Ref. [6]). We approximate the Dirac and Pauli form factors of the proton by the dipole formulas.

The asymmetry [6]

$$
A=\frac{\int_{-\pi / 2}^{\pi / 2} d \phi_{\tau} d \sigma^{\mathrm{DQI}}-\int_{\pi / 2}^{3 \pi / 2} d \phi_{\tau} d \sigma^{\mathrm{DQI}}}{\int_{0}^{2 \pi} d \phi_{r} d \sigma^{\mathrm{DQI}}}
$$

shows the relative importance of the interference term proportional to the real part of the DVCS amplitude $\left(d \sigma^{\mathrm{DQI}} \equiv d \sigma^{\mathrm{DVCS}}+d \sigma^{\mathrm{QEDC}}+d \sigma^{\mathrm{INT}}\right)$. The plots of asymmetry versus $y$ and $|t|$ are given by Fig. 3 .

The ratio of the DVCS and Bethe-Heitler cross sections [6]

$$
D(y, t) \equiv \frac{d \sigma_{D V C S}}{d \sigma_{Q E D C}}=\frac{4 \pi^{2}\left(\sum e_{q}^{2}\right)^{2}\left(v^{2}+r^{2}\right)\left(\frac{\alpha_{s}}{\pi}\right)^{4}\left(1+\frac{|t|}{4 m^{2}}\right) \bar{y} \frac{|t|}{Q^{2}}}{y^{2}\left(1+7.84 \frac{|t|}{4 m^{2}}\right)}
$$

is presented on Fig. 4. We see that there is a sharp dependence on $y$; at $y>0.2$ the DVCS part is negligible in comparison to Bethe-Heitler background whereas at $y<0.05$ the QEDC background is small in comparison to DVCS.

\footnotetext{
tThe expression for the interference term from ref. [6] is corrected by factor 2 [?], [11]
} 

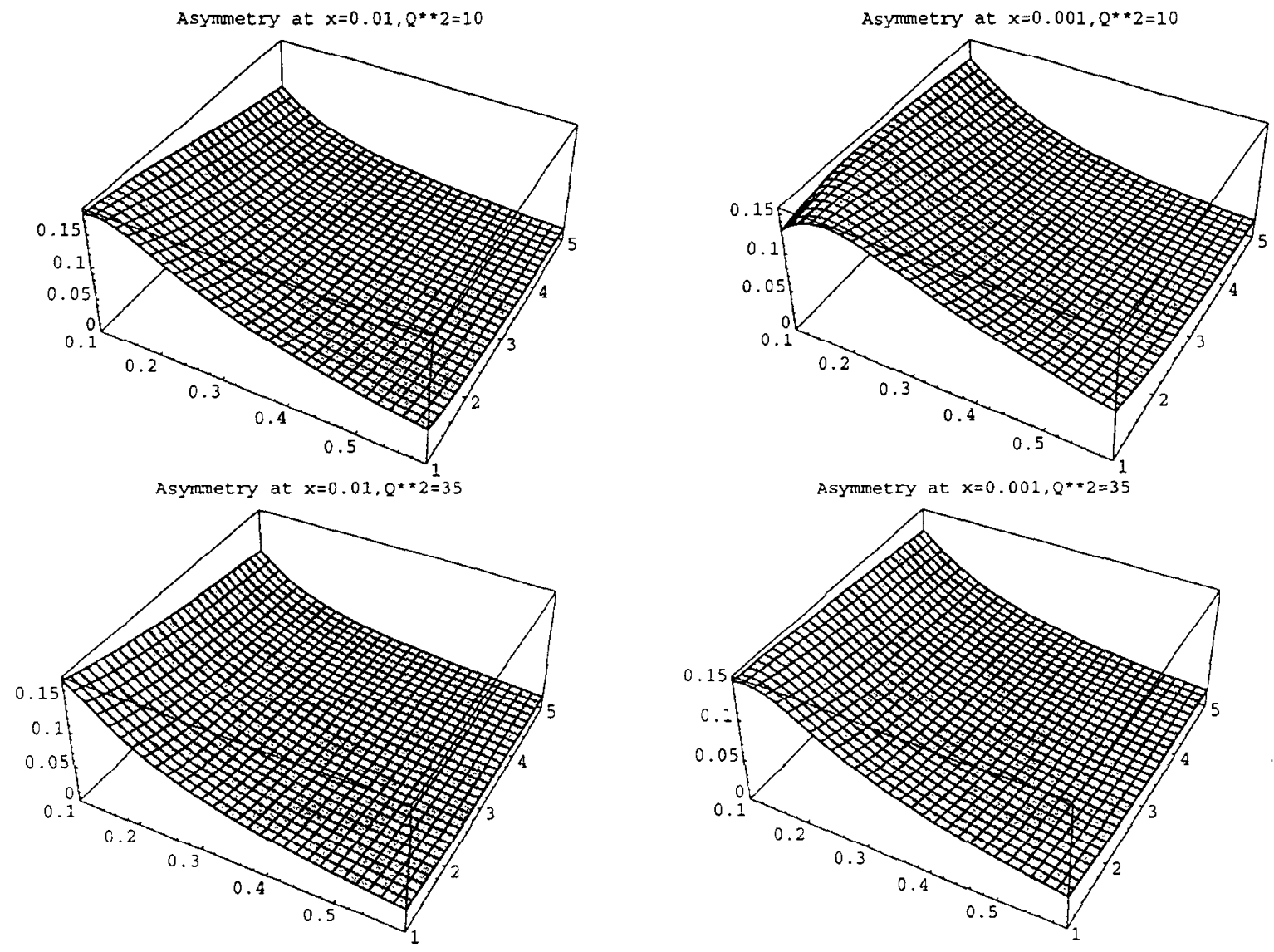

FIG. 3. Asymmetry versus $y=0.1 \div 0.6$ and $|t|=1 \div 5 \mathrm{GeV}^{2}$.

Finally let us estimate the relative weight of the DVCS signal (starting from $|t|=1$ $\mathrm{GeV}^{2}$ ) as compared to the DIS background. We define (cf. ref. [6])

$$
\begin{aligned}
& R_{\gamma}=\frac{\sigma\left(\gamma^{*}+p \rightarrow \gamma+p\right)}{\sigma\left(\gamma^{*}+p \rightarrow \gamma^{*}+p\right)} \simeq \\
& \frac{4 \pi \alpha}{Q^{2} F_{2}\left(x, Q^{2}\right)}\left(\frac{\alpha_{s}}{\pi}\right)^{4}\left(\sum c_{q}^{2}\right)^{2} \int_{1}^{Q^{2}} d t\left(F_{1}^{p+n}(t)\right)^{2}\left(v^{2}\left(x, Q^{2} / t\right)+r^{2}\left(x, Q^{2} / t\right)\right)
\end{aligned}
$$

At $Q^{2}=10 \mathrm{GeV}^{2}$ we find $R_{\gamma}=1.56 \times 10^{-5}$ for $x=0.01$ and $R_{\gamma}=2.36 \times 10^{-5}$ for $x=0.001$, while for $Q^{2}=35 \mathrm{GeV}^{2}$ we find $R_{\gamma}=0.62 \times 10^{-5}$ for $x=0.01$ and $R_{\gamma}=0.71 \times 10^{-5}$ for $x=0.001$.

The DVCS in the kinematical region (1) is probably the best place to test the momentum transfer dependence of the BFIL pomeron. The $t$-dependence of the BFKL pomeron 

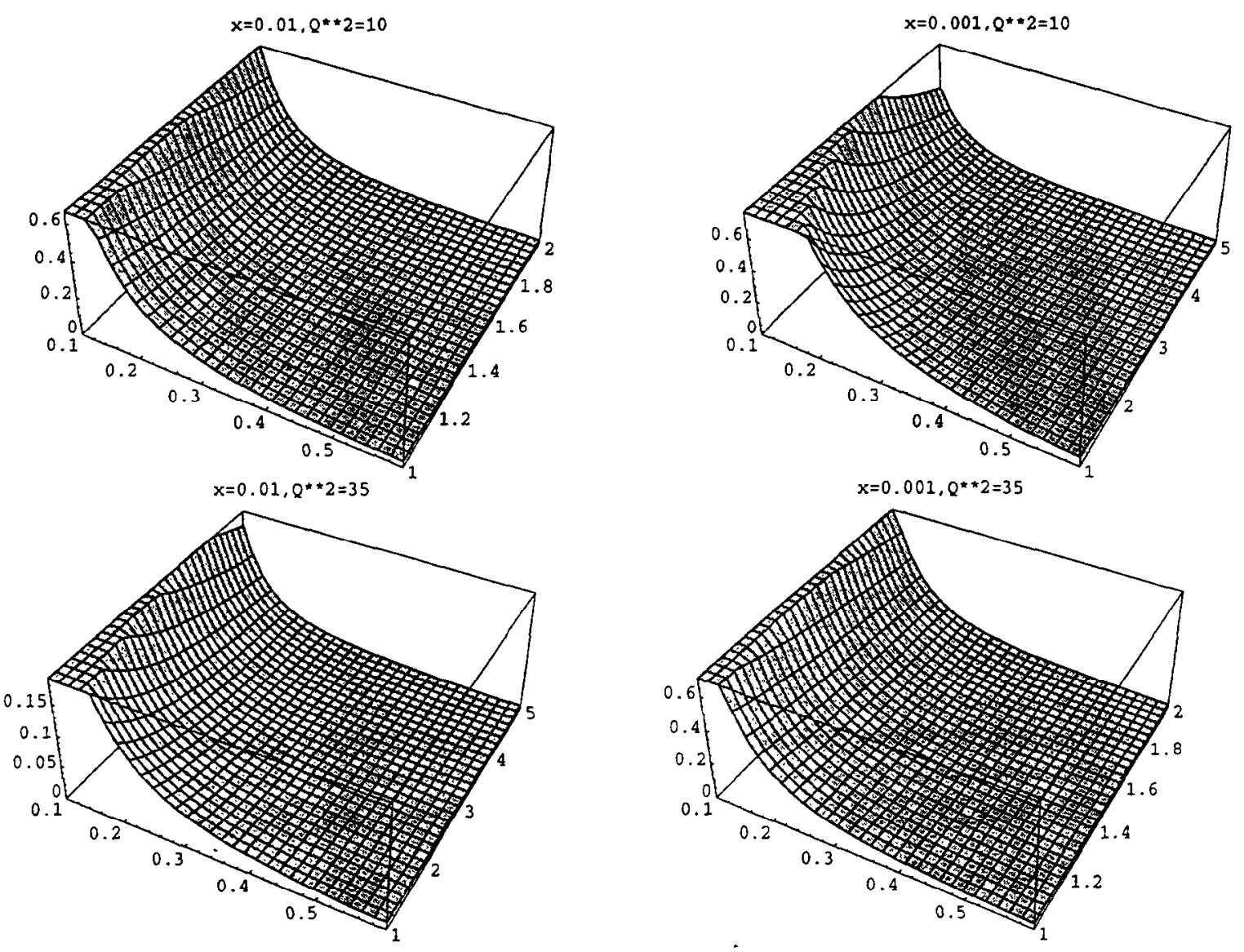

FIG. 4. The ratio $D\left(x, Q^{2} / t\right)$ versus $y=0.1 \div 0.6$ and $|t|=1 \div 5 \mathrm{GeV}^{2}$

changes the cross section at $t>2 \mathrm{GeV}^{2}$ by orders of magnitude and therefore it should be be possible to detect it. The pQCD calculation of the DVCS amplitude in the region (1) is in a sense even more reliable than the calculation of usual DIS amplitudes since it does not rely on the models for nucleon parton distributions. Indeed, all the non-perturbative nucleon input is contained in the Dirac form factor of the nucleon which is known to a pretty good accuracy.

Note that the spin dependence of our cross sections is trivial. This can be traced back to the fact that the spin-flip part of the skewed parton distributions is small at large $t$ if we use the dipole model for the form factors. It is known, however, that for the electric form factor the dipole model does not work well so there may be a non-trivial polarization effects 
even at large $t$.

\section{REFERENCES}

[1] D.Muller, D.Robaschik, B. Geyer, F.M. Dittes, and J. Horejsi, Fortschr.Phys. 42, 101 (1994)

[2] X. Ji, Phys. Lett. B 78, 610 (1997), Phys. Rev. D 55, 7114 (1997)

[3] A.V. Radyushkin,Phys. Lett. B 380, 417 (1996),Phys. Lett. B 385, 333 (1996)

[4] V.S. Fadin, E.A. Kuraev, and L.N. Lipatov, Phys. Lett. B 60, 50 (1975);

I.I. Balitsky and L.N. Lipatov, Sov. Journ. Nucl. Phys. 28, 822 (1978)

[5] [ZEUS Collaboration], Observation of DVCS in $e^{+}$Interactions at HERA, paper submitted to EPS HEP99 Conference, Tampere, 1999 (see also http://www-zeus.desy.de)

[6] L.L. Frankfurt, A. Freund, and M.I. Strikman, Phys. Rev. D 58, 114001 (1998), ErratumPhys. Rev. D 59, 119901 (1999)

[7] P.A.M. Guichon, M. Vanderhaeghen, Prog.Part.Nucl.Phys. 41, 125 (1998)

[8] A.V. Radyushkin, Phys. Rev. D 56, 5524 (1997)

[9] J.C. Collins, L.L. Frankfurt, and M.I. Strikman, Phys. Rev. D 56, 2982 (1997)

[10] I. Balitsky and E. Kuchina, hep-ph/0004059, Phys. Rev. D, in press.

[11] A.V. Belitsky, D. Mueller, L. Niedermeier, and A. Shaefer, hep-ph/0004059. 


\title{
P-A Physics at RHIC
}

\author{
Jen-Chieh Peng \\ Los Alamos National Laboratory, Los Alamos, NM 87545
}

The purpose of this talk is to address the question - can one study some of the eRHIC physics using the P-A collision at RHIC? I will first briefly summarize the highlights of a series of fixed-target experiments carried out at Fermilab measuring high-mass dimuons produced in P-A collisions. The prospect for extending dilepton production measurcments to high $\sqrt{s}$ and low $x$ at RHIC is discussed. Some preliminary results from a simulation study of the PHENIX detector at RHIC are also presented.

Figure 1 lists the physics topics studied in the E772/E789/E866 P-A dimuon production experiments at Fermilab [1]. One of the major physics goals of eRHIC is to study the saturation effects of parton distributions at small $x$ via the nuclear dependence measurements in e-A collisions. Extensive studies of nuclear dependence has been carried out in the Fermilab experiments both for the continuum Drell-Yan process and for the quarkonium production $\left(J / \Psi, \Psi^{\prime}, \Upsilon\right)$. It is clear that most of the physics topics listed in Figure 1 could be further pursued in P-A collision at RHIC.

The Drell-Yan process in P-A interaction is closely related to the Deep-Inelastic Scattering (DIS) in e-A interaction. A unique feature of proton-nucleus Drell-Yan process is that it probes the antiquark distributions in the target nucleus. This is complementary to the DIS which probes the sum of quark and antiquark distributions. Indeed, the Drell-Yan data have been used to determine the up and down sea quark distributions in the proton in various global parton distribution function parametrizations.

While logarithmic scaling violation is well established in DIS experiments, it is not well confirmed in DY experiments at all. No evidence for scaling violation is seen. As discussed in a recent review [1], there are mainly two reasons for this. First, unlike the DIS, the DY cross section is a convolution of two structure functions. Scaling violation implies that the structure functions rise for $x \leq 0.1$ and drop for $x \geq 0.1$ as $Q^{2}$ increases. For proton-induced DY, one often involves a beam quark with $x_{1}>0.1$ and a target antiquark with $x_{2}<0.1$. Hence the effects of scaling violation are partially cancelled. Second, unlike the DIS, the DY experiment can only probe relatively large $Q^{2}$, namely, $Q^{2}>16 \mathrm{GeV}^{2}$ for a mass cut of $4 \mathrm{GeV}$. This makes it more difficult to observe the logarithmic variation of the structure functions in DY experiments.

RHIC provides an interesting opportunity for unambiguously establishing scaling violation in the DY process [2]. Figure 2 shows the predictions for $p+d$ at $\sqrt{s}=100,200$, and 500 $\mathrm{GeV}$. The scaling-violation accounts for a factor of two drop in the DY cross sections when $\sqrt{s}$ is increased from $38.9 \mathrm{GeV}$ to $500 \mathrm{GeV}$. It appears quite feasible to establish scaling violation in $\mathrm{DI}^{*}$ with future dilepton production experiments at RHIC.

The flavor asymmetry between the $\bar{u}$ and $\bar{d}$ antiquark distributions in the proton has been clearly established by the E866 experiment [3] and the Hermes experiment [4]. RHIC offers the opportunity to measure $\bar{d} / \bar{u}$ asymmetry to very small $x$. Such information is important for an accurate determination of the integral of $\bar{d}-\bar{u}$. as well as for a better understanding of 
the origins for flavor asymmetry.

To make a more quantitative study for dilepton production in P-A collisions at RHIC, we have done some simulation for the PHENIX detector. The PHENIX detector consists of a forward and a backward muon arm for dimuon detection, as well as a barrel detector capable of detecting dielectrons. Figure 3 shows the expected Drell-Yan event distributions for a onemonth $p+d$ run of PHENIX. The forward and backward muon arms and the barrel detector cover different regions in $x_{F}, x_{1}$, and $x_{2}$. Only events with dilepton mass greater than $4 \mathrm{GeV}$ are considered in order to exclude the $J / \Psi$ and $\Psi^{\prime}$. The statistical accuracy for measuring $\sigma^{p d} / 2 \sigma^{p p}$ in a two-month PHENIX run is shown in Figure 4. Also shown in Figure 4 is the data from E866. The coverage in $x_{2}$ will be greatly extended at RHIC. In particular, the lowest $x_{2}$ reachable at $\mathrm{RHIC}$ is around $10^{-3}$, an order of magnitude lower than in E866.

An exciting feature at RHIC is that the detectors allow a measurement of other particles emitted in coincidence with the lepton pairs. In particular, it might be possible to measure "diffractive" Drell-Yan process in which Drell-Yan dileptons are accompanied by an energetic neutron or proton carrying a large fraction of the incident proton beam momentum. Such a process is the hadronic analog of the diffractive DIS process observed recently at HERA [5]. Assuming factorization, one could write down the following expression for "diffractive" DrellYan cross section:

$$
d \sigma^{D Y} / d y d m d x_{F}\left(p+p \rightarrow N+\mu^{+} \mu^{-}+x\right)=d \sigma^{D Y} / d m d x_{F}\left(p+M \rightarrow \mu^{+} \mu^{-}+x\right) \cdot f_{M B}(y),
$$

where $y$ is the fraction of the proton beam momentum carried by the baryon (neutron or proton) detected at forward angles. $f_{M B}(y)$ is the probablity for proton to fluctuate into a meson-baryon pair where the baryon now carries a fraction $y$ of the initial proton momentum. The underlying picture of the diffractive Drell-Yan process is as follows. A proton fluctuates into a nucleon plus a meson ( $p \rightarrow p+\pi^{\circ}, p \rightarrow n+\pi^{+}$, for example) and the meson subsequently interacts with the other proton beam producing a lepton pair. The remaining baryon moves roughly along the initial proton beam direction and could be detected in a forward-angle detector. The cross section is therefore a convolution of the probability function $f_{M B}(y)$ and the proton-meson Drell-Yan cross section. Figure 5 shows $f_{M B}$ for $N \rightarrow \pi N$ and $N \rightarrow \pi \Delta$ using the formulation in Ref. [6]. A simulation for the $p+p \rightarrow n+\mu^{+} \mu^{-}+X$ for the PHENIX detector, where the muon pairs are detected in the muon arms and the neutrons are detected by a small-angle calorimeter (such as the Zero Degree Calorimeter), has been carried out and the result is shown in Figure 6.

If diffractive Drell-Yan process can be measured at RHIC, it will provide an independent determination of $f_{M B}(y)$. This will allow a direct test of the meson-cloud model which can successfully explain the $\bar{d} / \bar{u}$ asymmetry of the nucleon sea [3]. One could also tag on forwardgoing $\Delta$ or $\Lambda$. The $\Lambda$-tagging is of special interest since it can shed light on the strange-quark contents of the proton. Another extension is the measurement of double-helicity asymmetry, $A_{L L}$, in diffractive $\vec{p}+\vec{p}$ Drell-Yan process. Since the underlying process involves proton-pion interaction, $A_{L L}$ is expected to be zero. Double diffractive Drell-Yan process, which involves pion-pion interaction, can also be contemplated. Finally, it is natural to extend diffractive Drell-Yan measurement to diffractive $J / \Psi$ production.

A suppression of the Drell-Yan yields from heavy nuclear targets is observed in E772/E866 at small $x_{2}[\bar{l}]$. This is consistent with the shadowing effect observed in DIS. In fact, E772/E866 provide the only experimental evidence for antiquark shadowing. The reach of 
small $x_{2}$ in E772/E866 is limited by the mass cut $(M \geq 4 \mathrm{GeV})$ and by the relatively small $\sqrt{s}$. P-A collisions at RHIC clearly offer the exciting opportunity to extend the study of shadowing to smaller $x$. Figure 7 compares the E772 $\mathrm{Fe} / \mathrm{D}$ Drell-Yan ratio data with what could be obtained at PHENIX in a two-month run. The coverage in $x_{2}$ will be significantly increased not only for small- $x_{2}$ but also for the large- $x_{2}$ region.

In contrast to the Drell-Yan process, large nuclear effects are found in the hadronic production of $J / \Psi, \Psi^{\prime}$, and $\Upsilon$. A detailed study of the $J / \Psi$ and $\Psi^{\prime}$ A-dependence as a function of $p_{T}$ and $x_{F}$ was recently reported by E866 [8]. P-A collisions at RHIC would offer two unique opportunities for further clarifying the mechanisms for nuclear effects in quarkonium production. First, RHIC can reach much smaller values of $x_{2}$, as shown in Figure 8. Second, the negative $x_{F}$ region, which could not be easily covered by fixed-target experiments, can be well studied at RHIC as shown in Figure 9.

\section{References}

[1] P. L. McGaughey, J. M. Moss and J. C. Peng, Annu. Rev. Nucl. Part. Sci. 19 (1999) 217 (hep-ph/9905409).

[2] J. C. Peng, AIP Conference Proceedings, 494 (1999) 503 (hep-ph/9912371).

[3] E. A. Hawker et al., Phys. Rev. Lett. 80 (1998) 3715; J. C. Peng et al., Phys Rev. D58 (1998) 092004.

[4] K. Ackerstaff et al., Phys. Rev. Lett. 81 (1998) 5519.

[5] C. Adloff et al., Eur. Phys. J. C6 (1999) 587.

[6] S. Kumano, Phys. Rev. D43 (1991) 3067.

[7] D. A. Alde et al., Phys. Rev. Lett. 64 (1990) 2479; M. A. Vasiliev et al., Phys Rev. Lett. 83 (1999) 2304.

[8] M. J. Leitch et al., Phys. Rev. Lett. 84 (2000) 3256 (nucl-ex/9909007). 
Physics Topics Studied at the FNAL Dimuon Experiments

1. Nuclear Effects of Dimuon Production

- Enhancement of Antiquark Contents in Nuclei?

- Partonic Energy Loss in Nuclei?

- Shadowing of Antiquarks in Nuclei?

2. Nuclear Effects of Quarkonium Production

- $J / \Psi, \Psi^{\prime}$, and $\Upsilon$ A-Dependence

- $x_{F}$ and $p_{T}$ Dependence for $J / \Psi$ and $\psi^{\prime}$

- Open Charm A-Dependence

3. Flavor Asymmetry of the Nucleon Sea

- $x$-Dependence of the $\bar{d} / \bar{u}$ Asymmetry

- Origins of the Nucleon Sea

4. Dilepton Production Mechanisms

- Drell-Yan Absolute Cross Section and Scaling

- Polarization of Drell-Yan and $J / \Psi$

- Large $p_{T}$ Production Mechanism

Figure 1: List of physics topics studied in the Fermilab dimuon experiments E772/E789/E866. 
$\mathrm{pA} \rightarrow \mu^{+} \mu^{-} \mathrm{X}$

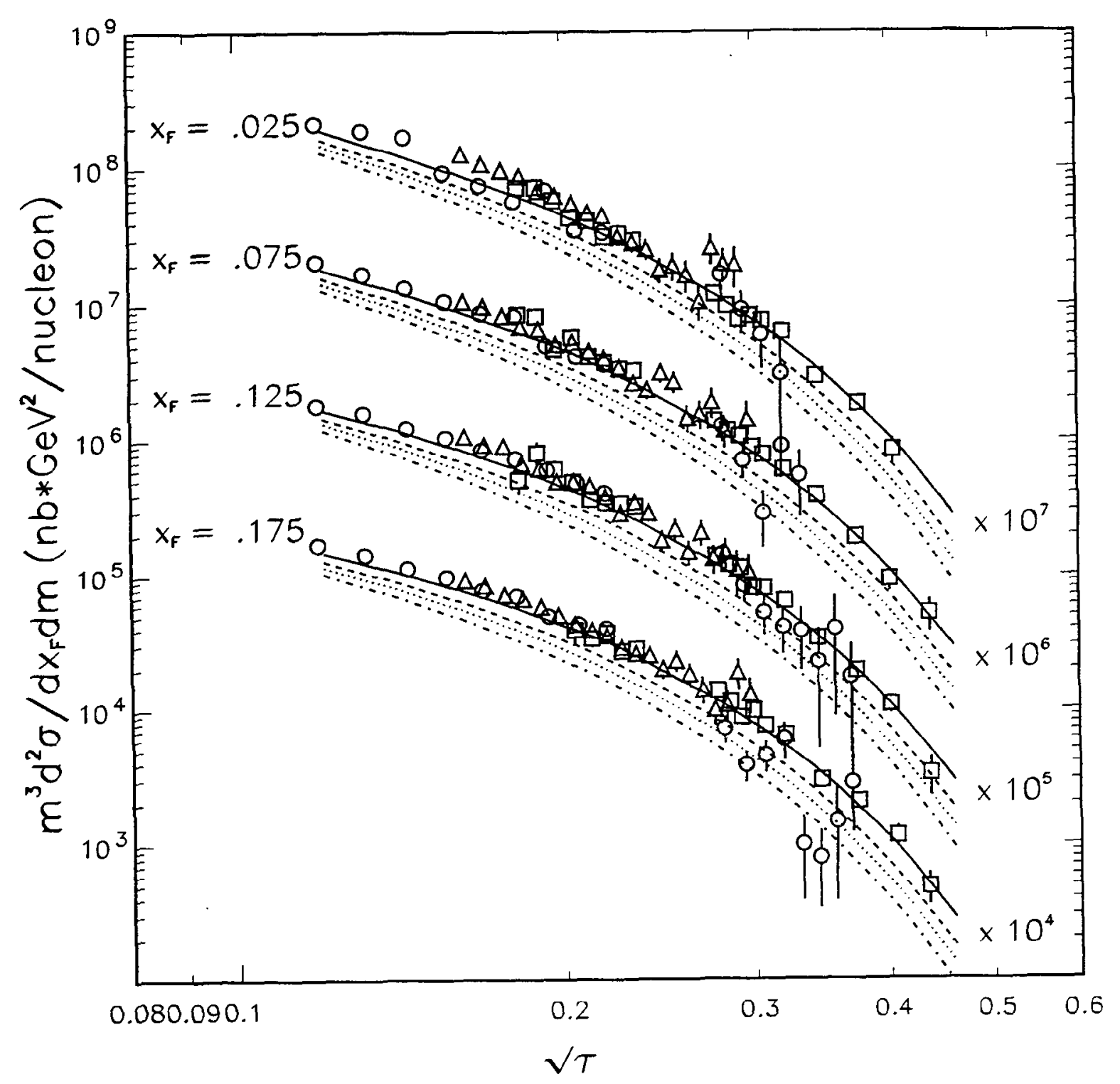

Figure 2: Comparison of DY cross sections with NLO calculations using MRST structure functions. The E772, E605, and NA3 data points are shown as circles, squares, and triangles, respectively. The solid curves correspond to fixed-target $\mathrm{p}+\mathrm{d}$ collision at $800 \mathrm{GeV}$, while the dashed, dotted, and dash-dotted curves correspond to $\mathrm{p}+\mathrm{d}$ collision at $\sqrt{s}=100,200$. 500 $\mathrm{GeV}$, respectively. 

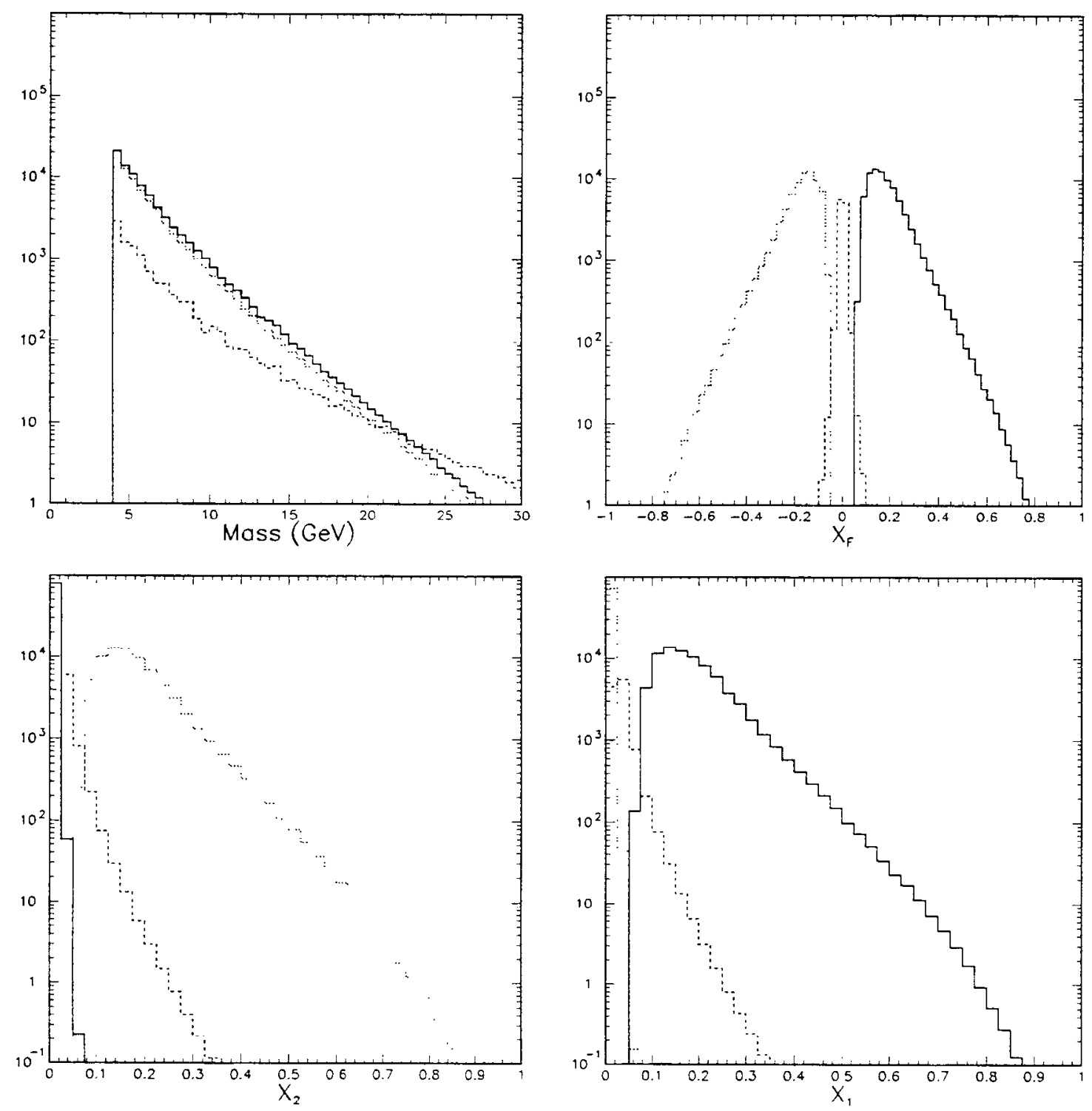

Figure 3: Expected $l^{+} l^{-}$DY event distributions for a one-month PHENIX run at $\sqrt{s}=$ $200 \mathrm{GeV}$. The solid and dotted curves correspond to dimuons detected in the forward and backward muon arms, respectivcly. The dashed curves correspond to $e^{+} e^{-}$events detected in the PHENIX barrel detector. 


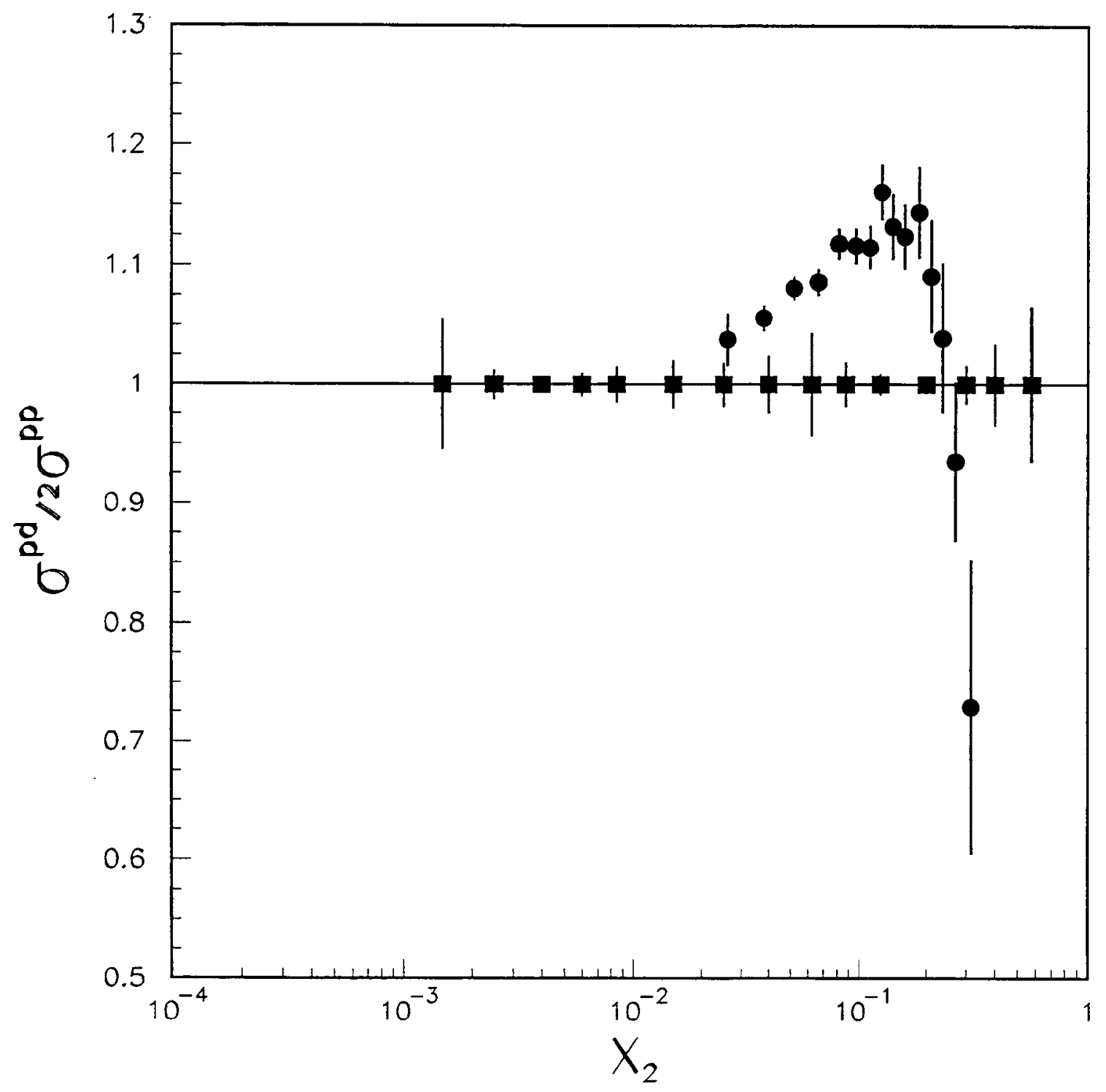

Figure 4: The solid circles are the $\sigma^{p d} / 2 \sigma^{p p}$ data from E866. The solid squares show the kinematic coverage in $x_{2}$ and the expected statistical accuracy for a two-month $\mathrm{p}+\mathrm{p}$ plus $\mathrm{p}+\mathrm{d}$ run. Both the dimuon and dielectron detections have been taken into account. 


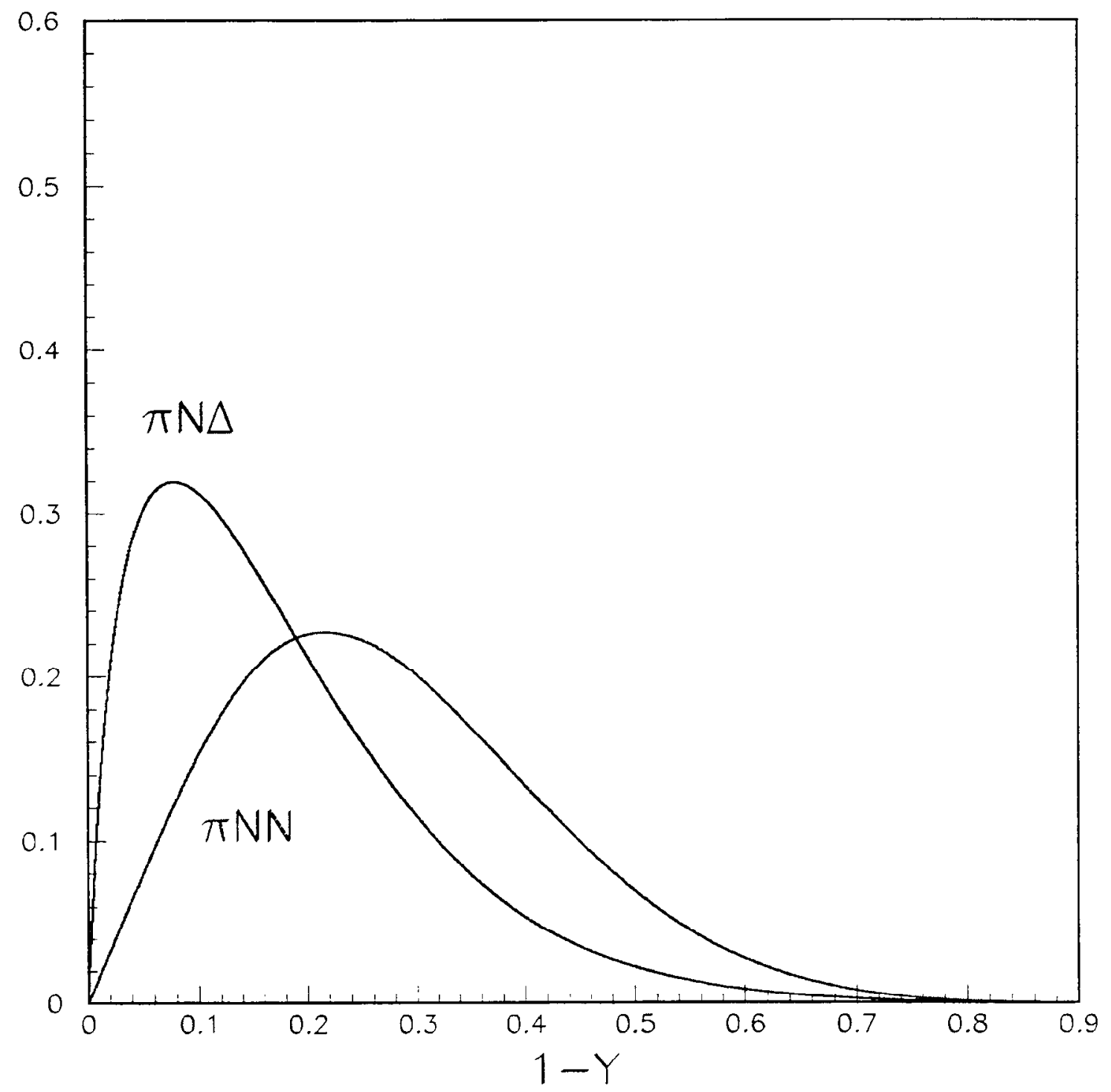

Figure 5: Probability distributions for $N \rightarrow N \pi$ and $N \rightarrow \Delta \pi$ according to the model of Ref. [6]. $Y$ is the fraction of the nucleon momentum carried by the baryon $(N$ or $\Delta)$ and $1-Y$ is the fractional momentum carried by the pion. 


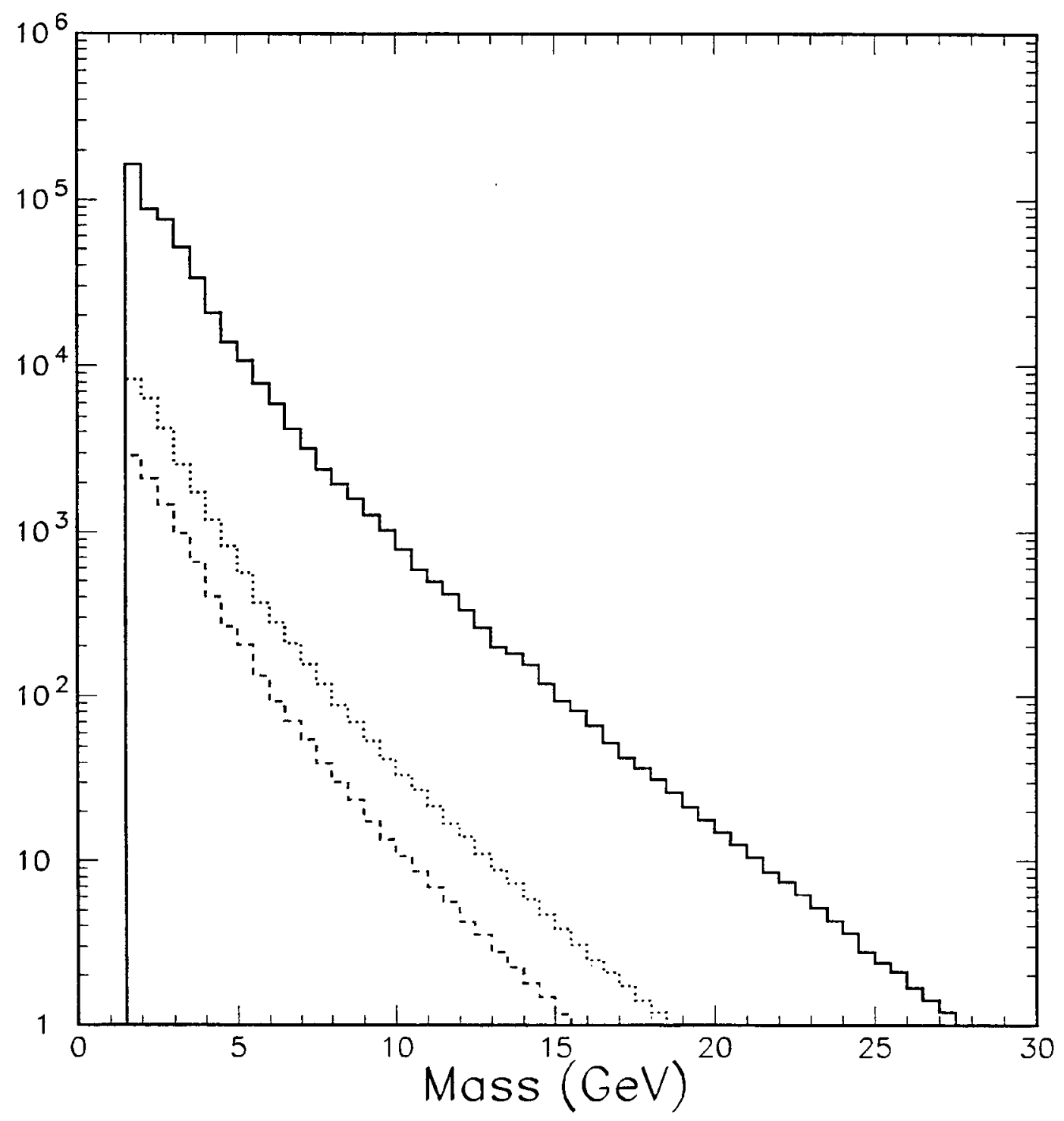

Figure 6: The solid curve corresponds to dimuon event distributions in one muon arm for a one-month $p+p$ PHENIX run at $\sqrt{s}=200 \mathrm{GeV}$. The dotted and dashed curves correspond to the yields for $p+p \rightarrow n+\mu^{+} \mu^{-}+X$ with $Y>0.6$ and $Y>0.8$, respectively, where $Y$ is the fraction of proton beam momentum carried by the neutron. 


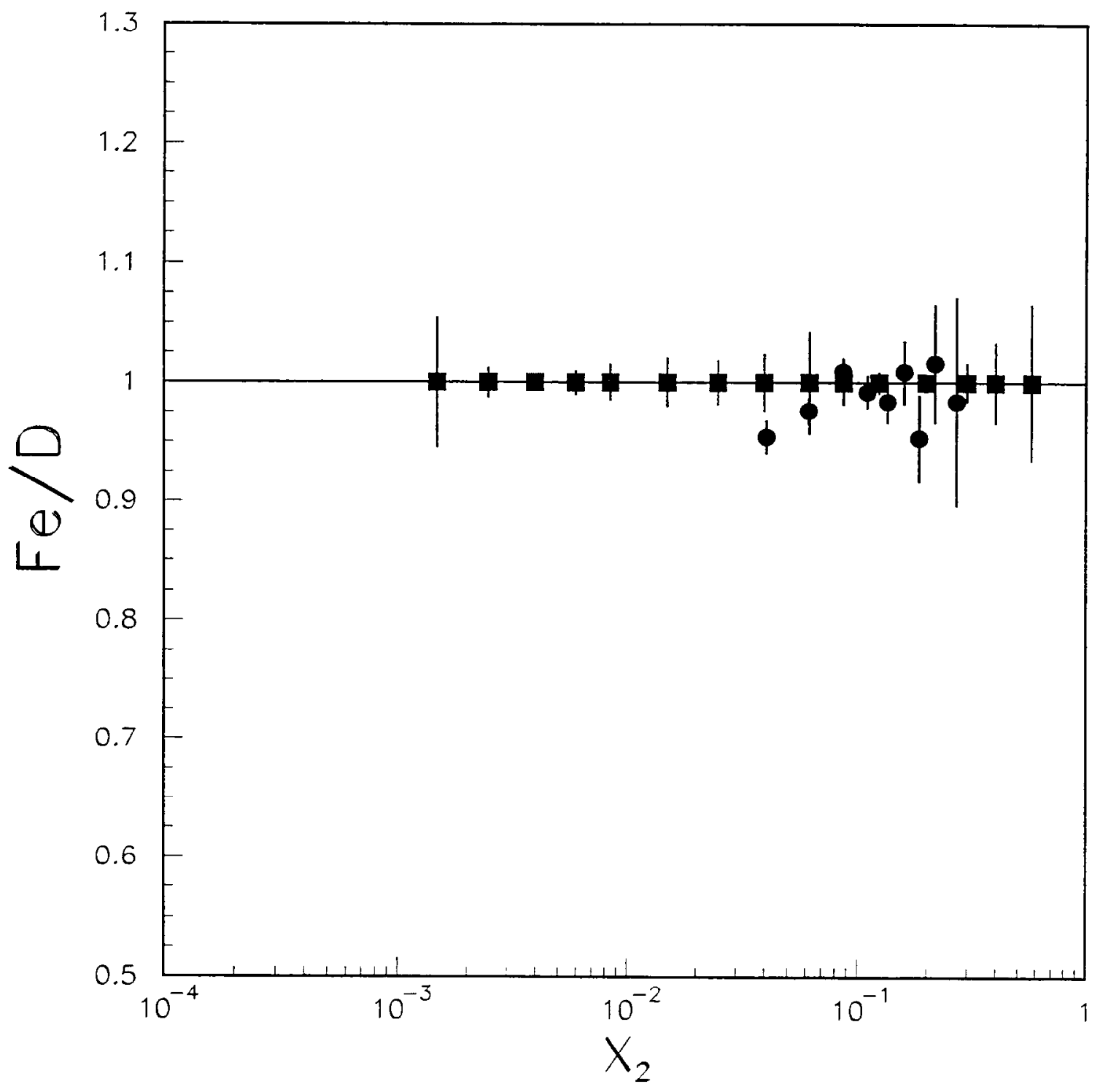

Figure 7: The solid circles are the $\sigma(p+F e) / \sigma(p+D)$ Drell-Yan cross section ratios from E772. The solid squares show the kinematic coverage in $x_{2}$ and the expected statistical accuracy for a two-month $\mathrm{p}+\mathrm{Fe}$ and $\mathrm{p}+\mathrm{D}$ run. Both the dimuon and dielectron detections have been taken into account. 


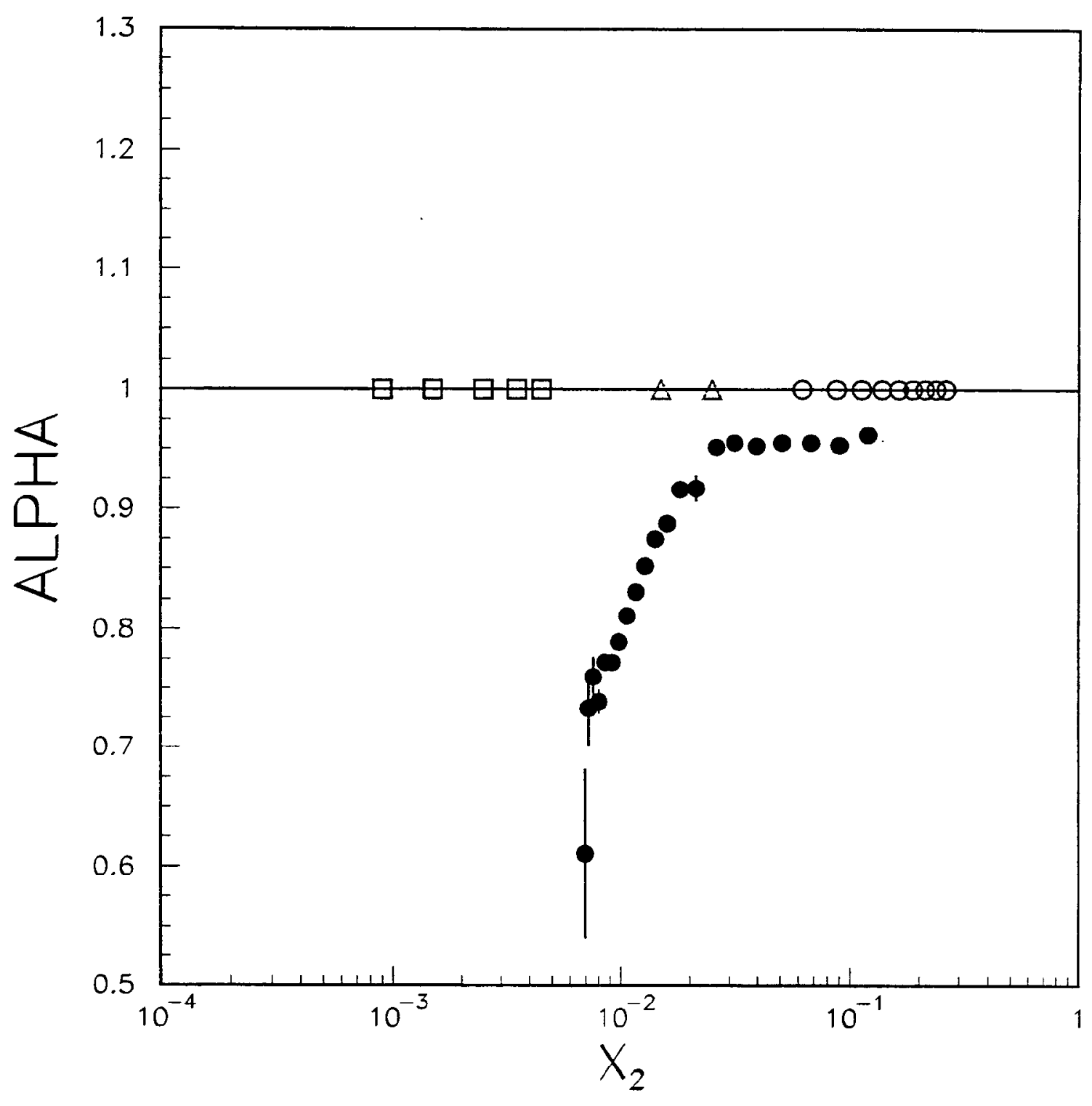

Figure 8: The solid circles are the $\alpha$ values $\left(\sigma=\sigma_{0} A^{\alpha}\right.$, where $\mathrm{A}$ is the mass number of the nucleus) obtained from the $J / \Psi$ A-dependence measurement of E866. The open squares, triangles, and circles correspond to the kinematic coverage in $x_{2}$ and the expected statistical accuracy for the forward muon arm, barrel detector, and backward muon arm, respectively, in a two-month $\mathrm{p}+\mathrm{p}$ plus $\mathrm{p}+\mathrm{A}$ run. 


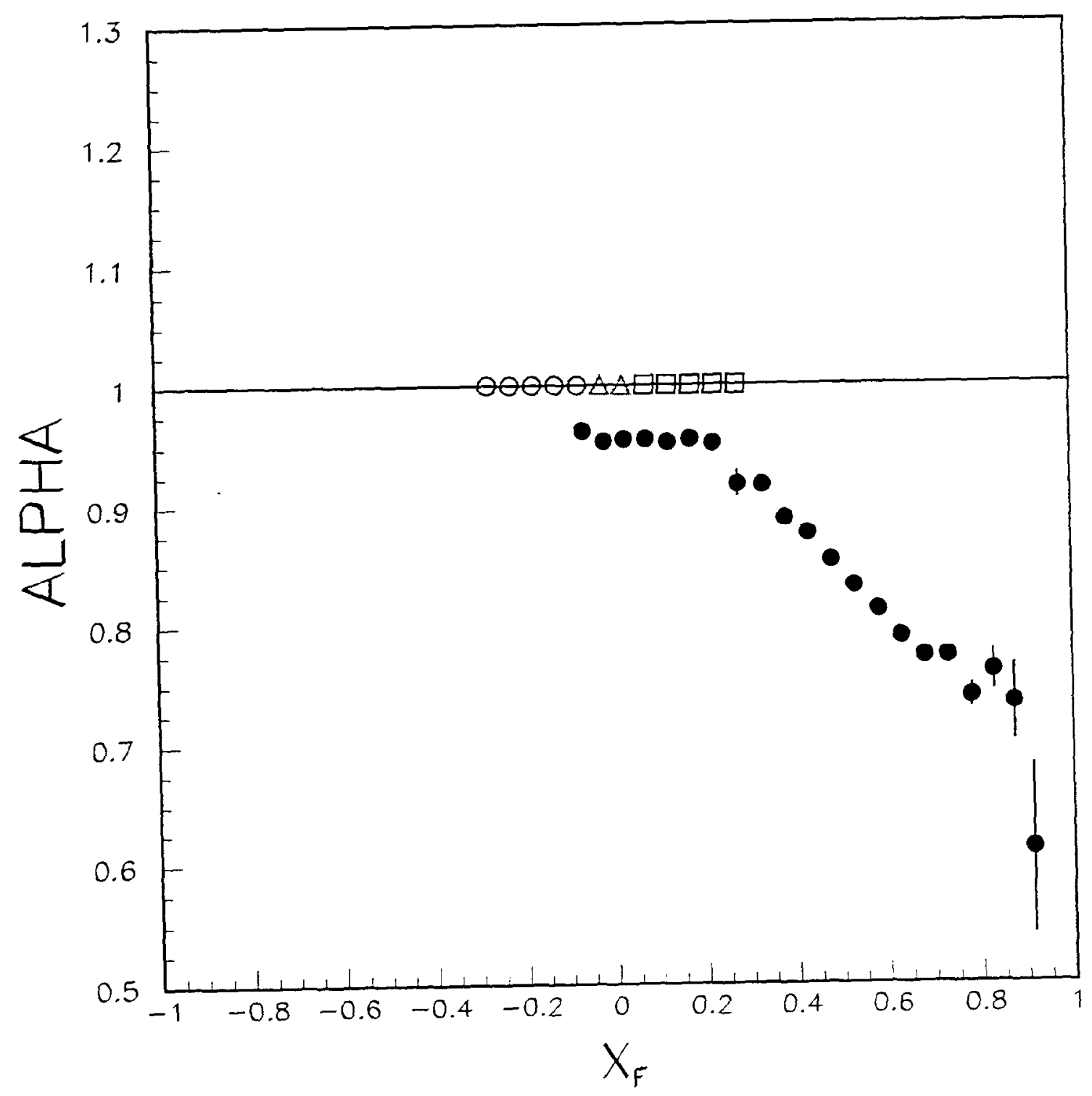

Figure 9: Same as Figure 8, but plotted as a function of $x_{F}$. 


\title{
Physics Issues at eRHIC
}

\author{
Yuri V. Kovchegov \\ Physics Department, Brookhaven National Laboratory, Upton, NY 11973
}

\section{MAPPING THE "PHASE DIAGRAM" OF HIGH ENERGY QCD}

One of the most fundamental problems in the physics of strong interactions is the problem of understanding QCD at high energies. The physics of high energy QCD is relevant to many scattering processes, such as proton-proton collisions (pp), deep inelastic scattering on a proton or nucleus (ep and eA), nuclear collisions (AA). The energies of the modern AA accelerators are beginning to reach up to hundreds of $\mathrm{GeV}$ per nucleon at Relativistic Heavy Ion Collider (RHIC) at BNL and at Large Hadron Collider (LHC) which is being constructed at CERN. To be able to properly interpret the data to be provided by these machines it is vital to clarify the underlying dynamics of strong interactions. It is also very important to understand that QCD dynamics at high energies leads to creation of very strong gluonic fields in the nuclear and hadronic wave functions. These strong fields are freed in the nuclear collisions producing gluonic matter with the field strength $F_{\mu \nu} \sim 1 / g$ at the early stages of the collisions. Subsequent interactions may equilibrate this gluonic matter, possibly leading to the state commonly known quark-gluon plasma (QGP). However, since the maximum possible in QCD gluonic field strength has been reached at the very early stages of the collision these final state interactions can not make it stronger. That way the strong gluonic fields will be created independent of the possible subsequent QGP formation. Final state rescatterings can only modify the distribution of particles in this gluonic matter, without changing the field strength. Thus it is very interesting and important to study the properties of this gluonic matter, which could be observed already in the wave function of a single nucleus. The proposed electron-heavy ion collider (eRHIC) is the machine which is perfectly suitable to explore the strong field physics.

The internal structure of hadrons and nuclei are usually described in terms of the quark and gluon distribution functions, $x q\left(x, Q^{2}\right)$ and $x G\left(x, Q^{2}\right)$. However, the observable quantities are the cross sections of DIS, which, in turn, are related to the structure functions $F_{1}\left(x, Q^{2}\right)$ and $F_{2}\left(x, Q^{2}\right)$. Proton's structure functions have been extensively studied at Hadron Electron Ring Accelerator (HERA) at DESY. The majority of the data shows that the proton's structure functions at large $Q^{2}$ and not very small $x$ can be described by the DGLAP evolution equation, which is a linear equation. It is convenient to present different properties of hadronic and nuclear structure functions in terms of a "phase diagram" in $Q^{2}$ and $\ln 1 / x$ plane (see Fig. 1). The DGLAP physics corresponds to the lower right section of the plane, where $Q^{2}$ is large and $x$ is not too small. As one goes towards smaller $x$ in the same region of $Q^{2}$ the hadronic and nuclear structure functions rise. Most of the data in that region can be explained in terms of either small- $x$ limit of DGLAP equation or, alternatively and more interestingly, by the BFKL equation, which is also a linear equation but could be responsible for evolving the system towards a higher gluonic density regime. However, the DIS cross sections can not rise forever. This, for instance, would violate unitarity. That means that at some very small $x$ the hadronic and nuclear structure function have to undergo a significant qualitative change of behavior becoming a much slower varying functions of $x$. The slow down of the growth of hadronic structure functions is usually associated with non-linear effects in the quark and gluon dynamics, such as parton recombination, which eventually balances the parton splitting process. Therefore the partons in the hadronic or nuclear wave function reach the state of saturation. The region of saturation of the structure functions is represented in yellow in Fig. 1. The Bjorken $x$ corresponding to the transition to the saturation region is different for different values of $Q^{2}$, increasing with decreasing $Q^{2}$. There has recently been obtained new experimental data at HERA which might contain evidence of saturation transition in DIS on a proton at $x \approx 10^{-3}-10^{-1}$ and $Q^{2} \approx 2-4 G e V^{2}$. There are, however, different possible explanations of the data. 


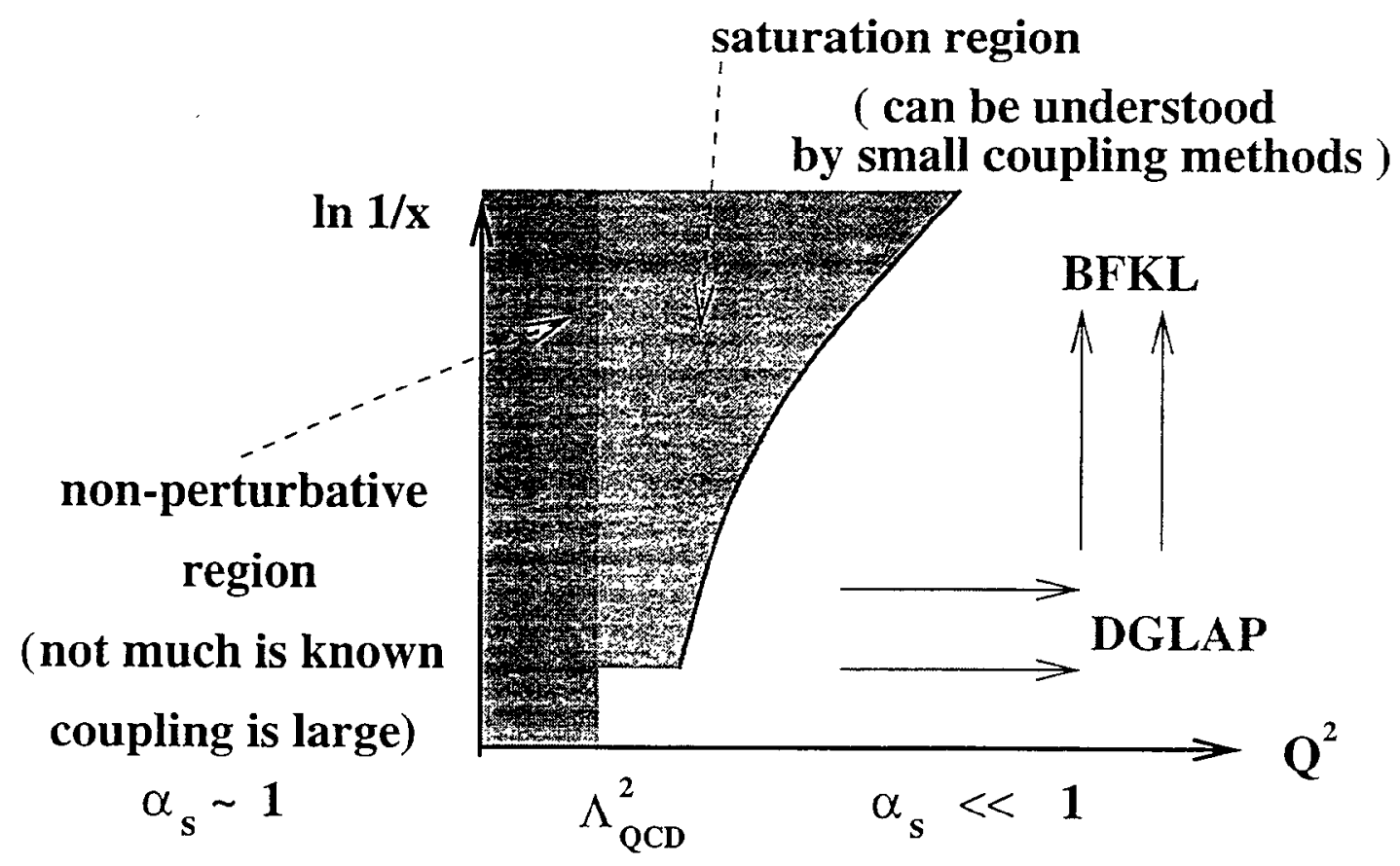

FIG. 1. "Phase diagram" of high energy QCD.

The saturation transition line varies as one goes from DIS on a proton to DIS on a nucleus. Roughly we can say that all the momenta in the system scale by powers of the atomic number $A$. The saturation transition scale, $Q_{s}^{2}(x)$, changes as $Q_{s}^{2}(x) \rightarrow A^{1 / 3} Q_{s}^{2}(x)$. That means that the whole saturation curve in Fig. 1 would be shifted towards higher momenta (to the right in Fig. 1) by a factor of say 5 or 6 for a heavy nucleus. The effect of saturation in DIS on a heavy ion would be much more pronounced, and will be accessible at much larger values of $x$ than in DIS on the proton. That means one needs much lower energies in eA colliders as compared to ep colliders to achieve the same density of partons. DIS on a heavy ion would allow us to observe saturation and the non-lincar effects of high gluonic density associated with it in the region of large $Q^{2}$, which would also allow the use of perturbation theory in the theoretical calculations, i. e. theoretical understanding of the process would be much more feasible than in ep collisions.

\section{EVOLUTION EQUATION DESCRIBING THE SATURATION REGION}

A non-linear evolution equation describing the nuclear $F_{2}$ structure function can be written in the large $N_{c}$ limit using Mueller's dipole model. The $F_{2}$ structure function can be written as a convolution of the wave function of the virtual photon splitting in the quark-antiquark pair $\Phi\left(\mathrm{x}_{01}, z\right)$ and the cross section (or forward amplitude) of the $q \bar{q}$ pair interacting with the target nucleus $N\left(\mathbf{x}_{01}, \mathbf{b}_{0}, Y\right)$ :

$$
F_{2}\left(x, Q^{2}\right)=\frac{Q^{2}}{4 \pi^{2} \alpha_{E M}} \int \frac{d^{2} \mathbf{x}_{01} d z}{2 \pi} \Phi\left(\mathbf{x}_{01}, z\right) d^{2} b_{0} N\left(\mathbf{x}_{01}, \mathbf{b}_{0}, Y\right) .
$$

The forward amplitude $N\left(\mathrm{x}_{01}, \mathrm{~b}_{0}, Y\right)$ obeys the following evolution equation [i]

$$
\begin{gathered}
N\left(\mathbf{x}_{01}, \mathbf{b}_{0}, Y\right)=-\gamma\left(\mathbf{x}_{01}, \mathbf{b}_{0}\right) \exp \left[-\frac{4 \alpha C_{F}}{\pi} \ln \left(\frac{x_{01}}{\rho}\right) Y\right]+\frac{\alpha C_{F}}{\pi^{2}} \int_{0}^{Y} d y \exp \left[-\frac{4 \alpha C_{F}}{\pi} \ln \left(\frac{x_{01}}{\rho}\right)(Y-y)\right] \\
\times \int_{0} d^{2} x_{2} \frac{x_{01}^{2}}{x_{02}^{2} x_{12}^{2}}\left[2 N\left(\mathbf{x}_{02}, \mathbf{b}_{0}+\frac{1}{2} \mathbf{x}_{12}, y\right)-N\left(\mathbf{x}_{02}, \mathbf{b}_{0}+\frac{1}{2} \mathbf{x}_{12}, y\right) N\left(\mathbf{x}_{12}, \mathbf{b}_{0}+\frac{1}{2} \mathbf{x}_{02}, y\right)\right],
\end{gathered}
$$


with $\gamma\left(\mathbf{x}_{01}, \mathbf{b}_{0}\right)$ the propagator of a dipole of size $\mathbf{x}_{01}$ at the impact parameter $\mathbf{b}_{0}$ through the target nucleus or hadron, which was taken to be of Glauber form

$$
\gamma\left(\mathbf{x}_{01}, \mathbf{b}_{0}\right)=\exp \left[-\frac{\alpha \pi^{2}}{2 N_{c} S_{\perp}} \mathrm{x}_{01}^{2} A x G\left(x, 1 / \mathbf{x}_{01}^{2}\right)\right]-1 .
$$

here $S_{\perp}$ is the transverse area of the hadron or nucleus, $A$ is the atomic number of the nucleus, and $x G$ is the gluon distribution of the nucleons in the nucleus, which was taken at the two gluon (lowest in $\alpha$ ) level. $Y$ is the rapidity variable. Eq. (3) is written for a cylindrical hadron or nucleus. In the large $N_{c}$ limit $C_{F} \approx N_{c} / 2$. In Eq. (2) $\rho$ is an ultraviolet regulator.

Eq. (2) describes the QCD evolution including multiple BFKL pomeron exchanges. In terms of diagrams it corresponds to summation of the "fan" diagrams of Fig. 2.

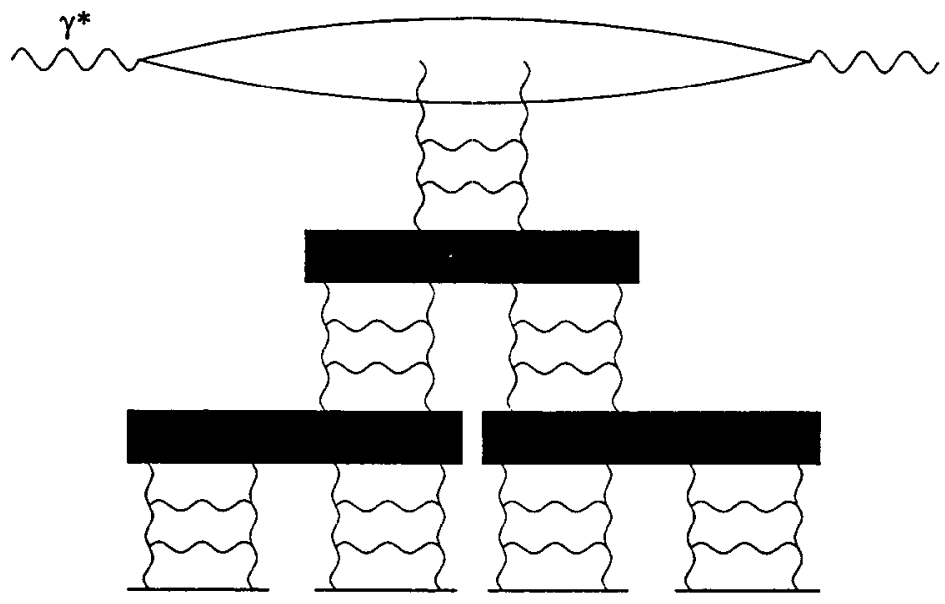

FIG. 2. An example of the pomeron "fan" diagram.

The solution of Eq. (2) for the case of a nuclear target yields us with the amplitude $N$ which, for not very large values of rapidity behaves like a single zero momentum transfer BFKL pomeron exchange [2]

$$
N\left(x_{\perp}, Y\right)=C_{-1} \Lambda x_{\perp} \frac{\exp \left[\left(\alpha_{P}-1\right) Y\right]}{\sqrt{14 \alpha N_{c} \zeta(3) Y}} \exp \left(-\frac{\pi}{14 \alpha N_{c} \zeta(3) Y} \ln ^{2} x_{\perp} \Lambda\right)
$$

and saturates to a constant as energy gets very high $[2]$

$$
N\left(x_{\perp}, Y\right) \approx 1, \quad Y \geq \frac{1}{\alpha_{P}-1} \ln \frac{1}{\alpha^{2}} .
$$

The qualitative plot of $N$ as a function of energy is shown in Fig. 3.

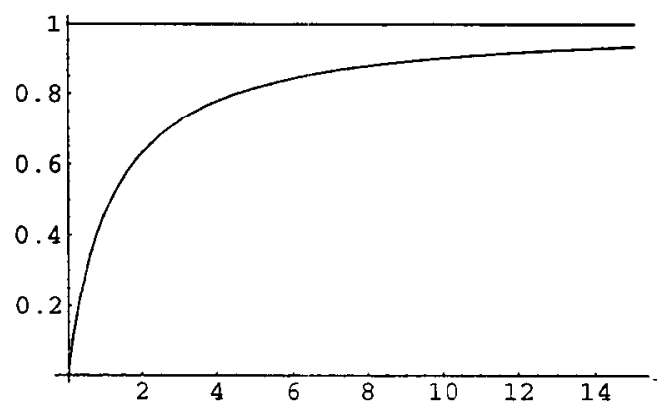

FIG. 3. Plot of the qualitative behavior of the forward $q \bar{g}$ pair scattering amplitude on a nucleus, $N\left(x_{\perp}, Y\right)$, as a function of $x_{\perp} Q_{s}(Y)$ (one BFKL pomeron exchange contribution). At small values of $x_{\perp} Q_{s}$ the structure function is linear in $x_{\perp} Q_{s}$, reproducing BFKL pomeron. As energy increases the forward amplitude $N$ saturates to a constant. 
One can see that Eq. (2) describes the transition into the saturation region.

The corresponding high energy asymptotics for $F_{2}$ is given by [2]

$$
F_{2}\left(x, Q^{2}\right) \approx \frac{Q^{2} R^{2} N_{c}}{3 \pi^{2}}\left(\alpha_{P}-1\right) Y, \quad Y \geq \frac{1}{\alpha_{P}-1} \ln \frac{1}{\alpha^{2}}
$$

Thus $F_{2}$ continues growing with energy, but the growth is much slower than the power of energy growth associated with a single pomeron exchange. Eq. (6) also does not violate unitarity bound.

The saturation scale $Q_{s}$ predicted by Eq. 2 is [2]

$$
Q_{s}(Y)=\Lambda \frac{C_{-1}}{r 4 \ln 2} \frac{\exp \left[\left(\alpha_{P}-1\right) Y\right]}{\sqrt{14 \alpha N_{c} \zeta(3) Y}}
$$

where $r$ is a constant and $C_{-1}$ is given by the initial conditions. Note that since $C_{-1}$ scales as $A^{1 / 3}$ with the nuclear atomic number the saturation scale generated by BFKL-like dynamics scales as $Q_{s}^{2} \sim A^{2 / 3}$, which gives a much faster growth with $A$ than the usual Glauber-type estimates of previous section. Thus at the eRHIC collider the slow down of the growth of $F_{2}^{A}$ with energy could be measured at not extremely small $x$ and at somewhat high $Q^{2}$. If observed, the effect would signify the discovery of saturation phenomena.

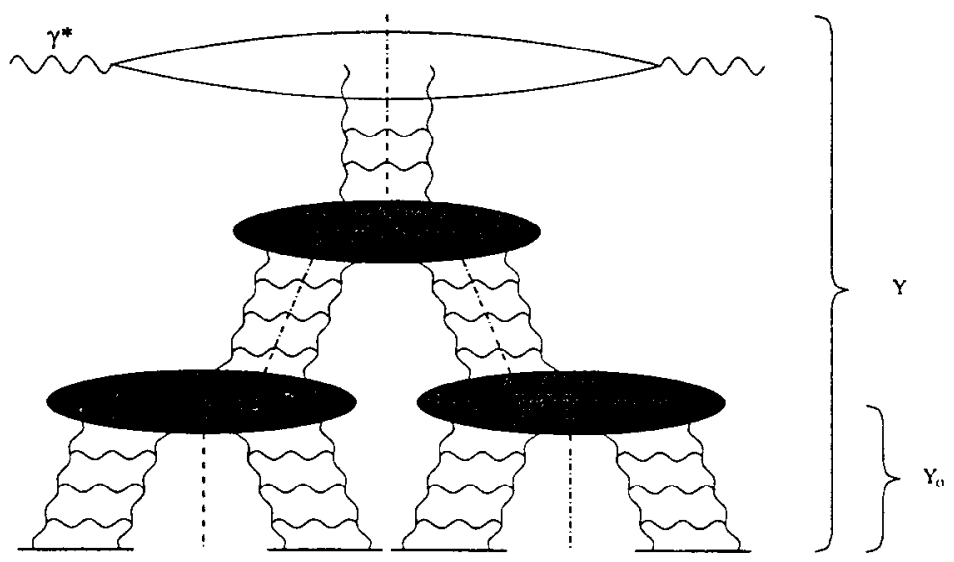

FIG. 4. A diagram contributing to the diffractive dissociation with rapidity gap $Y_{0}$ as considered in the text.

\section{SINGLE DIFFRACTIVE DISSOCIATION}

Let us consider single diffractive dissociation in DIS on a nucleus that leaves the nucleus intact producing a rapidity gap adjacent to the nucleus in the final state. The process is depicted in Fig. 4. Similarly to the above we can express the structure function for single diffractive dissociation as a convolution of virtual photon's wave function and the cross section of the single diffractive dissociation of the quark-antiquark pair on a nucleus

$$
F_{2}^{S D}\left(x, Q^{2}, Y_{0}\right)=\frac{Q^{2}}{4 \pi^{2} \alpha_{E M}} \int \frac{d^{2} \mathbf{x}_{01} d z}{4 \pi} \Phi\left(\mathbf{x}_{01}, z\right) d^{2} b N^{D}\left(\mathbf{x}_{01}, \mathbf{b}, Y, Y_{0}\right)
$$

$N^{D}\left(\mathbf{x}_{01}, \mathbf{b}, Y, Y_{0}\right)$ includes all events with rapidity gap being equal to or greater that $Y_{0}$. For the quarkantiquark pair diffractive cross section we can write down the following evolution equation [3]

$$
\begin{gathered}
N^{D}\left(\mathbf{x}_{01}, \mathbf{b}, Y, Y_{0}\right)=N_{0}^{2}\left(\mathbf{x}_{01}, \mathbf{b}, Y_{0}\right) e^{-\frac{4 \alpha C_{F}}{\pi} \ln \left(\frac{x_{01}}{\mu}\right)\left(Y-Y_{0}\right)}+\frac{\alpha C_{F}}{\pi^{2}} \int_{Y_{0}^{\prime}}^{Y} d y e^{-\frac{4 \alpha C_{F}}{\pi} \ln \left(\frac{x_{02}}{p}\right)(Y-y)} \\
\times \int_{\rho} d^{2} x_{2} \frac{x_{01}^{2}}{x_{02}^{2} x_{12}^{2}}\left[2 N^{D}\left(\mathbf{x}_{02}, \mathbf{b}+\frac{1}{2} \mathbf{x}_{12}, y, Y_{0}\right)+N^{D}\left(\mathbf{x}_{02}, \mathbf{b}+\frac{1}{2} \mathbf{x}_{12}, y, Y_{0}\right) N^{D}\left(\mathbf{x}_{12}, \mathbf{b}+\frac{1}{2} \mathbf{x}_{02}, y, Y_{0}\right)\right.
\end{gathered}
$$




$$
\left.-4 N^{D}\left(\mathbf{x}_{02}, \mathrm{~b}+\frac{1}{2} \mathbf{x}_{12}, y, Y_{0}\right) N_{0}\left(\mathbf{x}_{12}, \mathbf{b}+\frac{1}{2} \mathbf{x}_{02}, y\right)+2 N_{0}\left(\mathbf{x}_{02}, \mathrm{~b}+\frac{1}{2} \mathbf{x}_{12}, y\right) N_{0}\left(\mathbf{x}_{12}, \mathbf{b}+\frac{1}{2} \mathbf{x}_{02}, y\right)\right] .
$$

Here $N_{0}$ is $N$ from Eq. (2) above. Eq. (9) resums multiple pomeron exchange contributions to diffractive dissociation shown in Fig. 4.

Define the quark-antiquark single diffractive cross section with a fixed rapidity gap $Y_{0}$

$$
R\left(\mathbf{x}_{01}, \mathrm{~b}, Y, Y_{0}\right)=-\frac{\partial N^{D}\left(\mathrm{x}_{01}, \mathrm{~b}, Y, Y_{0}\right)}{\partial Y_{0}} .
$$

Eq. (9) can be solved in a simple approximation. We can plot the resulting expression for $R$ as a function of rapidity gap $Y_{0}$ for fixed total rapidity interval $Y$ (see Fig. 5).

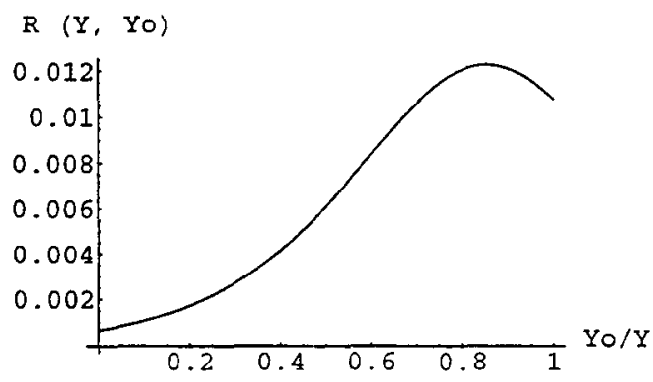

FIG. 5. The cross section of diffractive dissociation in units of $\pi R^{2}$ at fixed $Y$ as a function of $Y_{0}$, which is scaled by $Y$. The elastic contribution is not included.

As one can see from Fig. 5 the single diffractive cross section has a maximum at certain large rapidity interval. This effect is present only if one takes into account saturation (multiple pomeron exchanges) and can not be mimicked by the usual triple pomeron description of diffraction. It, therefore, can serve as an independent test of the onset of saturation.

[1] Yu. V. Kovchegov, Phys. Rev. D 60, 034008 (1999).

[2] Yu. V. Kovchegov, Phys. Rev. D 61, 074018 (2000).

[3] Yu. V. Kovchegov, E. Levin, Nucl. Phys. B577, 221 (2000). 


\title{
Gluon shadowing: perturbative or not?
}

\author{
Jamal Jalilian-Marian \\ Physics Department, University of Arizona, Tucson, AZ, USA
}

\begin{abstract}
We numerically compare the predictions of different $\mathrm{pQCD}$ based models for nuclear shadowing of gluons. We show that including initial non-perturbative shadowing effects enhances the amount of shadowing generated by these models.
\end{abstract}

\section{Introduction}

Understanding the modifications of gluon distribution function in a nuclear environment is both theoretically challenging and experimentally useful. It will be extremely important to have reliable predictions for the nuclear gluon distribution functions since these are the basic ingredients of any perturbative calculations involving gluons in the initial state. The knowledge of nuclear gluon distribution functions is required in order to understand the outcome of nuclear collisions. The experiments at RHIC and LHC will be sensitive to the modifications of the gluon distribution function in a nuclei.

The theoretical models of nuclear shadowing at small $x$ can be divided into two broad categories: non-perturbative and perturbative. The non-perturbative approach uses the Gribov-Regge language of soft pomerons and AGK cutting rules while the perturbative models are based on QCD. Within PQCD approaches, nuclear shadowing can be understood in the two different but complementary frames; the nuclear rest frame and the infinite momentum frame.

In the rest frame of a nucleus, nuclear shadowing is due to destructive interference between multiple scattering amplitudes between the virtual photon and different nucleons in the nucleus. Glauber-Mueller multiple scattering scheme is used to describe the scattering of a gluon pair on a nucleus where the scatterings are eikonal.

In the infinite momentum frame, nuclear shadowing is due to fusion of gluon ladders due to high parton multiplicity in a Lorentz contracted nucleus. In this picture, the usual linear evolution equation of DGLAP is modified due to higher twist high parton density effects. Such an evolution equation which resums all the high parton density effects was derived in [4]. This new equation was used in [5] to investigate nuclear shadowing of the gluon distribution function. In this talk, we report on the effects of initial non-perturbative shadowing on the $x$ and $Q^{2}$. dependence of the perturbative shadowing. 


\section{The nuclear gluon distribution function}

The nuclear gluon distribution function is defined via the shadowing ratio defined as

$$
S=\frac{x G_{A}}{A x G_{N}}
$$

where $x G_{A}$ and $x G_{N}$ are the nuclear and nucleon gluon distribution functions respectively. We will use the following evolution equation for gluons derived in [4]

$$
\frac{\partial^{2}}{\partial y \partial \xi} x G(x, Q)=\frac{3}{\pi^{3}} \pi R^{2} Q^{2} \exp \left(\frac{1}{\bar{\kappa}}\right) E_{1}\left(\frac{1}{\bar{\kappa}}\right)
$$

where $\bar{\kappa}$ is

$$
\bar{\kappa}=\frac{N_{c} \alpha_{s}}{\pi} \frac{\pi^{3}}{3} \frac{1}{\pi R^{2} Q^{2}} x G(x, Q) .
$$

This equation was solved numerically in [5] where we refer the reader for more details. Originally, it was assumed that at some initial point $x_{0}$ and $Q_{0}$, there is no shadowing and the shadowing ratio as defined by (1) is 1 . Motivated by experimental results on shadowing of structure functions $F_{2}$, the initial point $x_{0}, Q_{0}$ was taken to be $x_{0} \sim 0.05-0.06$ and $Q_{0} \sim 1 \mathrm{GeV}$. The equation was then numerically evolved to the point $x, Q$ using a 4 th order Runge-kutta algorithm. The result is shown in Fig. 1:

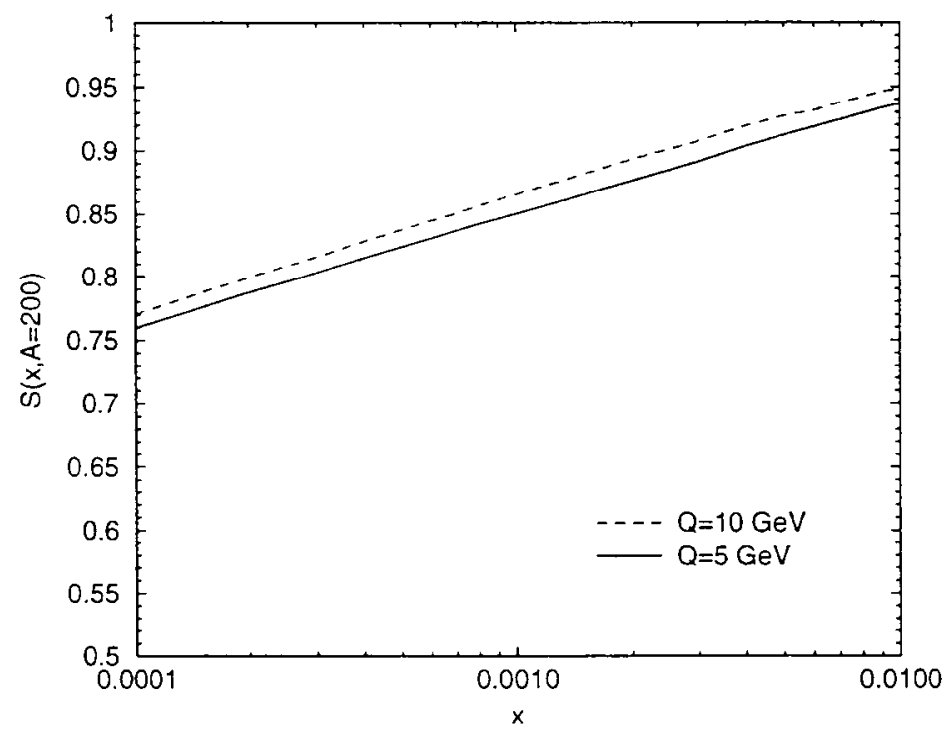

Figure 1: shadowing as a function of $x$ at $Q=5 \mathrm{GeV}$ and $Q=10 \mathrm{GeV}$.

We also show the shadowing predicted by another model, base on Glauber multiple scattering, [6] which uses the following evolution equation for gluon shadowing:

$$
\frac{\partial^{2}}{\partial y \partial \xi} x G^{A}(x, Q)=\frac{3}{4 \pi^{2}} R_{A}^{2} Q^{2}\left[C+\ln \left(\kappa_{a g l}\right)+E_{1}\left(\kappa_{a g l}\right)\right]
$$


where $C \sim 0.57$ is the Euler constant and

$$
\kappa_{a g l}=4 \frac{N_{c} \alpha_{s}}{\pi} \frac{\pi^{3}}{3} \frac{1}{\pi R^{2} Q^{2}} x G^{A}\left(x, Q^{2}\right)
$$

We use the same exact approximations in solving this equation as above. The gluon shadowing predicted by the different approaches is shown in Fig. 2 where $S^{G L R}$ refers to the shadowing ratio predicted if one kept only the first non-linear term in the expansion of equations $(2,4)$ :

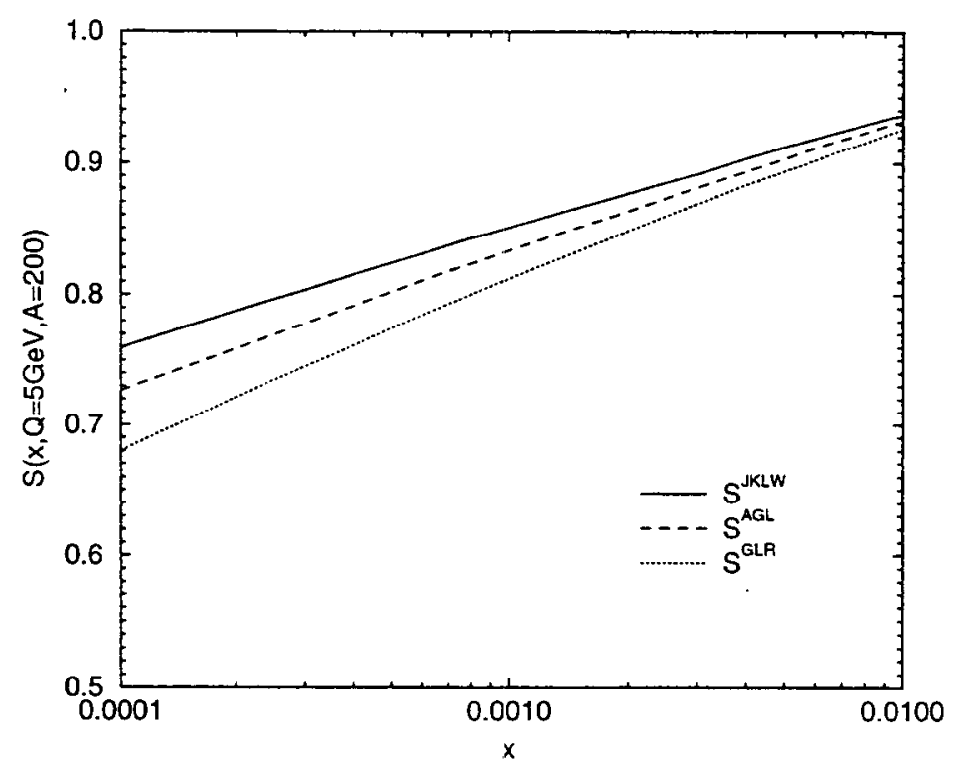

Figure 2: Model dependence of shadowing as a function of $x$ at $Q=5 \mathrm{GeV}$.

Finally, we show the effect of initial non-perturbative shadowing on the perturbative evolution in Fig. 3. Here we use a known parameterization of shadowed nuclear gluon distribution function [7]. This clcarly shows that the initial shadowing effects are important and can not be neglected, at least for the RHIC and LHC experiments. As one goes to smaller and smaller $x$, it is expected that the initial shadowing will become less and less important. This is verified theoretically in calculations based on Glauber multiple scattering formalism [8]. However, the most important issue is perhaps the $Q$ dependence of the gluon shadowing which as shown here is independent of the initial shadowing since the parameterization of initial gluon shadowing in [7] is independent of $Q$. Since the initial non-perturbative shadowing is believed to be $Q^{2}$ independent, any measured variation of shadowing ration with $Q^{2}$ will be an indication of perturbative shadowing. This is an important issue which can be studied in a wide kinematic region in DIS experiments on nuclei such as eRHIC. 


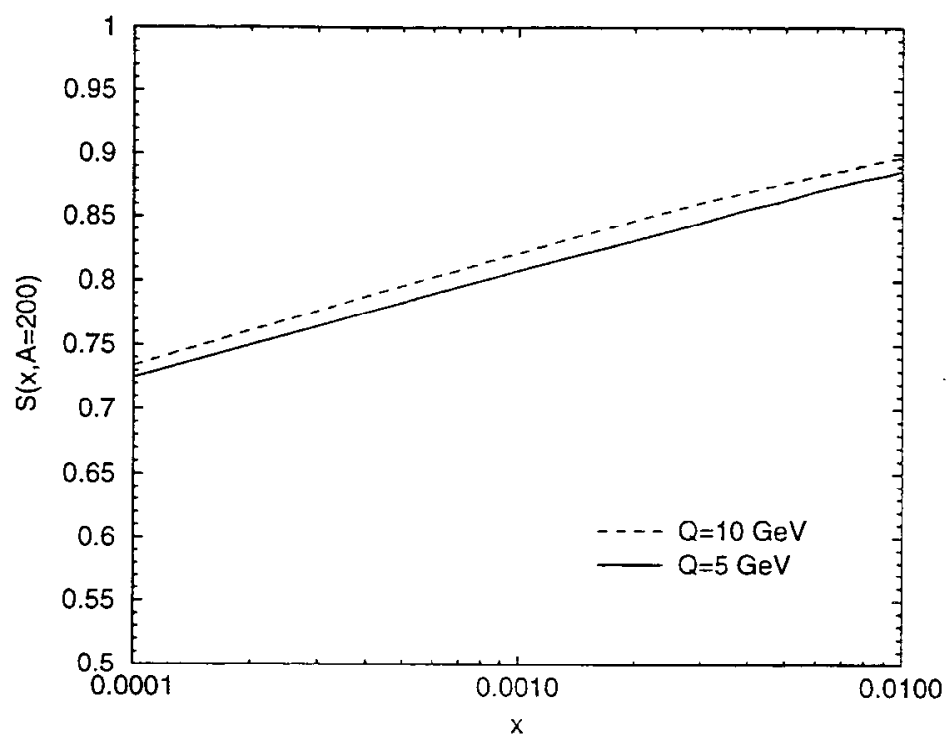

Figure 3: Shadowing as a function of $x$ at $Q=5 \mathrm{GeV}$ and $Q=10 \mathrm{GeV}$ including initial shadowing.

\section{Acknowledgments}

I would like to thank the organizers of the workshop and all of my collaborators and specially Xin-Nian Wang with whom much of the numerical work presented here has been done.

\section{References}

[1] M. Arneodo, Phys. Rep. 240, 301 (1994).

[2] M.R. Adams, et.al., Phys. Rev. Lett. 68, 3266 (1992).

[3] L. McLerran and R. Venugopalan, Phys. Rev. D49, 335 (1994); D49, 2233 (1994); A. Ayala, J. Jalilian-Marian, L. McLerran and R. Venugopalan, Phys. Rev. D52, 2935 (1995); D53, 458 (1996).

[4] J. Jalilian-Marian, A. Kovner, L. McLerran and H. Weigert, Phys. Rev. D55, 5414 (1997); J. Jalilian-Marian, A. Kovner, A. Leonidov and H. Weigert, Nucl. Phys. B504, 415 (1997); Phys. Rev. D59, 014014 (1999); D59, 014015 (1999);

D59, 034007 (1999); A. Kovner and J.G. Milhano. hep-ph/9904420.

[5] J. Jalilian-Marian and X.N. Wang. Phys. Rev. D60, 054016 (1999), hep$\mathrm{ph} / 0005071$.

[6] A.L. Ayala, M.B Gay Ducati and E.M Levin, Nucl. Phys. B493, 305 (1997); B511, 355 (1998).

[7] C.J. Benesh, J. Qiu and J.P. Vary. Phys. Rev. C50. 1015 (1994). 
[8] Z. Huang, H.J. Lu and I. Sarcevic, Nucl. Phys. A637, 79 (1998). 


\title{
Computing the Gluon Structure Functions of Nucleons and Nuclei
}

\author{
Dmitri Kharzeev ${ }^{1,2}$ \\ 1 Physics Department, Brookhaven National Laboratory \\ Upton, NY 11973, USA \\ ${ }^{2}$ RIKEN-BNL Research Center, Brookhaven National Laboratory \\ Upton, NY 11973, USA
}

In this talk, based on the joint work with Yuri Kovchegov and Eugene Levin [1], we show how the scale anomaly matching can be used to evaluate the initial condition for the DGLAP evolution equation. We also estimate higher twist contributions to the gluon structure functions of the nucleon and heavy nuclei.

The cross section of a DIS process can be written down as a dispersion relation with respect to the masses $M$ and $M^{\prime}[2]$

$$
\sigma(j+p)=\int \frac{<0|j| M>d M^{2}}{Q^{2}+M^{2}} \sigma\left(s ; M, M^{\prime}\right) \frac{<0|j| M^{\prime}>d M^{2}}{Q^{2}+M^{\prime 2}}
$$

In Eq. (1) $j$ denotes the current of the probe interaction. For a virtual photon, $j$ is the electro-magnetic current and Eq. (1) gives so called Gribov's formula (see Ref.[2]).

In this work we use Eq. (1) the current which is equal to the trace of QCD energymomentum tensor

$$
j=\theta_{\mu}^{\mu}=\frac{\beta(g)}{2 g} F^{\alpha \alpha \beta} F_{\alpha \beta}^{a},
$$

where the QCD Lagrangean has a form $\mathcal{L}=-\frac{q^{2}}{4} F^{\alpha \alpha \beta} F_{\alpha \beta}^{a}$. This current is a perfect probe of the gluon structure function as was pointed out in Ref.[3].

To nnake use of the formula of Eq. (1), we introduce a separation parameter $M_{0}$ in integration over masses in Eq. (1) and consider two different region in integration: "soft" and "hard". An observation which is most important for the following is that in the soft region, the matrix element $<0\left|\theta_{\mu}^{\mu}\right| M>$ can be fixed by anomaly matching. Indeed, at small invariant mass an acurate description of QCD is given by the effective chiral Lagrangean

$$
\mathcal{L}=\frac{f_{\pi}^{2}}{4} \operatorname{tr} \partial_{\mu} U \partial^{\mu} U^{\dagger}+\frac{1}{4} m_{\pi}^{2} f_{\pi}^{2} \operatorname{tr}\left(U+U^{\dagger}\right)
$$


where $U=\exp \left(2 i \pi / f_{\pi}\right), \pi \equiv \pi^{a} T^{a}$ and $T^{a}$ are the $S U(2)$ generators normalized by tr $T^{a} T^{b}=$ $\frac{1}{2} \delta^{a b}$. It is easy to see $[4,5]$ that Eq. (3) leads to a beautiful result in the chiral limit of QCD:

$$
\left\langle 0\left|\theta_{\mu}^{\mu}\right| \pi^{+} \pi^{-}\right\rangle=M^{2} \text {. }
$$

Therefore, the matrix element $\left\langle 0\left|\theta_{\mu}^{\mu}\right| M\right\rangle$ is fixed by nonperturbative QCD. Of course, the two pion approximation is rather crude at $M \sim 1 \mathrm{GeV}$, but in that region one can rely on phenomenological approach, using experimental data on $\pi-\pi$ scattering as an input (see, e.g. [5] ). For "soft" processes it is reasonable to start with the assumption that the cross section in Eq. (1) is diagonal with respect to masses, namely $\sigma\left(s, M, M^{\prime}\right)=\sigma_{0} M^{2} \delta\left(M^{2}-M^{\prime 2}\right)$. This assumption is in agreement with high energy phenomenology and/or with the experimental data in which the diffractive cross sections are smaller than elastic one. It should be stressed that this assumption leads to quite good description for DIS of virtual photon.

In the chiral limit of QCD it is natural to assume that "soft" cross section $\sigma_{0}$ is equal to

$$
\sigma_{0}=\sigma\left(\pi^{+} p ; s\right)+\sigma\left(\pi^{-} p ; s\right) \text {. }
$$

Finally, collecting both, "soft" and "hard" contributions to Eq. (1), we have

$$
\begin{aligned}
x_{B} G\left(x_{B}, Q^{2}\right) & =\frac{364 \pi^{2}}{2\left(N_{c}^{2}-1\right) b^{2} \alpha_{S}^{2}\left(Q^{2}\right)} \int^{M_{0}^{2}} \frac{d M^{2} M^{4}}{\left(M^{2}+Q^{2}\right)^{2}}\left(\sigma\left(\pi^{+} p ; s\right)+\sigma\left(\pi^{-} p ; s\right)\right) \\
& +\alpha_{S}\left(Q^{2}\right) \int_{x_{B}}^{1} \frac{d x^{\prime}}{x^{\prime}} \int^{Q^{2}} \frac{\bar{M}^{2}}{\tilde{M}^{2}} x^{\prime} G\left(x^{\prime}, \tilde{M}^{2} / 4\right) .
\end{aligned}
$$

Eq. (6) is the main result of this work. The second term in Eq. (6) is the DGLAP evolution equation [6] for the gluon structure function in the region of low $x_{B}$, while the first one is the "soft" contribution to the gluon structure functiojn. It can be easily seen that Eq. (6) has the following implications for the gluon structure function:

1. The value of the separation scale $M_{0}^{2}$ is rather large, $M_{0}^{2}=4 \div 6 \mathrm{GeV}^{2}$ (see Refs. $[5,7])$. It means that we can use the DGLAP evolution equation only for $Q^{2} \gg M_{0}^{2} \approx$ $4-6 \mathrm{GeV}^{2}$;

2. The DGLAP evolution equations can be used only for the leading twist contributions. Eq. (6) gives ua a rare possibility to extract the twist contribution from the first, "soft" term. Indeed, it can be written as

$$
x_{B} G_{\text {soft }}^{L T}\left(x_{B}, Q^{2}\right)=\frac{192 \pi^{2}}{2 b\left(N_{c}^{2}-1\right) \alpha_{S}^{2}\left(Q^{2}\right)} \times \frac{1}{Q^{4}} \times 2 \sigma(\pi p ; s) \int^{M_{0}^{2}} M^{4} d M^{2}, .
$$

Therefore, one can see that Eq. (1) and /or Eq. (6) states that the initial condition for the gluon structure function is a function which tends to zero at low $x$;

3. Eq. ( 7$)$ shows that the higher twist contribution is very essential for the gluon structure function. In spite of the fact that the contribution of Eq. $(T)$ is suppressed as $1 / Q^{1}$ in comparison with the leading twist given by the "hard" tern in Eq. (0). the simple 'stimates shows that this term is still of the order of the leading twist at $Q^{2} \sim 2$ Ge $\mathrm{F}^{2}$. For this estimates wo take $\left.\alpha_{s}=0.25 \sigma(\pi p): s\right)=25 \mathrm{mb}, b=9$ and $\mathrm{M}_{0}^{2}=4 \mathrm{Ger}^{2}$. 
To summarize, we have derived the sum rule of Eq. (6) which gives us a unique possibility to discuss the initial condition for the DGLAP evolution equation for the gluon structure function. It also fixes the higher twist contributions for this observable. It turns out that the initial gluon distribution is negligibly small at low $x$ but the higher twist contribution are quite large.

\section{References}

[1] D. Kharzeev, Yu.V. Kovchegov, and E. Levin, to appear.

[2] V.N. Gribov, Sov. Phys. JETP 30 (1970) 709.

[3] A.H. Mueller, Nucl. Phys. B335 (1990) 115.

[4] M.B. Voloshin and V.I. Zakharov, Phys.Rev.Lett. 45 (1980) 688.

[5] H. Fujii and D. Kharzeev, Phys. Rev. D60 (1999) 114039; hep-ph/9807383.

[6] V.N. Gribov and L.N. Lipatov,Sov. J. Nucl. Phys. 15 (1972) 438; L.N. Lipatov, Yad. Fiz. 20 (1974) 181; G. Altarelli and G. Parisi,Nucl. Phys. B126 (1977) 298; Yu.L. Dokshitser,Sov. Phys. JETP 46 (1977) 641.

[7] V.A. Novikov, M.A. Shifman, A.I. Vainshtein and V.I. Zakharov, Nucl. Phys. B191 (1981) 301. 


\title{
Diffractive Physics at eRHIC
}

\author{
K. Itakura ${ }^{1}$, K. Suzuki ${ }^{2}$ J. Alam ${ }^{2}$, T. Hatsuda ${ }^{2}$, and A. Hayashigaki ${ }^{2}$ \\ ${ }^{1}$ RIKEN-BNL Research Center, Brookhaven National Laboratory, Upton, \\ NY11973-5000, USA \\ ${ }^{2}$ Physics Department, University of Tokyo, Tokyo 113-0033, Japan
}

eRHIC facility opens up a variety of new regions in hadron physics. Its kinematics immediately implies that it allows us to survey the small- $x$ high parton density of nuclei by using the DIS off large nuclei, which is now being extensively discussed by many people. Here, however, we discuss other physics possibilities in eRHIC. The small- $x$ kinematics also means that eRHIC is a very useful device for the diffractive physics. In the following, we mainly consider both of the diffractive processes in the eA and polarized ep scatterings. As for physics under nuclear environment in eA collisions, there are, at least, two important directions. The first is to see purely matter effects on production/propagation of hadrons, which are never observed in fundamental processes. The second is to consider a large nucleus as an amplifier of tiny effects which could exist even in fundamental processes. Based on these, we propose the following subjects as interesting physics in eRHIC.

\section{Diffractive vector meson productions}

In the small- $x$ kinematics, amplitude of the vector meson $(V)$ production on a nucleon $(N): \gamma^{(*)}+N \rightarrow V+N$ can be given simply as $\mathcal{A} \propto \psi_{\gamma} \otimes \sigma_{q \bar{q} N} \otimes \psi_{V}$, where $\psi_{\gamma}$ and $\psi_{V}$ are $\mathrm{LC}$ wavefunctions of photon and vector mesons. The dipole cross section $\sigma_{q \bar{q} N}$ is a scattering amplitude between a $q \bar{q}$ pair and a nucleon. When one has a hard scale (such as heavy quark mass or large $Q^{2}$ ) in the process, one can evaluate $\sigma_{q \bar{q} N}$ by perturbative QCD [1]. A standard perturbative calculation is based on the small dipole approximation for a $q \bar{q}$ pair and the dipole cross section is given as $\sigma_{q \bar{q} N} \propto$ $b^{2} \alpha_{s} x G\left(x, Q^{2}\right)$ where $b$ is a transverse separation between $q$ and $\bar{q}$, and $x G\left(x, Q^{2}\right)$ is the gluon distribution in a proton. While the dipole approximation works well for $J / \psi$, it is not a good approximation for $\psi^{\prime}$ simply due to its large size $r_{\psi^{\prime}} \approx 0.8 \mathrm{fm}$. We have shown that the value of $\psi^{\prime}$ to $J / \psi$ production ratio is significantly improved by taking the multi-pole effects into account [2]. Also, the multi-pole contributions drastically affect the dipole cross section for $b>0.4 \mathrm{fm}$ [3]. For moderately large transverse size $b<1 \mathrm{fm}, \sigma_{q \bar{q} N}$ becomes much smaller than the above result and is still below the hadronic bound $\sigma \sim 20 \mathrm{mb}$ for $x=10^{-3}$. Therefore, in a sense, our calculation enlarged the region of applicability of perturbative QCD. We have also calculated the diffractive $\Upsilon(1 S, 2 S, 3 S)$ productions [2]. Since the precise data for $\Upsilon$ are not available at the moment (only 2 points at HERA), it would be better to measure it in eRHIC.

Because the deviation from the dipole approximation for $\psi^{\prime}$ case is directly related to its large size, we naively expect that this effect will be further amplified in nuclei 
(duc to multiple scattering or large ovcrlapping). However, it depends on the kinematics: whether the vector meson is formed within a nucleus or outside of it. In the diffractive process with small- $x$ kinematics, the 'formation length' of a $q \vec{q}$ pair is much larger than the nuclear radius. Hence, the vector meson is expected to be formed outside of the nucleus. If so, the matter effects should be addressed as the modification of the dipole cross section $\sigma_{q \bar{q} N}$. Usually this effect is expected to be small (color transparency), but for moderate $Q^{2}$ and if we measure the excited(2S) vector mesons in the final state, the transverse size of a $q \bar{q}$ pair is not so small and we expect some matter effects. On the other hand, if the vector meson is formed inside of a nucleus, we can further enjoy the other matter effect: the scattering of a vector meson off nucleons in a nucleus. These two different situations will be realized by changing the energy of a nucleus, which should be possible in eRHIC. Lastly, though many people already claimed, we also should emphasize that the diffractive $J / \psi$ production off nuclei is one of the best ways to extract the nuclear gluon distribution. Only eRHIC can do this experiment.

\section{2. $J / \psi$ and $\psi^{\prime}$ productions by eA collisions}

Related to, but slightly different from the above subject, let us consider the inclusive production cross section of $J / \psi$ and $\psi^{\prime}$ by eA collision, $e+A \rightarrow J / \psi\left(\psi^{\prime}\right)+X$. Formation and absorption of charmonium in hadron collisions attract considerable interests and are currently under a controversial situation whether the color octet process is really the dominant contribution. While the $J / \psi$ production in $p p$ collision allows the color octet process and is believed to be dominated by it, the main contribution in the electroproduction of charmonium $e+p \rightarrow J / \psi+X$ at low $z$ is the color singlet process. This was confirmed by the comparison of HERA experiment with theoretical calculations. Hence, use of the electron beam is advantageous to study matter effects on the propagation of charmonium in nuclei, since the propagation of the color singlet object seems to be under control. We can extract the information on the nuclear absorption of $J / \psi$ and $\psi^{\prime}$ in more reliable way. On the other hand, we do not know the absorption of the color octet objects in nuclei and have much ambiguities.

Using the standard technique [4] and the $J / \psi-N, \psi^{\prime}-N$ absorption cross section calculated by the model based on perturbative QCD [3], we find $\sigma(e+A \rightarrow J / \psi) / A \sigma(e+$ $p \rightarrow J / \psi)=0.75$ and $\sigma\left(e+A \rightarrow \psi^{\prime}\right) / A \sigma\left(e+p \rightarrow \psi^{\prime}\right)=0.54$ for Au target. Here, $J / \psi$ coming from decays of $\chi$-states are not included in this estimate, but can be incorporated.

In addition to the conventional nuclear absorption, a new mechanism of $\psi^{\prime}$ suppression is proposed due to the mass reduction of $D \bar{D}$ mesons [5]. QCD sum rule calculation tells us that the mass of $D$-meson at normal nuclear mater density is reduced by about $100 \mathrm{MeV}$. It indicates $\psi^{\prime}$ can decay into $D \bar{D}$ inside the nucleus, because $D \bar{D}$ threshold comes below the mass of $\psi^{\prime}$. A crude estimate shows that. with momentum of a produced $\psi^{\prime}$ being about $10 \mathrm{GrV}$, the suppression factor 
$\sigma\left(e+A \rightarrow \psi^{\prime}\right) / A \sigma\left(e+p \rightarrow \psi^{\prime}\right)$ decreases to $\approx 0.2$. Observation of such effects may be possible at the eRHIC facility.

\section{Spin-dependent diffractive processes}

Study of the spin-dependent diffractive process is of special interest for various hadron properties. It has been argued that the diffractive production of $J / \psi$ in the longitudinally polarized ep collision, $\vec{\gamma}+\vec{p} \rightarrow J / \psi+\vec{p}$, is not a good probe of the polarized gluon distribution $\Delta g\left(x, Q^{2}\right)$ of the proton [6]. In the forward case, $t=0$, the asymmetry $A_{L L}$ is shown to vanish in the non-relativistic limit within the collinear approximation. ${ }^{1}$ However, inclusion of explicit intrinsic momenta of quarks and gluons is expected to provide a finite asymmetry even at $t=0[7,8]$. If so, this process is one of the ideal tools for the measurement of $\Delta g\left(x, Q^{2}\right)$ free from the notorious color octet contribution.

In addition to the possibility of measuring the gluon polarization, the spin-dependent diffractive processes allow us to obtain many new quantities of hadrons. By observing the helicities of the final state, we can extract the helicity flip skewed parton distribution. This distribution is sensitive only to the gluons in the nucleon. On the other hand, if one can measure a complete set of helicity amplitudes, it may be possible to discuss the $D$-wave component of the vector meson wave function. Further experimental test of s-channel helicity conservation at high energy is also possible.

\section{Difference between gluonic Pomeron and pionic Pomeron}

Usually the Pomeron is believed to be a very complicated gluonic state. When the perturbative calculation is somewhat justified, the QCD Pomeron can be described as two (Reggeized) gluons and obeys the BFKL equation. This is the 'hard' Pomeron. On the other hand, it is sometimes argued that there is an another Pomeron (the 'soft' Pomeron) with different properties, and of non-perturbative origin [9]. At present, we have no definite idea how to describe the soft Pomeron. However, an interesting suggestion was made by Bjorken [10] that the soft Pomeron or high energy scattering could be described as scattering between pion clouds of each hadrons. ${ }^{2}$ This picture implies that the soft Pomeron is most likely a multipionic state. How can we distinguish the gluonic and pionic Pomerons? A possible way could be the diffractive scattering off nuclei, which completely belongs to the eRHIC physics. The pion cloud is a small distortion of the vacuum and thus should be intimately related to chiral condensate. Therefore if the vacuum property changes, the pion cloud will also get modified. Recently, it has been discussed that partial restoration of the chiral symmetry (reduction of the chiral condensate) is already

\footnotetext{
${ }^{1}$ At non-zero momentum transfer to the proton, $t \neq 0$, we expect finite asymmetry even in the nonrelativistic limit, which could reveal the spin-dependent structure of the Pomeron.

'See also Ref. [11] which partly incorporated pionic contributions in the laxdders between two gluous.
} 
seen in nuclear medium [12]. Therefore, if the pionic Pomeron propagates in nuclear medium, which is expected to occur in eA diffractive scattering, its properties will change. Also, if the gluonic Pomeron is related to the gluon condensate, we can again expect change due to medium modification of the gluon condensate. How-

ever, these two changes will be different and we could distinguish the gluonic and pionic Pomerons. Indeed, the gluon condensate is much less affected compared to the chiral condensate in nuclear medium [13].

\section{References}

[1] S. Brodsky, et al. Phys. Rev. D50 (1994) 3134.

[2] K. Suzuki, et al. Phys. Rev. D62 (2000) 031501(R).

[3] K. Suzuki and K. Itakura, in preparation.

[4] C. Gerschel and J. Huefner, Phys. Lett. B207 (1988) 253.

[5] A. Hayashigaki, "Mass modification of D-meson at finite density in QCD sum rule", nucl-th/0001051, Phys. Lett. B in press.

[6] M. Vanttinen and L. Mankiewicz, Phys. Lett. B434 (1998) 141.

[7] M. Vanttinen and L. Mankiewicz, Phys. Lett. B440 (1998) 157.

[8] A. Hayashigaki, et al. work in progress.

[9] A. Donnachie and P.V. Landshoff, Phys. Lett. B437 (1998) 408.

[10] J. D. Bjorken, "Multiparticle Dynamics 1997: Concluding Talk", hep-ph/9712240.

[11] D. Kharzeev and E. Levin, Nucl. Phys. B578 (2000) 351.

[12] T. Hatsuda, T. Kunihiro, and H. Shimizu, Phys. Rev. Lett. 82 (1999) 2840; D. Jido, T. Hatsuda, and T. Kunihiro, "In-medium $\pi \pi$ Correlation Induced by Partial Restoration of Chiral Symmetry", hep-ph/0008076.

[13] T. Hatsuda, Nucl. Phys. A544 (1992) 27c . 
A. Sandaoz

$B N L, 5.07 .2000$

Hard Exclusive Processes at eTHIC

Experimental Aspects

(1.) Exclusive meson production

$$
M \begin{cases}\gamma^{\prime} T \rightarrow M T & (T=p, A) \\ \text { vector meson } & J / \psi, \rho^{0}, \ldots \\ \text { psendoscalar m. } & \pi, \eta, \eta^{\prime}, \ldots\end{cases}
$$

A. on protons $\quad \Rightarrow$ OFPD's

B. on muter

B1) A dependence for VM $\Rightarrow\left\{\begin{array}{l}\text { colour tromsparang } \\ \text { colour opacity }\end{array}\right.$

Bi) $t$.dependence of VM coherent production on light nuclei $\Rightarrow$ colour trams.

(egg. $\left.\gamma^{*} d \rightarrow \rho^{0} d\right)$

(II) Deeply Virtual Compton Scattering

$$
\begin{aligned}
& \gamma^{*} p \rightarrow \gamma p \\
& \Rightarrow \text { OFF's }
\end{aligned}
$$

(111.) Inelastic exclusive production

$$
\text { egg. } i^{* t} p \rightarrow \rho^{0} M \pi^{+}
$$

$\Rightarrow$ quark correlations in nucleon

193 


\section{Requirements for detector at eRHIC}

- Tracking

$\Rightarrow$ vertex + momenta of scattered electron and decay particles

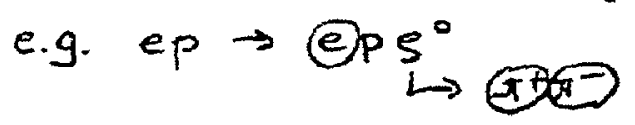

in central + mid-forward + mid-rear rregion

$$
\begin{aligned}
5<\theta<175^{\circ} \quad(|\eta|<3.1) \\
\text { with } \Delta p / p \leq 1 \%
\end{aligned}
$$

(1) accurate direct determination of Kinematics

$$
\text { (plots 182) }
$$

(2) reasonable acceptance (plot 3)

- Hermetic detector both for charged and nectral part. Small cross sections vs. large inclusive backgrand

a) topology of event

b) cut on variable $\delta=2 E_{e}-\sum_{i}\left(E-p_{z}\right)_{i}$

te detected particles

- $e / \mu / h$ separation 


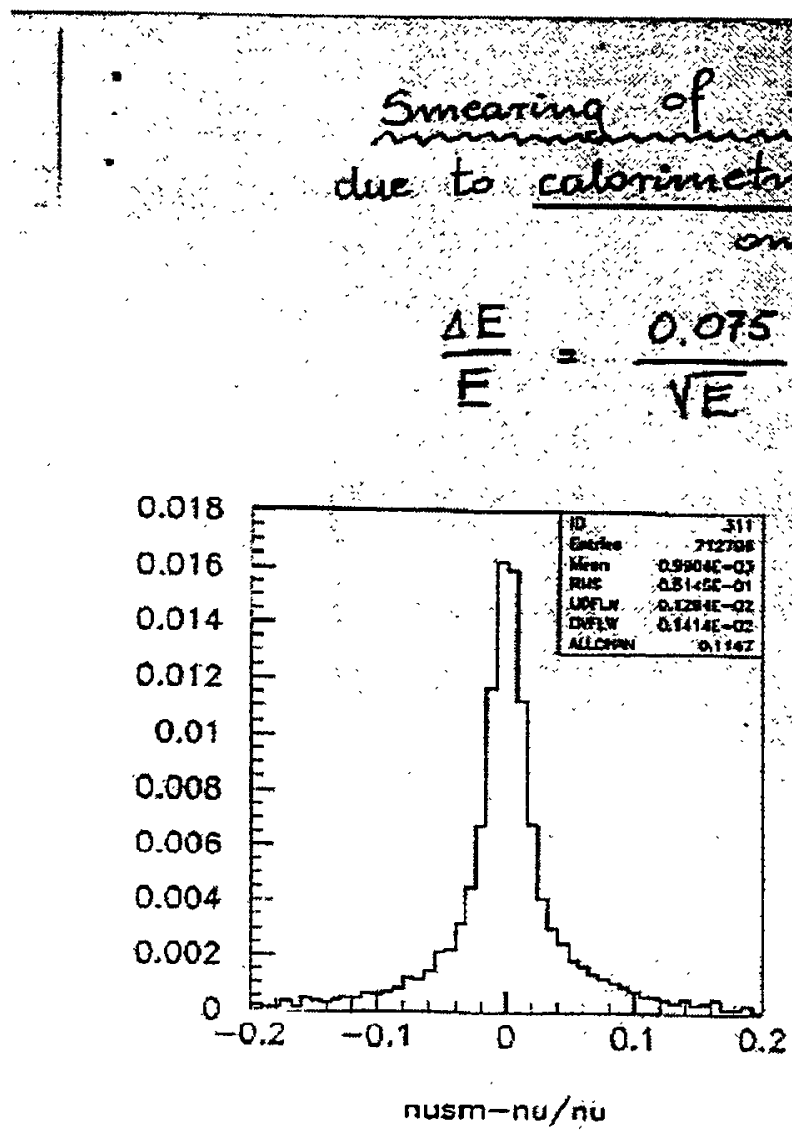

0.0 .025

(SPACAL of $H 1$ )
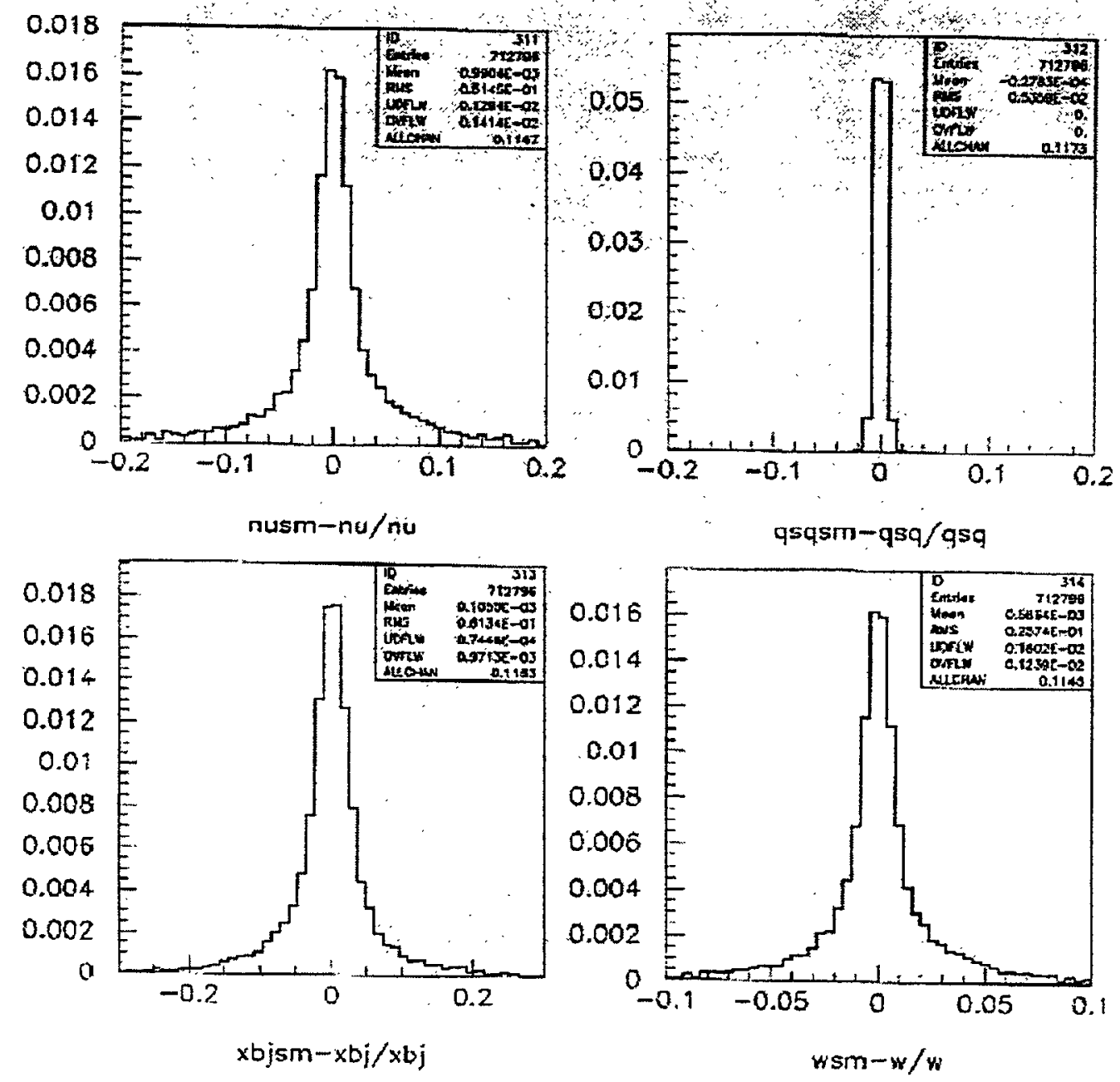


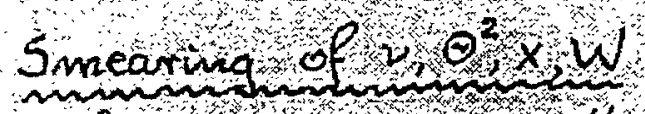

for coustrained nethod, $E_{e}=\frac{2 E_{e}-\left(E-p_{p}\right)_{M}}{1-\cos \theta_{e}}$

effects of all measured variables anduded
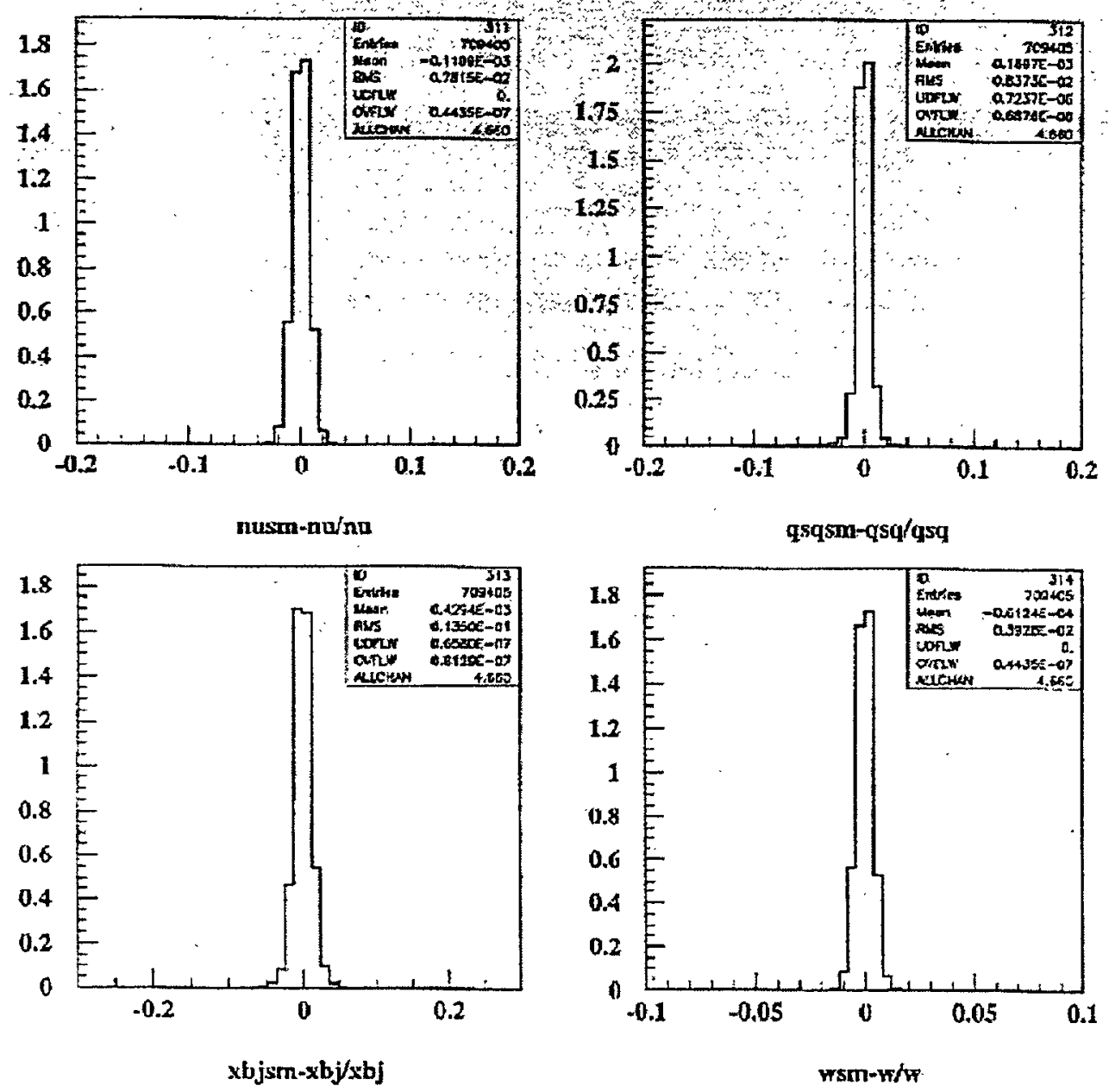


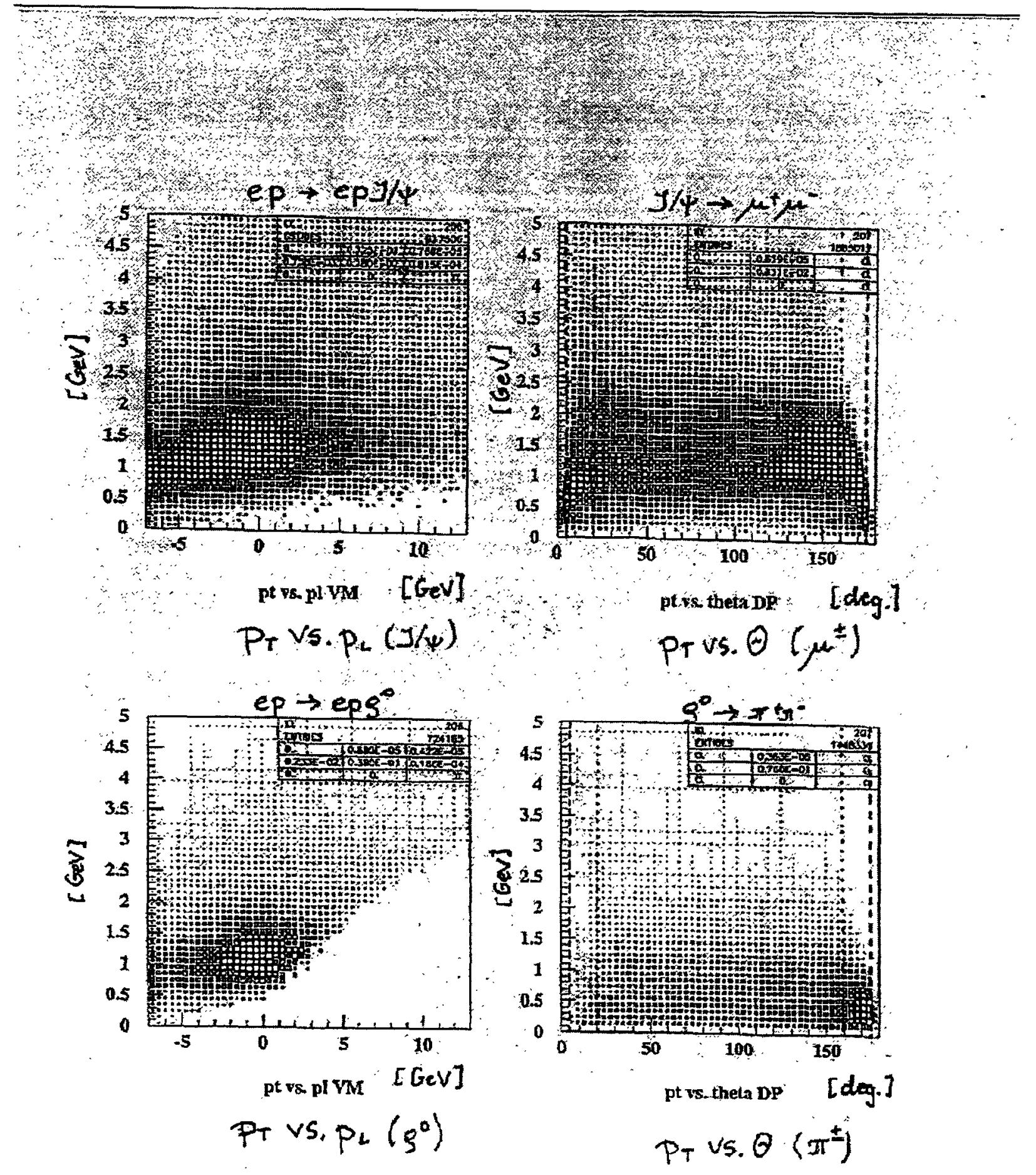




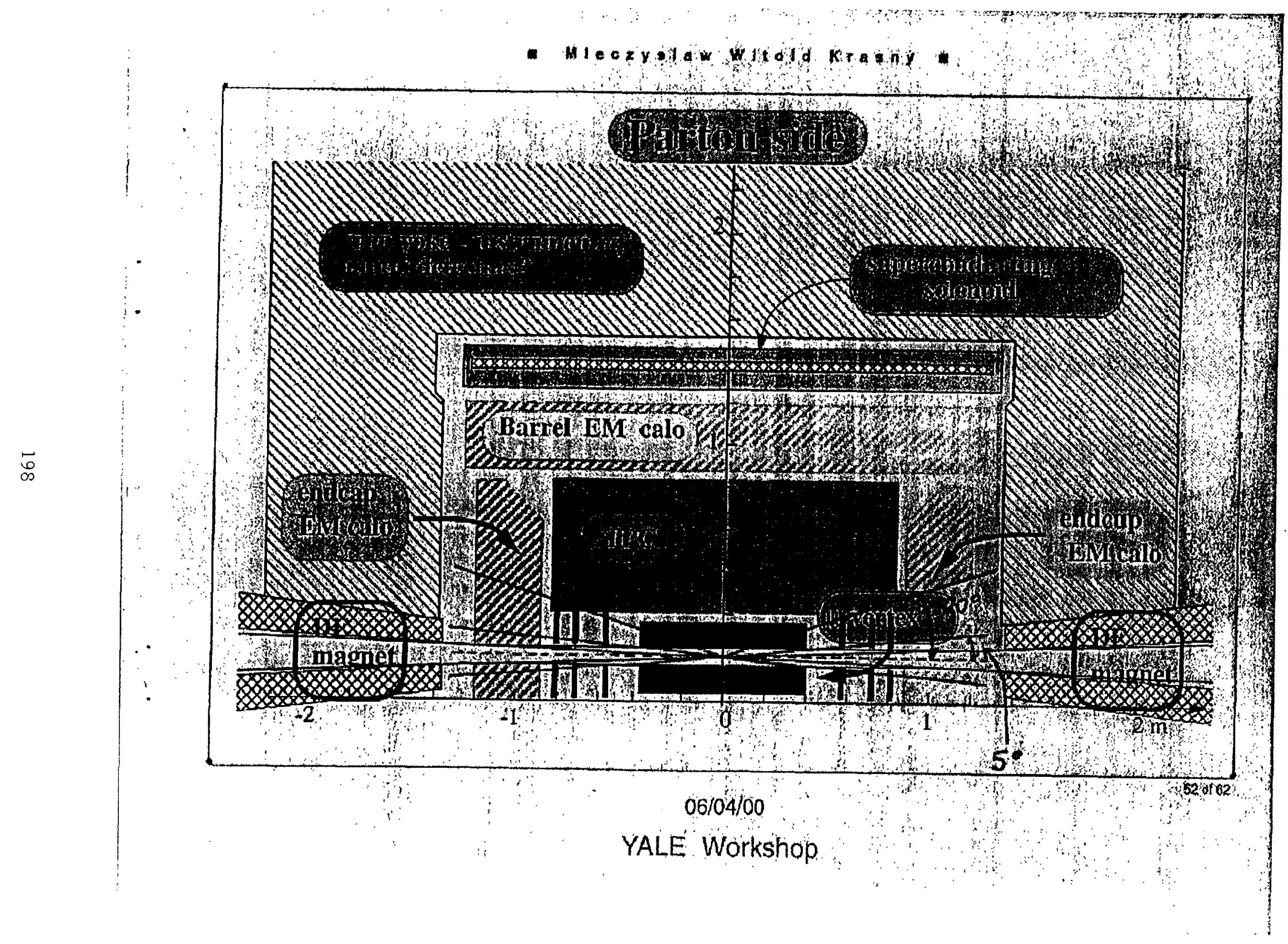


- Loading particle spectrometer (LPS)

(1) indepandent measurement of 2 hopefilly more accurafe than from central delector

(2) suppresion of nuctcon dissociation and ofther non-exdusive backgrounds

Characteristic $t$ aind $\theta_{\text {LP }}^{\mathrm{L} \text { for different processes }}$ for $10+100 \mathrm{GeN}$ collisions

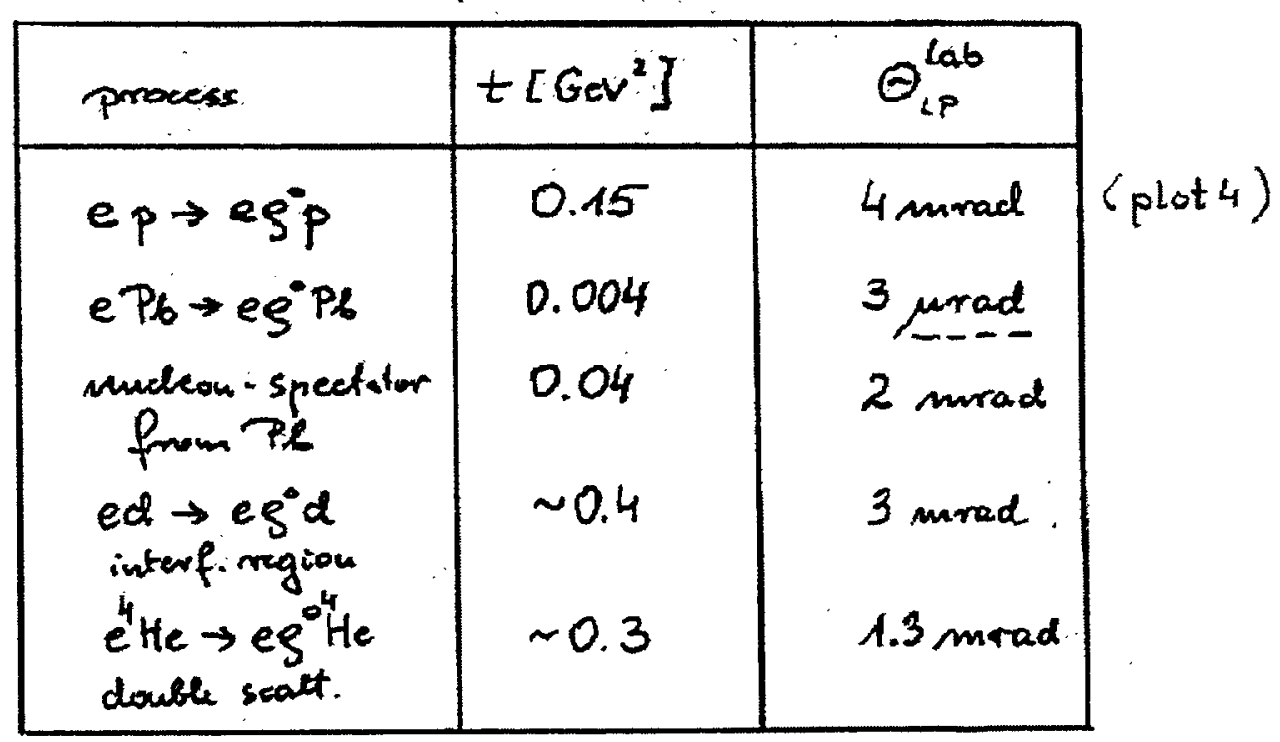

Acseptance and resolutions of LPS at eRHIC study has started recuntly

(w. Guryn, A.S.)

199 


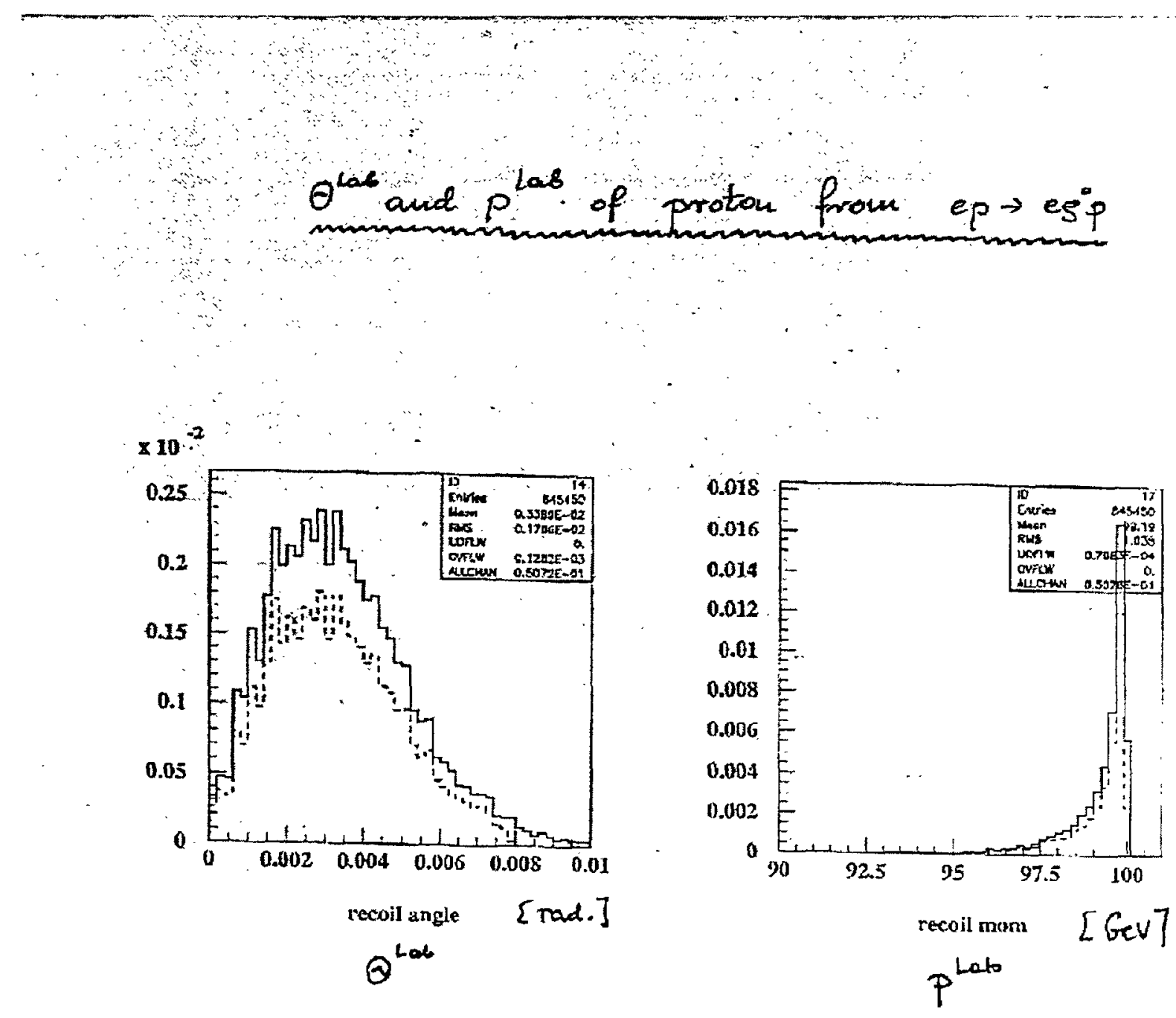

solid lines - generated events dashed lines - acceptance for central detector includea 
Reference points for LPS

ad 1.

$\langle\delta t\rangle ఐ\langle t\rangle$ as determined from central detect. for various. VM's and beams for constraimscl meth. (at $10 \times 100 \mathrm{GeV}$ )

$$
\begin{aligned}
& \text { protons lead } \\
& s^{0} \quad 0.008 \bigcirc 0.15 \quad 0.004 \bigcirc 0.05^{(*)} \\
& \begin{array}{lllllllll}
J / \psi & 0.016 & \Xi & 0.22 & 0.008 \otimes & 0.06^{(*)}
\end{array}
\end{aligned}
$$

(*) coherent +quasielastic

ad 2.

for ZEUS LPS

background from diffractive dissociation for $s$ plestopraduction

is $0.2 \%$

vs. 11\% without LPS

201 
Acceptances and Kinematic range for central + mict-forward + mid -rear detector

Previous analysis (A.S. presentation at tole matin) assuming Zeus -like defector

$$
\begin{aligned}
19.7^{\circ}< & \theta_{c}<176.5^{\circ} \\
& P_{D P}^{\top}>160.3^{\circ}
\end{aligned} \quad(|\eta|<1.75 \mathrm{GEV} \quad
$$

found possible kinematic range at $100 \mathrm{CW}$

$$
\left\{\begin{array}{l}
1<Q^{2}<25 \mathrm{Gev}^{2} \\
10<w<40 \mathrm{Gew} \\
40 \leq 2 \leq 800 \mathrm{GeN} \\
0001<x<0.1
\end{array}\right.
$$

Above range is limiting exploratory potential at small $x$

In present analysis detector with extended acceptance assumed

$$
5^{\circ}<\theta_{D P}<175^{\circ} \quad(|\eta|<3.1)
$$

With such detector possible Kinematic range is

$$
\begin{aligned}
1 & <Q^{2}<50 \mathrm{GeV}^{2} \\
10 & <W<60 \mathrm{GeV} \\
40 & \approx v<1800 \mathrm{GeV} \\
0.0003 & <x<0.16
\end{aligned}
$$

(plot 5\&6)

202 

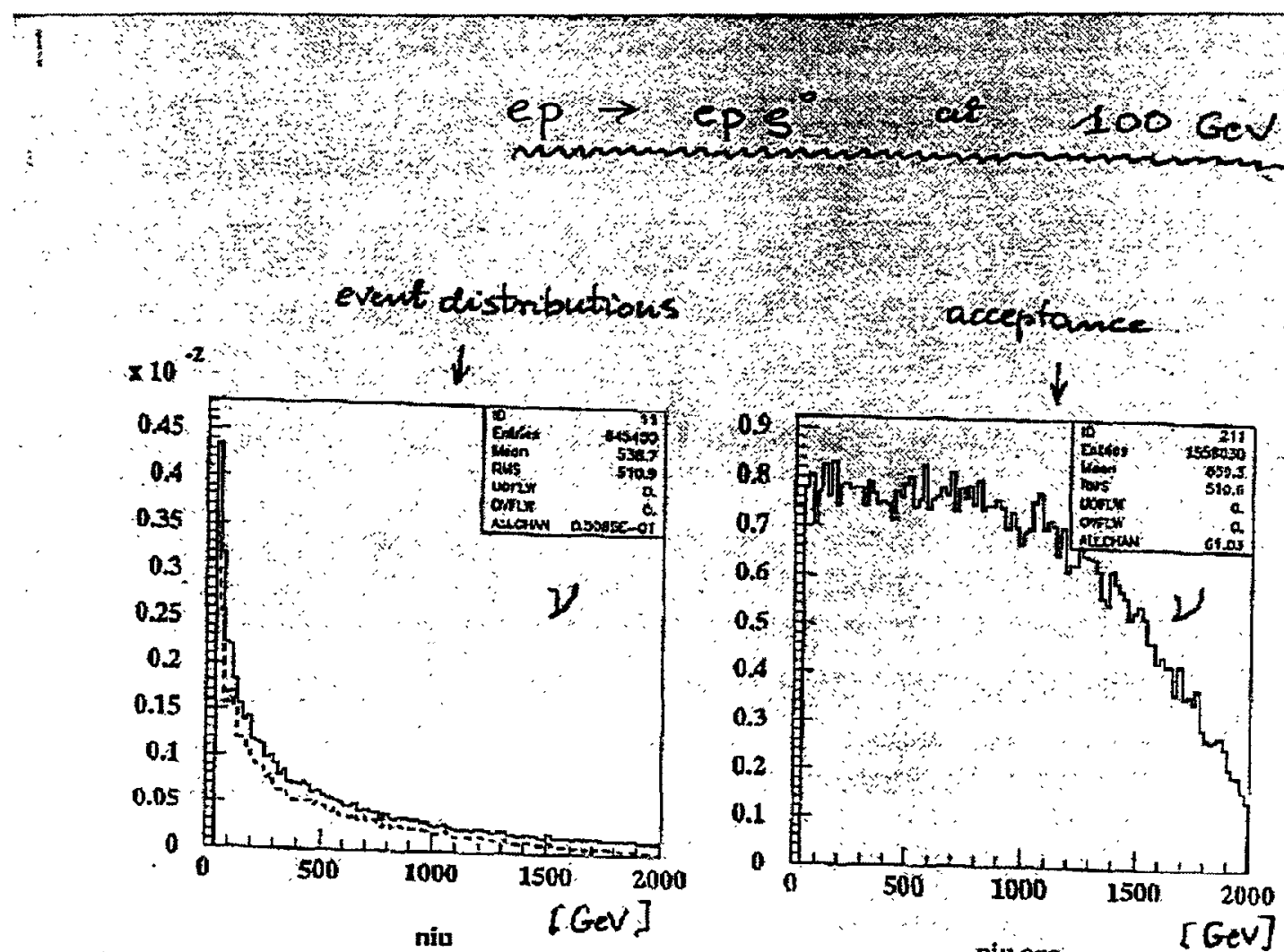

eventedistributions
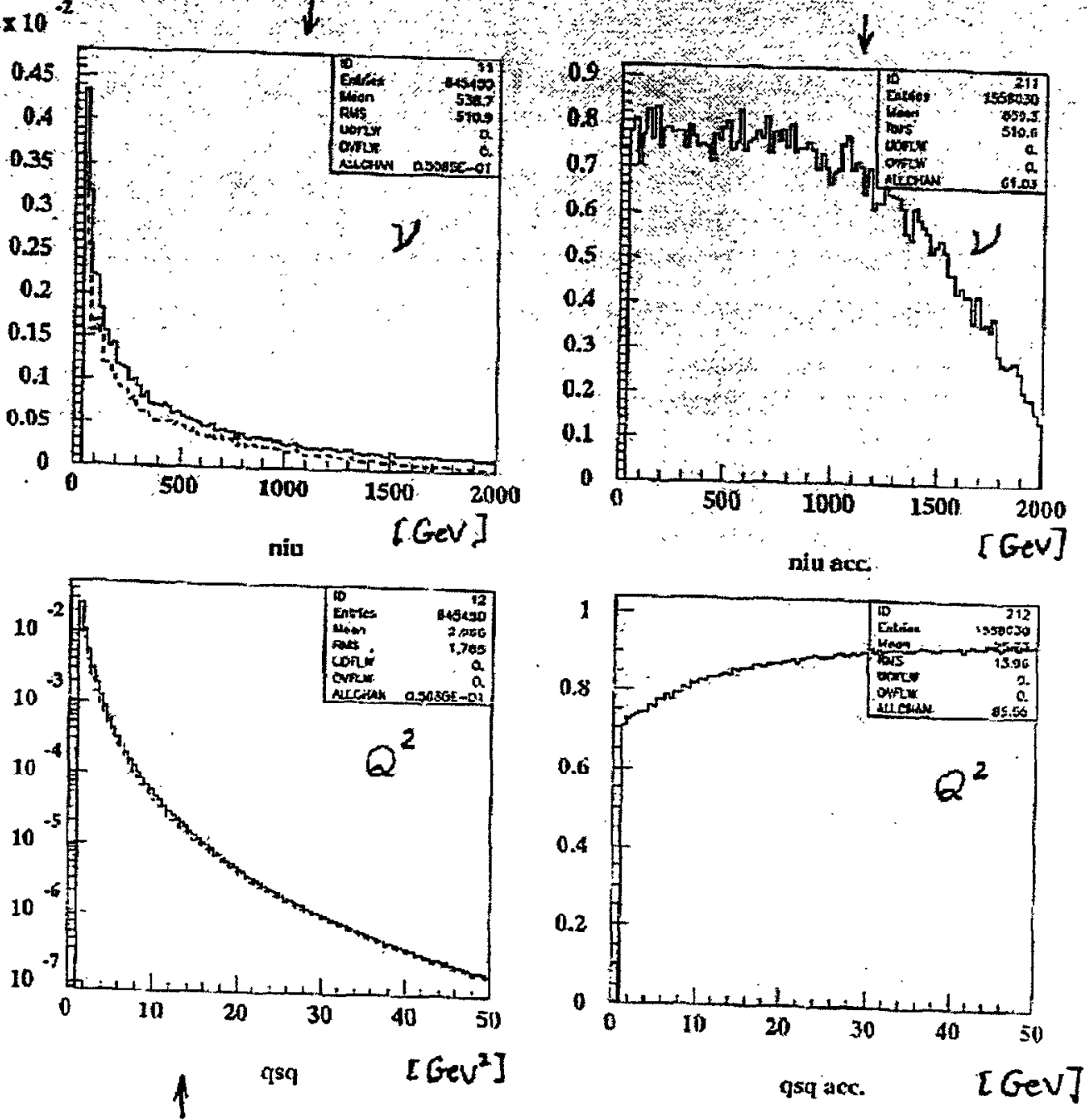

- generated

...-- curepted 

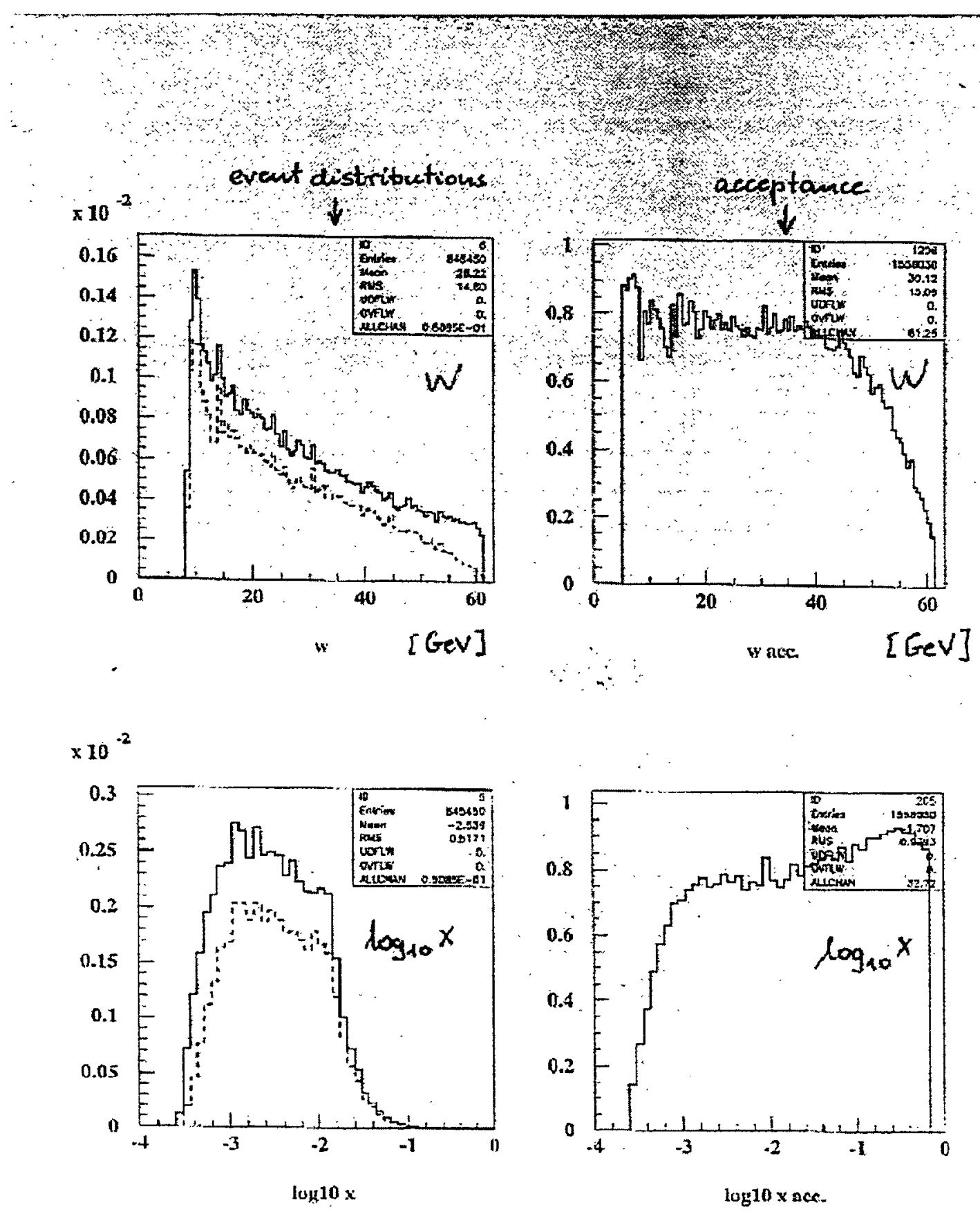
- alaczyelaw Wltold Kratiny
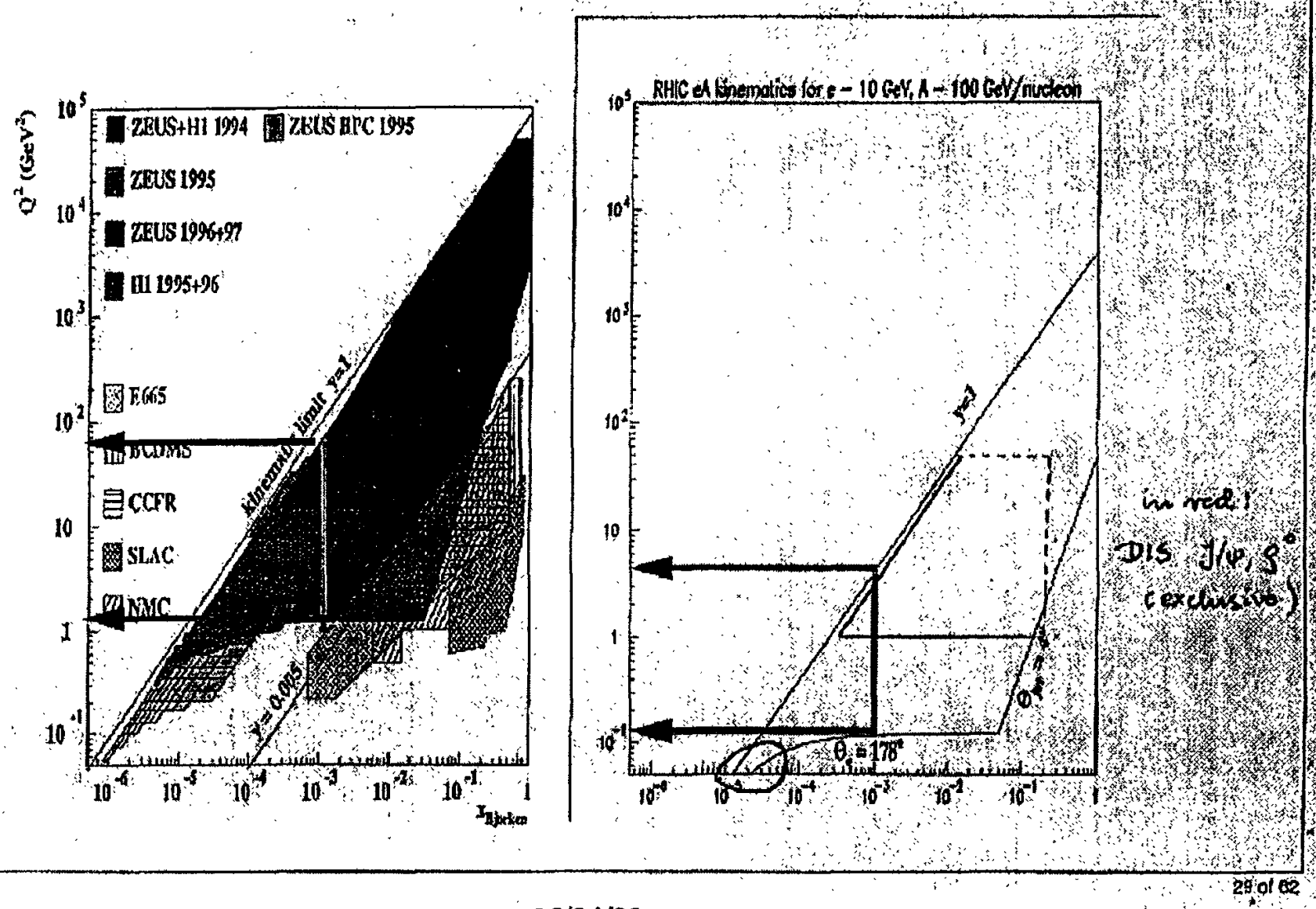

06/04/00

YALE Workshop 


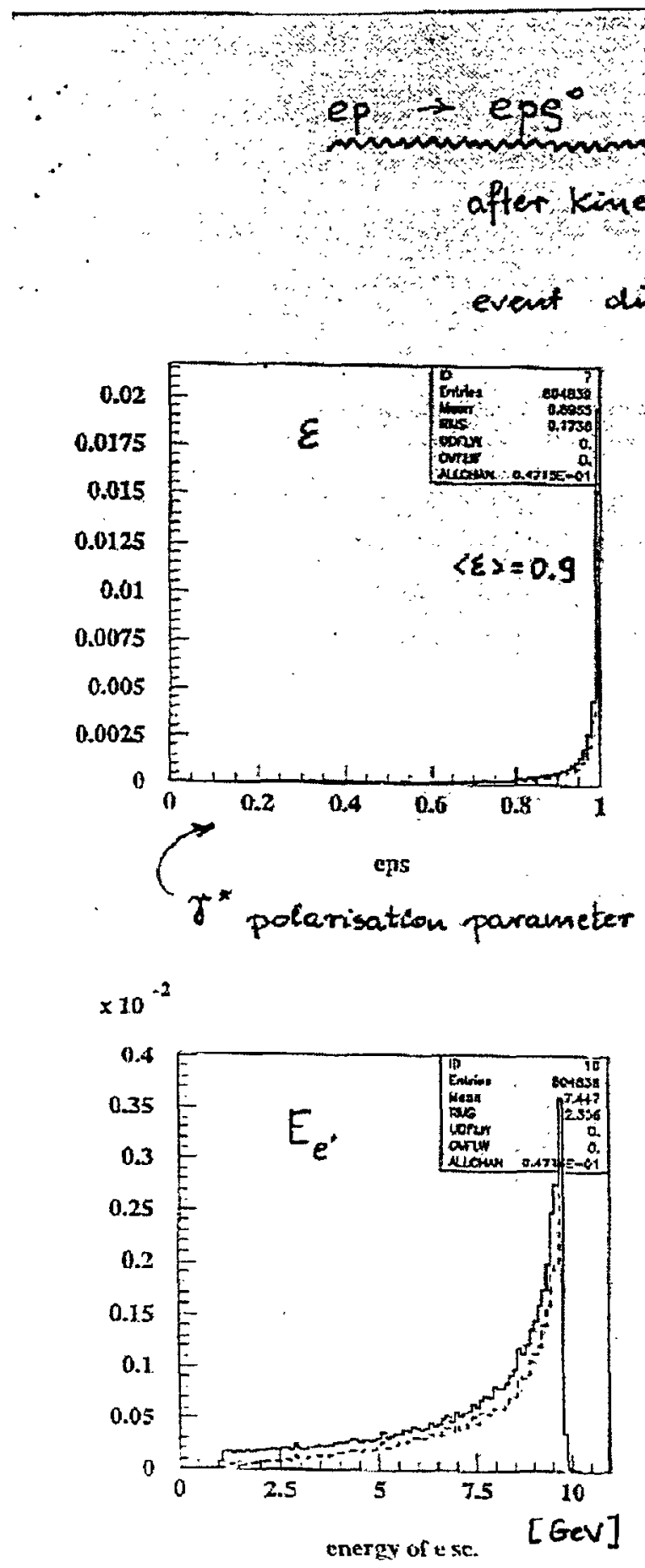

at $100 \mathrm{GeV}$

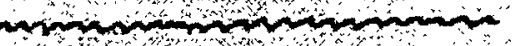

rematiose cuts 


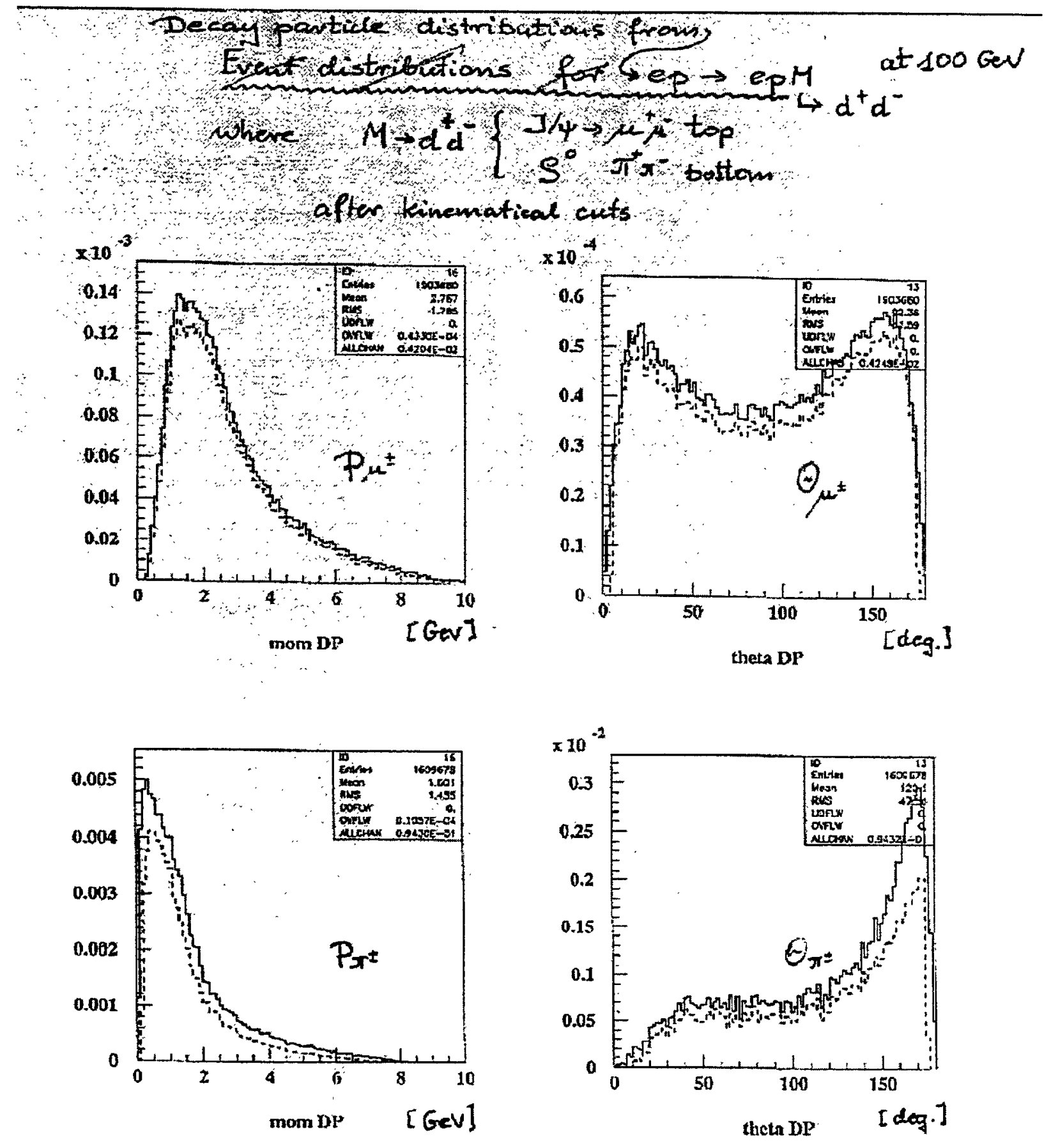


Kinematical smearing

for central and mid-forwand/rear detectors

- decay particles - resolutions fur ZEUS CTD

$$
\begin{aligned}
& \frac{\sigma_{P_{T}}}{P_{T}}=0.0058 p_{T} \oplus 0.0065 \oplus 0.0014 / P_{T} \\
& \sigma_{\eta}=0.0015 \oplus 0.0017 / P_{T} \\
& \sigma_{\varphi}=0.0006 \oplus 0.002 / P_{T}
\end{aligned}
$$

assume valid also for $5<\theta<20^{\circ} \& 160<\theta<175^{\circ}$

- scattered electrons

$$
\begin{aligned}
& \sigma_{\theta}=\sigma_{\varphi}=0.0003 \\
& E_{e^{\prime}}-\text { from constrained method } \\
& \qquad\left(E_{e^{\prime}}=\frac{2 E-\left(E-p_{2}\right)_{M}}{1-\cos \theta_{c}}\right)
\end{aligned}
$$

208 


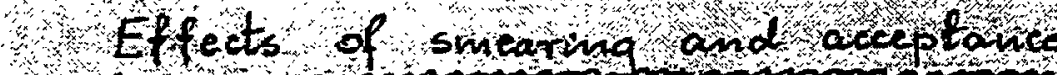

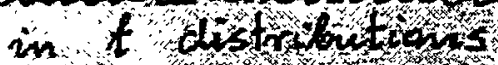

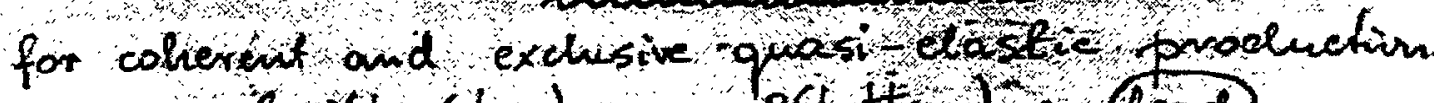
of $9 / \psi$ (top) ar $g$ (bottonn) ondead)

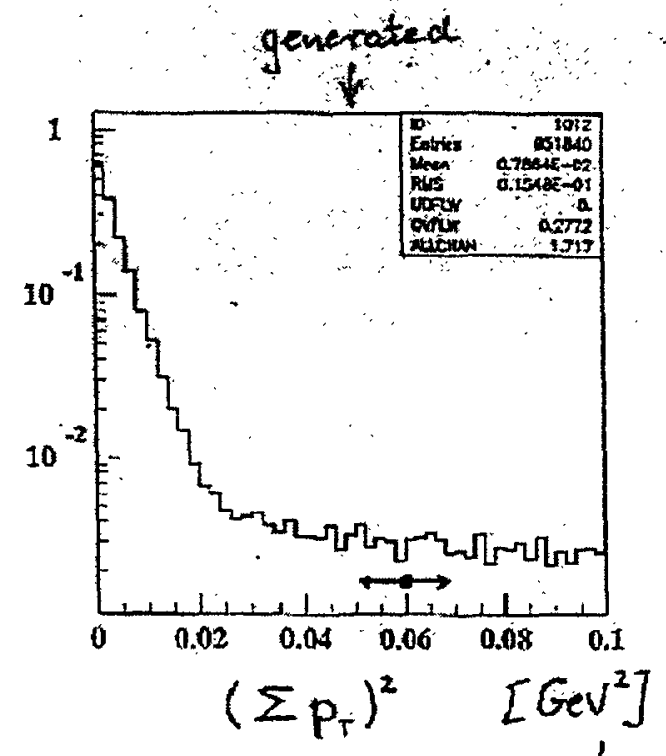

accept 1 sinearcel geiverated

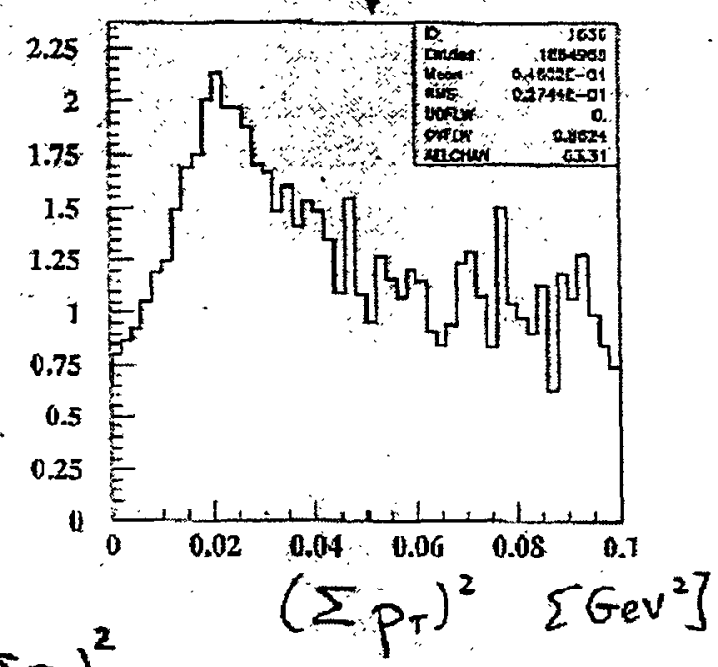
$t=\left(\Sigma p_{T}\right)^{2}$
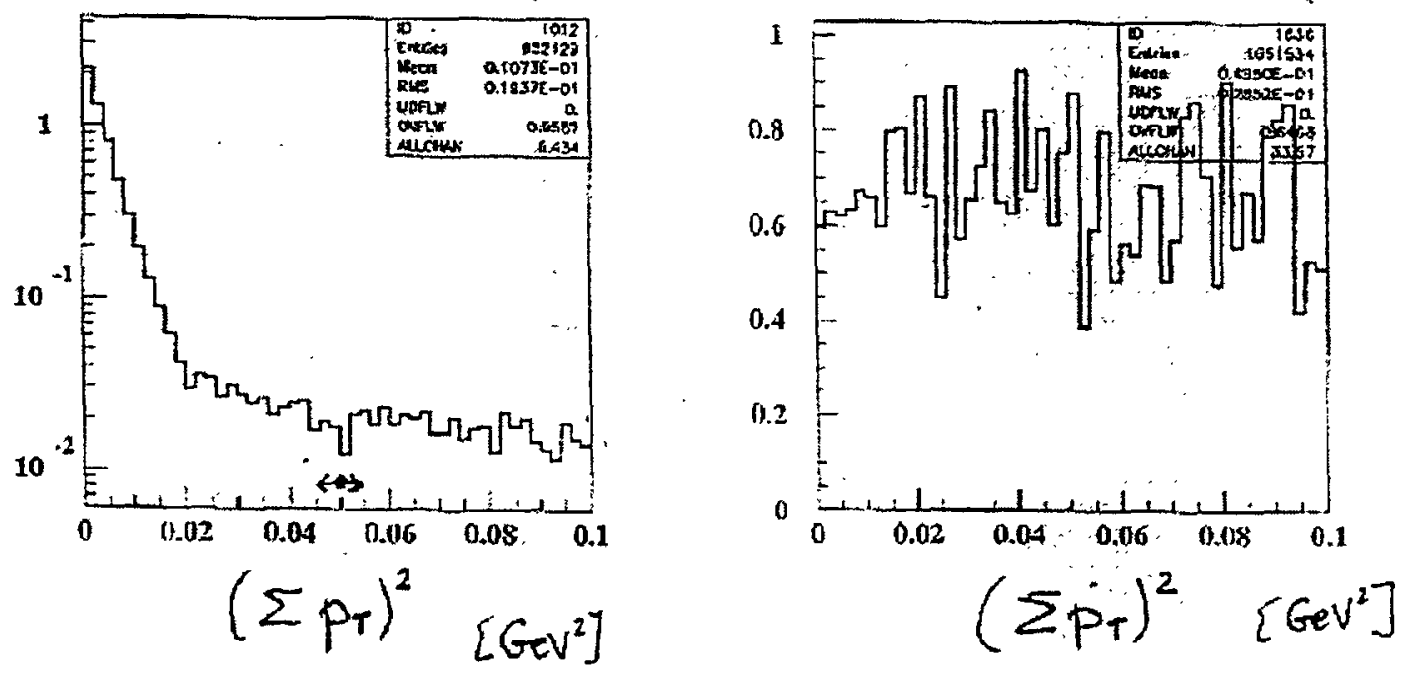

in red ruaguitude of sucearing 
lead, $100 \mathrm{God}$

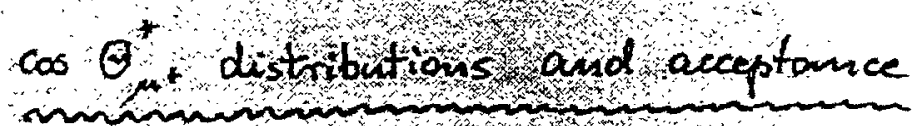

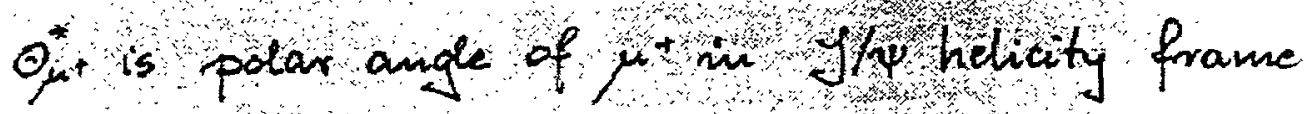
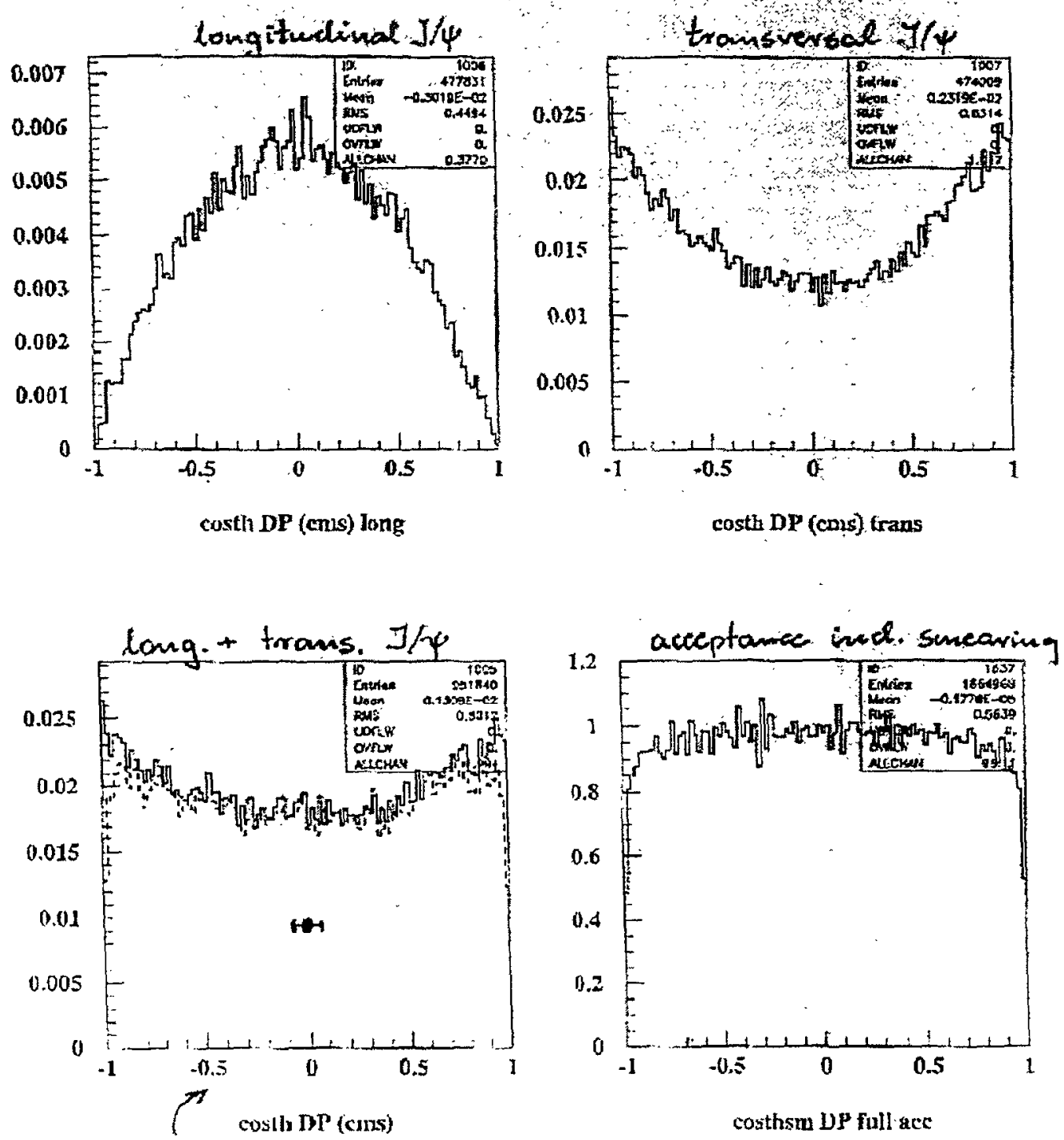

- genserated, -.--accepted tsmear. in red magnitude of smeaning in $\theta_{\mu \mathrm{f}}$ 

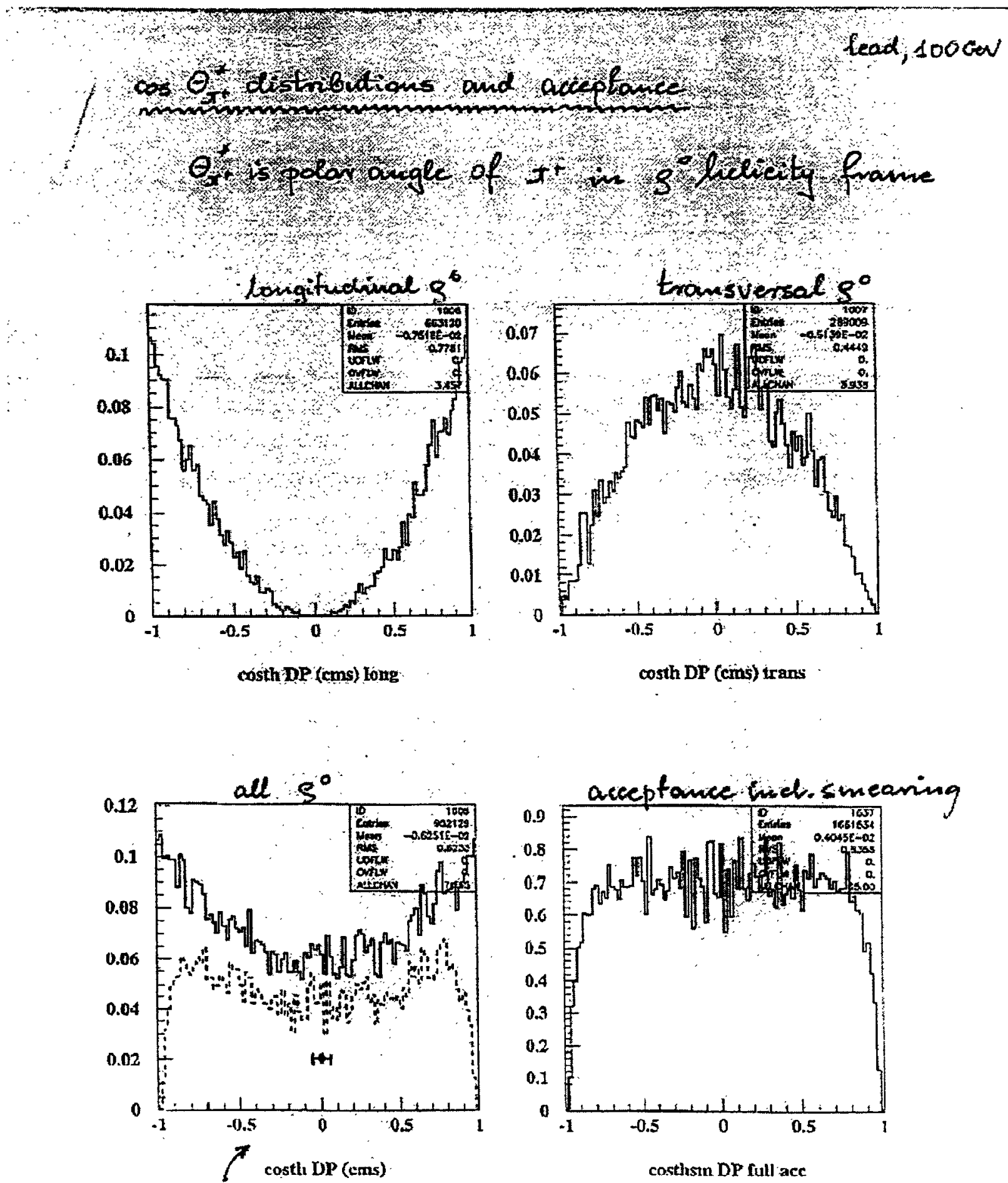

- generated, -.-- smeared + accepts in red magnitude of smeraning in $\partial_{\pi}$. 


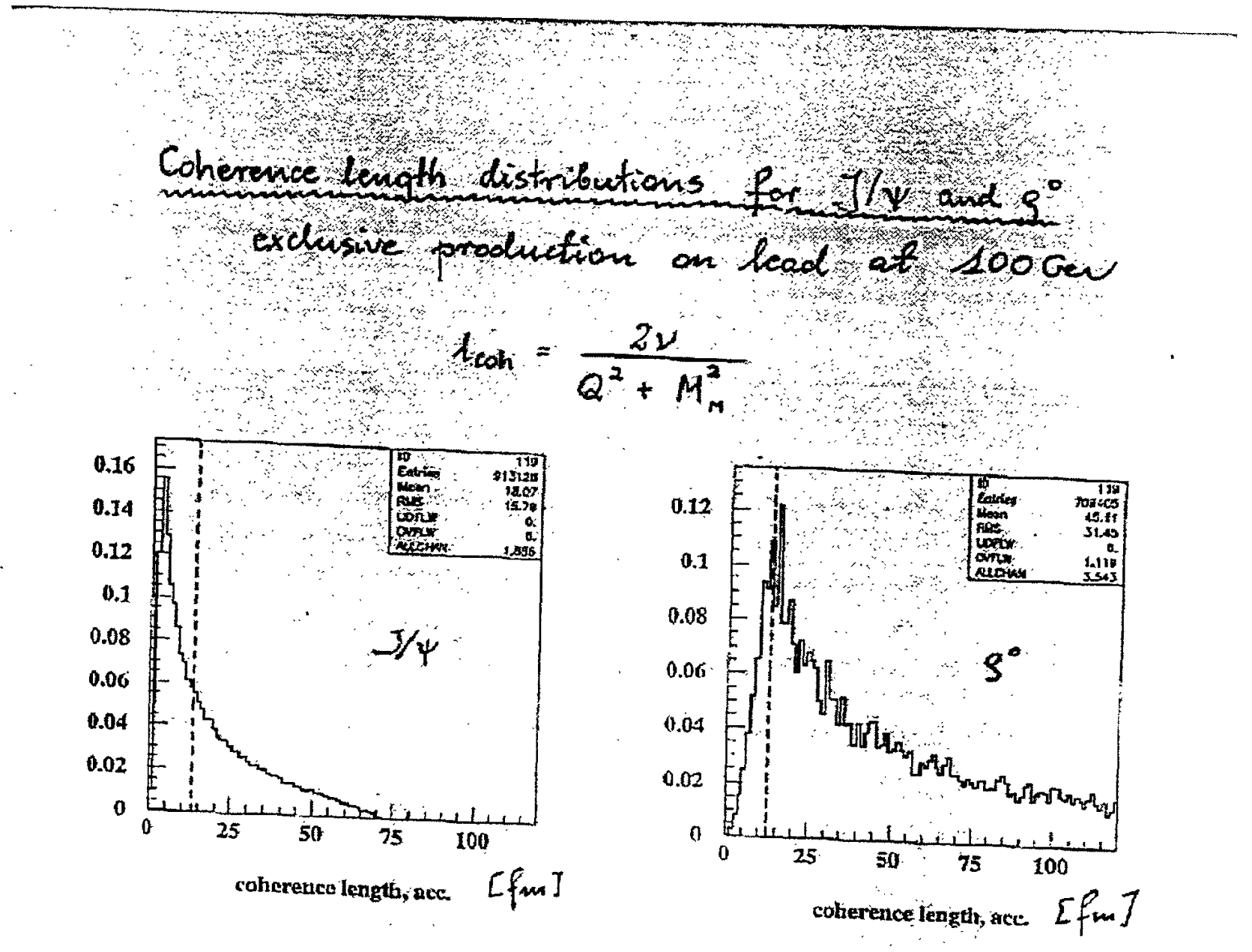


Expected rates

for $J / 4$ and 9 production on protons and lead/gold in $10 \mathrm{GeV}+100 \mathrm{GeV}$ collisions

Assumed integrated luminosities per year

for $\frac{e p}{e A w} \quad \int \mathcal{L}=\frac{330 p^{-1}}{10 p b^{-1}}$

In kinematic range $\quad \begin{aligned} & 1 \\ & 10<W<50 \mathrm{~W} \\ & 10\end{aligned}$

$$
\begin{aligned}
& \sigma_{e p \rightarrow \text { e gp }}=5.2 \mathrm{mb} \\
& \sigma_{\text {ep } \rightarrow \text { eystp }}=0.2 \mathrm{mb}
\end{aligned}
$$

for cross sections on heavy manche assumed

$$
A_{\text {eff }}^{\text {edh }}=A_{\text {eff }}^{\text {inc }}=A^{\alpha}
$$

where $\alpha=1$. for $J / 4$

$\alpha=0.75$ for $\rho^{\circ}$

Nimbler of accepted events

\begin{tabular}{|c|c|c|}
\hline & P & An $/$ Pb \\
\hline$J / 4$ & 60,000 & $1,800,000$ \\
$S^{\circ}$ & $1,070,000$ & $4,140,000$ \\
\hline
\end{tabular}

213 
Statistical precision in $x$ and $Q^{2}$ bins

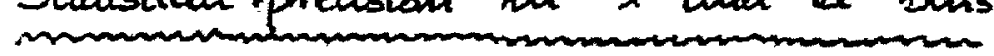
for $9 / 4$ production

\begin{tabular}{|c|c|c|c|c|}
\hline$x$ bins & $\langle x\rangle$ & $\begin{array}{c}\left\langle Q^{2}\right\} \\
{\left[G v^{2}\right]}\end{array}$ & $\begin{array}{c}\frac{\Delta \sigma}{\sigma}(e p) \\
{[\%]}\end{array}$ & $\begin{array}{c}\frac{\Delta \sigma}{\sigma}\left(e^{T B} / A \mu\right) \\
{[\%]}\end{array}$ \\
\hline $0.0003-0.001$ & 0.0007 & 1.6 & 1.1 & 0.2 \\
$0.001-0.0025$ & 0.0017 & 2.5 & 0.9 & 0.2 \\
$0.0025-0.005$ & 0.0036 & 3.7 & 0.9 & 0.2 \\
$0.005-0.01$ & 0.007 & 4.9 & 1.0 & 0.2 \\
$0.01-0.025$ & 0.016 & 7.5 & 1.0 & 0.2 \\
$0.025-0.16$ & 0.05 & 13.7 & 1.4 & 0.3 \\
\hline
\end{tabular}

\begin{tabular}{|c|c|c|c|c|c|}
\hline$Q^{2}$ & bins & $\begin{array}{l}\left\langle Q^{2}\right\rangle \\
{\left[G \in V^{2}\right]}\end{array}$ & $\langle x\rangle$ & $\frac{\Delta \sigma}{\sigma}(e p)$ & $\begin{array}{c}\frac{\Delta \sigma}{\sigma}\left(e P \& / A_{m}\right) \\
{[\%]}\end{array}$ \\
\hline & -2 & 1.4 & 0.003 & 0.7 & 0.1 \\
\hline & -4 & 2.8 & 0.006 & 0.8 & 0.1 \\
\hline & -8 & 5.6 & 0.011 & 0.9 & $=0.2$ \\
\hline 8 & -15 & 10.7 & 0.02 & 1.3 & 0.2 \\
\hline 15 & -25 & 19.0 & 0,035 & 2.1 & 0.4 \\
\hline & -50 & 33.7 & 0.06 & 2.8 & 0.5 \\
\hline
\end{tabular}


Statistical precision in $x$ and $Q^{2}$ bins

for 3 production

\begin{tabular}{|c|c|c|c|c|}
\hline$x$ bins & $\langle x\rangle$ & $\begin{array}{l}\left\langle Q^{2}\right\rangle \\
{\left[\operatorname{Gev}^{2}\right]}\end{array}$ & $\begin{array}{c}\frac{\Delta \sigma}{\sigma}\left(e_{p}\right) \\
{[\%]}\end{array}$ & $\begin{array}{c}\frac{1 \sigma}{\sigma}(\mathrm{eP} b / \mathrm{Am}) \\
{[\%]}\end{array}$ \\
\hline $0.0003-0.001$ & 0.0007 & 1.4 & 0.2 & 0.1 \\
\hline $0.001-0,0015$ & 0.0016 & 1.7 & 0.2 & 0.1 \\
\hline $0.0025-0.005$ & 0.0036 & 2.0 & 0.2 & 0.1 \\
\hline $0.005-0.01$ & 0.007 & 2.1 & 0.2 & 0.1 \\
\hline $0.01-0.025$ & 0.014 & 2.9 & 0.3 & 0.1 \\
\hline $0.025-0.16$ & 0.4 & 7.3 & 0.7 & 0.4 \\
\hline
\end{tabular}

\begin{tabular}{|c|c|c|c|c|}
\hline$Q^{2}$ bins & $\begin{array}{c}\left.Q^{2}\right\rangle \\
{\left[G_{Q} v^{2}\right]}\end{array}$ & $\langle x\rangle$ & $\begin{array}{c}\frac{\Delta \sigma}{\sigma}(e p) \\
{[\%]}\end{array}$ & $\begin{array}{c}\frac{\Delta \sigma}{\sigma}\left(e P_{k} / A_{w}\right) \\
{[\%]}\end{array}$ \\
\hline $1-2$ & 1.4 & 0.0036 & 0.1 & 0.1 \\
\hline $2-4$ & 2.7 & 0.007 & 0.2 & 0.1 \\
\hline $4-8$ & 5.3 & 0.01 & 0.4 & 0.2 \\
\hline $8-15$ & 10.3 & 0.02 & 0.9 & 0.5 \\
\hline $15-25$ & 18.5 & 0.036 & 1.9 & 1.0 \\
\hline $25-50$ & 32.5 & 0.06 & 3.5 & 1.9 \\
\hline
\end{tabular}


Conclusions

- High-accurary fast tracking detectors (egg. driftchamterstsilion) covering the range $5<\theta<175^{\circ} \quad(|\eta|<3.1)$ essential for $g / \psi$ and $\rho^{\circ}$ DIS exclusive production ranges $5<\theta<20^{\circ}$ \& $160<\theta<175^{\circ}$ correlate with the lowest $x$ range

- High statistical accuracy $(\sim 0.1 \div 2 \%)$ for $\sim 2.5$ week with minimal proposed instantenous luminosities

- Good resolutions in $Q^{2}, x, t, \cos \Theta_{D p}^{*}$ are feasible

Next om the list

- Feasibility studies for LPC

- Defector requirements for DVCS

- Defector requirements far $J / 4$ exclusive photoproduction (and low $Q^{2}$ production)

216 


\title{
Hard Exclusive Processes at eRHIC - Experimental Aspects -
}

\author{
A. Sandacz \\ Sołtan Institute for Nuclear Studies, Warsaw, Poland
}

\section{Introduction}

In recent years studies of exclusive processes in high energy scattering of leptons on nucleons and nuclei has become a topic of increasing interest. The motivations to study these processes were given by various speakers during the present and two previous eRHIC workshops. Here we just mention various processes which have been proposed.

(I) Exclusive meson production (EMP) $\gamma^{*} T \rightarrow M T$.

Here $\mathrm{M}$ denotes a produced particle, either vector or pseudoscalar meson, and $T$ could be either a nucleon (free or bound in the nucleus) or a whole nucleus.

From the studies of EMP on nucleon targets, both unpolarised and polarised, one expects to determine the Off Forward Parton Distributions (OFPD) in the nucleon. Studies of the $A$ dependence of exclusive vector meson (VM) production are a promissing tool to search for PQCD phenomena of color transparency and color opacity. Also studies of $t$-dependence of VM coherent production on light nuclei are motivated by color transparency.

(II) Deeply virtual Compton scattering (DVCS) $\gamma^{*} p \rightarrow \gamma p$.

For studies of OFPD's the DVCS is complementary with respect to EMP processes. Also from the theoretical point of view it is the most clean process to study OFPD's.

(III) Inelastic exclusive processes (for instance $\gamma^{*} p \rightarrow \rho^{0} n \pi^{+}$).

In the latter case the motivation is to study quark correlations in the nucleon.

In the following we concentrate on the experimental aspects for studies of exclusive processes at eRHIC. This memo complements the presentation on the same subject given during the eRHIC Summer Workshop. The copies of transparencies for that presentation are available at http://cern.ch/sandacz/eRHIC_w3/transp.hitml. 
We start by specifying detector requirements for studies of deep inelastic exclusive processes at eRHIC.

\section{Detector requirements}

\section{- Tracking}

A tracking detector to measure charged particles momenta with precision $\Delta p / p \leq 1 \%$ in the central, mid-forward and mid-rear regions, covering the range $5<\theta<175^{\circ}(|\eta|<3.1)$, will be an essential element of the experimental setup. Such a detector will allow to determine the position of the interaction vertex and of the momenta of scattered electron and produced particles.

We propose to measure electron momentum in the tracking detector rather than to determine it from calorimetric measurements because in the latter case the resolution of kinematic variables is poorer. This may be seen by comparing Fig. ${ }^{1} 3$ and Fig. 4. In Fig. 3 only smearing of the calorimetric mesurement of the electron energy was taken into account, whereas for Fig. 4 the electron energy was reconstructed using the so called "constrained method", for which energy of scattered electron could be expressed by measured momenta of produced paricles provided the events were truly exclusive. If the momentum of the scattered electron were measured directly in the tracking detector, the resolutions would be even better than these shown in Fig.4.

The angular coverage of the proposed tracking detector is wider than that of the present central tracking detectors in both HERA experiments, $20<\theta<160^{\circ}$. The gain in acceptance for exchusive $J / \psi$ and $\rho^{0}$ production due to the wider angular range is illustrated in Fig. 5; dashed green lines of different types in the plots at the right indicatc angular acceptances for the decay particles for the two mentioned coverages. By increasing the angular coverage one expends the acceptance towards the low $\mathrm{x}$ region (as will be discussed later).

In Fig. 6 both angular ranges are overlayed on the schematical drawing of a detector proposed for eRHIC. From the experimental point of view the measurements in the range $5<\theta<20^{\circ}$ and in the analogous range in the rear direction are challanging. Following ideas for the LHC detectors and also for upgrades of HERA detectors, these measurements could be done with silicon microstrip or silicon pixel detectors. These detectors are indicated in the area between vertex detector and EM calorimeter by thick lines perpendicular to the beam direction.

- Hermetic detector

We need an almost $4 \pi$ acceptance both for charged and neutral particles. This is important for studies of exclusive processes which have significantly smaller

\footnotetext{
${ }^{1}$ Figure numbers referred throughout this note correspond to the page numbers of the copies of transparencies in http://cern.ch/sandacz/eRHIC_w3/transp.html
} 
cross sections than the inclusive cross section. Therefore one may expect a significant background for exclusive events, unless one is be able to observe full topology of such events.

- Separation of electron, muons and hadrons

The minimal requirement would be to have the separation of electrons, muons and hadrons realised by EM calorimeters and muon detectors. Full particle identification in the momentum range up to about $10 \mathrm{GeV}$ would be necessary for studies of paricular channels, like exclusive production of charged pions or charged kaons.

- Leading particle spectrometer (LPS)

Detection and momentum measurement of a leading particle, proton or nucleus, which usually escapes into the accelerator beam pipe may be possible with the technique of the Roman pot spectrometer. Such measurements will provide independent determination of $t$, which is expected to be more acurate than that from the central detector. In addition the LPS will allow to suppress events from nucleon dissociation and other non-exclusive events.

The characteristic $t$ values for different processes as well as the corresponding polar angles of the leading particles in the laboratory system are given in Fig. 7 (cf. also Fig. 8). For exclusive production on protons or light nuclei the leading particle is scattered at small angles of few miliradians, which could be accesed with the Roman pot technique. For the coherent exclusive production on heavy nuclei the leading nucleus is emitted at much smaller angles of few $\mu \mathrm{rad}$ and therefore can not be detected by the LPS.

\section{Acceptance and kinematic range}

In the following we discuss the acceptance and kinematic range for deep inelastic exclusive production of $J / \psi$ or $\rho^{0}$ for $10 \mathrm{GeV}$ electron beam and hadron beam with energy $100 \mathrm{GeV}$ per nucleon. We consider only the acceptance of the extended central detector consisting of central, mid-forward and mid-rear detectors mentioned earlier. The analysis of the effects due to adding the LPS is in progress.

The results of simulations of exclusive $\rho^{0}$ production with the ZEUS-like detector were presented both for $250 \mathrm{GeV}$ proton beam and $100 \mathrm{GeV} /$ nucleon ion beam during the eRHIC workshop at Yale University. In paricular, it was realised that the kinematic range accessed with such detector may be limiting the exploratory potential at small $x$, in paricular studies of colour opacity. The present analysis suggests that with the extended central detector the good acceptance may be achieved in the kinematical range $0.0003<x<0.16$ and $Q^{2}>1 \mathrm{GeV}^{2}$, both for $\rho^{0}$ and $J / \psi$ production. Thus also the studies of color opacity will be possible.

More details on the kinematic ranges both for the extended central detector and for the ZEUS-like detector are given in Fig. 10. The generated and accepted event 
distributions as well as the acceptance as a function of $\nu, Q^{2}, W$ and $\log _{10}(x)$ are shown in Figs 11 and 12, whereas the distributions of kinematical variables for scattered electron and resonance decay muons (for $J / \psi$ ) and pions (for $\rho^{0}$ ) are presented in Figs. 14 and 15.

It is worth mentioning that measurements of $J / \psi$ (quasi) photoproduction at eRHIC (not covered in this presentation) will give access to even smaller $x$ values than for deep inelastic exclusive processes (cf. Fig.13).

\section{Kinematical smearing}

For the simulation of kinematical smearing we used the parametrisations of the resolutions of various kinematic variables from the ZEUS experiment (see Fig. 16). We assumed that the resolutions in the mid-forward and mid-rear parts of the extended central detector will match the extrapolations of the ZEUS parametrisations.

The effects of smearing and acceptance for coherent and exclusive quasi-elastic production on lead nucleus is show in Fig. 17 for $J / \psi$ (top) and $\rho^{0}$ (bottom). For an estimate of $t$ we use quite accurate approximation $t \simeq\left(\sum p_{T}\right)^{2}$, where the sum is over transverse momenta of all particles measured in the extended central detector, i.e. scattered electron and decay muons or pions. Red arrows indicate magnitude of smearing at average value of $t$. The smearing increases with $t$. The smearing in $t$ is acceptable, although in the part of the spectrum where coherent production dominates the smearing corrections will be large, especially for $J / \psi$. The resolution could be probably better, if $t$ is measured by the LPS.

Similarly we present the effect of smearing and acceptance for the angle $\theta^{*}$, which is the polar angle of positive decay particle in the parent vector meson helicity frame. In the lower part of the Figs. 18 and 19 the generated and accepted events distributions and the acceptance are shown as a function of $\cos \theta^{*}$.

The accurate determination of $\cos \theta^{*}$ is important to select samples enriched either in longitudinally or transversely polarised vector mesons, which will allow to study different cross section dependences on kinemetical variables and different $A$ dependences for mesons with different polarisations. The selections will be done by applying cuts in $\cos \theta^{*}$ as might be inferred from the upper part of the figures 18 and 19 , where $\cos \theta^{*}$ distributions are shown separately for vector mesons with longitudinal and transverse polarisation. The resolutions in $\cos \theta^{*}$ for both channels, which are indicated by the red arrows, will be adequate for such selections.

\section{Expected rates and statistical precision}

We present the expected numbers of events for exclusive production of $\mathrm{J} / \mathrm{\psi}$ or $\rho^{0}$ on protons and gold/lead ions in the collisions of $10 \mathrm{GeV}$ electrons and 100 $\mathrm{GeV} /$ mucleon hadron beams. We assume the instantenous luminosities equal to the minimal expected values for eRHIC given in Ref. 1 . We also assume the integrated luminosities per year $\int \mathcal{L}$ equal to $330 \mathrm{pb}^{-1}$ for $e p$ collisions and $10 \mathrm{pb}^{-1}$ for $e \mathrm{Au}$ collisions. Each number correspond to 2.5 weeks of effective data taking.

Using parametrisations of the cross sections for $J / \psi$ and $\rho^{0}$ exclusive production 
at HERA and extrapolating them down in energy we expect for the kinematic range of the proposed detector, $1<Q^{2}<50 \mathrm{GeV}^{2}$ and $10<W<60 \mathrm{GeV}$, the following cross sections: $\sigma_{e p \rightarrow e J / \psi p}=0.2 \mathrm{nb}$ and $\sigma_{e p \rightarrow e \rho^{0} p}=5.2 \mathrm{nb}$. For the estimates of the cross sections for heavy nucleus we have made rather extreme assumptions: no nuclear absorption for $J / \psi$ production and strong absorption for $\rho^{0}$ similar to that observed for elastic scattering of pions on nuclei. The nuclear absorption of $\rho^{0}$ was described in the impuls approximation by introducing the effective number of interacting nucleons $A_{\text {eff }}=A^{0.75}$, the same for coherent and incoherent parts of the cross section.

Taking into account the acceptance of the extended central detector discussed earlier, we expect the total number of.DIS exclusive $J / \psi$ events to be 60000 for the proton beam and almost $2 \mathrm{mln}$ for heavy ion beam, and for $\rho^{0}$ channel $\sim 2$ mln events and $\sim 4$ mln events correspondingly (see Fig 21 ).

The statistical precision of the single differential cross sections as a function of $x$ or $Q^{2}$ is presented in Figs 22 and 23. By splitting the data in 6 bins indicated in the figures one could get the precision which in general is better than $1 \%$ for proton bearn and better than $0.5 \%$ for a heavy ion beam. Only at $Q^{2}>15 \mathrm{GeV}^{2}$ the statistical accuracy increases to few percent.

\section{Conslusions and plans}

High-accuracy tracking detectors covering the range $5<\theta<175^{\circ}$, e.g. drift chambers and silicon detectors, are essential for measuring DIS exclusive $J / \psi$ and $\rho^{0}$ production at eRHIC. The mid-forward and mid-rear ranges, $5<\theta<20^{\circ}$ and $160<\theta<175^{\circ}$, correlate with the lowest $x$ range.

High statistical accuracy, $0.1 \%$ to $2 \%$ for about 2.5 weeks of data taking, may be obtained with the instantenous luminosities equal to the minimum expected values.

Good resolutions in $Q^{2}, x, t$ and $\cos \theta^{*}$ are feasible.

We plan to complement the presented analysis by the following elements:

- Feasibility studies for the leading particle spectrometer,

- Detector requirements for measuring DVCS,

- Detector requirements for measuring exclusive $J / \psi$ photoproduction and production at very small $Q^{2}$.

\section{References}

[1] I. Ben-Zvi, J. Kewisch, J. Murphy, S. Peggs, "Accelerator Physics issues in eRHIC", C-A/AP/14, April 3, 2000. 